\title{
Quaternionic Analysis, Representation Theory and Physics II
}

\author{
Igor Frenkel and Matvei Libine
}

November 15, 2019

\begin{abstract}
We develop further quaternionic analysis introducing left and right doubly regular functions. We derive Cauchy-Fueter type formulas for these doubly regular functions that can be regarded as another counterpart of Cauchy's integral formula for the second order pole, in addition to the one studied in the first paper with the same title. We also realize the doubly regular functions as a subspace of the quaternionic-valued functions satisfying a Euclidean version of Maxwell's equations for the electromagnetic field.

Then we return to the study of the original quaternionic analogue of Cauchy's second order pole formula and its relation to the polarization of vacuum. We find the decomposition of the space of quaternionic-valued functions into irreducible components that include the spaces of doubly left and right regular functions. Using this decomposition, we show that a regularization of the vacuum polarization diagram is achieved by subtracting the component corresponding to the one-dimensional subrepresentation of the conformal group. After the regularization, the vacuum polarization diagram is identified with a certain second order differential operator which yields a quaternionic version of Maxwell equations.

Next, we introduce two types of quaternionic algebras consisting of spaces of scalarvalued and quaternionic-valued functions. We emphasize that these algebra structures are invariant under the action of the conformal Lie algebra. This is done using techniques that appear in the study of the vacuum polarization diagram. These algebras are not associative, but we can define an infinite family of $n$-multiplications, and we conjecture that they have the structures of weak cyclic $A_{\infty}$-algebras. We also conjecture the relation between the multiplication operations of the scalar and non-scalar quaternionic algebras with the $n$ photon Feynman diagrams in the scalar and ordinary conformal QED.

We conclude the article with a discussion of relations between quaternionic analysis, representation theory of the conformal group, massless quantum electrodynamics and perspectives of further development of these subjects.
\end{abstract}

\section{Introduction}

The starting point in the development of quaternionic analysis by Fueter and others was an exact analogue of Cauchy's integral formula for complex holomorphic functions

$$
f(w)=\frac{1}{2 \pi i} \oint \frac{f(z)}{z-w} d z
$$

involving the first order pole. This counterpart of (11) is usually referred to as the Cauchy-Fueter formulas for the quaternionic analogues of holomorphic functions known as left and right regular functions. Thus there are two versions:

$$
\begin{aligned}
& f(W)=\frac{1}{2 \pi^{2}} \int_{S} k(Z-W) \cdot D z \cdot f(Z), \\
& g(W)=\frac{1}{2 \pi^{2}} \int_{S} g(Z) \cdot D z \cdot k(Z-W),
\end{aligned}
$$


where

$$
k(Z-W)=\frac{(Z-W)^{-1}}{\operatorname{det}(Z-W)}
$$

$D z$ is a certain quaternionic valued 3 -from, the contour of integration $S$ is homotopic to a 3sphere $S^{3}$ around $W$ in $\mathbb{H}, f: \mathbb{H} \rightarrow \mathbb{S}$ is left regular, and $g: \mathbb{H} \rightarrow \mathbb{S}^{\prime}$ is right regular. Here, $\mathbb{S}$ and $\mathbb{S}^{\prime}$ are two dimensional left and right modules over $\mathbb{H}$. (Usually people consider $\mathbb{H}$-valued functions.) The quaternionic conformal group $S L(2, \mathbb{H})$ and its Lie algebra $\mathfrak{s l}(2, \mathbb{H})$ have natural actions on the spaces of left and right regular functions, which are analogous to the actions of the (global) conformal group $S L(2, \mathbb{C})$ and its Lie algebra $\mathfrak{s l}(2, \mathbb{C})$ on the space of holomorphic functions. In spite of this indisputable parallel between complex and quaternionic analysis, further attempts to extend the analogy between the two theories have been met with substantial difficulties. In particular, neither left nor right regular functions form a ring and, therefore, cannot be regarded as a full counterpart of the ring of holomorphic functions. Additionally, generalizations of the Cauchy-Fueter formulas (2)-(3) to higher order poles are not at all straightforward.

In our first paper with the same title [FL1] we proposed to approach quaternionic analysis from the point of view of representation theory of the conformal group $S L(2, \mathbb{H})$ and its Lie algebra $\mathfrak{s l}(2, \mathbb{H})$. In particular, we explored the parallel between quaternionic and complex analysis from this representation theoretic point of view. This approach allowed us to discover a quaternionic counterpart of Cauchy's integral formula for the second order pole

$$
\frac{d f}{d w}(w)=\frac{1}{2 \pi i} \oint \frac{f(z) d z}{(z-w)^{2}}
$$

by interpreting the square of the Cauchy-Fueter kernel (44) as a kernel of an intertwining operator for $\mathfrak{s l}(2, \mathbb{H})$. As explained in Introduction of [FL1], the derivative operator $\frac{d}{d z}$ can be interpreted as an intertwining operator between certain representations of $S L(2, \mathbb{C})$. We show in [FL1] that the quaternionic counterpart of (5) dictated by representation theory of the quaternionic conformal group has the form

$$
(\mathrm{Mx} F)(W)=\frac{12 i}{\pi^{3}} \int_{Z \in U(2)} k(Z-W) \cdot F(Z) \cdot k(Z-W) d V
$$

where the operator $\frac{d}{d z}$ in Cauchy's formula (5) is replaced by a certain second order differential operator that we call "Maxwell operator"

$$
\mathrm{Mx} F=\vec{\nabla} F \overleftarrow{\nabla}-\square F^{+}
$$

The operator $\mathrm{Mx}$ is an intertwining operator between certain actions of $\mathfrak{s l}(2, \mathbb{H})$ on the space of quaternionic valued polynomials (or its analytic completion).

In complex analysis, Cauchy's formulas for the first and second order poles (11), (5D) admit immediate generalizations to holomorphic functions on the punctured complex plane $\mathbb{C}^{\times}=$ $\mathbb{C} \backslash\{0\}$ by choosing the contour of integration to be the difference of loops around zero and infinity. In quaternionic analysis, there is a similar generalization of the Cauchy-Fueter formulas (2) -(3) to regular functions on $\mathbb{H}^{\times}=\mathbb{H} \backslash\{0\}$ by choosing the contour of integration to be the difference of two 3-cycles around zero and infinity (as well as more general domains). However, a generalization of the quaternionic analogue of the second order pole formula (5) to functions on $\mathbb{H}^{\times}$presents substantial difficulties and is directly related to the divergence of the Feynman diagram for vacuum polarization, as was indicated in [FL1]. In the present paper we resolve this problem similarly to our derivation of the quaternionic second order pole formula for the scalar valued functions in $\left[\right.$ FL3]. Recall that $\mathbb{D}^{+}$and $\mathbb{D}^{-}$are certain open domains in $\mathbb{H} \otimes \mathbb{C}$, both having $U(2)$ as Shilov boundary. The idea is to separate the singularities in the second order 
pole by considering maps $J^{\varepsilon_{1} \varepsilon_{2}}$, where $\varepsilon_{1}, \varepsilon_{2}= \pm$, from a space of quaternionic valued functions $\mathcal{W}^{\prime}$ to (a completion of) the tensor product of left and right regular functions

$$
\begin{aligned}
\left(J^{\varepsilon_{1} \varepsilon_{2}} F\right)\left(Z_{1}, Z_{2}\right)= & \frac{12 i}{\pi^{3}} \int_{W \in U(2)} k\left(W-Z_{1}\right) \cdot F(W) \cdot k\left(W-Z_{2}\right) d V \\
& \text { with } \quad Z_{1} \in \mathbb{D}^{\varepsilon_{1}}, \quad Z_{2} \in \mathbb{D}^{\varepsilon_{2}},
\end{aligned}
$$

and then taking the limits as $Z_{1}, Z_{2}$ approach the common boundary $U(2)$ of $\mathbb{D}^{ \pm}$. While the maps $J^{++}$and $J^{--}$are well defined on the diagonal $Z_{1}=Z_{2}$ (as in [FL1]), the maps $J^{+-}$and $J^{-+}$have singularities which cancel each other in the sum (as in [FL3]). Setting $Z_{1}=Z_{2}$ in the total map

$$
J=J^{++}+J^{--}-J^{+-}-J^{-+}
$$

yields an extension of our second order pole formula to functions on $\mathbb{H}^{\times}$

$$
(J F)(Z, Z)=(\mathrm{Mx} F)(Z) .
$$

The appearance of singularity in $J^{+-}, J^{-+}$is related to the presence of the one-dimensional representation in the subquotient of $\mathcal{W}^{\prime}$. For this reason, the generalization of our second order pole formula from [FL1] to the "quaternionic Laurent polynomials" - i.e. polynomial functions defined on $\mathbb{H}^{\times}$- requires a detailed study of the $\mathfrak{s l}(2, \mathbb{H})$-module structures of the spaces $\mathcal{W}^{\prime}$ and $\mathcal{W}$ of quaternionic valued functions (now defined on $\mathbb{H}^{\times}$) and the homomorphism $\mathrm{Mx}: \mathcal{W}^{\prime} \rightarrow \mathcal{W}$. It turns out that each of these modules contains 13 composition factors, and they can be studied using a larger complex originally considered as equation (57) in [FL1]:

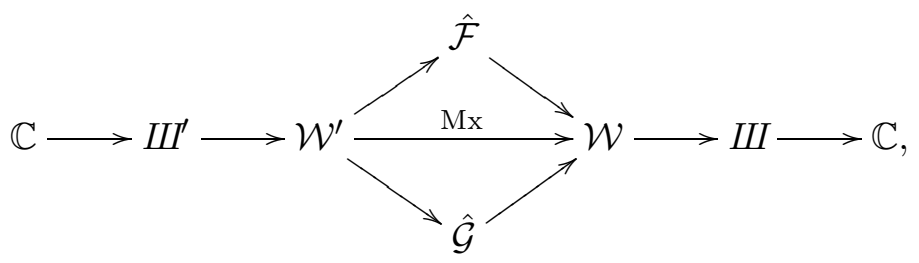

where $H^{\prime}$ is the space of scalar valued functions on $\mathbb{H}^{\times}$and $I I$ is its dual space. We show that both $I I$ and $I^{\prime}$ have six irreducible components, five of which reappear in $\mathcal{W}$ and $\mathcal{W}^{\prime}$, and the trivial one-dimensional subrepresentation $\mathbb{C}$ of $\amalg^{\prime}$ is annihilated by $\nabla^{+}$.

The spaces $\hat{\mathcal{F}}$ and $\hat{\mathcal{G}}$ are

$$
\hat{\mathcal{F}}=\left\{f: \mathbb{H}^{\times} \rightarrow \mathbb{S} \otimes \mathbb{S}\right\} \quad \text { and } \quad \hat{\mathcal{G}}=\left\{g: \mathbb{H}^{\times} \rightarrow \mathbb{S}^{\prime} \otimes \mathbb{S}^{\prime}\right\} .
$$

They contain subspaces $\mathcal{F}$ and $\mathcal{G}$ of "doubly left and right regular functions" respectively that share many similarities with the usual left and right regular functions. They are preserved by the actions of the conformal group and can be defined as kernels of certain linear differential operators. Besides, they also satisfy a quaternionic analogue of Cauchy's integral formula for the second order pole (5) as follows:

$$
\begin{aligned}
& \nabla(W \cdot f)(W)=\frac{1}{\pi^{2}} \int_{S} k_{1}(Z-W) \cdot D z \cdot Z \cdot f(Z) \\
& (g(W) \cdot W) \overleftarrow{\nabla}=\frac{1}{\pi^{2}} \int_{S} g(Z) \cdot Z \cdot D z \cdot k_{1}(Z-W)
\end{aligned}
$$

where

$$
k_{1}(Z-W)=\frac{1}{2} \nabla k(Z-W)
$$


$f: \mathbb{H} \rightarrow \mathbb{S} \otimes \mathbb{S}$ is a doubly left regular function and $g: \mathbb{H} \rightarrow \mathbb{S}^{\prime} \otimes \mathbb{S}^{\prime}$ is a doubly right regular function. Like the Cauchy-Fueter formulas (2)-(3), formulas (9)-(10) extend to functions on $\mathbb{H}^{\times}$ and more general domains.

We emphasize that in quaternionic analysis there are two analogues of Cauchy's integral formula for the second order pole (5). On the one hand, the kernel $(z-w)^{-2}$ in (5) can be viewed as a square of $(z-w)^{-1}$ with the quaternionic counterpart having form (6). On the other hand, the kernel $(z-w)^{-2}$ can also be regarded as a derivative of $(z-w)^{-1}$, in which case the quaternionic counterpart is given by (9)-(10). Although the two quaternionic analogues (6) and (9)-(10) of Cauchy's integral formula for the second order pole appear to be quite different, they turn out to be complementary to each other. In fact, one can identify the doubly left and right regular functions with self-dual and anti-self-dual solutions of the Maxwell equations (in Euclidean signature). Moreover, (9)-(10) can be combined into a single formula valid for any quaternionic functions $A(Z)$ annihilated by the Maxwell operator:

$$
\operatorname{Mx} A(Z)=0, \quad A: \mathbb{H}^{\times} \rightarrow \mathbb{H} .
$$

Comparing (6) and (11) clearly demonstrates the complementary nature of the two quaternionic analogues (6) and (99)-(10) of the second order pole formula and that the Maxwell equations play a key role in both versions!

Our study of the quaternionic complex and the decomposition of the representations involved in (8) uses extensively the basis of $K$-types of $(\mathfrak{g}, K)$-modules with $\mathfrak{g}=\mathfrak{g l}(2, \mathbb{H}) \otimes \mathbb{C} \simeq \mathfrak{g l}(4, \mathbb{C})$ and $K=U(2) \times U(2)$. Therefore, the algebra generated by the matrix coefficients of $G L(2, \mathbb{C})$ in finite dimensional representations $\left(t_{n \underline{m}}^{l}(Z)\right.$ 's and $N(Z)^{k}$ 's) plays a key role in our approach. This algebra can be viewed as a counterpart of the algebra of Laurent polynomials in complex analysis, which is the algebra of the matrix coefficients of $G L(1, \mathbb{C})$. The algebra of matrix coefficients of $G L(2, \mathbb{C})$ technically is more complicated than the familiar algebra of Laurent polynomials, but conceptually various results and formulas in many aspects are similar. The bases of $K$-types that we are using have certain advantages over the equivalent picture in the Minkowski space, where simpler continuous bases are natural (see e.g. [FL3], Section 8, for the relation between the two types of bases). In particular, the $K$-bases allow us to conveniently isolate the one-dimensional irreducible component in representations $\mathcal{W}$ and $\mathcal{W}^{\prime}$, which plays crucial role in regularization of the vacuum polarization. This component is less transparent in the Minkowski picture and is hidden in the traditional physics approach to this regularization. The $K$-type approach is extensively used in analysis of various representations of real semisimple groups, see e.g. Le and especially Section 8 dedicated to the $K$-types of representations of $S U(2,2)$.

We saw in our first paper [FL1] that in order to introduce unitary structures on the spaces of harmonic as well as (left and right) regular functions, one must replace the quaternionic conformal group $S L(2, \mathbb{H})$ with $S U(2,2)$, which is another real form of $S L(4, \mathbb{C})$. The group $S U(2,2)$ in turn can be identified with the conformal group of the Minkowski space $\mathbb{M}$. Similarly, the unitarity of the spaces of doubly (left and right) regular functions and, equivalently, the space of solutions of the Maxwell equations (11) modulo the image of $W^{\prime}$ require the same Minkowski space $\mathbb{M}$.

Moreover, the quaternionic complex (8) in Minkowski space realization can be identified with the complex of differential forms on the Minkowski space with the zero light cone removed. The program of study of vector bundles on the covering space of the compactified Minkowski space and representations of the conformal group in the spaces of sections was suggested by I. Segal as a mathematical approach to studying of four-dimensional field theory. In particular, the irreducible components of the complex of differential forms were identified by his student S. Paneitz $[\mathrm{P}]$. Thus quaternionic analysis and representation theory of the conformal group 
associated to Minkowski space are deeply intertwined and mutually beneficial. Another example of this link is provided by the realization of irreducible representations of the most degenerate series (depending on $n \in \mathbb{Z}$ ) as solutions of certain differential operators on $\mathbb{M}$ [JV1]. For $n= \pm 1$, these spaces are exactly the spaces of left/right regular functions. And for $n= \pm 2$, they can be identified with the doubly left/right regular functions. One can also define $n$-regular functions for any $n \in \mathbb{N}$, then the Cauchy-Fueter type integral formulas for these functions can be interpreted as a quaternionic analogue of Cauchy's integral formula for the $n$-th order pole, where the Cauchy kernel is treated as the $(n-1)$-st derivative of $(z-w)^{-1}$.

The quaternionic second order pole formulas described above show that the analogy with the complex case is not straightforward. So, it is not surprising that the quaternionic counterpart of the algebra of complex holomorphic functions is far from obvious. In this paper we suggest a certain candidate for a quaternionic algebra, again, based on representation theory of the conformal group. We already noted that tensor products of representations of the most degenerate series of $S U(2,2)$ (and its Lie algebra) depending on $n \in \mathbb{Z}$ do not contain representations of the same class. For $n= \pm 1$, these representations are exactly the spaces of left/right regular functions. And for $n= \pm 2$, these representations can be identified with the doubly left/right regular functions. Therefore, one cannot expect a group-invariant algebra structure on these function spaces. Thus we have to consider the class of representations that comes next after the most degenerate series of $S U(2,2)$ - the middle series. Such representations appear in the complex of quaternionic spaces (8), and one can consider other similar representations, for example, the space of $\mathbb{C}$-valued functions $\mathscr{W}$ studied in [FL3]. Clearly, the space $W^{\prime}$ provides a trivial example of a quaternionic algebra of $\mathbb{C}$-valued functions with pointwise multiplication. On the other hand, the best candidate for a quaternionic algebra of quaternionic-valued functions appears to be a closely related space $\mathcal{W}^{\prime}$. In order to understand the quaternionic algebra structure, we start with the algebra of scalar-valued quaternionic functions $\mathscr{W}$. It is similar to, but in certain ways simpler than $\mathcal{W}^{\prime}$. In both cases we first embed our spaces into larger algebras

$$
I: \mathscr{K} \rightarrow \text { completion of } \mathcal{H} \otimes \mathcal{H}, \quad J: \mathcal{W}^{\prime} \rightarrow \text { completion of } \mathcal{V} \otimes \mathcal{V}^{\prime},
$$

where $I$ is described in [FL3] and $J$ appears in our study of vacuum polarization in this paper. Note that $\mathcal{H}$ is the space of harmonic functions on $\mathbb{H}^{\times}$and $\mathcal{V}, \mathcal{V}^{\prime}$ are the spaces of left and right regular functions on $\mathbb{H}^{\times}$. Both $\mathcal{H}$ and $\mathcal{V}, \mathcal{V}^{\prime}$ are representations of the most degenerate series corresponding to $n=0$ and $n= \pm 1$ respectively. The multiplication in the larger algebra is defined using the invariant pairings on $\mathcal{H}$ and between $\mathcal{V}$ and $\mathcal{V}^{\prime}$. Thus, to finish our construction of quaternionic multiplication operation, we need to find appropriate inverses of the maps $I$ and $J$. This can be done in two ways by considering certain subtle limits and, therefore, one can define a one-parameter family of invariant multiplications by taking linear combinations of those limits. The case of $\mathcal{W}^{\prime}$ differs from $\mathscr{W}$ in that ker $J$ is non-trivial and, therefore, we actually define multiplication on $\mathcal{W}^{\prime} /$ ker $J$. An additional subtlety of the $\mathcal{W}^{\prime}$ case is that in the construction of the inverse of $J$ we lose the one-dimensional irreducible component of the representation $\mathcal{W}^{\prime} /$ ker $J$; this is the component containing the identity element. Thus we can only define a one-parameter family of multiplication-like operations

$$
\left(\mathcal{W}^{\prime} / \operatorname{ker} J\right) \otimes\left(\mathcal{W}^{\prime} / \operatorname{ker} J\right) \rightarrow\left(\mathcal{W}^{\prime} / \operatorname{ker} J\right) / \mathbb{C} \simeq \mathcal{W}^{\prime} / \operatorname{ker} \mathrm{Mx}
$$

that are invariant under the action of the conformal group. These maps can be lifted to genuine multiplication operations

$$
\left(\mathcal{W}^{\prime} / \operatorname{ker} J\right) \otimes\left(\mathcal{W}^{\prime} / \operatorname{ker} J\right) \rightarrow \mathcal{W}^{\prime} / \operatorname{ker} J
$$

similarly to the procedure of adjoining of unit to algebras without units. It is an interesting problem to find a way to define the multiplication on $\mathcal{W}^{\prime} /$ ker $J$ directly without the procedure of adjoining the unit. Both quaternionic algebras $\mathcal{W}$ and $\mathcal{W}^{\prime} /$ ker $J$ defined in this paper 


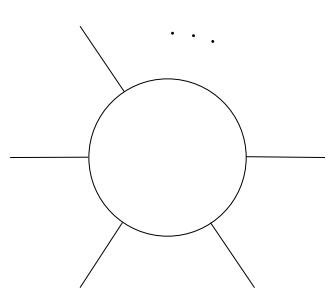

(a) Scalar case

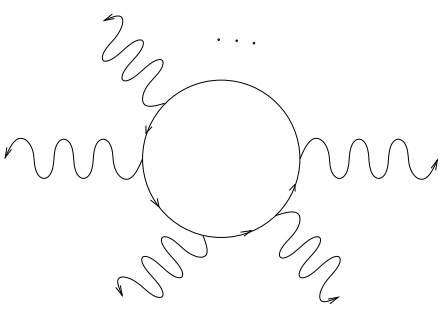

(b) Spinor case

Figure 1: n-photon Feynman diagrams.

are not associative. However, our construction allows immediate generalizations to invariant $n$-multiplications (multiplications of $n$ factors) with $n=1$ corresponding to the identity operator and $n=2$ to the multiplication described above. We conjecture that the resulting $n$-multiplications satisfy the quadratic relations of weak cyclic $A_{\infty}$-algebras. Thus the quaternionic analogue of the algebra of complex holomorphic functions might have a much richer structure than its classical counterpart! Another interesting question is how to characterize the $n$-multiplications as intertwining operators for the conformal group.

We see repeatedly in [FL1 and in this paper that the Minkowski space reformulation of various structures of quaternionic analysis leads to profound relations with different structures of four-dimensional conformal field theory, particularly with the conformal QED. One can ask about the physical meaning of the two quaternionic algebras $\mathcal{W}$ and $\mathcal{W}^{\prime} /$ ker $J$, including their $n$-multiplications and possible relations between them. Our second conjecture is that they are related to the $n$-photon diagrams in the scalar and non-scalar conformal QED (Figure 1). We discuss this conjecture in more detail in Subsection 7.5 at the end of the paper. For now, we only mention the relation between our two conjectures. Namely, it was discovered relatively recently (more than fifty years after creation of modern QED) that $n$-photon diagrams (as well as $n$ gluon diagrams) satisfy certain quadratic relations, known in physics literature as the BCFW relations (see [BCFW], [BBBV] and references therein).

These relations provide strong evidence in support of and a link between our two conjectures. In particular, if the second conjecture is correct, then the BCFW relations yield the associativitytype relations for the quaternionic algebras giving them the structures of weak cyclic $A_{\infty}$ algebras, thus making the first conjecture valid as well. This way studying quaternionic algebras and relating structures will produce further connections between quaternionic analysis and fourdimensional quantum field theory that we predicted in the first paper with the same title [FL1]. We expect that these connections will be beneficial for both disciplines: quaternionic analysis will be enriched by many beautiful structures and quantum field theory will find its purely mathematical formulation.

For technical reasons the paper is organized differently from the order of this discussion. In Section 2 we define and study left and right doubly regular functions. This is done in complete parallel with the theory of regular functions reviewed in [FL1]: we prove analogues of the Cauchy-Fueter formulas, construct action of the conformal group, decompose the CauchyFueter kernel for these functions and study the invariant bilinear form. In the last subsection we generalize the notion of doubly regular functions to $n$-regular functions. The Cauchy-Fueter type formulas for these functions are proved in a separate paper [FL4]. In Section 3 we describe the quaternionic chain complex (8), which plays a central role in this paper. Then we decompose the representations $W$ and $W^{\prime}$ into irreducible components. In Section 4 we proceed to the study of representations $\mathcal{W}$ and $\mathcal{W}^{\prime}$. First, we analyze the kernel of $\mathrm{Mx}$ in $\mathcal{W}^{\prime}$ which contains the image 
of $H^{\prime}$ and the irreducible components isomorphic to doubly left and right regular functions. We carefully identify the $K$-types and explicit forms of various intertwining functors related to the quaternionic chain complex (8). Some of the intertwining functors are expressed by quaternionic analogues of Cauchy's integral formula (5). In Section 5 we complete the decomposition of representations $\mathcal{W}$ and $\mathcal{W}^{\prime}$. Besides the five irreducible components coming from $\amalg$ and $W^{\prime}$ and four irreducible components of doubly regular functions, we identify four additional irreducible components, including the trivial one-dimensional representation. These four components are crucial to understanding of polarization of vacuum and definition of quaternionic algebra that are subject of Section [6. In Section [6 we generalize our result from [FL1] and extend the quaternionic analogue of Cauchy's integral formula for the second order pole from $\mathcal{W}^{\prime+}$ to $\mathcal{W}^{\prime}$. Our main technical tool is a certain operator

$$
J: \mathcal{W}^{\prime} \rightarrow \text { completion of } \mathcal{V} \otimes \mathcal{V}^{\prime}
$$

The quotient space $\mathcal{W}^{\prime}$ / ker $J$ has four irreducible components, including the trivial one-dimensional subrepresentation. In the subsequent section, $\mathcal{W}^{\prime} / \operatorname{ker} J$ will be equipped with an algebra structure. In Section 7 we first construct a scalar quaternionic algebra using previous results from FL3. Then we proceed in a similar fashion to our main goal of constructing the quaternionic algebra structure on the space $\mathcal{W}^{\prime} /$ ker $J$. The latter version is in many aspects similar to its scalar counterpart, but has a richer structure. In Subsection 7.5 we discuss relations between quaternionic algebras and Feynman diagrams of massless QED as well as future problems and perspectives of our direction of quaternionic analysis. In Section 8 we provide some comments about our earlier papers [FL1, FL3 that are relevant to the present article.

Since this paper is a continuation of [FL1, FL3], we follow the same notations and instead of introducing those notations again we direct the reader to Section 2 of [FL3].

\section{Doubly Regular Functions}

\subsection{Definitions}

We continue to use notations established in [FL1]. In particular, $e_{0}, e_{1}, e_{2}, e_{3}$ denote the units of the classical quaternions $\mathbb{H}$ corresponding to the more familiar $1, i, j, k$ (we reserve the symbol $i$ for $\sqrt{-1} \in \mathbb{C}$ ). Thus $\mathbb{H}$ is an algebra over $\mathbb{R}$ generated by $e_{0}, e_{1}, e_{2}, e_{3}$, and the multiplicative structure is determined by the rules

$$
e_{0} e_{i}=e_{i} e_{0}=e_{i}, \quad\left(e_{i}\right)^{2}=e_{1} e_{2} e_{3}=-e_{0}, \quad e_{i} e_{j}=-e_{i} e_{j}, \quad 1 \leq i<j \leq 3,
$$

and the fact that $\mathbb{H}$ is a division ring. Next we consider the algebra of complexified quaternions (also known as biquaternions) $\mathbb{H}_{\mathbb{C}}=\mathbb{C} \otimes_{\mathbb{R}} \mathbb{H}$ and write elements of $\mathbb{H}_{\mathbb{C}}$ as

$$
Z=z^{0} e_{0}+z^{1} e_{1}+z^{2} e_{2}+z^{3} e_{3}, \quad z^{0}, z^{1}, z^{2}, z^{3} \in \mathbb{C},
$$

so that $Z \in \mathbb{H}$ if and only if $z^{0}, z^{1}, z^{2}, z^{3} \in \mathbb{R}$ :

$$
\mathbb{H}=\left\{X=x^{0} e_{0}+x^{1} e_{1}+x^{2} e_{2}+x^{3} e_{3} ; x^{0}, x^{1}, x^{2}, x^{3} \in \mathbb{R}\right\}
$$

Recall that we denote by $\mathbb{S}$ (respectively $\mathbb{S}^{\prime}$ ) the irreducible 2-dimensional left (respectively right)

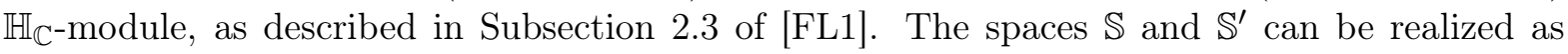
respectively columns and rows of complex numbers. Then

$$
\mathbb{S} \otimes \mathbb{S}^{\prime} \simeq \mathbb{H}_{\mathbb{C}}
$$


Note that $\mathbb{S} \otimes \mathbb{S}$ and $\mathbb{S}^{\prime} \otimes \mathbb{S}^{\prime}$ are respectively left and right modules over $\mathbb{H}_{\mathbb{C}} \otimes \mathbb{H}_{\mathbb{C}}$.

We introduce four first order differential operators

$$
\begin{aligned}
\nabla^{+} \otimes 1 & =\left(e_{0} \otimes 1\right) \frac{\partial}{\partial x^{0}}+\left(e_{1} \otimes 1\right) \frac{\partial}{\partial x^{1}}+\left(e_{2} \otimes 1\right) \frac{\partial}{\partial x^{2}}+\left(e_{3} \otimes 1\right) \frac{\partial}{\partial x^{3}}, \\
1 \otimes \nabla^{+} & =\left(1 \otimes e_{0}\right) \frac{\partial}{\partial x^{0}}+\left(1 \otimes e_{1}\right) \frac{\partial}{\partial x^{1}}+\left(1 \otimes e_{2}\right) \frac{\partial}{\partial x^{2}}+\left(1 \otimes e_{3}\right) \frac{\partial}{\partial x^{3}}, \\
\nabla \otimes 1 & =\left(e_{0} \otimes 1\right) \frac{\partial}{\partial x^{0}}-\left(e_{1} \otimes 1\right) \frac{\partial}{\partial x^{1}}-\left(e_{2} \otimes 1\right) \frac{\partial}{\partial x^{2}}-\left(e_{3} \otimes 1\right) \frac{\partial}{\partial x^{3}}, \\
1 \otimes \nabla & =\left(1 \otimes e_{0}\right) \frac{\partial}{\partial x^{0}}-\left(1 \otimes e_{1}\right) \frac{\partial}{\partial x^{1}}-\left(1 \otimes e_{2}\right) \frac{\partial}{\partial x^{2}}-\left(1 \otimes e_{3}\right) \frac{\partial}{\partial x^{3}},
\end{aligned}
$$

which can be applied to functions with values in $\mathbb{S} \otimes \mathbb{S}$ or $\mathbb{S}^{\prime} \otimes \mathbb{S}^{\prime}$ as follows. If $U$ is an open subset of $\mathbb{H}$ or $\mathbb{H}_{\mathbb{C}}$ and $f: U \rightarrow \mathbb{S} \otimes \mathbb{S}$ is a differentiable function, then these operators can be applied to $f$ on the left. For example,

$$
\left(\nabla^{+} \otimes 1\right) f=\left(e_{0} \otimes 1\right) \frac{\partial f}{\partial x^{0}}+\left(e_{1} \otimes 1\right) \frac{\partial f}{\partial x^{1}}+\left(e_{2} \otimes 1\right) \frac{\partial f}{\partial x^{2}}+\left(e_{3} \otimes 1\right) \frac{\partial f}{\partial x^{3}} .
$$

Similarly, these operators can be applied on the right to differentiable functions $g: U \rightarrow \mathbb{S}^{\prime} \otimes \mathbb{S}^{\prime}$; we often indicate this with an arrow above the operator. For example,

$$
g\left(\overleftarrow{\nabla^{+} \otimes 1}\right)=\frac{\partial g}{\partial x^{0}}\left(e_{0} \otimes 1\right)+\frac{\partial g}{\partial x^{1}}\left(e_{1} \otimes 1\right)+\frac{\partial g}{\partial x^{2}}\left(e_{2} \otimes 1\right)+\frac{\partial g}{\partial x^{3}}\left(e_{3} \otimes 1\right)
$$

The tensor product $\mathbb{S} \otimes \mathbb{S}$ decomposes into a direct sum of its symmetric part $\mathbb{S} \odot \mathbb{S}$ and antisymmetric part $\mathbb{S} \wedge \mathbb{S}$ :

$$
\mathbb{S} \otimes \mathbb{S}=(\mathbb{S} \odot \mathbb{S}) \oplus(\mathbb{S} \wedge \mathbb{S})
$$

Similarly, $\mathbb{S}^{\prime} \otimes \mathbb{S}^{\prime}$ decomposes into a direct sum of its symmetric and antisymmetric parts:

$$
\mathbb{S}^{\prime} \otimes \mathbb{S}^{\prime}=\left(\mathbb{S}^{\prime} \odot \mathbb{S}^{\prime}\right) \oplus\left(\mathbb{S}^{\prime} \wedge \mathbb{S}^{\prime}\right)
$$

Definition 1. Let $U$ be an open subset of $\mathbb{H}$. A $\mathcal{C}^{1}$-function $f: U \rightarrow \mathbb{S} \odot \mathbb{S}$ is doubly left regular if it satisfies

$$
\left(\nabla^{+} \otimes 1\right) f=0 \quad \text { and } \quad\left(1 \otimes \nabla^{+}\right) f=0
$$

for all points in $U$. Similarly, a $\mathcal{C}^{1}$-function $g: U \rightarrow \mathbb{S}^{\prime} \odot \mathbb{S}^{\prime}$ is doubly right regular if

$$
g\left(\overleftarrow{\nabla^{+} \otimes 1}\right)=0 \quad \text { and } \quad g\left(\overleftarrow{1 \otimes \nabla^{+}}\right)=0
$$

for all points in $U$.

Since

$$
\begin{gathered}
(\nabla \otimes 1)\left(\nabla^{+} \otimes 1\right)=\left(\nabla^{+} \otimes 1\right)(\nabla \otimes 1)=(1 \otimes \nabla)\left(1 \otimes \nabla^{+}\right)=\left(1 \otimes \nabla^{+}\right)(1 \otimes \nabla)=\square, \\
\square=\frac{\partial^{2}}{\left(\partial x^{0}\right)^{2}}+\frac{\partial^{2}}{\left(\partial x^{1}\right)^{2}}+\frac{\partial^{2}}{\left(\partial x^{2}\right)^{2}}+\frac{\partial^{2}}{\left(\partial x^{3}\right)^{2}},
\end{gathered}
$$

doubly left and right regular functions are harmonic.

One way to construct doubly left regular functions is to start with a harmonic function $\varphi: \mathbb{H} \rightarrow \mathbb{S} \odot \mathbb{S}$, then $(\nabla \otimes \nabla) \varphi$ is doubly left regular. Similarly, if $\varphi: \mathbb{H} \rightarrow \mathbb{S}^{\prime} \odot \mathbb{S}^{\prime}$ is harmonic, then $\varphi(\overleftarrow{\nabla \otimes \nabla})$ is doubly right regular.

We also can talk about doubly regular functions defined on open subsets of $\mathbb{H}_{\mathbb{C}}$. In this case we require such functions to be holomorphic. 
Definition 2. Let $U$ be an open subset of $\mathbb{H}_{\mathbb{C}}$. A holomorphic function $f: U \rightarrow \mathbb{S} \odot \mathbb{S}$ is doubly left regular if it satisfies $\left(\nabla^{+} \otimes 1\right) f=0$ and $\left(1 \otimes \nabla^{+}\right) f=0$ for all points in $U$.

Similarly, a holomorphic function $g: U \rightarrow \mathbb{S}^{\prime} \odot \mathbb{S}^{\prime}$ is doubly right regular if $g\left(\overleftarrow{\nabla^{+} \otimes 1}\right)=0$ and $g\left(\overleftarrow{1 \otimes \nabla^{+}}\right)=0$ for all points in $U$.

Let $\mathcal{D} \mathcal{R}$ and $\mathcal{D} \mathcal{R}^{\prime}$ denote respectively the spaces of (holomorphic) doubly left and right regular functions on $\mathbb{H}_{\mathbb{C}}$, possibly with singularities.

Theorem 3. 1. The space $\mathcal{D} \mathcal{R}$ of doubly left regular functions $\mathbb{H}_{\mathbb{C}} \rightarrow \mathbb{S} \odot \mathbb{S}$ (possibly with singularities) is invariant under the following action of $G L\left(2, \mathbb{H}_{\mathbb{C}}\right)$ :

$$
\begin{array}{r}
\pi_{d l}(h): f(Z) \mapsto\left(\pi_{d l}(h) f\right)(Z)=\frac{(c Z+d)^{-1} \otimes(c Z+d)^{-1}}{N(c Z+d)} \cdot f\left((a Z+b)(c Z+d)^{-1}\right), \\
h^{-1}=\left(\begin{array}{ll}
a & b \\
c & d
\end{array}\right) \in G L\left(2, \mathbb{H}_{\mathbb{C}}\right) .
\end{array}
$$

2. The space $\mathcal{D} \mathcal{R}^{\prime}$ of doubly right regular functions $\mathbb{H}_{\mathbb{C}} \rightarrow \mathbb{S}^{\prime} \odot \mathbb{S}^{\prime}$ (possibly with singularities) is invariant under the following action of $G L\left(2, \mathbb{H}_{\mathbb{C}}\right)$ :

$$
\begin{array}{r}
\pi_{d r}(h): g(Z) \mapsto\left(\pi_{d r}(h) g\right)(Z)=g\left(\left(a^{\prime}-Z c^{\prime}\right)^{-1}\left(-b^{\prime}+Z d^{\prime}\right)\right) \cdot \frac{\left(a^{\prime}-Z c^{\prime}\right)^{-1} \otimes\left(a^{\prime}-Z c^{\prime}\right)^{-1}}{N\left(a^{\prime}-Z c^{\prime}\right)}, \\
h=\left(\begin{array}{cc}
a^{\prime} & b^{\prime} \\
c^{\prime} & d^{\prime}
\end{array}\right) \in G L\left(2, \mathbb{H}_{\mathbb{C}}\right) . \quad(14)
\end{array}
$$

Proof. It is easy to see that the formulas describing the actions $\pi_{d l}$ and $\pi_{d r}$ also produce welldefined actions on the spaces of all functions on $\mathbb{H}_{\mathbb{C}}$ (possibly with singularities) with values in $\mathbb{S} \otimes \mathbb{S}$ and $\mathbb{S}^{\prime} \otimes \mathbb{S}^{\prime}$ respectively. These actions preserve the subspaces of functions with values in $\mathbb{S} \odot \mathbb{S}$ and $\mathbb{S}^{\prime} \odot \mathbb{S}^{\prime}$. Differentiating $\pi_{d l}$ and $\pi_{d r}$, we obtain actions of the Lie algebra $\mathfrak{g l}\left(2, \mathbb{H}_{\mathbb{C}}\right)$, which we still denote by $\pi_{d l}$ and $\pi_{d r}$ respectively. Using notations

$$
\partial=\left(\begin{array}{cc}
\partial_{11} & \partial_{21} \\
\partial_{12} & \partial_{22}
\end{array}\right)=\frac{1}{2} \nabla, \quad \partial^{+}=\left(\begin{array}{cc}
\partial_{22} & -\partial_{21} \\
-\partial_{12} & \partial_{11}
\end{array}\right)=\frac{1}{2} \nabla^{+}, \quad \partial_{i j}=\frac{\partial}{\partial z_{i j}},
$$

we can describe these actions of the Lie algebra.

Lemma 4. The Lie algebra action $\pi_{d l}$ of $\mathfrak{g l}\left(2, \mathbb{H}_{\mathbb{C}}\right)$ on $\mathcal{D} \mathcal{R}$ is given by

$$
\begin{aligned}
& \pi_{d l}\left(\begin{array}{cc}
A & 0 \\
0 & 0
\end{array}\right): f(Z) \mapsto-\operatorname{Tr}(A Z \partial) f, \\
& \pi_{d l}\left(\begin{array}{ll}
0 & B \\
0 & 0
\end{array}\right): f(Z) \mapsto-\operatorname{Tr}(B \partial) f, \\
& \pi_{d l}\left(\begin{array}{cc}
0 & 0 \\
C & 0
\end{array}\right): f(Z) \mapsto \operatorname{Tr}(Z C Z \partial+C Z) f+(C Z \otimes 1+1 \otimes C Z) f, \\
& \pi_{d l}\left(\begin{array}{ll}
0 & 0 \\
0 & D
\end{array}\right): f(Z) \mapsto \operatorname{Tr}(Z D \partial+D) f+(D \otimes 1+1 \otimes D) f .
\end{aligned}
$$

Similarly, the Lie algebra action $\pi_{d r}$ of $\mathfrak{g l}\left(2, \mathbb{H}_{\mathbb{C}}\right)$ on $\mathcal{D} \mathcal{R}^{\prime}$ is given by

$$
\begin{aligned}
& \pi_{d r}\left(\begin{array}{ll}
A & 0 \\
0 & 0
\end{array}\right): g(Z) \mapsto-\operatorname{Tr}(A Z \partial+A) g-g(A \otimes 1+1 \otimes A), \\
& \pi_{d r}\left(\begin{array}{ll}
0 & B \\
0 & 0
\end{array}\right): g(Z) \mapsto-\operatorname{Tr}(B \partial) g, \\
& \pi_{d r}\left(\begin{array}{ll}
0 & 0 \\
C & 0
\end{array}\right): g(Z) \mapsto \operatorname{Tr}(Z C Z \partial+Z C) g+g(Z C \otimes 1+1 \otimes Z C), \\
& \pi_{d r}\left(\begin{array}{ll}
0 & 0 \\
0 & D
\end{array}\right): g(Z) \mapsto \operatorname{Tr}(Z D \partial) g .
\end{aligned}
$$

Proof. These formulas are obtained by differentiating (13) and (14). 
We return to the proof of Theorem 3 . Since the Lie group $G L\left(2, \mathbb{H}_{\mathbb{C}}\right) \simeq G L(4, \mathbb{C})$ is connected, it is sufficient to show that, if $f \in \mathcal{D} \mathcal{R}, g \in \mathcal{D} \mathcal{R}^{\prime}$ and $\left(\begin{array}{ll}A & B \\ C\end{array}\right) \in \mathfrak{g l}\left(2, \mathbb{H}_{\mathbb{C}}\right)$, then $\pi_{d l}\left(\begin{array}{ll}A & B \\ C & D\end{array}\right) f \in \mathcal{D} \mathcal{R}$ and $\pi_{d r}\left(\begin{array}{ll}A & B \\ C & D\end{array}\right) g \in \mathcal{D} \mathcal{R}^{\prime}$. Consider, for example, the case of $\pi_{d l}\left(\begin{array}{ll}0 & 0 \\ C & 0\end{array}\right) f$, the other cases are similar. We have:

$$
\begin{array}{r}
\left(\nabla^{+} \otimes 1\right) \pi_{d l}\left(\begin{array}{ll}
0 & 0 \\
C & 0
\end{array}\right) f=\left(\nabla^{+} \otimes 1\right)(\operatorname{Tr}(Z C Z \partial+C Z) f+(C Z \otimes 1) f)+(1 \otimes C Z)\left(\nabla^{+} \otimes 1\right) f \\
+(1 \otimes C)\left(e_{0} \otimes e_{0}+e_{1} \otimes e_{1}+e_{2} \otimes e_{2}+e_{3} \otimes e_{3}\right) f
\end{array}
$$

the first summand is zero essentially because the space of left regular functions is invariant under the action $\pi_{l}$ (equation (22) in [FL1]), the second summand is zero because $f$ satisfies $\left(\nabla^{+} \otimes 1\right) f=0$, and the third summand is zero by Lemma 5 .

Lemma 5. Let $t \in \mathbb{S} \odot \mathbb{S}$ and $t^{\prime} \in \mathbb{S}^{\prime} \odot \mathbb{S}^{\prime}$, then

$$
\left(e_{0} \otimes e_{0}+e_{1} \otimes e_{1}+e_{2} \otimes e_{2}+e_{3} \otimes e_{3}\right) t=0 \quad \text { in } \mathbb{S} \otimes \mathbb{S}
$$

and

$$
t^{\prime}\left(e_{0} \otimes e_{0}+e_{1} \otimes e_{1}+e_{2} \otimes e_{2}+e_{3} \otimes e_{3}\right)=0 \quad \text { in } \mathbb{S}^{\prime} \otimes \mathbb{S}^{\prime}
$$

Proof. Under the standard realization of $\mathbb{H}$ as a subalgebra of $\mathbb{C}^{2 \times 2}$, we have:

$$
e_{0}=\left(\begin{array}{ll}
1 & 0 \\
0 & 1
\end{array}\right), \quad e_{1}=\left(\begin{array}{cc}
0 & -i \\
-i & 0
\end{array}\right), \quad e_{2}=\left(\begin{array}{cc}
0 & -1 \\
1 & 0
\end{array}\right), \quad e_{3}=\left(\begin{array}{cc}
-i & 0 \\
0 & i
\end{array}\right) .
$$

Then by direct computation using Kronecker product (see also Subsection 2.3) we obtain

$$
e_{0} \otimes e_{0}+e_{1} \otimes e_{1}+e_{2} \otimes e_{2}+e_{3} \otimes e_{3}=\left(\begin{array}{cccc}
0 & 0 & 0 & 0 \\
0 & 2 & -2 & 0 \\
0 & -2 & 2 & 0 \\
0 & 0 & 0 & 0
\end{array}\right)
$$

Since the elements of $\mathbb{S} \odot \mathbb{S}$ and $\mathbb{S}^{\prime} \odot \mathbb{S}^{\prime}$, when realized as 4-tuples, have equal second and third entries, they are annihilated by the above matrix, and the result follows.

\subsection{Cauchy-Fueter Formulas for Doubly Regular Functions}

In this section we derive Cauchy-Fueter type formulas for doubly regular functions from the classical Cauchy-Fueter formulas for left and right regular functions.

Lemma 6. Let $f(Z)$ be a doubly left regular function, then the $\mathbb{S} \otimes \mathbb{S}$-valued functions $(1 \otimes Z) f(Z)$ and $(Z \otimes 1) f(Z)$ are "left regular" in the sense that they satisfy

$$
\left(\nabla^{+} \otimes 1\right)[(1 \otimes Z) f(Z)]=0, \quad\left(1 \otimes \nabla^{+}\right)[(Z \otimes 1) f(Z)]=0 .
$$

Similarly, if $g(Z)$ is a doubly right regular function, then the $\mathbb{S}^{\prime} \otimes \mathbb{S}^{\prime}$-valued functions $g(Z)(1 \otimes$ $Z)$ and $g(Z)(Z \otimes 1)$ are "right regular" in the sense that they satisfy

$$
[g(Z)(1 \otimes Z)]\left(\overleftarrow{\nabla^{+} \otimes 1}\right)=0, \quad[g(Z)(Z \otimes 1)]\left(\overleftarrow{1 \otimes \nabla^{+}}\right)=0
$$

Proof. We have:

$\left(\nabla^{+} \otimes 1\right)[(1 \otimes Z) f(Z)]=\left(e_{0} \otimes e_{0}+e_{1} \otimes e_{1}+e_{2} \otimes e_{2}+e_{3} \otimes e_{3}\right) f(Z)+(1 \otimes Z)\left[\left(\nabla^{+} \otimes 1\right) f(Z)\right]$, the first summand is zero by Lemma [5] and the second summand is zero because $f$ satisfies $\left(\nabla^{+} \otimes 1\right) f=0$. Proofs of the other assertions are similar. 
Let $(\mathrm{deg}+2)$ denote the degree operator plus two times the identity. For example, if $f$ is a function on $\mathbb{H}$,

$$
(\operatorname{deg}+2) f=x^{0} \frac{\partial f}{\partial x^{0}}+x^{1} \frac{\partial f}{\partial x^{1}}+x^{2} \frac{\partial f}{\partial x^{2}}+x^{3} \frac{\partial f}{\partial x^{3}}+2 f .
$$

Similarly, we can define operators deg and $(\operatorname{deg}+2)$ acting on functions on $\mathbb{H}_{\mathbb{C}}$. For convenience we recall Lemma 8 from [FL2] (it applies to both cases).

\section{Lemma 7.}

$$
2(\operatorname{deg}+2)=Z^{+} \nabla^{+}+\nabla Z=\nabla^{+} Z^{+}+Z \nabla .
$$

Define

$$
k_{1}(Z-W)=\frac{1}{4}(\nabla \otimes \nabla)\left(\frac{1}{N(Z-W)}\right)
$$

(the derivatives can be taken with respect to either $Z$ or $W$ variable - the result is the same); this is a function of $Z$ and $W$ taking values in $\mathbb{H}_{\mathbb{C}} \otimes \mathbb{H}_{\mathbb{C}}$, it is spelled out in equation (19). We also consider holomorphic 3 -forms $D z \otimes Z$ and $Z \otimes D z$ on $\mathbb{H}_{\mathbb{C}}$ with values in $\mathbb{H}_{\mathbb{C}} \otimes \mathbb{H}_{\mathbb{C}}$. Then we obtain the following analogue of the Cauchy-Fueter formulas for doubly regular functions.

Theorem 8. Let $U \subset \mathbb{H}$ be an open bounded subset with piecewise $\mathcal{C}^{1}$ boundary $\partial U$. Suppose that $f(Z)$ is doubly left regular on a neighborhood of the closure $\bar{U}$, then

$$
\begin{aligned}
\frac{1}{2 \pi^{2}} \int_{\partial U} k_{1}(Z-W) & \cdot(D z \otimes Z) \cdot f(Z) \\
& =\frac{1}{2 \pi^{2}} \int_{\partial U} k_{1}(Z-W) \cdot(Z \otimes D z) \cdot f(Z)= \begin{cases}(\operatorname{deg}+2) f(W) & \text { if } W \in U ; \\
0 & \text { if } W \notin \bar{U} .\end{cases}
\end{aligned}
$$

If $g(Z)$ is doubly right regular on a neighborhood of the closure $\bar{U}$, then

$$
\begin{aligned}
\frac{1}{2 \pi^{2}} \int_{\partial U} g(Z) \cdot(D z & \otimes Z) \cdot k_{1}(Z-W) \\
& =\frac{1}{2 \pi^{2}} \int_{\partial U} g(Z) \cdot(Z \otimes D z) \cdot k_{1}(Z-W)= \begin{cases}(\operatorname{deg}+2) g(W) & \text { if } W \in U ; \\
0 & \text { if } W \notin \bar{U}\end{cases}
\end{aligned}
$$

Proof. By Lemma 6, the $\mathbb{S} \otimes \mathbb{S}$-valued function $(1 \otimes Z) f(Z)$ satisfies $\left(\nabla^{+} \otimes 1\right)[(1 \otimes Z) f(Z)]=0$. From the classical Cauchy-Fueter formula for left regular functions, we obtain:

$$
\frac{1}{2 \pi^{2}} \int_{\partial U} k_{1 / 2}(Z-W) \cdot(D z \otimes 1) \cdot(1 \otimes Z) f(Z)= \begin{cases}(1 \otimes W) f(W) & \text { if } W \in U \\ 0 & \text { if } W \notin \bar{U}\end{cases}
$$

where

$$
k_{1 / 2}(Z-W)=\frac{(Z-W)^{-1}}{N(Z-W)} \otimes 1=-\frac{1}{2}\left(\nabla_{Z} \otimes 1\right)\left(\frac{1}{N(Z-W)}\right)=\frac{1}{2}\left(\nabla_{W} \otimes 1\right)\left(\frac{1}{N(Z-W)}\right) .
$$

Applying $1 \otimes \nabla$ to both sides of (17) (the derivative is taken with respect to $W$ ),

$$
\begin{aligned}
\frac{1}{\pi^{2}} \int_{\partial U} k_{1}(Z-W) \cdot(D z \otimes Z) \cdot f(Z)= \begin{cases}(1 \otimes \nabla)(1 \otimes W) f(W) & \text { if } W \in U ; \\
0 & \text { if } W \notin \bar{U}\end{cases} \\
= \begin{cases}2(\operatorname{deg}+2) f(W) & \text { if } W \in U ; \\
0 & \text { if } W \notin \bar{U},\end{cases}
\end{aligned}
$$

where the last equality follows from (15), since $\left(1 \otimes \nabla^{+}\right) f=0$. The other cases are similar. 
We have an analogue of Liouville's theorem for doubly regular functions:

Corollary 9. Let $f: \mathbb{H} \rightarrow \mathbb{S} \odot \mathbb{S}$ be a function that is doubly left regular and bounded on $\mathbb{H}$, then $f$ is constant. Similarly, if $g: \mathbb{H} \rightarrow \mathbb{S}^{\prime} \odot \mathbb{S}^{\prime}$ is a function that is doubly right regular and bounded on $\mathbb{H}$, then $g$ is constant.

Proof. The proof is essentially the same as for the (classical) left and right regular functions on $\mathbb{H}$, so we only give a sketch of the first part. From Theorem 8 we have:

$$
\frac{\partial}{\partial x_{11}}(\operatorname{deg}+2) f(X)=\frac{1}{2 \pi^{2}} \int_{S_{R}^{3}} \frac{\partial k_{1}(Z-X)}{\partial x_{11}} \cdot(D z \otimes Z) \cdot f(Z)
$$

where $S_{R}^{3} \subset \mathbb{H}$ is the three-dimensional sphere of radius $R$ centered at the origin

$$
S_{R}^{3}=\left\{X \in \mathbb{H} ; N(X)=R^{2}\right\}
$$

with $R^{2}>N(X)$. If $f$ is bounded, one easily shows that the integral on the right hand side tends to zero as $R \rightarrow \infty$. Thus $\frac{\partial}{\partial x_{11}}(\operatorname{deg}+2) f=0$. Similarly,

$$
\frac{\partial}{\partial x_{12}}(\operatorname{deg}+2) f=\frac{\partial}{\partial x_{21}}(\operatorname{deg}+2) f=\frac{\partial}{\partial x_{22}}(\operatorname{deg}+2) f=0 .
$$

It follows that $(\operatorname{deg}+2) f$ and hence $f$ are constant.

\subsection{Expansion of the Cauchy-Fueter Kernel for Doubly Regular Functions}

We often identify $\mathbb{H}_{\mathbb{C}}$ with $2 \times 2$ matrices with complex entries. Similarly, it will be convenient to identify $\mathbb{H}_{\mathbb{C}} \otimes \mathbb{H}_{\mathbb{C}}$ with $4 \times 4$ matrices with complex entries using the Kronecker product. Let $\mathbb{C}^{n \times n}$ denote the algebra of $n \times n$ complex matrices. If $A=\left(\begin{array}{ll}a_{11} & a_{12} \\ a_{21} & a_{22}\end{array}\right), B=\left(\begin{array}{ll}b_{11} & b_{12} \\ b_{21} & b_{22}\end{array}\right) \in \mathbb{C}^{2 \times 2}$, then their Kronecker product is

$$
A \otimes B=\left(\begin{array}{ll}
a_{11} B & a_{12} B \\
a_{21} B & a_{22} B
\end{array}\right)=\left(\begin{array}{llll}
a_{11} b_{11} & a_{11} b_{12} & a_{12} b_{11} & a_{12} b_{12} \\
a_{11} b_{21} & a_{11} b_{22} & a_{12} b_{21} & a_{12} b_{22} \\
a_{21} b_{11} & a_{21} b_{12} & a_{22} b_{11} & a_{22} b_{12} \\
a_{21} b_{21} & a_{21} b_{22} & a_{22} b_{21} & a_{22} b_{22}
\end{array}\right) \in \mathbb{C}^{4 \times 4} .
$$

Similarly, if we identify $\mathbb{S}$ and $\mathbb{S}^{\prime}$ with columns and rows of two complex numbers respectively, then

$$
\left(\begin{array}{l}
z_{1} \\
z_{2}
\end{array}\right) \otimes\left(\begin{array}{l}
w_{1} \\
w_{2}
\end{array}\right)=\left(\begin{array}{c}
z_{1} w_{1} \\
z_{1} w_{2} \\
z_{2} w_{1} \\
z_{2} w_{2}
\end{array}\right), \quad\left(z_{1}, z_{2}\right) \otimes\left(w_{1}, w_{2}\right)=\left(z_{1} w_{1}, z_{1} w_{2}, z_{2} w_{1}, z_{2} w_{2}\right)
$$

A 4-tuple belongs to $\mathbb{S} \odot \mathbb{S}$ or $\mathbb{S}^{\prime} \odot \mathbb{S}^{\prime}$ if and only if its second entry equals the third entry. It is easy to see that Kronecker product satisfies

$$
(A \otimes B)(C \otimes D)=(A C) \otimes(B D) .
$$

Recall that the Cauchy-Fueter kernel for doubly regular functions is defined by (16). From its realization as a $4 \times 4$ matrix, we find:

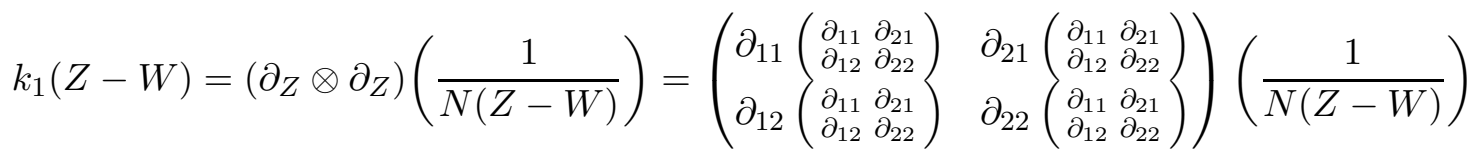

$$
\begin{aligned}
& =\frac{2}{N(Z-W)}(Z-W)^{-1} \otimes(Z-W)^{-1} \\
& -\frac{1 / 2}{N(Z-W)^{2}}\left(e_{0} \otimes e_{0}+e_{1} \otimes e_{1}+e_{2} \otimes e_{2}+e_{3} \otimes e_{3}\right) .
\end{aligned}
$$


Next we recall the matrix coefficients $t_{n \underline{m}}^{l}(Z)$ 's of $S U(2)$ described by equation (27) of [FL1] (cf. [V]):

$$
t_{n \underline{m}}^{l}(Z)=\frac{1}{2 \pi i} \oint\left(s z_{11}+z_{21}\right)^{l-m}\left(s z_{12}+z_{22}\right)^{l+m} s^{-l+n} \frac{d s}{s}, \quad \begin{gathered}
l=0, \frac{1}{2}, 1, \frac{3}{2}, \ldots, \\
m, n \in \mathbb{Z}+l, \\
-l \leq m, n \leq l,
\end{gathered},
$$

$Z=\left(\begin{array}{cc}{ }_{11} & z_{12} \\ z_{21} & z_{22}\end{array}\right) \in \mathbb{H}_{\mathbb{C}}$, the integral is taken over a loop in $\mathbb{C}$ going once around the origin in the counterclockwise direction. We regard these functions as polynomials on $\mathbb{H}_{\mathbb{C}}$. Using Lemma 22 from [FL1] repeatedly, we compute:

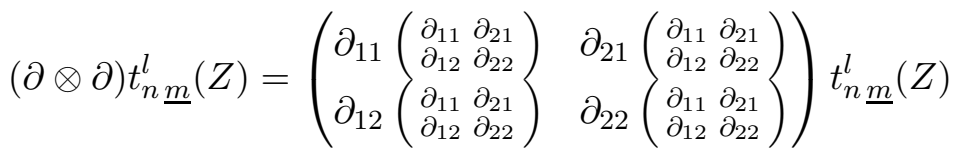

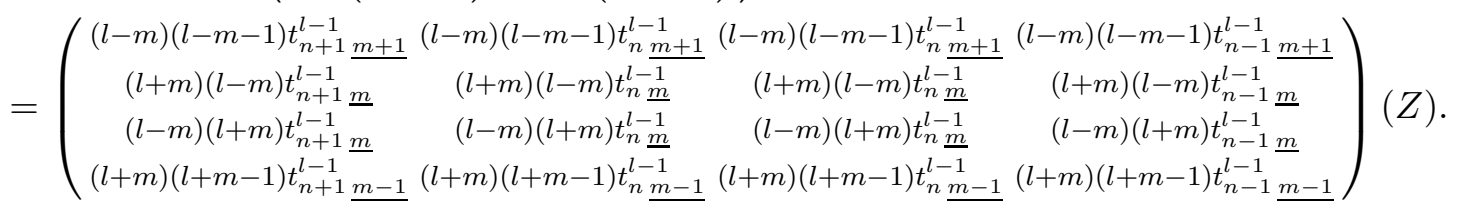

Since $\square t_{n \underline{m}}^{l}(Z)=0$, by observation made after Definition 1, the columns and rows of this $4 \times 4$ matrix are respectively doubly left and right regular.

Lemma 10. Let

$$
l=0, \frac{1}{2}, 1, \frac{3}{2}, \ldots, \quad m, n \in \mathbb{Z}+l, \quad-l-1 \leq m \leq l+1, \quad-l \leq n \leq l .
$$

The functions $\mathbb{H}_{\mathbb{C}} \rightarrow \mathbb{S} \odot \mathbb{S}$

$$
F_{l, m, n}(Z)=\left(\begin{array}{c}
(l-m)(l-m+1) t_{n}^{l}{ }_{n+1}^{m+1}(Z) \\
(l-m+1)(l+m+1) t_{n \underline{m}}^{l}(Z) \\
(l-m+1)(l+m+1) t_{n \underline{m}}^{l}(Z) \\
(l+m)(l+m+1) t_{n \underline{m-1}}^{l}(Z)
\end{array}\right)
$$

and

$$
F_{l, m, n}^{\prime}(Z)=\frac{1}{N(Z)}\left(\begin{array}{c}
(l-n+1)(l-n+2) t_{n-1}^{l+1}\left(Z^{-1}\right) \\
(l-n+1)(l+n+1) t_{n}^{l+1}\left(Z^{-1}\right) \\
(l-n+1)(l+n+1) t_{n \underline{m}}^{l+1}\left(Z^{-1}\right) \\
(l+n+1)(l+n+2) t_{n+1}^{l+1}\left(Z^{-1}\right)
\end{array}\right)
$$

are doubly left regular. Similarly, the functions $\mathbb{H}_{\mathbb{C}} \rightarrow \mathbb{S}^{\prime} \odot \mathbb{S}^{\prime}$

$$
G_{l, m, n}(Z)=\left(t_{m+1 \underline{n}}^{l}(Z), t_{m \underline{n}}^{l}(Z), t_{m \underline{n}}^{l}(Z), t_{m-1 \underline{n}}^{l}(Z)\right)
$$

and

$$
G_{l, m, n}^{\prime}(Z)=N(Z)^{-1} \cdot\left(t_{m \underline{n-1}}^{l+1}\left(Z^{-1}\right), t_{m \underline{n}}^{l+1}\left(Z^{-1}\right), t_{m \underline{n}}^{l+1}\left(Z^{-1}\right), t_{m \underline{n+1}}^{l+1}\left(Z^{-1}\right)\right)
$$

are doubly right regular.

Proof. The result can be derived either by direct computations using Lemmas 22 and 23 in [FL1] or from Proposition 24 in [FL1].

Proposition 11. Let

$$
\mathcal{F}^{+}=\mathbb{C} \text {-span of }\left\{F_{l, m, n}(Z)\right\}, \quad \mathcal{F}^{-}=\mathbb{C} \text {-span of }\left\{F_{l, m, n}^{\prime}(Z)\right\},
$$




$$
\begin{gathered}
\mathcal{G}^{+}=\mathbb{C} \text {-span of }\left\{G_{l, m, n}(Z)\right\}, \quad \mathcal{G}^{-}=\mathbb{C} \text {-span of }\left\{G_{l, m, n}^{\prime}(Z)\right\}, \\
l=0, \frac{1}{2}, 1, \frac{3}{2}, \ldots, \quad m, n \in \mathbb{Z}+l, \quad-l-1 \leq m \leq l+1, \quad-l \leq n \leq l .
\end{gathered}
$$

Then $\left(\pi_{d l}, \mathcal{F}^{+}\right),\left(\pi_{d l}, \mathcal{F}^{-}\right),\left(\pi_{d r}, \mathcal{G}^{+}\right)$and $\left(\pi_{d r}, \mathcal{G}^{-}\right)$are irreducible representations of $\mathfrak{s l}\left(2, \mathbb{H}_{\mathbb{C}}\right)$ (as well as $\mathfrak{g l}\left(2, \mathbb{H}_{\mathbb{C}}\right)$ ).

The result can be proved directly by finding the $K$-types of $\left(\pi_{d l}, \mathcal{F}^{ \pm}\right)$and $\left(\pi_{d r}, \mathcal{G}^{ \pm}\right)$, computing the actions of $\left(\begin{array}{ll}0 & B \\ 0 & 0\end{array}\right)$ and $\left(\begin{array}{ll}0 & 0 \\ C & 0\end{array}\right)$, then showing that any non-zero vector generates the whole space. Alternatively, it follows from Corollary 51 .

Next we derive two matrix coefficient expansions of the Cauchy-Fueter kernel for doubly regular functions (16) in terms of these functions $F_{l, m, n}, F_{l, m, n}^{\prime}, G_{l, m, n}, G_{l, m, n}^{\prime}$. This is a doubly regular function analogue of Proposition 26 from [FL1] for the usual regular functions (see also Proposition 113).

Proposition 12. We have the following expansions

$$
\begin{aligned}
& k_{1}(Z-W)=\sum_{l, m, n} F_{l, m, n}(Z) \cdot G_{l, m, n}^{\prime}(W)=\frac{1}{N(W)} \sum_{l, m, n}\left(\begin{array}{c}
(l-m)(l-m+1) t_{n}^{l} \underline{m+1}_{(Z)}^{l}(Z-m+1)(l+m+1) t_{n \underline{m}}^{l}(Z) \\
(l-m+1)(l+m+1) t_{n \underline{m}}^{l}(Z) \\
(l+m)(l+m+1) t_{n \underline{m-1}}^{l}(Z)
\end{array}\right) \\
& \times\left(t_{m \underline{n-1}}^{l+1}\left(W^{-1}\right), t_{m \underline{n}}^{l+1}\left(W^{-1}\right), t_{m \underline{n}}^{l+1}\left(W^{-1}\right), t_{m \underline{n+1}}^{l+1}\left(W^{-1}\right)\right),
\end{aligned}
$$

which converges uniformly on compact subsets in the region $\left\{(Z, W) \in \mathbb{H}_{\mathbb{C}} \times \mathbb{H}_{\mathbb{C}}^{\times} ; Z W^{-1} \in \mathbb{D}^{+}\right\}$, and

$$
\begin{aligned}
k_{1}(Z-W)=\sum_{l, m, n} F_{l . m, n}^{\prime}(Z) \cdot G_{l, m, n}(W) & =\frac{1}{N(Z)} \sum_{l, m, n}\left(\begin{array}{c}
(l-n+1)(l-n+2) t_{n-1}^{l} \\
(l-n+1)(l+n+1) t_{n}^{l} \underline{m}\left(Z^{-1}\right) \\
(l-n+1)(l+n+1) t_{n \underline{m}}^{l}\left(Z^{-1}\right) \\
(l+n+1)(l+n+2) t_{n+1 \underline{m}}^{l}\left(Z^{-1}\right)
\end{array}\right) \\
& \times\left(t_{m+1}^{l} \underline{n}(W), t_{m \underline{n}}^{l}(W), t_{m \underline{n}}^{l}(W), t_{m-1}^{l} \underline{\underline{n}}(W)\right), \quad(24)
\end{aligned}
$$

which converges uniformly on compact subsets in the region $\left\{(Z, W) \in \mathbb{H}_{\mathbb{C}}^{\times} \times \mathbb{H}_{\mathbb{C}} ; W Z^{-1} \in \mathbb{D}^{+}\right\}$. The sums are taken first over all $m=-l-1,-l, \ldots, l+1, n=-l,-l+1, \ldots, l$, then over $l=0, \frac{1}{2}, 1, \frac{3}{2}, \ldots$.

Proof. See the discussion after equation (21) in [FL3] for the definition of the open domain $\mathbb{D} \subset \mathbb{H}_{\mathbb{C}}$. Using our previous calculations (19) and Proposition 26 in [FL1] (see also Proposition 113), we obtain:

$$
\begin{aligned}
& k_{1}(Z-W)=\left(\partial_{Z} \otimes \partial_{Z}\right)\left(\frac{1}{N(Z-W)}\right)=-\left(\partial_{Z} \otimes 1\right)\left(1 \otimes \frac{(Z-W)^{-1}}{N(Z-W)}\right) \\
& =\frac{1}{N(W)}\left(\partial_{Z} \otimes 1\right)\left(\begin{array}{r}
1 \otimes \sum_{l, m, n^{\prime}}\left(\begin{array}{c}
(l-m+1) t_{n^{\prime}}^{l+\frac{1}{2}}(Z)+\frac{1}{2} \\
(l+m+1) t_{n^{\prime}}^{l+\frac{1}{2}}\left(Z-\frac{1}{2}\right.
\end{array}\right) \cdot(Z) \\
=\frac{1}{N(W)} \sum_{l, m, n^{\prime}}^{l+1}\left(\begin{array}{ll}
A_{11}\left(l, m, n^{\prime}\right. \\
A_{21}\left(l, m, n^{\prime}\right) & A_{12}\left(l, m, n^{\prime}\right) \\
A_{22}\left(l, m, n^{\prime}\right)
\end{array}\right),
\end{array}\right.
\end{aligned}
$$


where $l=-\frac{1}{2}, 0, \frac{1}{2}, 1, \frac{3}{2}, \ldots, m=-l-1,-l, \ldots, l+1, n^{\prime}=-l-\frac{1}{2},-l+\frac{1}{2}, \ldots, l+\frac{1}{2}$, and

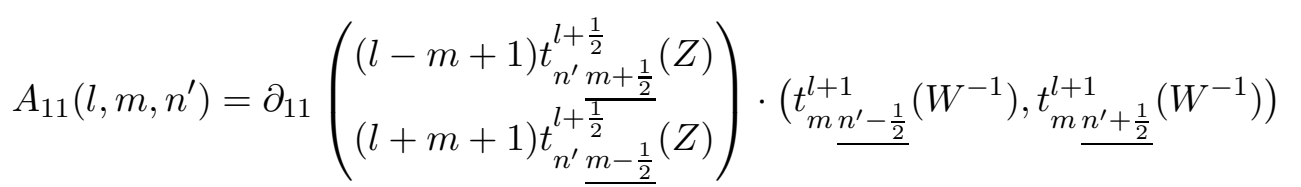

$$
\begin{aligned}
& =\left(\begin{array}{c}
(l-m)(l-m+1) t_{n^{\prime}+\frac{1}{2} \underline{m+1}}^{l}(Z) \\
(l-m+1)(l+m+1) t_{n^{\prime}+\frac{1}{2} \underline{m}}^{(Z)}
\end{array}\right) \cdot\left(t_{m \underline{n^{\prime}-\frac{1}{2}}}^{l+1}\left(W^{-1}\right), t_{m \underline{n^{\prime}+\frac{1}{2}}}^{l+1}\left(W^{-1}\right)\right), \\
& A_{12}\left(l, m, n^{\prime}\right)=\partial_{21}\left(\begin{array}{c}
(l-m+1) t_{n^{\prime} \frac{m+\frac{1}{2}}{2+\frac{1}{2}}}^{l+\frac{1}{2}}(Z) \\
(l+m+1) t_{n^{\prime} \underline{\frac{m-\frac{1}{2}}{2}}}^{l+m}(Z)
\end{array}\right) \cdot\left(t_{m \underline{n^{\prime}-\frac{1}{2}}}^{l+1}\left(W^{-1}\right), t_{\underline{m n^{\prime}+\frac{1}{2}}}^{l+1}\left(W^{-1}\right)\right) \\
& =\left(\begin{array}{c}
(l-m)(l-m+1) t_{n^{\prime}-\frac{1}{2} \underline{m+1}}^{l}(Z) \\
(l-m+1)(l+m+1) t_{n^{\prime}-\frac{1}{2} \underline{m}}^{l}(Z)
\end{array}\right) \cdot\left(t_{m \underline{n^{\prime}-\frac{1}{2}}}^{l+1}\left(W^{-1}\right), t_{m \underline{n^{\prime}+\frac{1}{2}}}^{l+1}\left(W^{-1}\right)\right), \\
& A_{21}\left(l, m, n^{\prime}\right)=\partial_{12}\left(\begin{array}{c}
(l-m+1) t_{n^{\prime} m+\frac{1}{2}}^{l+\frac{1}{2}}(Z) \\
(l+m+1) t_{n^{\prime} m-\frac{1}{2}}^{l+\frac{1}{2}}(Z)
\end{array}\right) \cdot\left(t_{m \underline{n^{\prime}-\frac{1}{2}}}^{l+1}\left(W^{-1}\right), t_{\frac{m n^{\prime}+\frac{1}{2}}{l+1}}^{l}\left(W^{-1}\right)\right) \\
& =\left(\begin{array}{c}
(l-m+1)(l+m+1) t_{n^{\prime}+\frac{1}{2} \underline{m}}^{l}(Z) \\
(l+m)(l+m+1) t_{n^{\prime}+\frac{1}{2} \underline{m-1}}^{l}(Z)
\end{array}\right) \cdot\left(t_{m \underline{n^{\prime}-\frac{1}{2}}}^{l+1}\left(W^{-1}\right), t_{m \underline{n^{\prime}+\frac{1}{2}}}^{l+1}\left(W^{-1}\right)\right), \\
& A_{22}\left(l, m, n^{\prime}\right)=\partial_{22}\left(\begin{array}{c}
(l-m+1) t_{n^{\prime} m+\frac{1}{2}}^{l+\frac{1}{2}}(Z) \\
(l+m+1) t_{n^{\prime} \frac{m-\frac{1}{2}}{2}}^{l+\frac{1}{2}}(Z)
\end{array}\right) \cdot\left(t_{m \underline{n^{\prime}-\frac{1}{2}}}^{l+1}\left(W^{-1}\right), t_{\underline{m} \underline{n^{\prime}+\frac{1}{2}}}^{l+1}\left(W^{-1}\right)\right) \\
& =\left(\begin{array}{c}
(l-m+1)(l+m+1) t_{n^{\prime}-\frac{1}{2} \underline{m}}^{l}(Z) \\
(l+m)(l+m+1) t_{n^{\prime}-\frac{1}{2} \underline{m-1}}^{l}(Z)
\end{array}\right) \cdot\left(t_{m \underline{n^{\prime}-\frac{1}{2}}}^{l+1}\left(W^{-1}\right), t_{m \underline{n^{\prime}+\frac{1}{2}}}^{l+1}\left(W^{-1}\right)\right) .
\end{aligned}
$$

Since the terms with $l=-\frac{1}{2}$ are zero, we can restrict $l$ to $0, \frac{1}{2}, 1, \frac{3}{2}, \ldots$ We have:

$$
\begin{aligned}
& \sum_{l, m, n^{\prime}} A_{11}\left(l, m, n^{\prime}\right)=\sum_{l, m, n}\left(\begin{array}{c}
(l-m)(l-m+1) t_{n \underline{m+1}}^{l}(Z) \\
(l-m+1)(l+m+1) t_{n \underline{m}}^{l}(Z)
\end{array}\right) \cdot\left(t_{m \underline{n-1}}^{l+1}\left(W^{-1}\right), t_{m \underline{n}}^{l+1}\left(W^{-1}\right)\right), \\
& \sum_{l, m, n^{\prime}} A_{21}\left(l, m, n^{\prime}\right)=\sum_{l, m, n}\left(\begin{array}{c}
(l-m+1)(l+m+1) t_{n \underline{m}}^{l}(Z) \\
(l+m)(l+m+1) t_{n \underline{m-1}}^{l}(Z)
\end{array}\right) \cdot\left(t_{m \underline{n-1}}^{l+1}\left(W^{-1}\right), t_{m \underline{n}}^{l+1}\left(W^{-1}\right)\right),
\end{aligned}
$$

where $n=n^{\prime}+\frac{1}{2}$, and

$$
\begin{aligned}
& \sum_{l, m, n^{\prime}} A_{12}\left(l, m, n^{\prime}\right)=\sum_{l, m, n}\left(\begin{array}{c}
(l-m)(l-m+1) t_{n \underline{m+1}}^{l}(Z) \\
(l-m+1)(l+m+1) t_{n \underline{m}}^{l}(Z)
\end{array}\right) \cdot\left(t_{m \underline{\underline{n}}}^{l+1}\left(W^{-1}\right), t_{m \underline{n+1}}^{l+1}\left(W^{-1}\right)\right), \\
& \sum_{l, m, n^{\prime}} A_{22}\left(l, m, n^{\prime}\right)=\sum_{l, m, n}\left(\begin{array}{c}
(l-m+1)(l+m+1) t_{n \underline{m}}^{l}(Z) \\
(l+m)(l+m+1) t_{n \underline{m-1}}^{l}(Z)
\end{array}\right) \cdot\left(t_{m \underline{\underline{n}}}^{l+1}\left(W^{-1}\right), t_{m \underline{n+1}}^{l+1}\left(W^{-1}\right)\right),
\end{aligned}
$$

where $n=n^{\prime}-\frac{1}{2}$; in all cases $n=-l,-l+1, \ldots, l$. This proves the first expansion. The other expansion is proved similarly. 


\subsection{Doubly Regular Functions on $\mathbb{H}^{\times}$}

In this subsection we show that if a (left or right) doubly regular function is defined on all of $\mathbb{H}^{\times}$, then the operator $(\operatorname{deg}+2)$ can be inverted. This will be needed, for example, when we define the invariant bilinear pairing for such functions.

We start with a doubly left regular function $f: \mathbb{H}^{\times} \rightarrow \mathbb{S} \odot \mathbb{S}$ and derive some properties of such functions. Of course, doubly right regular functions $g: \mathbb{H}^{\times} \rightarrow \mathbb{S}^{\prime} \odot \mathbb{S}^{\prime}$ have similar properties. Let $0<r<R$, then, by the Cauchy-Fueter formulas for doubly regular functions (Theorem 8),

$$
(\operatorname{deg}+2) f(W)=\frac{1}{2 \pi^{2}} \int_{S_{R}^{3}} k_{1}(Z-W) \cdot(D z \otimes Z) \cdot f(Z)-\frac{1}{2 \pi^{2}} \int_{S_{r}^{3}} k_{1}(Z-W) \cdot(D z \otimes Z) \cdot f(Z),
$$

for all $W \in \mathbb{H}$ such that $r^{2}<N(W)<R^{2}$, where $S_{R}^{3} \subset \mathbb{H}$ is the sphere of radius $R$ centered at the origin

$$
S_{R}^{3}=\left\{X \in \mathbb{H} ; N(X)=R^{2}\right\} .
$$

Define functions $\tilde{f}_{+}: \mathbb{H} \rightarrow \mathbb{S} \odot \mathbb{S}$ and $\tilde{f}_{-}: \mathbb{H}^{\times} \rightarrow \mathbb{S} \odot \mathbb{S}$ by

$$
\begin{array}{ll}
\tilde{f}_{+}(W)=\frac{1}{2 \pi^{2}} \int_{S_{R}^{3}} k_{1}(Z-W) \cdot(D z \otimes Z) \cdot f(Z), & R^{2}>N(W), \\
\tilde{f}_{-}(W)=-\frac{1}{2 \pi^{2}} \int_{S_{r}^{3}} k_{1}(Z-W) \cdot(D z \otimes Z) \cdot f(Z), & r^{2}<N(W) .
\end{array}
$$

Note that $\tilde{f}_{+}$and $\tilde{f}_{-}$are doubly left regular and that $\tilde{f}_{-}(W)$ decays at infinity at a rate $\sim N(W)^{-2}$.

For a function $\varphi$ defined on $\mathbb{H}$ or, slightly more generally, on a star-shaped open subset of $\mathbb{H}$ centered at the origin, let

$$
\left((\operatorname{deg}+2)^{-1} \varphi\right)(Z)=\int_{0}^{1} t \cdot \varphi(t Z) d t .
$$

Similarly, for a function $\varphi$ defined on $\mathbb{H}^{\times}$and decaying sufficiently fast at infinity, we can define $(\operatorname{deg}+2)^{-1} \varphi$ as

$$
\left((\operatorname{deg}+2)^{-1} \varphi\right)(Z)=-\int_{1}^{\infty} t \cdot \varphi(t Z) d t
$$

Then

$$
(\operatorname{deg}+2)\left((\operatorname{deg}+2)^{-1} \varphi\right)=(\operatorname{deg}+2)^{-1}((\operatorname{deg}+2) \varphi)=\varphi
$$

for functions $\varphi$ that are either defined on star-shaped open subsets of $\mathbb{H}$ centered at the origin or on $\mathbb{H}^{\times}$and decaying sufficiently fast at infinity. (In the same fashion one can also define $(\operatorname{deg}+2)^{-1} \varphi$ for functions defined on star-shaped open subsets of $\mathbb{H}_{\mathbb{C}}$ centered at the origin or on $\mathbb{H}_{\mathbb{C}}^{\times}$and decaying sufficiently fast at infinity.)

We introduce functions

$$
f_{+}=(\operatorname{deg}+2)^{-1} \tilde{f}_{+} \quad \text { and } \quad f_{-}=(\operatorname{deg}+2)^{-1} \tilde{f}_{-} .
$$

Proposition 13. Let $f: \mathbb{H}^{\times} \rightarrow \mathbb{S} \odot \mathbb{S}$ be a doubly left regular function. Then $f(X)=f_{+}(X)+$ $f_{-}(X)$, for all $X \in \mathbb{H}^{\times}$. 
Proof. Let $f_{-2}=f-f_{+}-f_{-}$, we want to show that $f_{-2} \equiv 0$. Note that $f_{-2}: \mathbb{H}^{\times} \rightarrow \mathbb{S} \odot \mathbb{S}$ is a doubly left regular function such that $(\operatorname{deg}+2) f_{-2} \equiv 0$, hence $f_{-2}$ is homogeneous of degree -2 . Let

$$
f_{-3}=\frac{\partial}{\partial x_{11}} f_{-2}, \quad f_{-3}: \mathbb{H}^{\times} \rightarrow \mathbb{S} \odot \mathbb{S},
$$

then $f_{-3}$ is a doubly left regular function that is homogeneous of degree -3 .

By the Cauchy-Fueter formulas for doubly regular functions (Theorem 8),

$$
\begin{aligned}
f_{-3}(X)=-\frac{1}{2 \pi^{2}} \int_{S_{R}^{3}} k_{1}(Z-X) \cdot(D z \otimes Z) \cdot f_{-3}(Z) & \\
& +\frac{1}{2 \pi^{2}} \int_{S_{r}^{3}} k_{1}(Z-X) \cdot(D z \otimes Z) \cdot f_{-3}(Z),
\end{aligned}
$$

where $R, r>0$ are such that $r^{2}<N(X)<R^{2}$. By Liouville's theorem (Corollary 9), the first integral defines a doubly left regular function on $\mathbb{H}$ that is either constant or unbounded. On the other hand, the second integral defines a doubly left regular function on $\mathbb{H}^{\times}$that decays at infinity at a rate $\sim N(W)^{-2}$. We conclude that $f_{-3} \equiv 0$, hence $f_{-2} \equiv 0$ as well.

Definition 14. Let $f: \mathbb{H}^{\times} \rightarrow \mathbb{S} \odot \mathbb{S}$ be a doubly left regular function. We define

$$
(\operatorname{deg}+2)^{-1} f=(\operatorname{deg}+2)^{-1} f_{+}+(\operatorname{deg}+2)^{-1} f_{-} .
$$

Similarly, we can define $(\operatorname{deg}+2)^{-1} g$ for doubly right regular functions $g: \mathbb{H}^{\times} \rightarrow \mathbb{S}^{\prime} \odot \mathbb{S}^{\prime}$.

From the previous discussion we immediately obtain:

Proposition 15. Let $f: \mathbb{H}^{\times} \rightarrow \mathbb{S} \odot \mathbb{S}$ be a doubly left regular function and $g: \mathbb{H}^{\times} \rightarrow \mathbb{S}^{\prime} \odot \mathbb{S}^{\prime}$ a doubly right regular function. Then

$$
\begin{aligned}
(\operatorname{deg}+2)\left((\operatorname{deg}+2)^{-1} f\right) & =(\operatorname{deg}+2)^{-1}((\operatorname{deg}+2) f)=f \\
(\operatorname{deg}+2)\left((\operatorname{deg}+2)^{-1} g\right) & =(\operatorname{deg}+2)^{-1}((\operatorname{deg}+2) g)=g .
\end{aligned}
$$

From the expansions of the Cauchy-Fueter kernel (23) and (24) we immediately obtain an analogue of Laurent series expansion for doubly regular functions.

Corollary 16. Let $f: \mathbb{H}^{\times} \rightarrow \mathbb{S} \odot \mathbb{S}$ be a doubly left regular function, write $f=f_{+}+f_{-}$as in the above proposition. Then the functions $f_{+}$and $f_{-}$can be expanded as series

$$
f_{+}(X)=\sum_{l}\left(\sum_{m, n} a_{l, m, n} F_{l, m, n}(X)\right), \quad f_{-}(X)=\sum_{l}\left(\sum_{m, n} b_{l, m, n} F_{l, m, n}^{\prime}(X)\right) .
$$

If $g: \mathbb{H}^{\times} \rightarrow \mathbb{S}^{\prime} \odot \mathbb{S}^{\prime}$ is a doubly right regular function, then it can be expressed as $g=g_{+}+g_{-}$ in a similar way, and the functions $g_{+}$and $g_{-}$can be expanded as series

$$
g_{+}(X)=\sum_{l}\left(\sum_{m, n} c_{l, m, n} G_{l, m, n}(X)\right), \quad g_{-}(X)=\sum_{l}\left(\sum_{m, n} d_{l, m, n} G_{l, m, n}^{\prime}(X)\right) .
$$
21.

Formulas expressing the coefficients $a_{l, m, n}, b_{l, m, n}, c_{l, m, n}$ and $d_{l, m, n}$ will be given in Corollary 


\subsection{Invariant Bilinear Pairing for Doubly Regular Functions}

We define a pairing between doubly left and right regular functions as follows. If $f(Z)$ and $g(Z)$ are doubly left and right regular functions on $\mathbb{H}_{\mathbb{C}}^{\times}$respectively, then by the results of the previous subsection $(\operatorname{deg}+2)^{-1} f$ and $(\operatorname{deg}+2)^{-1} g$ are well defined, and we set

$$
\langle f, g\rangle_{\mathcal{D R}}=\frac{1}{2 \pi^{2}} \int_{Z \in S_{R}^{3}} g(Z) \cdot(Z \otimes D z) \cdot\left((\operatorname{deg}+2)^{-1} f\right)(Z),
$$

where $S_{R}^{3} \subset \mathbb{H} \subset \mathbb{H}_{\mathbb{C}}$ is the sphere of radius $R$ centered at the origin

$$
S_{R}^{3}=\left\{X \in \mathbb{H} ; N(X)=R^{2}\right\} .
$$

Recall that by Lemma 6 in [FL1] the 3 -form $D z$ restricted to $S_{R}^{3}$ becomes $Z d S / R$, where $d S$ is the usual Euclidean volume element on $S_{R}^{3}$. Thus we can rewrite (25) as

$$
\begin{aligned}
\langle f, g\rangle_{\mathcal{D R}}=\frac{1}{2 \pi^{2}} \int_{Z \in S_{R}^{3}} g(Z) \cdot(Z \otimes Z) \cdot\left((\operatorname{deg}+2)^{-1} f\right)(Z) \frac{d S}{R} & \\
& =\frac{1}{2 \pi^{2}} \int_{Z \in S_{R}^{3}} g(Z) \cdot(D z \otimes Z) \cdot\left((\operatorname{deg}+2)^{-1} f\right)(Z) .
\end{aligned}
$$

Since $\left(1 \otimes \nabla^{+}\right)(\operatorname{deg}+2)^{-1} f=0$ and, by Lemma 6 , $[g(Z)(Z \otimes 1)]\left(\overleftarrow{1 \otimes \nabla^{+}}\right)=0$, the integrand of (25) is a closed 3 -form and the contour of integration can be continuously deformed. In particular, this pairing does not depend on the choice of $R>0$.

Proposition 17. If $f(Z)$ and $g(Z)$ are doubly left and right regular functions on $\mathbb{H}_{\mathbb{C}}^{\times}$respectively, then

$$
\begin{array}{r}
\langle f, g\rangle_{\mathcal{D R}}=\frac{1}{2 \pi^{2}} \int_{Z \in S_{R}^{3}} g(Z) \cdot(Z \otimes D z) \cdot\left((\operatorname{deg}+2)^{-1} f\right)(Z) \\
=-\frac{1}{2 \pi^{2}} \int_{Z \in S_{R}^{3}}\left((\operatorname{deg}+2)^{-1} g\right)(Z) \cdot(Z \otimes D z) \cdot f(Z) .
\end{array}
$$

Proof. Since the expression

$$
\int_{Z \in S_{R}^{3}} g(Z) \cdot(Z \otimes D z) \cdot f(Z)
$$

is independent of the choice of $R>0$, we have:

$$
\begin{aligned}
0=\left.\frac{d}{d t}\right|_{t=1} & \left(\int_{Z \in S_{t R}^{3}} g(Z) \cdot(Z \otimes D z) \cdot f(Z)\right) \\
= & \int_{Z \in S_{R}^{3}}(((\operatorname{deg}+2) g)(Z) \cdot(Z \otimes D z) \cdot f(Z)+g(Z) \cdot(Z \otimes D z) \cdot((\operatorname{deg}+2) f)(Z)) .
\end{aligned}
$$

From this (26) follows.

Corollary 18. If $f(Z)$ and $g(Z)$ are doubly left and right regular functions on $\mathbb{H}_{\mathbb{C}}$ respectively and $W \in \mathbb{D}_{R}^{+}$(open domains $\mathbb{D}_{R}^{ \pm}$were defined by equation (22) in [FL3]), the Cauchy-Fueter formulas for doubly regular functions (Theorem 8) can be rewritten as

$$
f(W)=\left\langle k_{1}(Z-W), f(Z)\right\rangle_{\mathcal{D R}} \quad \text { and } \quad g(W)=-\left\langle g(Z), k_{1}(Z-W)\right\rangle_{\mathcal{D R}} .
$$


We can rewrite the bilinear pairing (25) in a more symmetrical way. Let $0<r<R$ and $0<r_{1}<R<r_{2}$. Using the Cauchy-Fueter formulas for doubly regular functions (Theorem 8 ), substituting

$$
\begin{aligned}
g(Z)=\frac{1}{2 \pi^{2}} & \int_{W \in S_{r_{2}}^{3}}\left((\operatorname{deg}+2)^{-1} g\right)(W) \cdot(W \otimes D w) \cdot k_{1}(W-Z) \\
& \quad-\frac{1}{2 \pi^{2}} \int_{W \in S_{r_{1}}^{3}}\left((\operatorname{deg}+2)^{-1} g\right)(W) \cdot(W \otimes D w) \cdot k_{1}(W-Z), \quad Z \in \mathbb{D}_{r_{2}}^{+} \cap \mathbb{D}_{r_{1}}^{-},
\end{aligned}
$$

into (25) and shifting contours of integration, we obtain:

$$
\begin{aligned}
& \langle f, g\rangle_{\mathcal{D R}} \\
& =\frac{1}{4 \pi^{4}} \iint_{\substack{Z \in S^{3} \\
W \in S_{R}^{3}}}\left((\operatorname{deg}+2)^{-1} g\right)(W) \cdot(W \otimes D w) \cdot k_{1}(W-Z) \cdot(Z \otimes D z) \cdot\left((\operatorname{deg}+2)^{-1} f\right)(Z) \\
& -\frac{1}{4 \pi^{4}} \iint_{\substack{Z \in S_{R}^{3} \\
W \in S_{r}^{3}}}\left((\operatorname{deg}+2)^{-1} g\right)(W) \cdot(W \otimes D w) \cdot k_{1}(W-Z) \cdot(Z \otimes D z) \cdot\left((\operatorname{deg}+2)^{-1} f\right)(Z) .
\end{aligned}
$$

Proposition 19. The bilinear pairing (25) is $\mathfrak{g l}\left(2, \mathbb{H}_{\mathbb{C}}\right)$-invariant.

Proof. It is sufficient to show that the pairing is invariant under $S U(2) \times S U(2) \subset G L\left(2, \mathbb{H}_{\mathbb{C}}\right)$, dilation matrices $\left(\begin{array}{ll}\lambda & 0 \\ 0 & 1\end{array}\right) \in G L\left(2, \mathbb{H}_{\mathbb{C}}\right), \lambda \in \mathbb{R}, \lambda>0$, inversions $\left(\begin{array}{ll}0 & 1 \\ 1 & 0\end{array}\right) \in G L\left(2, \mathbb{H}_{\mathbb{C}}\right)$ and $\left(\begin{array}{ll}0 & B \\ 0 & 0\end{array}\right) \in$ $\mathfrak{g l}\left(2, \mathbb{H}_{\mathbb{C}}\right), B \in \mathbb{H}_{\mathbb{C}}$.

First, let $h=\left(\begin{array}{ll}a & 0 \\ 0 & d\end{array}\right) \in G L\left(2, \mathbb{H}_{\mathbb{C}}\right), a, d \in \mathbb{H}, N(a)=N(d)=1, \tilde{Z}=a^{-1} Z d$. Using Proposition 11 from [FL1] we obtain:

$$
\begin{array}{r}
2 \pi^{2} \cdot\left\langle\pi_{d l}(h) f, \pi_{d r}(h) g\right\rangle_{\mathcal{D R}}=\int_{Z \in S_{R}^{3}}\left(\pi_{d r}(h) g\right)(Z) \cdot(Z \otimes D z) \cdot\left((\operatorname{deg}+2)^{-1}\left(\pi_{d l}(h) f\right)\right)(Z) \\
=\int_{Z \in S_{R}^{3}} g\left(a^{-1} Z d\right) \cdot \frac{a^{-1} \otimes a^{-1}}{N(a)} \cdot(Z \otimes D z) \cdot(\operatorname{deg}+2)^{-1}\left(N(d) \cdot(d \otimes d) \cdot f\left(a^{-1} Z d\right)\right) \\
\quad=\int_{\tilde{Z} \in S_{R}^{3}} g(\tilde{Z}) \cdot(\tilde{Z} \otimes D \tilde{z}) \cdot\left((\operatorname{deg}+2)^{-1} f\right)(\tilde{Z})=2 \pi^{2} \cdot\langle f, g\rangle_{\mathcal{D R}} .
\end{array}
$$

The calculations for $h=\left(\begin{array}{ll}\lambda & 0 \\ 0 & 1\end{array}\right) \in G L\left(2, \mathbb{H}_{\mathbb{C}}\right), \lambda \in \mathbb{R}, \lambda>0$, are similar.

Next, we recall that matrices $\left(\begin{array}{ll}0 & B \\ 0 & 0\end{array}\right) \in \mathfrak{g l}\left(2, \mathbb{H}_{\mathbb{C}}\right), B \in \mathbb{H}_{\mathbb{C}}$, act by differentiation (Lemma 4 ). 
For example, if $B=\left(\begin{array}{ll}1 & 0 \\ 0 & 0\end{array}\right) \in \mathbb{H}_{\mathbb{C}}$, using the symmetric expression (27) we obtain:

$$
\begin{aligned}
& 4 \pi^{4} \cdot\left\langle\pi_{d l}\left(\begin{array}{ll}
0 & B \\
0 & 0
\end{array}\right) f, g\right\rangle_{\mathcal{D R}} \\
& =\iint_{\substack{Z \in S_{S}^{3} \\
W \in S_{R}^{3}}}\left((\operatorname{deg}+2)^{-1} g\right)(W) \cdot(W \otimes D w) \cdot k_{1}(W-Z) \cdot(Z \otimes D z) \cdot\left((\operatorname{deg}+2)^{-1} \frac{\partial f}{\partial z_{11}}\right)(Z) \\
& -\iint_{\substack{Z \in S_{R}^{3} \\
W \in S_{r}^{3}}}\left((\operatorname{deg}+2)^{-1} g\right)(W) \cdot(W \otimes D w) \cdot k_{1}(W-Z) \cdot(Z \otimes D z) \cdot\left((\operatorname{deg}+2)^{-1} \frac{\partial f}{\partial z_{11}}\right)(Z) \\
& =\iint_{\substack{Z \in S^{3} \\
W \in S_{R}^{3}}}\left((\operatorname{deg}+2)^{-1} g\right)(W) \cdot(W \otimes D w) \cdot\left(\frac{\partial}{\partial w_{11}} k_{1}(W-Z)\right) \cdot(Z \otimes D z) \cdot\left((\operatorname{deg}+2)^{-1} f\right)(Z) \\
& -\iint_{\substack{Z \in S_{R}^{3} \\
W \in S_{r}^{3}}}\left((\operatorname{deg}+2)^{-1} g\right)(W) \cdot(W \otimes D w) \cdot\left(\frac{\partial}{\partial w_{11}} k_{1}(W-Z)\right) \cdot(Z \otimes D z) \cdot\left((\operatorname{deg}+2)^{-1} f\right)(Z) \\
& =-\iint_{\substack{Z \in S_{r}^{3} \\
W \in S_{R}^{3}}}\left((\operatorname{deg}+2)^{-1} g\right)(W) \cdot(W \otimes D w) \cdot\left(\frac{\partial}{\partial z_{11}} k_{1}(W-Z)\right) \cdot(Z \otimes D z) \cdot\left((\operatorname{deg}+2)^{-1} f\right)(Z) \\
& +\iint_{\substack{Z \in S_{R}^{3} \\
W \in S_{r}^{3}}}\left((\operatorname{deg}+2)^{-1} g\right)(W) \cdot(W \otimes D w) \cdot\left(\frac{\partial}{\partial z_{11}} k_{1}(W-Z)\right) \cdot(Z \otimes D z) \cdot\left((\operatorname{deg}+2)^{-1} f\right)(Z) \\
& =-\iint_{\substack{Z \in S_{r}^{3} \\
W \in S_{R}^{3}}}\left((\operatorname{deg}+2)^{-1} \frac{\partial g}{\partial w_{11}}\right)(W) \cdot(W \otimes D w) \cdot k_{1}(W-Z) \cdot(Z \otimes D z) \cdot\left((\operatorname{deg}+2)^{-1} f\right)(Z) \\
& +\iint_{\substack{Z \in S_{R}^{3} \\
W \in S_{r}^{3}}}\left((\operatorname{deg}+2)^{-1} \frac{\partial g}{\partial w_{11}}\right)(W) \cdot(W \otimes D w) \cdot k_{1}(W-Z) \cdot(Z \otimes D z) \cdot\left((\operatorname{deg}+2)^{-1} f\right)(Z) \\
& =-4 \pi^{4} \cdot\left\langle f, \pi_{d r}\left(\begin{array}{ll}
0 & B \\
0 & 0
\end{array}\right) g\right\rangle_{\mathcal{D} \mathcal{R}} .
\end{aligned}
$$

Finally, if $h=\left(\begin{array}{ll}0 & 1 \\ 1 & 0\end{array}\right) \in G L\left(2, \mathbb{H}_{\mathbb{C}}\right)$, changing the variable to $\tilde{Z}=Z^{-1}$ - which is an orientation reversing $\operatorname{map} S_{R}^{3} \rightarrow S_{1 / R}^{3}$ - and using Proposition 11 from [FL1], we have:

$$
\begin{aligned}
& 2 \pi^{2} \cdot\left\langle\pi_{d l}(h) f, \pi_{d r}(h) g\right\rangle_{\mathcal{D R}} \\
&=\int_{Z \in S_{R}^{3}} g\left(Z^{-1}\right) \cdot \frac{Z^{-1} \otimes Z^{-1}}{N(Z)} \cdot(Z \otimes D z) \cdot(\operatorname{deg}+2)^{-1}\left(\frac{Z^{-1} \otimes Z^{-1}}{N(Z)} \cdot f\left(Z^{-1}\right)\right) \\
&=-\int_{Z \in S_{R}^{3}} g\left(Z^{-1}\right) \cdot \frac{Z^{-1} \otimes\left(Z^{-1} \cdot D z \cdot Z^{-1}\right)}{N(Z)^{2}} \cdot\left((\operatorname{deg}+2)^{-1} f\right)\left(Z^{-1}\right) \\
& \quad=-\int_{\tilde{Z} \in S_{1 / R}^{3}} g(\tilde{Z}) \cdot(\tilde{Z} \otimes D \tilde{z}) \cdot\left((\operatorname{deg}+2)^{-1} f\right)(\tilde{Z})=-2 \pi^{2} \cdot\langle f, g\rangle_{\mathcal{D R}} .
\end{aligned}
$$

(Note that the negative sign in $\left\langle\pi_{d l}(h) f, \pi_{d r}(h) g\right\rangle_{\mathcal{D} \mathcal{R}}=-\langle f, g\rangle_{\mathcal{D R}}$ does not affect the invariance of the bilinear pairing under the Lie algebra $\mathfrak{g l}\left(2, \mathbb{H}_{\mathbb{C}}\right)$.)

Next we describe orthogonality relations for doubly regular functions. Recall functions $F_{l, m, n}, F_{l, m, n}^{\prime}, G_{l, m, n}$ and $G_{l, m, n}^{\prime}$ introduced in Lemma 10, these are the functions that appear in matrix coefficient expansions of the Cauchy-Fueter kernel (23) and (24).

Proposition 20. We have the following orthogonality relations:

$$
\begin{gathered}
\left\langle F_{l, m, n}, G_{l^{\prime}, m^{\prime}, n^{\prime}}^{\prime}\right\rangle_{\mathcal{D R}}=-\left\langle F_{l, m, n}^{\prime}, G_{l^{\prime}, m^{\prime}, n^{\prime}}\right\rangle_{\mathcal{D R}}=\delta_{l l^{\prime}} \cdot \delta_{m m^{\prime}} \cdot \delta_{n n^{\prime}}, \\
\left\langle F_{l, m, n}, G_{l^{\prime}, m^{\prime}, n^{\prime}}\right\rangle_{\mathcal{D R}}=\left\langle F_{l, m, n}^{\prime}, G_{l^{\prime}, m^{\prime}, n^{\prime}}^{\prime}\right\rangle_{\mathcal{D R}}=0 .
\end{gathered}
$$


Proof. Recall that, by Lemma 6 in [FL1], the 3 -form $D z$ restricted to $S_{R}^{3}$ equals $Z d S / R$. We continue to identify tensor products of matrices with their Kronecker products. Applying Lemma 23 from [FL1] repeatedly, we compute

$$
(Z \otimes Z) \cdot F_{l, m, n}(Z)=\left(\begin{array}{c}
(l-n+1)(l-n+2) t_{n-1}^{l+1}(Z) \\
(l+n+1)(l-n+1) t_{n}^{l+1}(Z) \\
(l-n+1)(l+n+1) t_{n}^{l+1}(Z) \\
(l+n+1)(l+n+2) t_{n+1}^{l+1}(Z)
\end{array}\right) .
$$

When we multiply this by $G_{l^{\prime}, m^{\prime}, n^{\prime}}^{\prime}(Z)$ and integrate over $S_{R}^{3}$, by the orthogonality relations (17) in [FL3] (see also equation (1) in $\S 6.2$ of Chapter III in [V]),

$$
\left\langle F_{l, m, n}, G_{l^{\prime}, m^{\prime}, n^{\prime}}^{\prime}\right\rangle_{\mathcal{D R}}=\delta_{l l^{\prime}} \cdot \delta_{m m^{\prime}} \cdot \delta_{n n^{\prime}}
$$

Since

$$
G_{l^{\prime}, m^{\prime}, n^{\prime}}(Z) \cdot(Z \otimes Z) \cdot F_{l, m, n}(Z)
$$

is homogeneous of degree $2\left(l+l^{\prime}\right)>-2$ and the pairing (25) is independent of the choice of $R>0,\left\langle F_{l, m, n}, G_{l^{\prime}, m^{\prime}, n^{\prime}}\right\rangle_{\mathcal{D R}}$ must be zero. The cases involving $F_{l, m, n}^{\prime}$ can be proved similarly.

Corollary 21. The coefficients $a_{l, m, n}, b_{l, m, n}, c_{l, m, n}$ and $d_{l, m, n}$ of Laurent expansions of doubly regular functions given in Corollary 16 are given by the following expressions:

$$
\begin{array}{ll}
a_{l . m, n}=\left\langle f, G_{l, m, n}^{\prime}\right\rangle_{\mathcal{D R}}, & b_{l . m, n}=-\left\langle f, G_{l, m, n}\right\rangle_{\mathcal{D R}}, \\
c_{l . m, n}=-\left\langle F_{l, m, n}^{\prime}, g\right\rangle_{\mathcal{D R}}, & d_{l . m, n}=\left\langle F_{l, m, n}, g\right\rangle_{\mathcal{D R}} .
\end{array}
$$

\section{$2.6 n$-regular Functions}

One can generalize the notion of doubly regular functions to triply regular functions, quadruply regular functions and so on. Thus, left $n$-regular functions take values in

$$
\underbrace{\mathbb{S} \odot \cdots \odot \mathbb{S}}_{n \text { times }}
$$

and satisfy $n$ regularity conditions

$$
\left(1 \otimes \cdots \otimes \underset{i \text {-th place }}{\nabla^{+}} \otimes \cdots \otimes 1\right) f=0, \quad i=1, \ldots, n .
$$

Similarly, one can define right $n$-regular functions with values in

$$
\underbrace{\mathbb{S}^{\prime} \odot \cdots \odot \mathbb{S}^{\prime}}_{n \text { times }}
$$

The group $G L\left(2, \mathbb{H}_{\mathbb{C}}\right)$ acts on $n$-regular functions similarly to (13)-(14). Then polynomial $n$ regular functions should yield realizations of all the highest weight representations of the most degenerate series of representations of the conformal Lie algebra $\mathfrak{s l}\left(2, \mathbb{H}_{\mathbb{C}}\right)$. Those are often called the spin $\frac{n}{2}$ representations of positive and negative helicities. The spin 0 case corresponds to the harmonic functions, while the spin $\frac{1}{2}$ case correspond to the usual left and right regular functions. Such representations were considered by H. P. Jakobsen and M. Vergne in [JV1].

One can also derive an analogue of the Cauchy-Fueter formulas as well as a bilinear pairing for $n$-regular functions, just as we did for the $n=2$ case in this section. However, the $n=2$ case appears to be special, as the doubly regular functions can be realized as a certain subspace

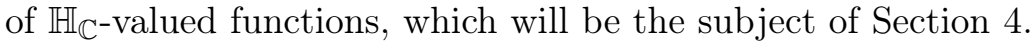


Functions with values in $\mathbb{S} \odot \cdots \odot \mathbb{S}$ are a subspace of all functions

$$
\mathbb{H}_{\mathbb{C}}^{\times} \rightarrow \underbrace{\mathbb{S} \otimes \cdots \otimes \mathbb{S}}_{n \text { times }}
$$

As a special case of Schur-Weyl duality,

$$
\underbrace{\mathbb{S} \otimes \cdots \otimes \mathbb{S}}_{n \text { times }}=\bigoplus_{k=0}^{n} M_{k} \otimes V_{k},
$$

where $V_{k}$ is the irreducible representation of $S L(2, \mathbb{C})$ of dimension $k+1$ and $M_{k}$ is its multiplicity, which is also an irreducible representation of the symmetric group on $n$ objects. In particular,

$$
V_{n}=\underbrace{\mathbb{S} \odot \cdots \odot \mathbb{S}}_{n \text { times }} \quad \text { and } \quad V_{0}=\underbrace{\mathbb{S} \wedge \cdots \wedge \mathbb{S}}_{n \text { times }} \text {. }
$$

When $n=2, M_{1}=0$ and

$$
\mathbb{S} \otimes \mathbb{S}=(\mathbb{S} \wedge \mathbb{S}) \oplus(\mathbb{S} \odot \mathbb{S})
$$

Proposition 22. As a representation of $\mathfrak{g l}\left(2, \mathbb{H}_{\mathbb{C}}\right)$, the space of maps

$$
\mathbb{H}_{\mathbb{C}}^{\times} \rightarrow \mathbb{S} \wedge \mathbb{S}
$$

is isomorphic to $\mathbb{C}\left[z_{11}, z_{12}, z_{21}, z_{22}, N(Z)^{-1}\right]$ with $\mathfrak{g l}\left(2, \mathbb{H}_{\mathbb{C}}\right)$ action obtained by differentiating the following action of $G L\left(2, \mathbb{H}_{\mathbb{C}}\right)$ :

$$
f(Z) \mapsto \frac{1}{N(c Z+d)^{2}} \cdot f\left((a Z+b)(c Z+d)^{-1}\right), \quad h^{-1}=\left(\begin{array}{ll}
a & b \\
c & d
\end{array}\right) \in G L\left(2, \mathbb{H}_{\mathbb{C}}\right) .
$$

Similarly, as a representation of $\mathfrak{g l}\left(2, \mathbb{H}_{\mathbb{C}}\right)$, the space of maps

$$
\mathbb{H}_{\mathbb{C}}^{\times} \rightarrow \mathbb{S}^{\prime} \wedge \mathbb{S}^{\prime}
$$

is isomorphic to $\mathbb{C}\left[z_{11}, z_{12}, z_{21}, z_{22}, N(Z)^{-1}\right]$ with $\mathfrak{g l}\left(2, \mathbb{H}_{\mathbb{C}}\right)$ action obtained by differentiating another action of $G L\left(2, \mathbb{H}_{\mathbb{C}}\right)$ :

$$
f(Z) \mapsto \frac{1}{N\left(a^{\prime}-Z c^{\prime}\right)^{2}} \cdot f\left(\left(a^{\prime}-Z c^{\prime}\right)^{-1}\left(-b^{\prime}+Z d^{\prime}\right)\right), \quad h=\left(\begin{array}{cc}
a^{\prime} & b^{\prime} \\
c^{\prime} & d^{\prime}
\end{array}\right) \in G L\left(2, \mathbb{H}_{\mathbb{C}}\right) .
$$

Proof. Since $\mathbb{S} \wedge \mathbb{S}$ and $\mathbb{S}^{\prime} \wedge \mathbb{S}^{\prime}$ are one-dimensional, the spaces of functions $\mathbb{H}_{\mathbb{C}}^{\times} \rightarrow \mathbb{S} \wedge \mathbb{S}$ and $\mathbb{H}_{\mathbb{C}}^{\times} \rightarrow \mathbb{S}^{\prime} \wedge \mathbb{S}^{\prime}$ can be both identified with $\mathbb{C}\left[z_{11}, z_{12}, z_{21}, z_{22}, N(Z)^{-1}\right]$. The actions of $\mathfrak{g l}\left(2, \mathbb{H}_{\mathbb{C}}\right)$ are obtained by taking the determinants of $\pi_{d l}$ and $\pi_{d r}$ respectively, and the result follows from (13) $-(14)$.

Note that similar representations and their irreducible components were considered in Subsections 3.1-3.2 in [L1].

For general $n$, we have a decomposition of the space of functions $\mathbb{H}_{\mathbb{C}}^{\times} \rightarrow \mathbb{S} \otimes \cdots \otimes \mathbb{S}$ according to (28). In a separate paper [FL4] we study $n$-regular functions in more detail. For example, we prove that generalizations of the Cauchy-Fueter formulas to such functions provide natural quaternionic analogues of Cauchy's differentiation formula

$$
f^{(n-1)}(w)=\frac{(n-1) !}{2 \pi i} \oint \frac{f(z) d z}{(z-w)^{n}} .
$$




\section{Quaternionic Chain Complex and Decomposition of $(\rho, \mathbf{W})$, $\left(\rho^{\prime}, \mathbf{W}^{\prime}\right)$ into Irreducible Components}

\subsection{Quaternionic Chain Complex}

We start with a sequence of maps (57) from [FL1]:

$$
\left(\rho^{\prime}, H^{\prime}\right) \stackrel{\partial^{+}}{\longrightarrow}\left(\rho_{2}^{\prime}, \mathcal{W}^{\prime}\right) \stackrel{\mathrm{Mx}}{\longrightarrow}\left(\rho_{2}, \mathcal{W}\right) \stackrel{\operatorname{Tr} \circ \partial^{+}}{\longrightarrow}(\rho, \amalg)
$$

where

$$
\begin{aligned}
& \amalg=H^{\prime}=\left\{\mathbb{C} \text {-valued polynomial functions on } \mathbb{H}_{\mathbb{C}}^{\times}\right\}=\mathbb{C}\left[z_{11}, z_{12}, z_{21}, z_{22}, N(Z)^{-1}\right],
\end{aligned}
$$

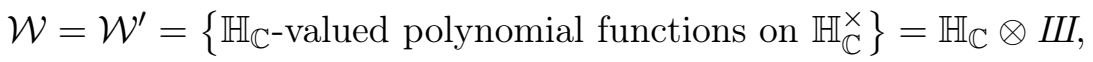

$$
\begin{aligned}
& \partial=\left(\begin{array}{ll}
\partial_{11} & \partial_{21} \\
\partial_{12} & \partial_{22}
\end{array}\right)=\frac{1}{2} \nabla, \quad \partial^{+}=\left(\begin{array}{cc}
\partial_{22} & -\partial_{21} \\
-\partial_{12} & \partial_{11}
\end{array}\right)=\frac{1}{2} \nabla^{+}, \quad \text { Mx } F=\nabla F \nabla-\square F^{+} .
\end{aligned}
$$

Since the compositions of any two consecutive maps are zero:

$$
\mathrm{Mx} \circ \partial^{+}=0 \quad \text { and } \quad\left(\operatorname{Tr} \circ \partial^{+}\right) \circ \mathrm{Mx}=0,
$$

we call (29) a quaternionic chain complex. The Lie algebra $\mathfrak{g l}\left(2, \mathbb{H}_{\mathbb{C}}\right)$ acts on these spaces by differentiating the following group actions:

$$
\begin{array}{rlll}
\rho(h): f(Z) & \mapsto & (\rho(h) f)(Z)=\frac{f\left((a Z+b)(c Z+d)^{-1}\right)}{N(c Z+d)^{2} \cdot N\left(a^{\prime}-Z c^{\prime}\right)^{2}}, \\
\rho^{\prime}(h): f(Z) & \mapsto & (\rho(h) f)(Z)=f\left((a Z+b)(c Z+d)^{-1}\right), \\
\rho_{2}(h): F(Z) & \mapsto & \left(\rho_{2}(h) F\right)(Z)=\frac{(c Z+d)^{-1}}{N(c Z+d)} \cdot F\left((a Z+b)(c Z+d)^{-1}\right) \cdot \frac{\left(a^{\prime}-Z c^{\prime}\right)^{-1}}{N\left(a^{\prime}-Z c^{\prime}\right)}, \\
\rho_{2}^{\prime}(h): F(Z) & \mapsto & \left(\rho_{2}^{\prime}(h) F\right)(Z)=\frac{\left(a^{\prime}-Z c^{\prime}\right)}{N\left(a^{\prime}-Z c^{\prime}\right)} \cdot F\left((a Z+b)(c Z+d)^{-1}\right) \cdot \frac{(c Z+d)}{N(c Z+d)},
\end{array}
$$

where $f \in W$ or $H^{\prime}, F \in \mathcal{W}$ or $\mathcal{W}^{\prime}, h=\left(\begin{array}{cc}a^{\prime} & b^{\prime} \\ c^{\prime} & d^{\prime}\end{array}\right) \in G L\left(2, \mathbb{H}_{\mathbb{C}}\right)$ and $h^{-1}=\left(\begin{array}{ll}a & b \\ c & d\end{array}\right)$. Although $\amalg=W^{\prime}$ and $\mathcal{W}=\mathcal{W}^{\prime}$ as vector spaces, these notations indicate the action of $\mathfrak{g l}\left(2, \mathbb{H}_{\mathbb{C}}\right)$. Also, in [FL3] we treat $\left(\rho_{1}, \mathscr{W}\right)$, where $\mathscr{T}=W$ as vector spaces, but the action $\rho_{1}$ of $\mathfrak{g l}\left(2, \mathbb{H}_{\mathbb{C}}\right)$ is different from $\rho, \rho^{\prime}$ considered here. We have the following four analogues of Lemma 68 in [FL1]:

Lemma 23. The Lie algebra action $\rho$ of $\mathfrak{g l}\left(2, \mathbb{H}_{\mathbb{C}}\right)$ on $\amalg$ is given by

$$
\begin{aligned}
& \rho\left(\begin{array}{ll}
A & 0 \\
0 & 0
\end{array}\right): f(Z) \mapsto-\operatorname{Tr}(A Z \partial+2 A) f, \\
& \rho\left(\begin{array}{ll}
0 & B \\
0 & 0
\end{array}\right): f(Z) \mapsto-\operatorname{Tr}(B \partial) f, \\
& \rho\left(\begin{array}{cc}
0 & 0 \\
C & 0
\end{array}\right): f(Z) \mapsto \operatorname{Tr}(Z C Z \partial+4 C Z) f, \\
& \rho\left(\begin{array}{ll}
0 & 0 \\
0 & D
\end{array}\right): f(Z) \mapsto \operatorname{Tr}(Z D \partial+2 D) f .
\end{aligned}
$$

Lemma 24. The Lie algebra action $\rho^{\prime}$ of $\mathfrak{g l}\left(2, \mathbb{H}_{\mathbb{C}}\right)$ on $W^{\prime}$ is given by

$$
\begin{aligned}
& \rho^{\prime}\left(\begin{array}{ll}
A & 0 \\
0 & 0
\end{array}\right): f(Z) \mapsto-\operatorname{Tr}(A Z \partial) f, \\
& \rho^{\prime}\left(\begin{array}{ll}
0 & B \\
0 & 0
\end{array}\right): f(Z) \mapsto-\operatorname{Tr}(B \partial) f, \\
& \rho^{\prime}\left(\begin{array}{ll}
0 & 0 \\
C & 0
\end{array}\right): f(Z) \mapsto \operatorname{Tr}(Z C Z \partial) f, \\
& \rho^{\prime}\left(\begin{array}{ll}
0 & 0 \\
0 & D
\end{array}\right): f(Z) \mapsto \operatorname{Tr}(Z D \partial) f .
\end{aligned}
$$


Lemma 25. The Lie algebra action $\rho_{2}$ of $\mathfrak{g l}\left(2, \mathbb{H}_{\mathbb{C}}\right)$ on $\mathcal{W}$ is given by

$$
\begin{aligned}
& \rho_{2}\left(\begin{array}{ll}
A & 0 \\
0 & 0
\end{array}\right): F(Z) \mapsto-\operatorname{Tr}(A Z \partial+A) F-F A, \\
& \rho_{2}\left(\begin{array}{ll}
0 & B \\
0 & 0
\end{array}\right): F(Z) \mapsto-\operatorname{Tr}(B \partial) F, \\
& \rho_{2}\left(\begin{array}{ll}
0 & 0 \\
C & 0
\end{array}\right): F(Z) \mapsto \operatorname{Tr}(Z C Z \partial+2 Z C) F+C Z F+F Z C, \\
& \rho_{2}\left(\begin{array}{ll}
0 & 0 \\
0 & D
\end{array}\right): F(Z) \mapsto \operatorname{Tr}(Z D \partial+D) F+D F .
\end{aligned}
$$

Lemma 26. The Lie algebra action $\rho_{2}^{\prime}$ of $\mathfrak{g l}\left(2, \mathbb{H}_{\mathbb{C}}\right)$ on $\mathcal{W}^{\prime}$ is given by

$$
\begin{aligned}
& \rho_{2}^{\prime}\left(\begin{array}{ll}
A & 0 \\
0 & 0
\end{array}\right): F(Z) \mapsto-\operatorname{Tr}(A Z \partial+A) F+A F, \\
& \rho_{2}^{\prime}\left(\begin{array}{ll}
0 & B \\
0 & 0
\end{array}\right): F(Z) \mapsto-\operatorname{Tr}(B \partial) F, \\
& \rho_{2}^{\prime}\left(\begin{array}{ll}
0 & 0 \\
C & 0
\end{array}\right): F(Z) \mapsto \operatorname{Tr}(Z C Z \partial+2 Z C) F-Z C F-F C Z, \\
& \rho_{2}^{\prime}\left(\begin{array}{ll}
0 & 0 \\
0 & D
\end{array}\right): F(Z) \mapsto \operatorname{Tr}(Z D \partial+D) F-F D .
\end{aligned}
$$

Next, we show that the maps in the quaternionic chain complex (29) are $\mathfrak{g l}\left(2, \mathbb{H}_{\mathbb{C}}\right)$-equivariant.

Proposition 27. The map $\partial^{+}:\left(\rho^{\prime}, H^{\prime}\right) \rightarrow\left(\rho_{2}^{\prime}, \mathcal{W}^{\prime}\right)$ in (29) is $\mathfrak{g l}\left(2, \mathbb{H}_{\mathbb{C}}\right)$-equivariant.

Proof. For $f(Z) \in H^{\prime}$, by direct computation we obtain:

$$
\begin{gathered}
-\partial^{+} \operatorname{Tr}(A Z \partial) f=-\operatorname{Tr}(A Z \partial) \partial^{+} f+\left(\begin{array}{rr}
-a_{12} \partial_{12}-a_{22} \partial_{22} & a_{12} \partial_{11}+a_{22} \partial_{21} \\
a_{11} \partial_{12}+a_{21} \partial_{22} & -a_{11} \partial_{11}-a_{21} \partial_{21}
\end{array}\right) f \\
=-\operatorname{Tr}(A Z \partial) \partial^{+} f-\operatorname{Tr}(A) \partial^{+} f+A \partial^{+} f \\
\partial^{+} \operatorname{Tr}(Z C Z \partial) f=\operatorname{Tr}(Z C Z \partial) \partial^{+} f \\
+\left(\begin{array}{cc}
c_{21} z_{11} \partial_{21}+c_{22} z_{21} \partial_{21}+c_{21} z_{12} \partial_{22}+2 c_{22} z_{22} \partial_{22} & -c_{11} z_{11} \partial_{21}-2 c_{12} z_{21} \partial_{21}-c_{11} z_{12} \partial_{22}-c_{12} z_{22} \partial_{22} \\
+c_{12} z_{11} \partial_{12}+c_{12} z_{21} \partial_{22}+c_{22} z_{12} \partial_{12} & -c_{12} z_{11} \partial_{11}-c_{22} z_{12} \partial_{11}-c_{22} z_{22} \partial_{21} \\
-c_{21} z_{11} \partial_{11}-c_{22} z_{21} \partial_{11}-2 c_{21} z_{12} \partial_{12}-c_{22} z_{22} \partial_{12} & 2 c_{11} z_{11} \partial_{11}+c_{12} z_{21} \partial_{11}+c_{11} z_{12} \partial_{12}+c_{12} z_{22} \partial_{12} \\
-c_{11} z_{11} \partial_{12}-c_{11} z_{21} \partial_{22}-c_{21} z_{22} \partial_{22} & +c_{11} z_{21} \partial_{21}+c_{21} z_{12} \partial_{11}+c_{21} z_{22} \partial_{21}
\end{array}\right) f \\
=\operatorname{Tr}(Z C Z \partial) \partial^{+} f+2 \operatorname{Tr}(Z C) \partial^{+} f-Z C \partial^{+} f-\left(\partial^{+} f\right) C Z .
\end{gathered}
$$

The calculations showing that $\partial^{+}$intertwines the actions of $\left(\begin{array}{ll}0 & B \\ 0 & 0\end{array}\right)$ and $\left(\begin{array}{ll}0 & 0 \\ 0 & D\end{array}\right)$ are similar.

Proposition 28. The map $\mathrm{Mx}:\left(\rho_{2}^{\prime}, \mathcal{W}^{\prime}\right) \rightarrow\left(\rho_{2}, \mathcal{W}\right)$ in (29) is $\mathfrak{g l}\left(2, \mathbb{H}_{\mathbb{C}}\right)$-equivariant.

Proof. Note that $\mathrm{Mx} F=4\left(\partial F \partial-\partial \partial^{+} F^{+}\right)$, where $F(Z) \in \mathcal{W}^{\prime}$. Using

$$
\partial[\operatorname{Tr}(A Z \partial) F]=\operatorname{Tr}(A Z \partial)(\partial F)+\partial A F, \quad \partial^{+}[\operatorname{Tr}(A Z \partial) F]=\operatorname{Tr}(A Z \partial)\left(\partial^{+} F\right)+A^{+} \partial^{+} F,
$$

we obtain

$$
\begin{gathered}
\begin{array}{c}
\frac{1}{4} \operatorname{Mx}(-\operatorname{Tr}(A Z \partial+A) F+A F) \\
=-[\operatorname{Tr}(A Z \partial)(\partial F)] \overleftarrow{\partial}+\partial\left[\operatorname{Tr}(A Z \partial)\left(\partial^{+} F^{+}\right)+A^{+} \partial^{+} F^{+}\right]+\operatorname{Tr}(A) \partial \partial^{+} F^{+}-\partial \partial^{+} F^{+} A^{+} \\
=-\operatorname{Tr}(A Z \partial+A)(\partial F \partial)-(\partial F \partial) A+\operatorname{Tr}(A Z \partial)\left(\partial \partial^{+} F^{+}\right)+\partial A \partial^{+} F^{+}+\partial A^{+} \partial^{+} F^{+}+\partial \partial^{+} F^{+} A \\
=-\operatorname{Tr}(A Z \partial+A)\left(\partial F \partial-\partial \partial^{+} F^{+}\right)-\left(\partial F \partial-\partial \partial^{+} F^{+}\right) A ; \\
-\operatorname{Mx} \operatorname{Tr}(B \partial) F=-\operatorname{Tr}(B \partial) \operatorname{Mx} F ;
\end{array}
\end{gathered}
$$

similarly, using

$$
\partial[\operatorname{Tr}(Z D \partial) F]=\operatorname{Tr}(Z D \partial)(\partial F)+D \partial F, \quad \partial^{+}[\operatorname{Tr}(Z D \partial) F]=\operatorname{Tr}(Z D \partial)\left(\partial^{+} F\right)+\partial^{+} D^{+} F,
$$


we obtain

$$
\begin{array}{r}
\frac{1}{4} \operatorname{Mx}(\operatorname{Tr}(Z D \partial+D) F-F D)=[\operatorname{Tr}(Z D \partial+D)(\partial F)+D \partial F-\partial F D] \overleftarrow{\partial} \\
-\partial\left[\operatorname{Tr}(Z D \partial)\left(\partial^{+} F^{+}\right)+\partial^{+} D^{+} F^{+}\right]-\operatorname{Tr}(D) \partial \partial^{+} F^{+}+D^{+} \partial \partial^{+} F^{+} \\
=\operatorname{Tr}(Z D \partial+D)(\partial F \partial)+D(\partial F \partial)-\operatorname{Tr}(Z D \partial)\left(\partial \partial^{+} F^{+}\right)-D \partial \partial^{+} F^{+}-\operatorname{Tr}(D) \partial \partial^{+} F^{+} \\
=\operatorname{Tr}(Z D \partial+D)\left(\partial F \partial-\partial \partial^{+} F^{+}\right)+D\left(\partial F \partial-\partial \partial^{+} F^{+}\right)
\end{array}
$$

finally, using

$$
\begin{aligned}
\partial[\operatorname{Tr}(Z C Z \partial+2 Z C) F-Z C F-F C Z] & \\
& =\operatorname{Tr}(Z C Z \partial+2 Z C)(\partial F)+C Z(\partial F)-(\partial F) C Z-\operatorname{Tr}(F C),
\end{aligned}
$$

we obtain

$$
\begin{gathered}
\frac{1}{4} \operatorname{Mx}(\operatorname{Tr}(Z C Z \partial+2 Z C) F-Z C F-F C Z)=\operatorname{Tr}(Z C Z \partial+2 Z C)(\partial F \partial)+C Z(\partial F \partial)+(\partial F \partial) Z C \\
+C \operatorname{Tr}(\partial F)-\partial \operatorname{Tr}(F C)-\left(\partial^{+}[\operatorname{Tr}(Z C Z \partial+2 Z C)(\partial F)+C Z \partial F-(\partial F) C Z-\operatorname{Tr}(F C)]\right)^{+} \\
=\operatorname{Tr}(Z C Z \partial+2 Z C)(\partial F \partial)+C Z(\partial F \partial)+(\partial F \partial) Z C+C \operatorname{Tr}(\partial F)-\partial \operatorname{Tr}(F C) \\
-\operatorname{Tr}(Z C Z \partial+2 Z C)\left(\partial^{+} \partial F^{+}\right)-\left(\partial^{+} \partial F^{+}\right) Z C-C Z\left(\partial^{+} \partial F^{+}\right)-F^{+} \partial^{+} C-\partial F C+\partial \operatorname{Tr}(F C) \\
=\operatorname{Tr}(Z C Z \partial+2 Z C)\left(\partial F \partial-\partial \partial^{+} F^{+}\right)+C Z\left(\partial F \partial-\partial \partial^{+} F^{+}\right)+\left(\partial F \partial-\partial \partial^{+} F^{+}\right) Z C .
\end{gathered}
$$

Proposition 29. The map $\operatorname{Tr} \circ \partial^{+}:\left(\rho_{2}, \mathcal{W}\right) \rightarrow(\rho, W)$ in (29) is $\mathfrak{g l}\left(2, \mathbb{H}_{\mathbb{C}}\right)$-equivariant.

Proof. For $F(Z) \in \mathcal{W}$, by direct computation we obtain:

$$
\begin{gathered}
-\operatorname{Tr}\left(\partial^{+} \operatorname{Tr}(A Z \partial) F+\partial^{+} \operatorname{Tr}(A) F+\left(\partial^{+} F\right) A\right)=-\operatorname{Tr}(A Z \partial) \operatorname{Tr}\left(\partial^{+} F\right)-(\operatorname{Tr} A) \operatorname{Tr}\left(\partial^{+} F\right) \\
-\operatorname{Tr}\left(A \partial^{+} F\right)-\operatorname{Tr}\left[\left(\begin{array}{cc}
a_{12} \partial_{12}+a_{22} \partial_{22} & -a_{12} \partial_{11}-a_{22} \partial_{21} \\
-a_{11} \partial_{12}-a_{21} \partial_{22} & a_{11} \partial_{11}+a_{21} \partial_{21}
\end{array}\right) F\right]=-\operatorname{Tr}(A Z \partial+2 A) \operatorname{Tr}\left(\partial^{+} F\right) \\
\operatorname{Tr}\left(\partial^{+} \operatorname{Tr}(Z C Z \partial+2 Z C) F+\partial^{+} C Z F+\partial^{+} F Z C\right) \\
=\operatorname{Tr}(Z C Z \partial+2 Z C) \operatorname{Tr}\left(\partial^{+} F\right)+\operatorname{Tr}\left(C Z\left(F \partial^{+}\right)+Z C \partial^{+} F\right) \\
+\operatorname{Tr}\left[\left(\begin{array}{rr}
c_{21} z_{11} \partial_{21}+c_{22} z_{21} \partial_{21}+c_{21} z_{12} \partial_{22}+2 c_{22} z_{22} \partial_{22} & -c_{11} z_{11} \partial_{21}-2 c_{12} z_{21} \partial_{21}-c_{11} z_{12} \partial_{22}-c_{12} z_{22} \partial_{22} \\
+c_{12} z_{11} \partial_{12}+c_{12} z_{21} \partial_{22}+c_{22} z_{12} \partial_{12} & -c_{12} z_{11} \partial_{11}-c_{22} z_{12} \partial_{11}-c_{22} z_{22} \partial_{21} \\
-c_{21} z_{11} \partial_{11}-c_{22} z_{21} \partial_{11}-2 c_{21} z_{12} \partial_{12}-c_{22} z_{22} \partial_{12} & 2 c_{11} z_{11} \partial_{11}+c_{12} z_{21} \partial_{11}+c_{11} z_{12} \partial_{12}+c_{12} z_{22} \partial_{12} \\
-c_{11} z_{11} \partial_{12}-c_{11} z_{21} \partial_{22}-c_{21} z_{22} \partial_{22} & +c_{11} z_{21} \partial_{21}+c_{21} z_{12} \partial_{11}+c_{21} z_{22} \partial_{21}
\end{array}\right] F\right. \\
=\operatorname{Tr}(Z C Z \partial+4 Z C) \operatorname{Tr}\left(\partial^{+} F\right) .
\end{gathered}
$$

The calculations showing that $\operatorname{Tr} \circ \partial^{+}$intertwines the actions of $\left(\begin{array}{ll}0 & B \\ 0 & 0\end{array}\right)$ and $\left(\begin{array}{ll}0 & 0 \\ 0 & D\end{array}\right)$ are similar.

We have another equivariant map that does not appear in (29):

Proposition 30. The map $\square \circ \square:\left(\rho^{\prime}, I I^{\prime}\right) \rightarrow(\rho, I I)$ is $\mathfrak{g l}\left(2, \mathbb{H}_{\mathbb{C}}\right)$-equivariant.

Proof. Note that $\square=4 \partial \partial^{+}=4 \partial^{+} \partial$. For $f(Z) \in H^{\prime}$, using (30) and (31), we obtain:

$$
-\frac{1}{4} \square[\operatorname{Tr}(A Z \partial) f]=-\partial\left[\operatorname{Tr}(A Z \partial)\left(\partial^{+} f\right)-A^{+} \partial^{+} f\right]=-\operatorname{Tr}(A Z \partial)\left(\partial \partial^{+} f\right)-\operatorname{Tr}(A) \partial \partial^{+} f,
$$




$$
\begin{aligned}
& -(\square \circ \square)[\operatorname{Tr}(A Z \partial) f]=-\operatorname{Tr}(A Z \partial+2 A)(\square \circ \square f) ; \\
& \frac{1}{4} \square[\operatorname{Tr}(Z C Z \partial) f]=\partial\left[\operatorname{Tr}(Z C Z \partial)\left(\partial^{+} f\right)+\left(\partial^{+} f\right) Z^{+} C^{+}+C^{+} Z^{+} \partial^{+} f\right] \\
& =\operatorname{Tr}(Z C Z \partial)\left(\partial \partial^{+} f\right)+C Z\left(\partial \partial^{+} f\right)+\partial\left[Z C \partial^{+} f\right]-2 C \partial^{+} f+\partial\left[\left(\partial^{+} f\right) Z^{+} C^{+}+C^{+} Z^{+} \partial^{+} f\right] \\
& =\operatorname{Tr}(Z C Z \partial)\left(\partial \partial^{+} f\right)+\left(C Z+Z^{+} C^{+}\right)\left(\partial \partial^{+} f\right)+\partial\left[Z C \partial^{+} f+C^{+} Z^{+} \partial^{+} f\right]-2 C \partial^{+} f-(\partial f) C^{+} \\
& =\operatorname{Tr}(Z C Z \partial+2 C Z)\left(\partial \partial^{+} f\right)-\operatorname{Tr}\left(C^{+} \partial\right) f \\
& (\square \circ \square)[\operatorname{Tr}(Z C Z \partial) f]=\operatorname{Tr}(Z C Z \partial+4 C Z)(\square \circ \square f) .
\end{aligned}
$$

The calculations showing that $\square \circ \square$ intertwines the actions of $\left(\begin{array}{ll}0 & B \\ 0 & 0\end{array}\right)$ and $\left(\begin{array}{ll}0 & 0 \\ 0 & D\end{array}\right)$ are similar.

\subsection{Decomposition of $(\rho, \amalg)$ and $\left(\rho^{\prime}, \mathbf{W}^{\prime}\right)$ into Irreducible Components}

Similarly to how we proved Theorem 8 in [L1], we can obtain the following two decomposition results.

Theorem 31. The only proper $\mathfrak{g l}\left(2, \mathbb{H}_{\mathbb{C}}\right)$-invariant subspaces of $(\rho, W)$ are

$$
\begin{aligned}
H^{+} & =\mathbb{C} \text {-span of }\left\{N(Z)^{k} \cdot t_{n \underline{m}}^{l}(Z) ; k \geq 0\right\}, \\
I^{-} & =\mathbb{C} \text {-span of }\left\{N(Z)^{k} \cdot t_{n \underline{m}}^{l}(Z) ; k \leq-(2 l+4)\right\}, \\
\mathcal{I}^{+} & =\mathbb{C} \text {-span of }\left\{N(Z)^{k} \cdot t_{n \underline{m}}^{l}(Z) ; k \geq-(2 l+1)\right\}, \\
\mathcal{I}^{-} & =\mathbb{C} \text {-span of }\left\{N(Z)^{k} \cdot t_{n \underline{m}}^{l}(Z) ; k \leq-3\right\}, \\
\mathcal{J} & =\mathbb{C} \text {-span of }\left\{N(Z)^{k} \cdot t_{n \underline{m}}^{l}(Z) ;-(2 l+1) \leq k \leq-3\right\}
\end{aligned}
$$

and their sums (see Figure 2).

The irreducible components of $(\rho, W)$ are the subrepresentations

$$
\left(\rho, H^{+}\right), \quad\left(\rho, \Pi^{-}\right), \quad(\rho, \mathcal{J})
$$

and the quotients

$$
\left(\rho, \mathcal{I}^{+} /\left(H^{+} \oplus \mathcal{J}\right)\right), \quad\left(\rho, \mathcal{I}^{-} /\left(H^{-} \oplus \mathcal{J}\right)\right), \quad\left(\rho, W /\left(\mathcal{I}^{+}+\mathcal{I}^{-}\right)\right)
$$

(see Figure 3).

Theorem 32. The only proper $\mathfrak{g l}\left(2, \mathbb{H}_{\mathbb{C}}\right)$-invariant subspaces of $\left(\rho^{\prime}, \amalg^{\prime}\right)$ are

$$
\begin{aligned}
\mathcal{I}_{0}^{\prime} & =\mathbb{C}=\mathbb{C} \text {-span of }\left\{N(Z)^{0} \cdot t_{0 \underline{0}}^{0}(Z)\right\}, \\
\mathcal{B} \mathcal{H}^{+} & =\mathbb{C} \text {-span of }\left\{N(Z)^{k} \cdot t_{n \underline{m}}^{l}(Z) ; 0 \leq k \leq 1\right\}, \\
\mathcal{B H} \mathcal{H}^{-} & =\mathbb{C} \text {-span of }\left\{N(Z)^{k} \cdot t_{n \underline{m}}^{l}(Z) ;-1 \leq 2 l+k \leq 0\right\}, \\
H^{+} & =\mathbb{C} \text {-span of }\left\{N(Z)^{k} \cdot t_{n \underline{m}}^{l}(Z) ; k \geq 0\right\}, \\
H^{\prime-} & =\mathbb{C} \text {-span of }\left\{N(Z)^{k} \cdot t_{n \underline{m}}^{l}(Z) ; k \leq-2 l\right\}, \\
\mathcal{I}^{\prime+} & =\mathbb{C} \text {-span of }\left\{N(Z)^{k} \cdot t_{n \underline{m}}^{l}(Z) ; k \geq-(2 l+1)\right\}, \\
\mathcal{I}^{\prime-} & =\mathbb{C} \text {-span of }\left\{N(Z)^{k} \cdot t_{n \underline{m}}^{l}(Z) ; k \leq 1\right\}, \\
\mathcal{J}^{\prime} & =\mathbb{C} \text {-span of }\left\{N(Z)^{k} \cdot t_{n \underline{m}}^{l}(Z) ;-(2 l+1) \leq k \leq 1\right\}
\end{aligned}
$$

and their sums (see Figure 4). 


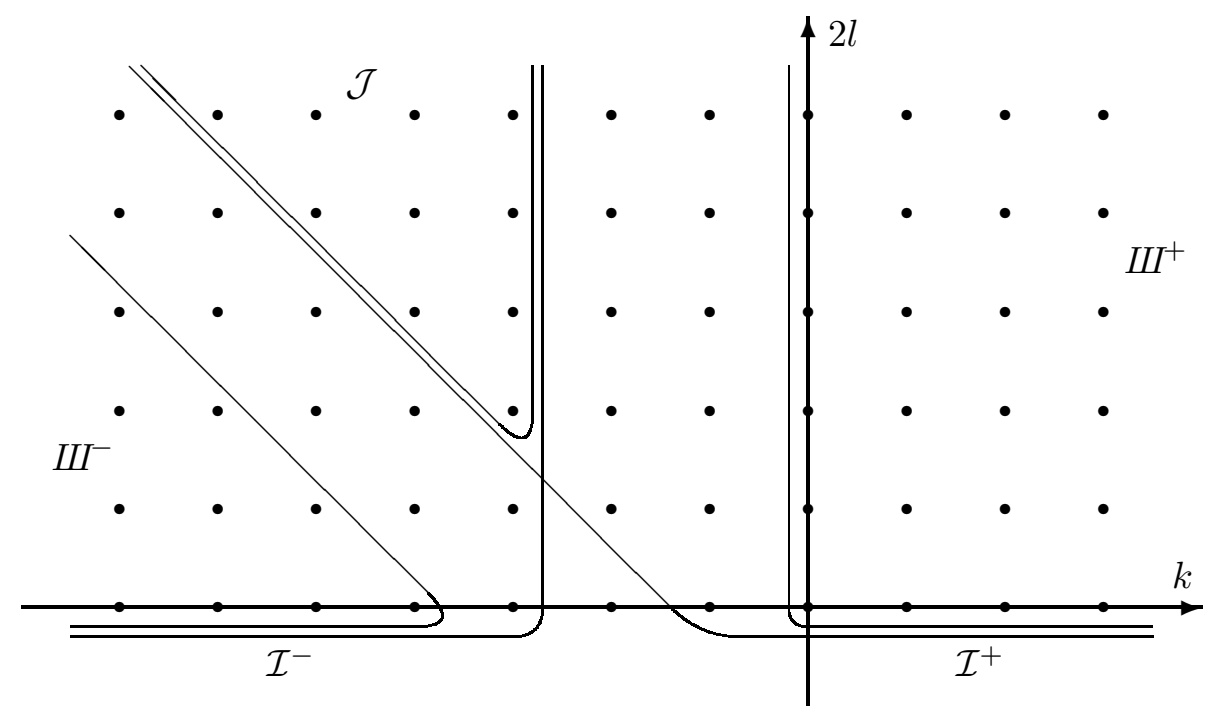

Figure 2: Decomposition of $(\rho, W)$ into irreducible components.

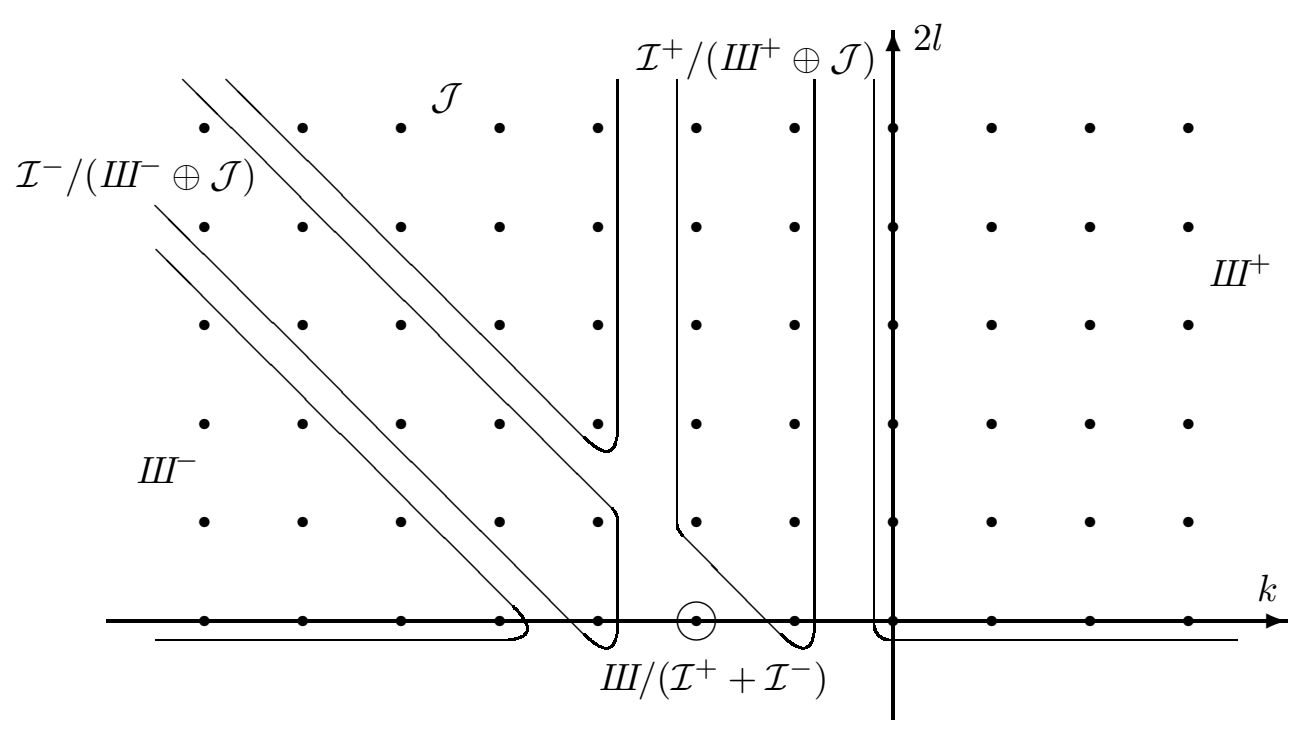

Figure 3: Irreducible components of $(\rho, I I)$. 


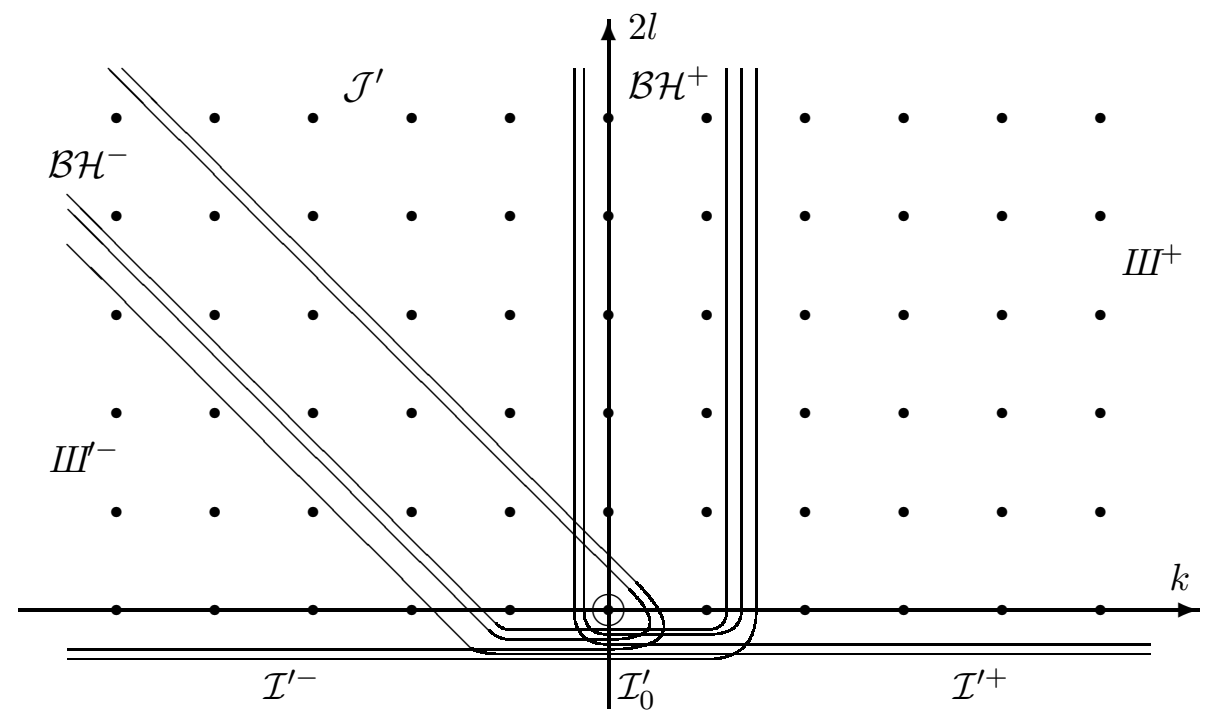

Figure 4: Decomposition of $\left(\rho^{\prime}, I I^{\prime}\right)$ into irreducible components.

The irreducible components of $\left(\rho^{\prime}, I^{\prime}\right)$ are the trivial subrepresentation $\left(\rho^{\prime}, \mathcal{I}_{0}^{\prime}\right)$ and the quotients

$$
\begin{aligned}
& \left(\rho^{\prime}, \mathcal{B H}^{+} / \mathcal{I}_{0}^{\prime}\right), \quad\left(\rho^{\prime}, \mathcal{B H}^{-} / \mathcal{I}_{0}^{\prime}\right), \\
& \left(\rho^{\prime}, W^{+} / \mathcal{B H}^{+}\right)=\left(\rho^{\prime}, W / \mathcal{I}^{\prime-}\right), \quad\left(\rho^{\prime}, W^{\prime-} / \mathcal{B H}^{-}\right)=\left(\rho^{\prime}, W / \mathcal{I}^{\prime+}\right), \\
& \left(\rho^{\prime}, \amalg /\left(\mathcal{I}^{\prime+}+\mathcal{I}^{\prime-}\right)\right)=\left(\rho^{\prime}, \mathcal{I}^{\prime+} /\left(W^{+}+\mathcal{B H} \mathcal{H}^{-}\right)\right)=\left(\rho^{\prime}, \mathcal{I}^{\prime-} /\left(W^{\prime-}+\mathcal{B H}^{+}\right)\right)=\left(\rho^{\prime}, \mathcal{J}^{\prime} /\left(\mathcal{B H}^{+}+\mathcal{B H}^{-}\right)\right)
\end{aligned}
$$

(see Figure [5, which is essentially a shifted Figure 3).

Corollary 33. The image under the $\mathfrak{g l}\left(2, \mathbb{H}_{\mathbb{C}}\right)$-equivariant map $\square \circ \square:\left(\rho^{\prime}, H^{\prime}\right) \rightarrow(\rho, H)$ from Proposition 30 is $\mathrm{WH}^{+} \oplus \mathcal{J} \oplus \mathrm{WI}^{-}$; this map provides isomorphisms

$$
\left(\rho^{\prime}, H^{+} / \mathcal{B} \mathcal{H}^{+}\right) \simeq\left(\rho, H^{+}\right), \quad\left(\rho^{\prime}, \mathcal{J}^{\prime} /\left(\mathcal{B} \mathcal{H}^{+}+\mathcal{B} \mathcal{H}^{-}\right)\right) \simeq(\rho, \mathcal{J}), \quad\left(\rho^{\prime}, H^{\prime-} / \mathcal{B H} \mathcal{H}^{-}\right) \simeq\left(\rho, H^{-}\right) .
$$

Proof. The result follows from Theorems 31, 32 and an identity:

$$
\square\left(N(Z)^{k} \cdot t_{n \underline{m}}^{l}(Z)\right)=4 k(2 l+k+1) N(Z)^{k-1} \cdot t_{n \underline{m}}^{l}(Z),
$$

which can be verified by direct computation.

We call a function $f$ biharmonic if $(\square \circ \square) f=0$. Using (33), we can characterize the space of biharmonic functions.

Proposition 34. We have:

$$
\{f \in H ;(\square \circ \square) f=0\}=\mathcal{B H}^{+}+\mathcal{B H}^{-} .
$$

In other words, a function $f \in$ II is biharmonic if and only if it can be written as

$$
f(Z)=h_{0}(Z)+h_{1}(Z) \cdot N(Z)
$$

with $h_{0}$ and $h_{1}$ harmonic. 


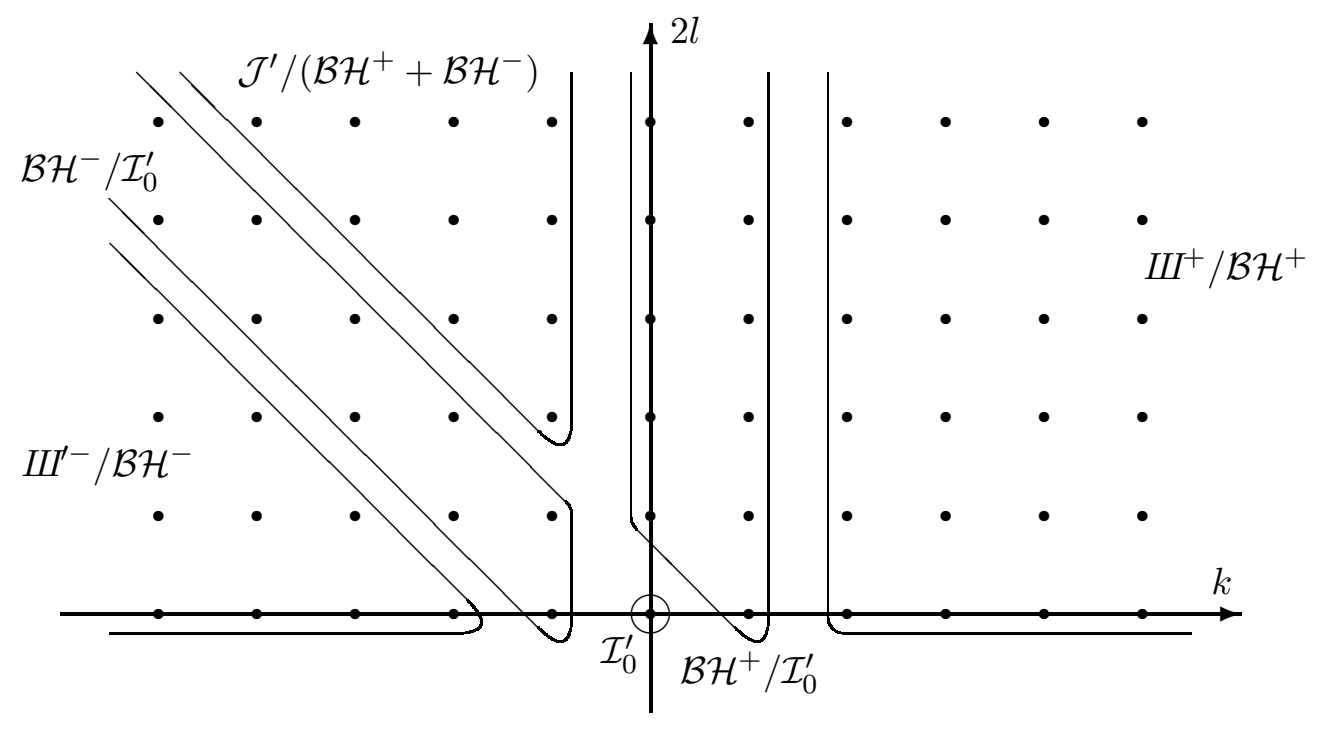

Figure 5: Irreducible components of $\left(\rho^{\prime}, H^{\prime}\right)$.

\section{Realization of Doubly Regular Functions in the Quaternionic Chain Complex}

In this section we decompose ker Mx - which is an invariant subspace of $\left(\rho_{2}^{\prime}, \mathcal{W}^{\prime}\right)$ - into irreducible components. We will see that it has ten irreducible components: five coming from $\left(\rho^{\prime}, W I^{\prime}\right)$ under the map $\partial^{+}$, one trivial one-dimensional representation and four components that are isomorphic to the spaces of doubly regular maps mentioned in Proposition[11, Results of this section will be later used in Subsection 5.2 to decompose $\left(\rho_{2}^{\prime}, \mathcal{W}^{\prime}\right)$ into irreducible components.

\subsection{The Structure of ker $\mathrm{Mx} \subset \mathcal{W}^{\prime}$}

We introduce a $\mathfrak{g l}\left(2, \mathbb{H}_{\mathbb{C}}\right)$-equivariant map on ker $\mathrm{Mx}$; its kernel is automatically an invariant subspace of ker Mx.

Proposition 35. The map $\operatorname{Tr} \circ \partial \circ \square:\left(\rho_{2}^{\prime}, \operatorname{ker} \mathrm{Mx}\right) \rightarrow(\rho, H)$ is $\mathfrak{g l}\left(2, \mathbb{H}_{\mathbb{C}}\right)$-equivariant.

Proof. Recall that the matrices $\left(\begin{array}{ll}1 & 0 \\ 0 & 0\end{array}\right),\left(\begin{array}{ll}0 & B \\ 0 & 0\end{array}\right)$ and $\left(\begin{array}{ll}0 & 0 \\ C & 0\end{array}\right) \in \mathfrak{g l}\left(2, \mathbb{H}_{\mathbb{C}}\right), B, C \in \mathbb{H}_{\mathbb{C}}$, generate $\mathfrak{g l}\left(2, \mathbb{H}_{\mathbb{C}}\right)$. Thus, it is sufficient to show that the map $\operatorname{Tr} \circ \partial \circ \square$ commutes with actions of dilation matrices $\left(\begin{array}{ll}\lambda & 0 \\ 0 & 1\end{array}\right) \in G L\left(2, \mathbb{H}_{\mathbb{C}}\right), \lambda \in \mathbb{R}, \lambda>0$ and $\left(\begin{array}{cc}0 & B \\ 0 & 0\end{array}\right),\left(\begin{array}{ll}0 & 0 \\ C & 0\end{array}\right) \in \mathfrak{g l}\left(2, \mathbb{H}_{\mathbb{C}}\right), B, C \in \mathbb{H}_{\mathbb{C}}$. It is clear from Lemmas 23 and 26 that $\operatorname{Tr} \circ \partial \circ \square$ commutes with the actions of $\left(\begin{array}{ll}0 & B \\ 0 & 0\end{array}\right)$. The dilation matrices $\left(\begin{array}{ll}\lambda & 0 \\ 0 & 1\end{array}\right)$ act by

$$
F(Z) \mapsto \lambda^{-1} \cdot F\left(\lambda^{-1} Z\right) \quad \text { on } \mathcal{W}^{\prime} \quad \text { and } \quad f(Z) \mapsto \lambda^{-4} \cdot f\left(\lambda^{-1} Z\right) \quad \text { on } \amalg \text {, }
$$

and it is clear that $\operatorname{Tr} \circ \partial \circ \square$ commutes with these actions as well.

If $F(Z) \in$ ker Mx, using our previous calculations (32), we obtain:

$$
\operatorname{Tr} \partial\left(\rho_{2}^{\prime}\left(\begin{array}{ll}
0 & 0 \\
C & 0
\end{array}\right) F\right)=\operatorname{Tr}(Z C Z \partial+2 Z C)(\operatorname{Tr} \partial F)-2 \operatorname{Tr}(C F) .
$$


Then we apply $\square=4 \partial \partial^{+}=4\left(\frac{\partial^{2}}{\partial z_{11} \partial z_{22}}-\frac{\partial^{2}}{\partial z_{12} \partial z_{21}}\right)$ :

$$
\begin{gathered}
\operatorname{Tr} \partial \square\left(\rho_{2}^{\prime}\left(\begin{array}{cc}
0 & 0 \\
C & 0
\end{array}\right) F\right)=\operatorname{Tr}(Z C Z \partial+2 Z C)(\operatorname{Tr} \partial \square F)-2 \operatorname{Tr}(C \square F) \\
-4 \operatorname{Tr}\left(C \partial^{+}\right) \operatorname{Tr}(\partial F)+\operatorname{Tr}(C Z+Z C)(\operatorname{Tr} \partial \square F)+8 \operatorname{Tr}\left(C \partial^{+}\right) \operatorname{Tr}(\partial F) \\
=\operatorname{Tr}(Z C Z \partial+4 C Z)(\operatorname{Tr} \partial \square F)+4 \operatorname{Tr}\left(C \partial^{+}\right) \operatorname{Tr}(\partial F)-2 \operatorname{Tr}(C \square F) \\
=\rho\left(\begin{array}{ll}
0 & 0 \\
C & 0
\end{array}\right)(\operatorname{Tr} \partial \square F)+4 \operatorname{Tr}\left(\left(C \partial^{+}+\partial C^{+}\right) \partial F\right)-2 \operatorname{Tr}(C \square F)=\rho\left(\begin{array}{ll}
0 & 0 \\
C & 0
\end{array}\right)(\operatorname{Tr} \partial \square F),
\end{gathered}
$$

since $\mathrm{Mx} F=0$.

We introduce a subspace $\mathcal{M}$ of $\mathcal{W}^{\prime}$ - the kernel of the above equivariant map:

$$
\mathcal{M}=\left\{F \in \mathcal{W}^{\prime} ; \operatorname{Mx} F=0, \operatorname{Tr}(\partial \circ \square F)=0\right\} .
$$

Corollary 36. The subspace $\mathcal{M}$ is invariant under the $\rho_{2}^{\prime}$ action of $\mathfrak{g l}\left(2, \mathbb{H}_{\mathbb{C}}\right)$ on $\mathcal{W}^{\prime}$. All elements of $\mathcal{M}$ are biharmonic (i.e. annihilated by $\square \circ \square$ ).

Proof. The invariance of $\mathcal{M}$ under the $\rho_{2}^{\prime}$ action is immediate from the above proposition. Pick any $F \in \mathcal{M}$. Since $\operatorname{Tr}(\partial \circ \square F)=0$,

$$
0=\square \circ \partial F+\square F^{+} \partial^{+} .
$$

And since $\mathrm{Mx} F=0$,

$$
0=\left(\square \circ \partial F+\square F^{+} \partial^{+}\right) \partial=\frac{1}{2} \square \circ \square F^{+} .
$$

This proves that every element of $\mathcal{M}$ is biharmonic.

Proposition 37. We have: $\partial^{+}\left(H^{\prime}\right)+\mathcal{M}=$ ker Mx.

Proof. Clearly, $\partial^{+}\left(H^{\prime}\right)+\mathcal{M} \subset$ ker Mx. Thus, it is sufficient to prove that the images of $\partial^{+}\left(H^{\prime}\right)$ and ker Mx under the map Tr $\circ \partial \circ \square$ from Proposition 35] are the same. Then, by Corollary 33 we need to show that $\operatorname{Tr}(\partial \circ \square(\operatorname{ker} \mathrm{Mx}))=W^{+} \oplus \mathcal{J} \oplus W^{-}$. And, by Theorem 31 and Proposition 35. it is sufficient to show that $\operatorname{Tr}\left(\partial \circ \square\left(\mathcal{W}^{\prime}\right)\right)$ does not contain $N(Z)^{-1}$ nor $N(Z)^{-3}$. For this purpose we use an identity

$$
\begin{aligned}
& Z \cdot t_{n \underline{m}}^{l}(Z)=\frac{1}{2 l+1}\left(\begin{array}{ll}
(l-n+1) t_{n-\frac{1}{2} \underline{m-\frac{1}{2}}}^{l+\frac{1}{2}}(Z) & (l-n+1) t_{n-\frac{1}{2} \underline{m+\frac{1}{2}}}^{l+\frac{1}{2}}(Z) \\
(l+n+1) t_{n+\frac{1}{2} \underline{m-\frac{1}{2}}}^{l+\frac{1}{2}}(Z) & (l+n+1) t_{n+\frac{1}{2} \underline{m+\frac{1}{2}}}^{l+\frac{1}{2}}(Z)
\end{array}\right)
\end{aligned}
$$

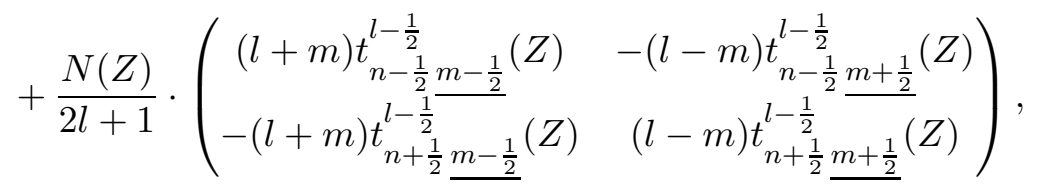

which can be verified using Lemma 23 in [FL1], and equation (33) to check that

$$
\partial_{i j} \circ \square\left(\left(N(Z)^{k} \cdot t_{n \underline{m}}^{l}(Z)\right), \quad i, j=1,2,\right.
$$

is a linear combination of

$$
4 k(2 l+k+1) N(Z)^{k-1} \cdot t_{n \pm \frac{1}{2} \underline{m \pm \frac{1}{2}}}^{l-\frac{1}{2}}(Z), \quad 4 k(2 l+k+1) N(Z)^{k-2} \cdot t_{n \pm \frac{1}{2} \underline{m \pm \frac{1}{2}}}^{l+\frac{1}{2}}(Z) .
$$

But none of these terms can be $N(Z)^{-1}$ or $N(Z)^{-3}$. 
The intersection of $\partial^{+}\left(W^{\prime}\right)$ and $\mathcal{M}$ will be described in Corollary 41. Next, we show that elements of $\mathcal{M}$ have a particular form; this will be used to identify the $K$-types of $\left(\rho_{2}^{\prime}, \mathcal{M}\right)$.

Lemma 38. Let $F_{d}: \mathbb{H}_{\mathbb{C}}^{\times} \rightarrow \mathbb{H}_{\mathbb{C}}$ be homogeneous of degree $d$ and such that $\square F_{d}=0$, then

$$
F(Z)=F_{d}(Z)+\frac{N(Z)}{d+1} \cdot\left(\partial F_{d}(Z) \partial\right)^{+}
$$

satisfies $\operatorname{Mx} F=0$ and $\operatorname{Tr}(\partial \square F)=0$. In particular, if $F_{d}(Z) \in \mathcal{W}^{\prime}$, then $F(Z) \in \mathcal{M}$.

Proof. Note that there are no homogeneous harmonic functions of degree -1 , so division by $d+1$ is permissible. First we check that $\mathrm{Mx} F=0$. Using (15) and the fact that $\partial \partial^{+} F_{d}=0$, we obtain:

$$
\begin{aligned}
& \frac{d+1}{4} \mathrm{Mx} F=(d+1) \partial F_{d} \partial+\partial\left(N(Z) \cdot\left(\partial F_{d} \partial\right)^{+}\right) \partial-\partial \partial^{+}\left(N(Z) \cdot\left(\partial F_{d} \partial\right)\right) \\
& \quad=(d+1) \partial F_{d} \partial+\left(Z^{+} \cdot\left(\partial F_{d} \partial\right)^{+}\right) \partial-\partial\left(Z \cdot\left(\partial F_{d} \partial\right)\right)=(d+1) \partial F_{d} \partial-\partial F_{d} \partial-d \partial F_{d} \partial=0 .
\end{aligned}
$$

Then we check that $\operatorname{Tr}(\partial \square F)=0$ :

$$
\begin{aligned}
& \frac{d+1}{4} \operatorname{Tr}(\partial \square F)=\operatorname{Tr}\left(\partial^{+} \partial \partial\left(N(Z) \cdot\left(\partial F_{d} \partial\right)^{+}\right)\right) \\
& =\operatorname{Tr}\left(\partial^{+} \partial\left(\left(\partial F_{d} \partial\right)^{+} \cdot Z^{+}\right)\right)=-\operatorname{Tr}\left(\partial^{+}\left(\partial F_{d} \partial\right)\right)=0 .
\end{aligned}
$$

Lemma 39. We have1:

$$
\begin{array}{r}
\frac{1}{4} \operatorname{Mx}\left(\begin{array}{cc}
\alpha_{11} t_{n \underline{m}}^{l}(Z) & \alpha_{12} t_{n \underline{m+1}}^{l}(Z) \\
\alpha_{21} t_{n+1 \underline{m}}^{l}(Z) & \alpha_{22} t_{n+1 \underline{m+1}}^{l}(Z)
\end{array}\right)=\partial\left(\begin{array}{cc}
\alpha_{11} t_{n \underline{m}}^{l}(Z) & \alpha_{12} t_{n \underline{m+1}}^{l}(Z) \\
\alpha_{21} t_{n+1 \underline{m}}^{l}(Z) & \alpha_{22} t_{n+1 \underline{m+1}}^{l}(Z)
\end{array}\right) \partial \\
=\left((l-m)\left(\alpha_{11}+\alpha_{21}\right)+(l+m+1)\left(\alpha_{12}+\alpha_{22}\right)\right) \\
\times\left(\begin{array}{cc}
(l-m-1) t_{n+1}^{l-1} \underline{m+1}(Z) & (l-m-1) t_{n}^{l-1}(Z) \\
(l+m) t_{n+1}^{l-1}(Z) & (l+m) t_{n \underline{m}}^{l-1}(Z)
\end{array}\right)
\end{array}
$$

and, if $l>1$,

$$
\begin{aligned}
& \frac{1}{4} \operatorname{Mx}\left(N(Z) \cdot\left(\begin{array}{cc}
\beta_{11} t_{n \underline{m}}^{l-1}(Z) & \beta_{12} t_{n-\underline{m+1}}^{l-1}(Z) \\
\beta_{21} t_{n+1}^{l-1}(Z) & \beta_{22} t_{n+1}^{l-1} \underline{\underline{m+1}}(Z)
\end{array}\right)\right) \\
& =3 l\left(\begin{array}{cc}
-\beta_{22} t_{n+1}^{l-1}(Z) & \beta_{12} t_{n+\frac{m+1}{l-1}}^{l-1}(Z) \\
\beta_{21} t_{n+1 \underline{m}}^{l-1}(Z) & -\beta_{11} \bar{t}_{n \underline{m}}^{l-1}(Z)
\end{array}\right)+\frac{1}{l-1}\left(\begin{array}{cc}
\gamma_{11} t_{n+1 \underline{m+1}}^{l-1}(Z) & \gamma_{12} t_{n \underline{m+1}}^{l-1}(Z) \\
\gamma_{21} t_{n+1 \underline{m}}^{l-1}(Z) & \gamma_{22} t_{n \underline{m}}^{l-1}(Z)
\end{array}\right) \\
& +\frac{N(Z) \cdot l}{l-1}\left((l-m-1)\left(\beta_{11}+\beta_{21}\right)+(l+m)\left(\beta_{12}+\beta_{22}\right)\right) \\
& \times\left(\begin{array}{cc}
(l-m-2) t_{n+1}^{l-2} \underline{m+1}(Z) & (l-m-2) t_{n \underline{m+1}}^{l-2}(Z) \\
(l+m-1) t_{n+1 \underline{m}}^{l-2}(Z) & (l+m-1) t_{n \underline{m}}^{l-2}(Z)
\end{array}\right),
\end{aligned}
$$

\footnotetext{
${ }^{1}$ In this formula, whenever the indices of, say, $\alpha_{11} t_{n \underline{\underline{m}}}^{l}(Z)$ happen to be outside of the allowed range $l=$ $0, \frac{1}{2}, 1, \frac{3}{2}, \ldots,-l \leq m, n \leq l$, the coefficient $\alpha_{11}$ must be set to be zero. The same considerations apply to other formulas in this Lemma.
} 
where the coefficients

$$
\begin{aligned}
& \gamma_{11}=(l-m-1)(l+n) \beta_{11}+(m+1)(l+n) \beta_{12}+(l-m-1)(n+1) \beta_{21}+(m+1)(n+1) \beta_{22}, \\
& \gamma_{12}=(l-m-1) n \beta_{11}+(m+1) n \beta_{12}-(l-m-1)(l-n-1) \beta_{21}-(m+1)(l-n-1) \beta_{22}, \\
& \gamma_{21}=m(l+n) \beta_{11}-(l+m)(l+n) \beta_{12}+m(n+1) \beta_{21}-(l+m)(n+1) \beta_{22}, \\
& \gamma_{22}=m n \beta_{11}-(l+m) n \beta_{12}-m(l-n-1) \beta_{21}+(l+m)(l-n-1) \beta_{22} ;
\end{aligned}
$$

in the special case of $l=1$,

$$
\frac{1}{4} \operatorname{Mx}\left(N(Z) \cdot\left(\begin{array}{cc}
\beta_{11} t_{n \underline{m}}^{0}(Z) & \beta_{12} t_{n}^{0} \underline{m+1}(Z) \\
\beta_{21} t_{n+1 \underline{m}}^{0}(Z) & \beta_{22} t_{n+1 \underline{m+1}}^{0}(Z)
\end{array}\right)\right)=3\left(\begin{array}{cc}
-\beta_{22} t_{n+1}^{0}{ }_{n+1}^{m}(Z) & \beta_{12} t_{n}^{0} \underline{m+1}(Z) \\
\beta_{21} t_{n+1 \underline{m}}^{0}(Z) & -\beta_{11} t_{n \underline{m}}^{0}(Z)
\end{array}\right) .
$$

Similarly,

$$
\begin{aligned}
& \frac{1}{4} \operatorname{Mx}\left(\frac{1}{N(Z)}\left(\begin{array}{cc}
\alpha_{11}^{\prime} t_{n+1}^{l} \underline{m+1}\left(Z^{-1}\right) & \alpha_{12}^{\prime} t_{n m+1}^{l}\left(Z^{-1}\right) \\
\alpha_{21}^{\prime} t_{n+1 \underline{m}}^{l}\left(Z^{-1}\right) & \alpha_{22}^{\prime} t_{n \underline{m}}^{l}\left(Z^{-1}\right)
\end{array}\right)\right) \\
& =\left((l-n)\left(\alpha_{11}^{\prime}+\alpha_{21}^{\prime}\right)+(l+n+1)\left(\alpha_{12}^{\prime}+\alpha_{22}^{\prime}\right)\right) \\
& \times \frac{1}{N(Z)}\left(\begin{array}{cc}
(l-n+1) t_{n}^{l+1}\left(Z^{-1}\right) & (l-n+1) t_{n \underline{m+1}}^{l+1}\left(Z^{-1}\right) \\
(l+n+2) t_{n+1}^{l+\underline{m}}\left(Z^{-1}\right) & (l+n+2) t_{n+1}^{l+1} \underline{m+1}\left(Z^{-1}\right)
\end{array}\right)
\end{aligned}
$$

and, if $l \geq-1 / 2$,

$$
\begin{aligned}
& \frac{1}{4} \operatorname{Mx}\left(\begin{array}{cc}
\beta_{11}^{\prime} t_{n+1}^{l+1}\left(Z^{-1}\right) & \beta_{12}^{\prime} t_{n \underline{m+1}}^{l+1}\left(Z^{-1}\right) \\
\beta_{21}^{\prime} t_{n+1 \underline{m}}^{l+1}\left(Z^{-1}\right) & \beta_{22}^{\prime} t_{n \underline{m}}^{l+1}\left(Z^{-1}\right)
\end{array}\right) \\
& =\frac{3(l+1)}{N(Z)}\left(\begin{array}{cc}
\beta_{22}^{\prime} t_{n \underline{m}}^{l+1}\left(Z^{-1}\right) & -\beta_{12}^{\prime} t_{n \underline{m+1}}^{l+1}\left(Z^{-1}\right) \\
-\beta_{21}^{\prime} t_{n+1}^{l+1}\left(Z^{-1}\right) & \beta_{11}^{\prime} t_{n+1}^{l+1} \underline{\underline{m+1}}\left(Z^{-1}\right)
\end{array}\right) \\
& +\frac{N(Z)^{-1}}{l+2}\left(\begin{array}{cc}
\gamma_{11}^{\prime} t_{n \underline{\underline{m}}}^{l+1}\left(Z^{-1}\right) & \gamma_{12}^{\prime} t_{n \underline{m+1}}^{l+1}\left(Z^{-1}\right) \\
\gamma_{21}^{\prime} t_{n+1}^{l+1} \underline{\underline{m}}\left(Z^{-1}\right) & \gamma_{22}^{\prime} t_{n+1}^{l+1} \underline{\underline{m+1}}\left(Z^{-1}\right)
\end{array}\right) \\
& +\frac{l+1}{l+2}\left((l-n+1)\left(\beta_{11}^{\prime}+\beta_{21}^{\prime}\right)+(l+n+2)\left(\beta_{12}^{\prime}+\beta_{22}^{\prime}\right)\right) \\
& \times\left(\begin{array}{cc}
(l-n+2) t_{n \underline{\underline{m}}}^{l+2}\left(Z^{-1}\right) & (l-n+2) t_{n \underline{m+1}}^{l+2}\left(Z^{-1}\right) \\
(l+n+3) t_{n+1 \underline{\underline{m}}}^{l+2}\left(Z^{-1}\right) & (l+n+3) t_{n+1 \underline{m+1}}^{l+2}\left(Z^{-1}\right)
\end{array}\right),
\end{aligned}
$$

where the coefficients

$$
\begin{aligned}
\gamma_{11}^{\prime}= & -(l+m+2)(l-n+1) \beta_{11}^{\prime}-(l+m+2) n \beta_{12}^{\prime}-m(l-n+1) \beta_{21}^{\prime}-m n \beta_{22}^{\prime}, \\
\gamma_{12}^{\prime}=- & (m+1)(l-n+1) \beta_{11}^{\prime}-(m+1) n \beta_{12}^{\prime}+(l-m+1)(l-n+1) \beta_{21}^{\prime}+(l-m+1) n \beta_{22}^{\prime}, \\
\gamma_{21}^{\prime}=- & (l+m+2)(n+1) \beta_{11}^{\prime}+(l+m+2)(l+n+2) \beta_{12}^{\prime}-m(n+1) \beta_{21}^{\prime}+m(l+n+2) \beta_{22}^{\prime}, \\
\gamma_{22}^{\prime}=- & (m+1)(n+1) \beta_{11}^{\prime}+(m+1)(l+n+2) \beta_{12}^{\prime}+(l-m+1)(n+1) \beta_{21}^{\prime} \\
& \quad-(l-m+1)(l+n+2) \beta_{22}^{\prime} .
\end{aligned}
$$

Proof. The result is obtained by rather tedious, yet completely straightforward calculations using equation (34), Lemma 22 from [FL1] and identity

$$
\begin{aligned}
& \partial\left(N(Z)^{-1} \cdot t_{n \underline{m}}^{l}\left(Z^{-1}\right)\right) \\
& =-\frac{1}{N(Z)}\left(\begin{array}{ll}
(l-n+1) t_{n-\frac{1}{2} \underline{m-\frac{1}{2}}}^{l+\frac{1}{2}}\left(Z^{-1}\right) & (l-n+1) t_{n-\frac{1}{2} \underline{m+\frac{1}{2}}}^{l+\frac{1}{2}}\left(Z^{-1}\right) \\
(l+n+1) t_{n+\frac{1}{2} \underline{m-\frac{1}{2}}}^{l+\frac{1}{2}}\left(Z^{-1}\right) & (l+n+1) t_{n+\frac{1}{2} \underline{m+\frac{1}{2}}}^{l+\frac{1}{2}}\left(Z^{-1}\right)
\end{array}\right),
\end{aligned}
$$

which in turn can be verified using Lemmas 22 and 23 in [FL1. 
Proposition 40. We have:

$$
\mathcal{M} \oplus \mathbb{C} \text {-span of }\{N(Z) \cdot Z\}=\left\{F \in \mathcal{W}^{\prime} ; \operatorname{Mx} F=0,(\square \circ \square) F=0\right\} .
$$

Moreover, every element $A \in \mathcal{M}$ is a linear combination of $N(Z)^{-1} \cdot Z$ and homogeneous elements of the form

$$
A_{d}(Z)+\frac{N(Z)}{d+1} \cdot\left(\partial A_{d}(Z) \partial\right)^{+} \in \mathcal{M}
$$

for some $A_{d}: \mathbb{H}_{\mathbb{C}}^{\times} \rightarrow \mathbb{H}_{\mathbb{C}}$ which is homogeneous of degree $d$ and harmonic.

In particular, the space $\mathcal{M}$ can be characterized as the unique maximal $\mathfrak{g l}\left(2, \mathbb{H}_{\mathbb{C}}\right)$-invariant subspace of ker Mx consisting of biharmonic functions.

Proof. It is easy to see that $N(Z)^{-1} \cdot Z \in \mathcal{M}$ and $N(Z) \cdot Z \notin \mathcal{M}$. Let

$$
\mathcal{M}^{\prime}=\left\{F \in \mathcal{W}^{\prime} ; \operatorname{Mx} F=0,(\square \circ \square) F=0\right\} .
$$

By Corollary [36,

$$
\mathcal{M} \oplus \mathbb{C} \text {-span of }\{N(Z) \cdot Z\} \subset \mathcal{M}^{\prime},
$$

and we need to prove the opposite inclusion. By Lemma 38 it is sufficient to show that every element $A \in \mathcal{M}^{\prime}$ is a linear combination of $N(Z) \cdot Z, N(Z)^{-1} \cdot Z$ and homogeneous elements of the form (38). By Proposition 34, $A(Z)$ has to be a linear combination of homogeneous elements that appear in Lemma 39

$$
\begin{aligned}
& \left(\begin{array}{cc}
\alpha_{11} t_{n \underline{m}}^{l}(Z) & \alpha_{12} t_{n \underline{m+1}}^{l}(Z) \\
\alpha_{21} t_{n+1 \underline{m}}^{l}(Z) & \alpha_{22} t_{n+1}^{l} \underline{\underline{m+1}}(Z)
\end{array}\right), \quad N(Z) \cdot\left(\begin{array}{cc}
\beta_{11} t_{n \underline{m}}^{l-1}(Z) & \beta_{12} t_{n \underline{m+1}}^{l-1}(Z) \\
\beta_{21} t_{n+1 \underline{m}}^{l-1}(Z) & \beta_{22} t_{n+1 \underline{m+1}}^{l-1}(Z)
\end{array}\right),
\end{aligned}
$$

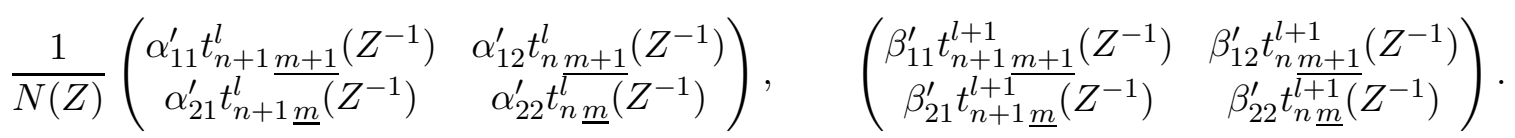

Our element $A(Z) \in \mathcal{M}^{\prime}$ must also be annihilated by $\mathrm{Mx}$, and, in view of Lemma 39, without loss of generality we may assume that it is either a linear combination of the first two or the last two types. We provide a sketch for the first case with $l \neq 1$ only, the subcase $l=1$ and other case are similar. Thus we assume

$$
A(Z)=\left(\begin{array}{cc}
\alpha_{11} t_{n \underline{m}}^{l}(Z) & \alpha_{12} t_{n \underline{m+1}}^{l}(Z) \\
\alpha_{21} t_{n+1 \underline{m}}^{l}(Z) & \alpha_{22} t_{n+1 \underline{m+1}}^{l}(Z)
\end{array}\right)+N(Z) \cdot\left(\begin{array}{cc}
\beta_{11} t_{n \underline{m}}^{l-1}(Z) & \beta_{12} t_{n \underline{m+1}}^{l-1}(Z) \\
\beta_{21} t_{n+1 \underline{m}}^{l-1}(Z) & \beta_{22} t_{n+1 \underline{m+1}}^{l-1}(Z)
\end{array}\right) .
$$

If the second summand is zero, then $A(Z)$ is harmonic with $\partial A(Z) \partial=0$ (since $\mathrm{Mx} A=0$ ), and $A(Z)$ is of the form (38). Thus we further assume

$$
N(Z) \cdot\left(\begin{array}{cc}
\beta_{11} t_{n \underline{\underline{m}}}^{l-1}(Z) & \beta_{12} t_{n \underline{m+1}}^{l-1}(Z) \\
\beta_{21} t_{n+1 \underline{m}}^{l-1}(Z) & \beta_{22} t_{n+1 \underline{m+1}}^{l-1}(Z)
\end{array}\right) \neq 0
$$

and that $-l+1 \leq m, n \leq l-2$ so that $t_{n \underline{m}}^{l-1}(Z), t_{n \underline{m+1}}^{l-1}(Z), t_{n+1}^{l-1}(Z), t_{n+1 \underline{m+1}}^{l-1}(Z) \neq 0$. (The case when some of these functions are zero needs to be considered separately.) Since $\mathrm{Mx} A=0$, Mx $A$ has no harmonic component. This means that in the harmonic component of (36) the coefficients in the same rows are equal and proportional to the coefficients in (35):

$$
\left\{\begin{array}{l}
-3 l(l-1) \beta_{22}+\gamma_{11}=3 l(l-1) \beta_{12}+\gamma_{12} \\
3 l(l-1) \beta_{21}+\gamma_{21}=-3 l(l-1) \beta_{11}+\gamma_{22} \\
(l+m)\left(-3 l(l-1) \beta_{22}+\gamma_{11}\right)=(l-m-1)\left(3 l(l-1) \beta_{21}+\gamma_{21}\right) .
\end{array}\right.
$$


The first two equations simplify to

$$
\left\{\begin{array}{l}
l\left((l-m-1)\left(\beta_{11}+\beta_{21}\right)-(3 l-m-4)\left(\beta_{12}+\beta_{22}\right)\right)=0 \\
l\left((3 l+m-3)\left(\beta_{11}+\beta_{21}\right)-(l+m)\left(\beta_{12}+\beta_{22}\right)\right)=0,
\end{array}\right.
$$

and

$$
\operatorname{det}\left(\begin{array}{cc}
l-m-1 & -3 l+m+4 \\
3 l+m-3 & -(l+m)
\end{array}\right)=4(l-1)(2 l-3)
$$

Assume for now that $l \neq 3 / 2$, then $\beta_{11}+\beta_{21}=0$ and $\beta_{12}+\beta_{22}=0$. Substituting $\beta_{21}=-\beta_{11}$ and $\beta_{12}=-\beta_{22}$ into the third equation yields

$$
4 l(l-1)\left((l-m-1) \beta_{11}-(l+m) \beta_{22}\right)=0,
$$

hence $\left(\beta_{11}, \beta_{22}\right)$ is proportional to $(l+m, l-m-1)$. It follows from $\mathrm{Mx} A(Z)=0$ that $A(Z)$ is of the form (38).

In the exceptional case $l=3 / 2$, the system (40) has rank one and simplifies to a single equation $\beta_{11}+\beta_{21}=\beta_{12}+\beta_{22}$. If $m=n=-1 / 2$ we get exactly one additional linearly independent solution in $\mathcal{M}^{\prime}$ that is not in $\mathcal{M}$ :

$$
\beta_{11}=\beta_{12}=\beta_{21}=\beta_{22}=1, \quad A(Z)=N(Z) \cdot Z .
$$

Finally, to prove the maximality property of $\mathcal{M}$, it is sufficient to show that a $\mathfrak{g l}\left(2, \mathbb{H}_{\mathbb{C}}\right)$ invariant subspace of $\mathcal{M}^{\prime}$ cannot contain an element of the form $A+N(Z) \cdot Z$ with $A \in \mathcal{M}$. Indeed, $N(Z) \cdot Z$ is the image of an element $\frac{1}{2} N(Z)^{2}$ under a map $\partial^{+}: W^{\prime} \rightarrow \mathcal{W}^{\prime}$, which is $\mathfrak{g l}\left(2, \mathbb{H}_{\mathbb{C}}\right)$-equivariant by Proposition 27 . Since $\frac{1}{2} N(Z)^{2}$ generates $H^{+}$(Theorem $\underline{32}$ ), $N(Z) \cdot Z$ generates $\partial^{+}\left(\amalg^{+}\right)$, which contains $N(Z)^{2} \cdot Z \notin \mathcal{M}^{\prime}$. Therefore, a $\mathfrak{g l}\left(2, \mathbb{H}_{\mathbb{C}}\right)$-invariant subspace of $\mathcal{W}^{\prime}$ containing $A+N(Z) \cdot Z$ with $A \in \mathcal{M}$ also contains elements not in $\mathcal{M}^{\prime}$.

Corollary 41. The intersection of the image of the map $\partial^{+}: H^{\prime} \rightarrow \mathcal{W}^{\prime}$ and $\mathcal{M}$ in $\mathcal{W}^{\prime}$ is precisely $\partial^{+}\left(\mathcal{B H}^{+}\right) \oplus \partial^{+}\left(\mathcal{B H}^{-}\right)$.

Proof. The result follows from Proposition 27, Theorem 32 and Proposition 40.

\subsection{The $K$-types of $\mathcal{M}$}

In this subsection we describe the $K$-types of $\mathcal{M}$. For $d \in \mathbb{Z}$, define

$$
\mathcal{M}(d)=\{A(Z) \in \mathcal{M} ; A(Z) \text { is homogeneous of degree } d\} .
$$

We realize $\mathfrak{s l}(2, \mathbb{C}) \times \mathfrak{s l}(2, \mathbb{C})$ as diagonal elements of $\mathfrak{g l}\left(2, \mathbb{H}_{\mathbb{C}}\right)$ :

$$
\mathfrak{s l}(2, \mathbb{C}) \times \mathfrak{s l}(2, \mathbb{C})=\left\{\left(\begin{array}{cc}
A & 0 \\
0 & D
\end{array}\right) \in \mathfrak{g l}\left(2, \mathbb{H}_{\mathbb{C}}\right) ; A, D \in \mathbb{H}_{\mathbb{C}}, \operatorname{Re}(A)=\operatorname{Re}(D)=0\right\} .
$$

Proposition 42. Each $\mathcal{M}(d)$ is invariant under the $\rho_{2}^{\prime}$ action restricted to $\mathfrak{s l}(2, \mathbb{C}) \times \mathfrak{s l}(2, \mathbb{C})$, and we have the following decomposition into irreducible components:

$$
\begin{gathered}
\mathcal{M}(-1)=V_{0} \otimes V_{0}=\mathbb{C} \text {-span of }\left\{N(Z)^{-1} \cdot Z\right\}, \\
\mathcal{M}(2 l)=\left(V_{l-\frac{1}{2}} \otimes V_{l-\frac{1}{2}}\right) \oplus\left(V_{l+\frac{1}{2}} \otimes V_{l-\frac{1}{2}}\right) \oplus\left(V_{l-\frac{1}{2}} \otimes V_{l+\frac{1}{2}}\right) \oplus\left(V_{l+\frac{1}{2}} \otimes V_{l+\frac{1}{2}}\right), \\
\mathcal{M}(-2 l-2)=\left(V_{l-\frac{1}{2}} \otimes V_{l-\frac{1}{2}}\right) \oplus\left(V_{l+\frac{1}{2}} \otimes V_{l-\frac{1}{2}}\right) \oplus\left(V_{l-\frac{1}{2}} \otimes V_{l+\frac{1}{2}}\right) \oplus\left(V_{l+\frac{1}{2}} \otimes V_{l+\frac{1}{2}}\right),
\end{gathered}
$$


$l=0, \frac{1}{2}, 1, \frac{3}{2}, \ldots$ Explicitly, these irreducible components are generated by homogeneous elements of the form (38) with harmonic parts:

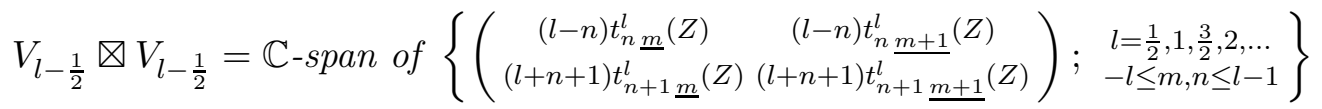

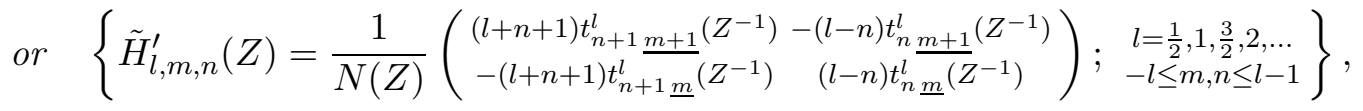

$$
\begin{aligned}
& V_{l+\frac{1}{2}} \otimes V_{l-\frac{1}{2}}=\mathbb{C} \text {-span of }\left\{\tilde{G}_{l, m, n}(Z)=\left(\begin{array}{cc}
t_{n}^{l} \underline{m}(Z) & t_{n}^{l} \underline{m+1}_{m}(Z) \\
-t_{n+1}^{l} \underline{m} & -t_{n+1}^{\underline{m+1}}(Z)
\end{array}\right) ; \begin{array}{c}
l=\frac{1}{2}, 1, \frac{3}{2}, 2, \ldots \\
-l \leq m \leq l-1 \\
-l-1 \leq n \leq l
\end{array}\right\} \\
& \text { or } \quad\left\{\tilde{F}_{l, m, n}^{\prime}(Z)=\frac{1}{N(Z)}\left(\begin{array}{c}
(l-m)(l+n+1) t_{n+1}^{l} \frac{m+1}{(}\left(Z^{-1}\right)-(l-m)(l-n) t_{n}^{l} \underline{m+1}\left(Z^{-1}\right) \\
(l+m+1)(l+n+1) t_{n+1}^{l} \underline{\underline{m}}\left(Z^{-1}\right)-(l+m+1)(l-n) t_{n \underline{m}}^{l}\left(Z^{-1}\right)
\end{array}\right) ; \begin{array}{c}
l=\frac{1}{2}, 1, \frac{3}{2}, 2, \ldots \\
-l-1 \leq m \leq l \\
-l \leq n \leq l-1
\end{array}\right\}, \\
& V_{l-\frac{1}{2}} \otimes V_{l+\frac{1}{2}}=\mathbb{C} \text {-span of }
\end{aligned}
$$

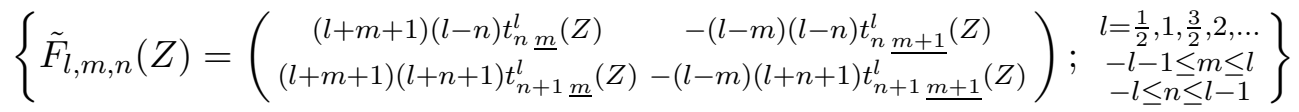

$$
\begin{aligned}
& \text { or } \quad\left\{\tilde{G}_{l, m, n}^{\prime}(Z)=\frac{1}{N(Z)}\left(\begin{array}{cc}
t_{n+1}^{l} \underline{m+1} & \left(Z^{-1}\right) t_{n}^{l}{ }_{m+1}^{m}\left(Z^{-1}\right) \\
-t_{n+1}^{l} \underline{m} & \left(Z^{-1}\right)-\bar{t}_{n \underline{m}}^{l}\left(Z^{-1}\right)
\end{array}\right) ; \begin{array}{c}
l=\frac{1}{2}, 1, \frac{3}{2}, 2, \ldots \\
-l \leq m \leq l-1 \\
-l-1 \leq n \leq l
\end{array}\right\},
\end{aligned}
$$

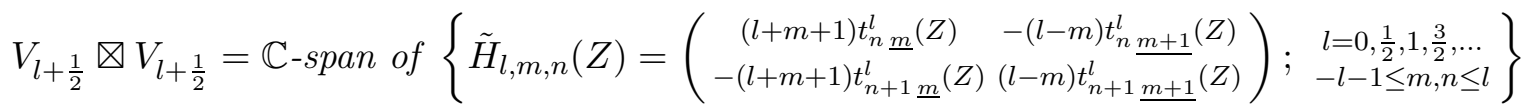

$$
\begin{aligned}
& \text { or } \quad\left\{\frac{1}{N(Z)}\left(\begin{array}{cc}
(l-m) t_{n+1}^{l} \frac{m+1}{m}\left(Z^{-1}\right) & (l-m) t_{n}^{l} \frac{m+1}{(l+m+1) t_{n+1}^{l} \underline{\underline{m}}}\left(Z^{-1}\right)(l+m+1) t_{n \underline{m}}^{l}\left(Z^{-1}\right)
\end{array}\right) ; \begin{array}{c}
l=0, \frac{1}{2}, 1, \frac{3}{2}, \ldots \\
-l-1 \leq m, n \leq l
\end{array}\right\} \text {. }
\end{aligned}
$$

The functions in $\mathcal{M}(2 l)$ that lie in

$$
\left(V_{l+\frac{1}{2}} \otimes V_{l-\frac{1}{2}}\right) \oplus\left(V_{l-\frac{1}{2}} \otimes V_{l+\frac{1}{2}}\right) \oplus\left(V_{l+\frac{1}{2}} \otimes V_{l+\frac{1}{2}}\right)
$$

and the functions in $\mathcal{M}(-2 l-2)$ that lie in

$$
\left(V_{l-\frac{1}{2}} \otimes V_{l-\frac{1}{2}}\right) \oplus\left(V_{l+\frac{1}{2}} \otimes V_{l-\frac{1}{2}}\right) \oplus\left(V_{l-\frac{1}{2}} \otimes V_{l+\frac{1}{2}}\right)
$$

have harmonic parts only, their non-harmonic parts are zero.

Proof. Note that, as usual, if the indices of $t_{n \underline{\underline{m}}}^{l}(Z)$ happen to be outside of the allowed range $l=0, \frac{1}{2}, 1, \frac{3}{2}, \ldots, m, n \in \mathbb{Z}+l,-l \leq m, n \leq l$, then such matrix coefficients are declared to be zero. The result follows from Proposition 40, Lemma 26 and explicit realization of the isomorphism of representations of $\mathfrak{s l}(2, \mathbb{C})$

$$
V_{l} \otimes V_{\frac{1}{2}} \simeq V_{l-\frac{1}{2}} \oplus V_{l+\frac{1}{2}}
$$

The assertion about non-harmonic parts follows from Lemma 39 ,

Combining this result with Corollary 41 and comparing the decompositions into the $\mathfrak{s l}(2, \mathbb{C}) \times$ $\mathfrak{s l}(2, \mathbb{C})$ components, we obtain: 


\section{Corollary 43.}

$$
\begin{aligned}
& \partial^{+}\left(\mathcal{B H}^{+}\right)=\bigoplus_{l \geq 0}\left(V_{l-\frac{1}{2}} \otimes V_{l-\frac{1}{2}} \text { component of } \mathcal{M}(2 l)\right) \oplus\left(V_{l+\frac{1}{2}} \otimes V_{l+\frac{1}{2}} \text { component of } \mathcal{M}(2 l)\right), \\
& \begin{aligned}
\partial^{+}\left(\mathcal{B H}^{-}\right) \\
\quad=\bigoplus_{l \geq 0}\left(V_{l-\frac{1}{2}} \otimes V_{l-\frac{1}{2}} \text { comp. of } \mathcal{M}(-2 l-2)\right) \oplus\left(V_{l+\frac{1}{2}} \otimes V_{l+\frac{1}{2}} \text { comp. of } \mathcal{M}(-2 l-2)\right) .
\end{aligned}
\end{aligned}
$$

Lemma 44. The functions $\left(\tilde{F}_{l, m, n}\right)^{+},\left(\tilde{F}_{l, m, n}^{\prime}\right)^{+}: \mathbb{H}_{\mathbb{C}} \rightarrow \mathbb{H}_{\mathbb{C}}$ are left regular. The functions $\left(\tilde{G}_{l, m, n}\right)^{+},\left(\tilde{G}_{l, m, n}^{\prime}\right)^{+}: \mathbb{H}_{\mathbb{C}} \rightarrow \mathbb{H}_{\mathbb{C}}$ are right regular. The functions $\left(\tilde{H}_{l, m, n}\right)^{+},\left(\tilde{H}_{l, m, n}^{\prime}\right)^{+}: \mathbb{H}_{\mathbb{C}} \rightarrow$ $\mathbb{H}_{\mathbb{C}}$ are both left and right regular.

Proof. The result follows by comparing the columns and rows of the functions in question with the basis of left and right regular functions given in Proposition 24 in [FL1].

The harmonic functions

$$
\tilde{F}_{l, m, n}(Z), \tilde{F}_{l, m, n}^{\prime}(Z), \tilde{G}_{l, m, n}(Z), \tilde{G}_{l, m, n}^{\prime}(Z), \tilde{H}_{l, m, n}(Z), \tilde{H}_{l, m, n}^{\prime}(Z)
$$

belong to $\mathcal{M}$. We complete these and $N(Z)^{-1} \cdot Z$ to a basis of $\mathcal{M}$ by letting $\tilde{I}_{l, m, n}(Z)$ be the elements in $\mathcal{M}$ that have harmonic parts

$$
\left(\begin{array}{cc}
(l-n) t_{n \underline{m}}^{l}(Z) & (l-n) t_{n n+\frac{m+1}{l}}^{l}(Z) \\
(l+n+1) t_{n+1 \underline{m}}^{l}(Z) & (l+n+1) t_{n+1}^{l} \underline{m+1}(Z)
\end{array}\right), \quad \begin{aligned}
& l=\frac{1}{2}, 1, \frac{3}{2}, 2, \ldots, \\
& -l \leq m, n \leq l-1
\end{aligned},
$$

these generate the $V_{l-\frac{1}{2}} \otimes V_{l-\frac{1}{2}}$ component of $\mathcal{M}(2 l)$, and $\tilde{I}_{l, m, n}^{\prime}(Z)$ be the elements in $\mathcal{M}$ that have harmonic parts

$$
\frac{1}{N(Z)}\left(\begin{array}{cc}
(l-m) t_{n+1}^{l}{ }_{n+1}^{m}\left(Z^{-1}\right) & (l-m) t_{n \underline{m+1}}^{l}\left(Z^{-1}\right) \\
(l+m+1) t_{n+1 \underline{m}}^{l}\left(Z^{-1}\right) & (l+m+1) t_{n \underline{m}}^{l}\left(Z^{-1}\right)
\end{array}\right), \quad \begin{gathered}
l=0, \frac{1}{2}, 1, \frac{3}{2}, \ldots \\
-l-1 \leq m, n \leq l
\end{gathered}
$$

these generate the $V_{l+\frac{1}{2}} \otimes V_{l+\frac{1}{2}}$ component of $\mathcal{M}(-2 l-2)$. The following technical lemma will be used to construct equivariant maps $\tau_{a}^{ \pm}$and $\tau_{s}^{ \pm}$from $\mathcal{W}^{\prime}$ to doubly regular functions.

Lemma 45. We have the following identities:

$$
\begin{array}{r}
\left(\tilde{F}_{l, m, n} \overleftarrow{\partial}\right)(Z)=\left(\tilde{F}_{l, m, n}^{\prime} \overleftarrow{\partial}\right)(Z)=\vec{\partial} \tilde{G}_{l, m, n}(Z)=\vec{\partial} \tilde{G}_{l, m, n}^{\prime}(Z)=0 \\
\vec{\partial} \tilde{H}_{l, m, n}(Z)=\left(\tilde{H}_{l, m, n} \overleftarrow{\partial}\right)(Z)=\vec{\partial} \tilde{H}_{l, m, n}^{\prime}(Z)=\left(\tilde{H}_{l, m, n}^{\prime} \overleftarrow{\partial}\right)(Z)=0 \\
\vec{\partial}\left(N(Z)^{-1} \cdot Z\right)=\left(N(Z)^{-1} \cdot Z\right) \overleftarrow{\partial}=N(Z)^{-1} \\
\vec{\partial} \tilde{F}_{l, m, n}(Z)=(2 l+1)\left(\begin{array}{c}
(l-m)(l+m+1) t_{n+\frac{1}{2}}^{l-\frac{1}{2}} \frac{m+\frac{1}{2}}{2}(Z)-(l-m-1)(l-m) t_{n+\frac{1}{2}}^{l-\frac{1}{2}} \frac{m+\frac{3}{2}}{2}(Z) \\
(l+m)(l+m+1) t_{n+\frac{1}{2}}^{l-\frac{1}{2}}(Z)-(l+m+1)(l-m) t_{n+\frac{1}{2}}^{l-\frac{1}{2}} \frac{m+\frac{1}{2}}{(2 l}(Z)
\end{array}\right) \\
=(2 l+1) Z^{-1} \cdot \tilde{F}_{l, m, n}(Z)
\end{array}
$$




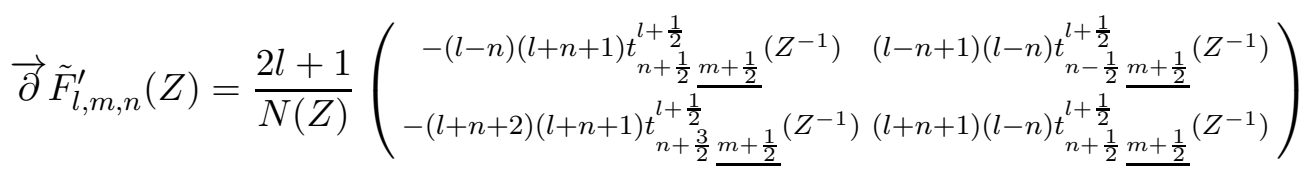

$$
\begin{aligned}
& =-(2 l+1) Z^{-1} \cdot \tilde{F}_{l, m, n}^{\prime}(Z), \\
& \left(\tilde{G}_{l, m, n} \overleftarrow{\partial}\right)(Z)=(2 l+1)\left(\begin{array}{cc}
t_{n+\frac{1}{2}}^{l-\frac{1}{2}} \frac{m+\frac{1}{2}}{(Z)} & t_{n-\frac{1}{2}}^{l-\frac{1}{2}} \underline{m+\frac{1}{2}}(Z) \\
-t_{n+\frac{3}{2}}^{l-\frac{1}{2}} \underline{m+\frac{1}{2}}(Z) & -t_{n+\frac{1}{2}}^{l-\frac{1}{2}} \underline{\underline{m+\frac{1}{2}}}(Z)
\end{array}\right)=(2 l+1) G_{l, m, n}(Z) \cdot Z^{-1}
\end{aligned}
$$

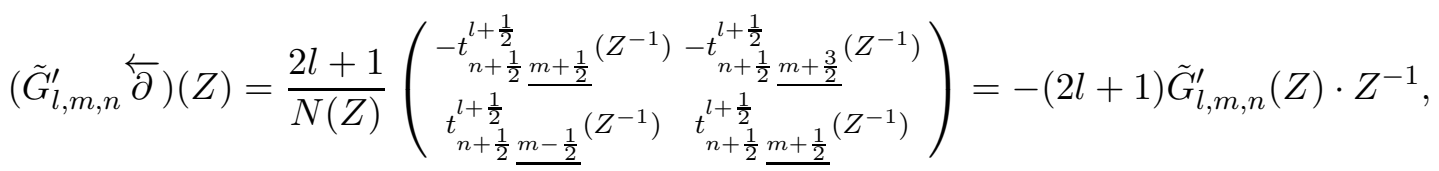

$$
\begin{aligned}
& \vec{\partial} \tilde{I}_{l, m, n}(Z)=(2 l+1) Z^{-1} \cdot \tilde{I}_{l, m, n}(Z), \quad\left(\tilde{I}_{l, m, n} \overleftarrow{\partial}\right)(Z)=(2 l+1) \tilde{I}_{l, m, n}(Z) \cdot Z^{-1} \\
& \vec{\partial} \tilde{I}_{l, m, n}^{\prime}(Z)=-(2 l+1) Z^{-1} \cdot \tilde{I}_{l, m, n}^{\prime}(Z), \quad\left(\tilde{I}_{l, m, n}^{\prime} \overleftarrow{\partial}\right)(Z)=-(2 l+1) \tilde{I}_{l, m, n}^{\prime}(Z) \cdot Z^{-1}
\end{aligned}
$$

Proof. The result follows by direct computations from Lemmas 22, 23 from [FL1] and identity (37).

\subsection{Equivariant Maps from $\mathcal{W}^{\prime}$ to Doubly Regular Functions}

The goal of this subsection is to construct equivariant maps $\tau_{a}^{ \pm}$and $\tau_{s}^{ \pm}$from $\mathcal{W}^{\prime}$ into the spaces of doubly left and right regular functions. We conclude that $\left(\rho_{2}^{\prime}, \mathcal{M}\right)$ contains irreducible components isomorphic to $\left(\pi_{d l}, \mathcal{F}^{+}\right),\left(\pi_{d l}, \mathcal{F}^{-}\right),\left(\pi_{d r}, \mathcal{G}^{+}\right)$and $\left(\pi_{d r}, \mathcal{G}^{-}\right)$introduced in Proposition 11]

Recall that we refer to Section 2 of [FL3] for a summary of notations. In particular, we treat $U(2)$ as a subgroup of $\mathbb{H}_{\mathbb{C}}^{\times}$, let $U(2)_{R}=\{R Z ; Z \in U(2)\}$, where $R>0$. We will also need the open domains $\mathbb{D}_{R}^{+}, \mathbb{D}_{R}^{-}$defined by equation (22) in [FL3]. We introduce four maps $p_{a}^{+}, p_{a}^{-}, p_{s}^{+}$ and $p_{s}^{-}: \mathcal{W}^{\prime} \rightarrow \mathcal{W}:$

$$
\begin{aligned}
& p_{a}^{+}: F(Z) \mapsto \frac{i}{2 \pi^{3}}\left[\int_{Z \in U(2)_{R}} \frac{(Z-W)^{-1}}{N(Z-W)} \cdot Z \cdot F(Z)^{+} \frac{d V}{N(Z)}\right]^{+}, \quad W \in \mathbb{D}_{R}^{+}, \\
& p_{a}^{-}: F(Z) \mapsto-\frac{i}{2 \pi^{3}}\left[\int_{Z \in U(2)_{R}} \frac{(Z-W)^{-1}}{N(Z-W)} \cdot Z \cdot F(Z)^{+} \frac{d V}{N(Z)}\right]^{+}, \quad W \in \mathbb{D}_{R}^{-}, \\
& p_{s}^{+}: F(Z) \mapsto \frac{i}{2 \pi^{3}}\left[\int_{Z \in U(2)_{R}} F(Z)^{+} \cdot Z \cdot \frac{(Z-W)^{-1}}{N(Z-W)} \frac{d V}{N(Z)}\right]^{+}, \quad W \in \mathbb{D}_{R}^{+}, \\
& p_{s}^{-}: F(Z) \mapsto-\frac{i}{2 \pi^{3}}\left[\int_{Z \in U(2)_{R}} F(Z)^{+} \cdot Z \cdot \frac{(Z-W)^{-1}}{N(Z-W)} \frac{d V}{N(Z)}\right]^{+}, \quad W \in \mathbb{D}_{R}^{-} .
\end{aligned}
$$

(These maps do not depend on the choice of $R>0$.) First, we show that these maps annihilate the non-harmonic parts.

Lemma 46. Let $F: \mathbb{H}_{\mathbb{C}}^{\times} \rightarrow \mathbb{H}_{\mathbb{C}}$ be such that $\square F=0$ and $k \neq 0$, then

$$
p_{a}^{+}\left(N(Z)^{k} \cdot F(Z)\right)=p_{a}^{-}\left(N(Z)^{k} \cdot F(Z)\right)=p_{s}^{+}\left(N(Z)^{k} \cdot F(Z)\right)=p_{s}^{-}\left(N(Z)^{k} \cdot F(Z)\right)=0 .
$$

In particular, these maps annihilate the non-harmonic parts of functions of the form (38). 
Proof. Observe that

$$
\frac{(Z-W)^{-1}}{N(Z-W)} \cdot Z \quad \text { and } \quad Z \cdot \frac{(Z-W)^{-1}}{N(Z-W)}
$$

are harmonic with respect to the $Z$ variable:

$$
\square_{Z}\left(\frac{(Z-W)^{-1}}{N(Z-W)} \cdot Z\right)=\square_{Z}\left(\frac{(Z-W)^{-1}}{N(Z-W)} \cdot(Z-W)\right)=\square_{Z}\left(\frac{1}{N(Z-W)}\right)=0,
$$

since $\frac{(Z-W)^{-1}}{N(Z-W)}, \frac{(Z-W)^{-1}}{N(Z-W)} \cdot W$ and $\frac{1}{N(Z-W)}$ are harmonic with respect to $Z$. Then the integrals

$$
\begin{aligned}
& \int_{Z \in U(2)_{R}}\left(\frac{(Z-W)^{-1}}{N(Z-W)} \cdot Z\right) \cdot N(Z)^{k-1} \cdot F(Z)^{+} d V=0 \\
& \int_{Z \in U(2)_{R}} N(Z)^{k-1} \cdot F(Z)^{+} \cdot\left(Z \cdot \frac{(Z-W)^{-1}}{N(Z-W)}\right) d V=0
\end{aligned}
$$

by the orthogonality relations (19) in [FL3], since we never get the power of $N(Z)$ that can potentially result in non-zero integral.

Then we determine the effect of the maps $p_{a}^{ \pm}$and $p_{s}^{ \pm}$on harmonic functions. Note that any harmonic function in $\mathcal{W}^{\prime}$ is a linear combination of harmonic parts of functions from Proposition 42

Proposition 47. For each $l, l=0, \frac{1}{2}, 1, \frac{3}{2}, \ldots$,

1. The map $p_{a}^{+}$annihilates $\mathcal{M}(-2 l-2)$ and the components of $\mathcal{M}(2 l)$ isomorphic to $V_{l-\frac{1}{2}} \nabla$ $V_{l-\frac{1}{2}}$ and $V_{l+\frac{1}{2}} \otimes V_{l-\frac{1}{2}}$ from Proposition 42; it leaves the components of $\mathcal{M}(2 l)$ isomorphic to $V_{l-\frac{1}{2}} \otimes V_{l+\frac{1}{2}}$ and $\stackrel{V}{l+\frac{1}{2}}_{V_{l+\frac{1}{2}}}$ unchanged.

2. The map $p_{a}^{-}$annihilates $\mathcal{M}(2 l)$ and the components of $\mathcal{M}(-2 l-2)$ isomorphic to $V_{l-\frac{1}{2}} \square$ $V_{l+\frac{1}{2}}$ and $V_{l+\frac{1}{2}} \otimes V_{l+\frac{1}{2}}$; it leaves the components of $\mathcal{M}(-2 l-2)$ isomorphic to $V_{l-\frac{1}{2}} \otimes V_{l-\frac{1}{2}}$ and $V_{l+\frac{1}{2}} \otimes V_{l-\frac{1}{2}}$ unchanged.

3. The map $p_{s}^{+}$annihilates $\mathcal{M}(-2 l-2)$ and the components of $\mathcal{M}(2 l)$ isomorphic to $V_{l-\frac{1}{2}} \square$ $V_{l-\frac{1}{2}}$ and $V_{l-\frac{1}{2}} \otimes V_{l+\frac{1}{2}}$; it leaves the components of $\mathcal{M}(2 l)$ isomorphic to $V_{l+\frac{1}{2}} \otimes V_{l-\frac{1}{2}}$ and $V_{l+\frac{1}{2}} \otimes V_{l+\frac{1}{2}}$ unchanged.

4. The map $p_{s}^{-}$annihilates $\mathcal{M}(2 l)$ and the components of $\mathcal{M}(-2 l-2)$ isomorphic to $V_{l+\frac{1}{2}} \square$ $V_{l-\frac{1}{2}}$ and $V_{l+\frac{1}{2}} \otimes V_{l+\frac{1}{2}}$; it leaves the components of $\mathcal{M}(-2 l-2)$ isomorphic to $V_{l-\frac{1}{2}} \otimes V_{l-\frac{1}{2}}$ and $V_{l-\frac{1}{2}} \otimes V_{l+\frac{1}{2}}$ unchanged.

5. The component $\mathcal{M}(-1)$ is annihilated by all four maps $p_{a}^{ \pm}, p_{s}^{ \pm}$.

Proof. We show calculations for $p_{a}^{+}$acting on the harmonic part of the component $\mathcal{M}(2 l)$ isomorphic to $V_{l-\frac{1}{2}} \otimes V_{l+\frac{1}{2}}$. Suppose $W \in \mathbb{D}_{R}^{+}$, using Lemma 23 and Proposition 26 from [FL1] 
(see also Proposition 113) we can rewrite the integrand as

$$
\begin{aligned}
& \frac{(Z-W)^{-1}}{N(Z-W)} \cdot Z \cdot F(Z)^{+}
\end{aligned}
$$

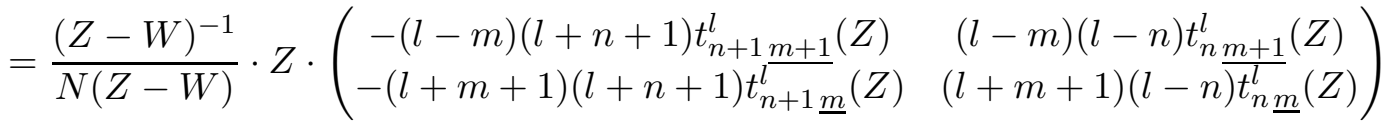

$$
\begin{aligned}
& =\frac{1}{N(Z)} \sum_{l^{\prime}, m^{\prime}, n^{\prime}}\left(\begin{array}{l}
\left(l^{\prime}-m^{\prime}+\frac{1}{2}\right) t_{n^{\prime} l^{\prime}+\frac{1}{2}}^{l^{\prime}}(W) \\
\left(l^{\prime}+m^{\prime}+\frac{1}{2}\right) t_{n^{\prime} \underline{m^{\prime}-\frac{1}{2}}}^{l^{\prime}}(W)
\end{array}\right) \cdot\left(t_{m^{\prime} \underline{n^{\prime}-\frac{1}{2}}}^{l^{\prime}+\frac{1}{2}}\left(Z^{-1}\right), t_{m^{\prime} \underline{n^{\prime}+\frac{1}{2}}}^{l^{\prime}+\frac{1}{2}}\left(Z^{-1}\right)\right)
\end{aligned}
$$

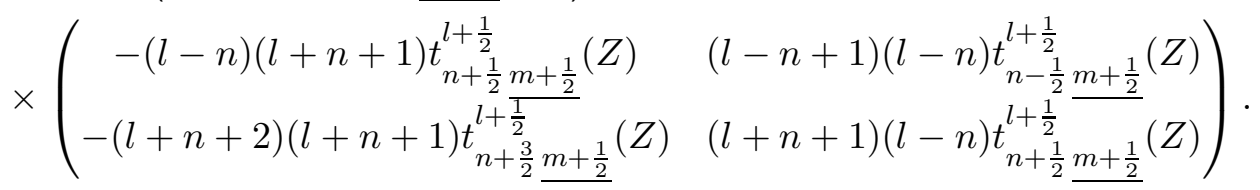

When integrated over $Z \in U(2)_{R}$, by the orthogonality relations (19) in [FL3], only two terms survive - with $l^{\prime}=l, m^{\prime}=m+\frac{1}{2}$ and $n^{\prime}=n$ or $n+1$ :

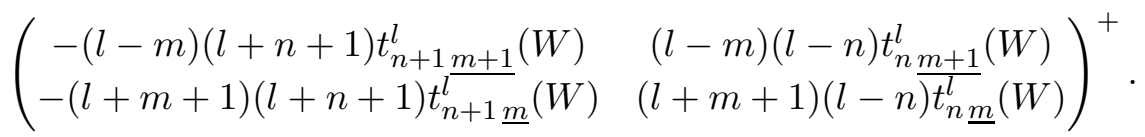

The other cases are similar.

We introduce $\mathbb{C}$-linear maps $\mathbb{S} \rightarrow \mathbb{S}^{\prime}$ and $\mathbb{S}^{\prime} \rightarrow \mathbb{S}$ :

$$
\left(\begin{array}{l}
s_{1} \\
s_{2}
\end{array}\right)^{\dagger}=\left(s_{2},-s_{1}\right), \quad\left(s_{1}^{\prime}, s_{2}^{\prime}\right)^{\dagger}=\left(\begin{array}{c}
-s_{2}^{\prime} \\
s_{1}^{\prime}
\end{array}\right), \quad\left(\begin{array}{c}
s_{1} \\
s_{2}
\end{array}\right) \in \mathbb{S},\left(s_{1}^{\prime}, s_{2}^{\prime}\right) \in \mathbb{S}^{\prime} .
$$

These maps are similar to quaternionic conjugation:

$$
\left(s^{\dagger}\right)^{\dagger}=s, \quad\left(s^{\prime \dagger}\right)^{\dagger}=s^{\prime}, \quad(Z s)^{\dagger}=s^{\dagger} Z^{+}, \quad\left(s^{\prime} Z\right)^{\dagger}=Z^{+} s^{\prime \dagger},
$$

for all $Z \in \mathbb{H}_{\mathbb{C}}, s \in \mathbb{S}, s^{\prime} \in \mathbb{S}^{\prime}$. Then we have two more isomorphisms induced by these maps:

$$
\begin{aligned}
\sigma: \mathbb{H}_{\mathbb{C}} & \simeq \mathbb{S} \otimes \mathbb{S}^{\prime} \rightarrow \mathbb{S} \otimes \mathbb{S}, \quad s \otimes s^{\prime} \mapsto s \otimes s^{\prime \dagger}, \\
\sigma^{\prime}: \mathbb{H}_{\mathbb{C}} & \simeq \mathbb{S} \otimes \mathbb{S}^{\prime} \rightarrow \mathbb{S}^{\prime} \otimes \mathbb{S}^{\prime}, \quad s \otimes s^{\prime} \mapsto s^{\dagger} \otimes s^{\prime}
\end{aligned}
$$

If we identify $\mathbb{S} \otimes \mathbb{S}$ with 4 -columns and $\mathbb{S}^{\prime} \otimes \mathbb{S}^{\prime}$ with 4-rows as in (18), the maps $\sigma$ and $\sigma^{\prime}$ can be expressed as

$$
\sigma\left(\begin{array}{ll}
z_{11} & z_{12} \\
z_{21} & z_{22}
\end{array}\right)=\left(\begin{array}{c}
z_{12} \\
-z_{11} \\
z_{22} \\
-z_{21}
\end{array}\right), \quad \sigma^{\prime}\left(\begin{array}{ll}
z_{11} & z_{12} \\
z_{21} & z_{22}
\end{array}\right)=\left(-z_{21},-z_{22}, z_{11}, z_{12}\right) .
$$

Note that $\amalg I \otimes \mathbb{S} \otimes \mathbb{S}$ and $\Pi I \otimes \mathbb{S}^{\prime} \otimes \mathbb{S}^{\prime}$ denote respectively $\mathbb{S} \otimes \mathbb{S}$ and $\mathbb{S}^{\prime} \otimes \mathbb{S}^{\prime}$-valued polynomial functions on $\mathbb{H}_{\mathbb{C}}^{\times}$. The maps $\sigma$ and $\sigma^{\prime}$ naturally extend to maps

$$
\sigma: \mathcal{W} \rightarrow H \otimes \mathbb{S} \otimes \mathbb{S} \quad \text { and } \quad \sigma^{\prime}: \mathcal{W} \rightarrow \amalg I \mathbb{S}^{\prime} \otimes \mathbb{S}^{\prime} .
$$

We are particularly interested in the following four maps:

$$
\tau_{a}^{+}, \tau_{a}^{-}: \mathcal{W}^{\prime} \rightarrow \amalg \otimes \mathbb{S} \otimes \mathbb{S}, \quad \tau_{s}^{+}, \tau_{s}^{-}: \mathcal{W}^{\prime} \rightarrow \amalg \otimes \mathbb{S}^{\prime} \otimes \mathbb{S}^{\prime}:
$$




$$
\begin{aligned}
& \tau_{a}^{+}(F)=-\sigma\left(\partial\left(p_{a}^{+}(F)\right)\right), \quad \tau_{s}^{+}(F)=\sigma^{\prime}\left(\left(p_{s}^{+}(F)\right) \overleftarrow{\partial}\right) \\
& \tau_{a}^{-}(F)=-\sigma\left(\partial\left(p_{a}^{-}(F)\right)\right), \quad \tau_{s}^{-}(F)=\sigma^{\prime}\left(\left(p_{s}^{-}(F)\right) \overleftarrow{\partial}\right)
\end{aligned}
$$

Combining Lemmas 45, 46 and Proposition 47, we obtain the following description of these maps:

Lemma 48. The maps $\tau_{a}^{ \pm}$and $\tau_{s}^{ \pm}$annihilate all functions of the form $N(Z)^{k} \cdot F(Z)$ with $F: \mathbb{H}_{\mathbb{C}}^{\times} \rightarrow \mathbb{H}_{\mathbb{C}}$ harmonic and $k \neq 0$. For each $l, l=\frac{1}{2}, 1, \frac{3}{2}, 2, \ldots$,

1. $\tau_{a}^{+}$annihilates $\mathcal{M}(-2 l-2)$, projects $\mathcal{M}(2 l)$ onto the $V_{l-\frac{1}{2}} \otimes V_{l+\frac{1}{2}}$ component in the decomposition (41), then applies $\partial$ and identifies the result with a polynomial function $\mathbb{H}_{\mathbb{C}}^{\times} \rightarrow \mathbb{S} \otimes \mathbb{S}$;

2. $\tau_{a}^{-}$annihilates $\mathcal{M}(2 l)$, projects $\mathcal{M}(-2 l-2)$ onto the $V_{l+\frac{1}{2}} \otimes V_{l-\frac{1}{2}}$ component in the decomposition (42), then applies $\partial$ and identifies the result with a polynomial function $\mathbb{H}_{\mathbb{C}}^{\times} \rightarrow \mathbb{S} \otimes \mathbb{S}$;

3. $\tau_{s}^{+}$annihilates $\mathcal{M}(-2 l-2)$, projects $\mathcal{M}(2 l)$ onto the $V_{l+\frac{1}{2}} \otimes V_{l-\frac{1}{2}}$ component in the decomposition (41), then applies $\partial$ on the right and identifies the result with a polynomial function $\mathbb{H}_{\mathbb{C}}^{\times} \rightarrow \mathbb{S}^{\prime} \otimes \mathbb{S}^{\prime}$

4. $\tau_{s}^{-}$annihilates $\mathcal{M}(2 l)$, projects $\mathcal{M}(-2 l-2)$ onto the $V_{l-\frac{1}{2}} \otimes V_{l+\frac{1}{2}}$ component in the decomposition (42), then applies $\partial$ on the right and identifies the result with a polynomial function $\mathbb{H}_{\mathbb{C}}^{\times} \rightarrow \mathbb{S}^{\prime} \otimes \mathbb{S}^{\prime}$

5. The component $\mathcal{M}(-1)$ is annihilated by all four maps $\tau_{a}^{ \pm}, \tau_{s}^{ \pm}$.

Theorem 49. For each $F \in \mathcal{W}^{\prime}, \tau_{a}^{+}(F)$ and $\tau_{a}^{-}(F)$ are polynomial functions $\mathbb{H}_{\mathbb{C}}^{\times} \rightarrow \mathbb{S} \odot \mathbb{S}$ that are doubly left regular, and $\tau_{s}^{+}(F)$ and $\tau_{s}^{-}(F)$ are polynomial functions $\mathbb{H}_{\mathbb{C}}^{\times} \rightarrow \mathbb{S}^{\prime} \odot \mathbb{S}^{\prime}$ that are doubly right regular.

Moreover, the maps $\tau_{a}^{ \pm}, \tau_{s}^{ \pm}$produce isomorphisms of representations of $\mathfrak{s l}\left(2, \mathbb{H}_{\mathbb{C}}\right)$ :

$$
\begin{array}{ll}
\tau_{a}^{+}:\left(\rho_{2}^{\prime}, \mathcal{W}^{\prime} / \operatorname{ker} \tau_{a}^{+}\right) \simeq\left(\pi_{d l}, \mathcal{F}^{+}\right), & \tau_{a}^{-}:\left(\rho_{2}^{\prime}, \mathcal{W}^{\prime} / \operatorname{ker} \tau_{a}^{-}\right) \simeq\left(\pi_{d l}, \mathcal{F}^{-}\right), \\
\tau_{s}^{+}:\left(\rho_{2}^{\prime}, \mathcal{W}^{\prime} / \operatorname{ker} \tau_{s}^{+}\right) \simeq\left(\pi_{d r}, \mathcal{G}^{+}\right), & \tau_{s}^{-}:\left(\rho_{2}^{\prime}, \mathcal{W}^{\prime} / \operatorname{ker} \tau_{s}^{-}\right) \simeq\left(\pi_{d r}, \mathcal{G}^{-}\right) .
\end{array}
$$

(Recall that the spaces $\mathcal{F}^{ \pm}$and $\mathcal{G}^{ \pm}$were introduced in equations (21)-(22).)

Remark 50. The maps $\tau_{a}^{ \pm}, \tau_{s}^{ \pm}$do not produce isomorphisms of representations of $\mathfrak{g l}\left(2, \mathbb{H}_{\mathbb{C}}\right)$ because the scalar matrices act trivially via the $\rho_{2}^{\prime}$ action and non-trivially via the $\pi_{d l}$ and $\pi_{d r}$ actions.

Proof. By direct computation, using Lemmas 45, 48, identity (37) and Lemma 22 from [FL1],

$$
\begin{aligned}
& \tau_{a}^{+}\left(\tilde{F}_{l, m, n}(Z)\right)=(2 l+1) F_{l-\frac{1}{2}, m+\frac{1}{2}, n+\frac{1}{2}}(Z), \\
& \tau_{a}^{-}\left(\tilde{F}_{l, m, n}^{\prime}(Z)\right)=-(2 l+1) F_{l-\frac{1}{2}, m+\frac{1}{2}, n+\frac{1}{2}}^{\prime}(Z), \\
& \tau_{s}^{+}\left(\tilde{G}_{l, m, n}(Z)\right)=(2 l+1) G_{l-\frac{1}{2}, n+\frac{1}{2}, m+\frac{1}{2}}(Z), \\
& \tau_{s}^{-}\left(\tilde{G}_{l, m, n}^{\prime}(Z)\right)=-(2 l+1) G_{l-\frac{1}{2}, n+\frac{1}{2}, m+\frac{1}{2}}^{\prime}(Z) \text {; } \\
& \left(\begin{array}{cc}
\partial_{11} \tilde{F}_{l, m, n}(Z) & \partial_{21} \tilde{F}_{l, m, n}(Z) \\
\partial_{12} \tilde{F}_{l, m, n}(Z) & \partial_{22} \tilde{F}_{l, m, n}(Z)
\end{array}\right)=\frac{2 l+1}{2 l}\left(\begin{array}{cc}
(l-m) \tilde{F}_{l-\frac{1}{2}, m+\frac{1}{2}, n+\frac{1}{2}}(Z) & (l-m) \tilde{F}_{l-\frac{1}{2}, m+\frac{1}{2}, n-\frac{1}{2}}(Z) \\
(l+m+1) \tilde{F}_{l-\frac{1}{2}, m-\frac{1}{2}, n+\frac{1}{2}}(Z) & (l+m+1) \tilde{F}_{l-\frac{1}{2}, m-\frac{1}{2}, n-\frac{1}{2}}(Z)
\end{array}\right) \\
& +\frac{1}{2 l}\left(\begin{array}{cc}
(l-m)(l+n+1) \tilde{H}_{l-\frac{1}{2}, m+\frac{1}{2}, n+\frac{1}{2}}(Z) & -(l-m)(l-n) \tilde{H}_{l-\frac{1}{2}, m+\frac{1}{2}, n-\frac{1}{2}}(Z) \\
(l+m+1)(l+n+1) \tilde{H}_{l-\frac{1}{2}, m-\frac{1}{2}, n+\frac{1}{2}}(Z) & -(l+m+1)(l-n) \tilde{H}_{l-\frac{1}{2}, m-\frac{1}{2}, n-\frac{1}{2}}(Z)
\end{array}\right),
\end{aligned}
$$




$$
\begin{aligned}
& \left(\begin{array}{cc}
\partial_{11} \tilde{F}_{l, m, n}^{\prime}(Z) & \partial_{21} \tilde{F}_{l, m, n}^{\prime}(Z) \\
\partial_{12} \tilde{F}_{l, m, n}^{\prime}(Z) & \partial_{22} \tilde{F}_{l, m, n}^{\prime}(Z)
\end{array}\right)=-\frac{2 l+1}{2 l+2}\left(\begin{array}{cc}
(l-n) \tilde{F}_{l+\frac{1}{2}, m-\frac{1}{2}, n-\frac{1}{2}}^{\prime}(Z) & (l-n) \tilde{F}_{l+\frac{1}{2}, m+\frac{1}{2}, n-\frac{1}{2}}^{\prime}(Z) \\
(l+n+1) \tilde{F}_{l+\frac{1}{2}, m-\frac{1}{2}, n+\frac{1}{2}}^{\prime}(Z) & (l+n+1) \tilde{F}_{l+\frac{1}{2}, m+\frac{1}{2}, n+\frac{1}{2}}^{\prime}(Z)
\end{array}\right) \\
& +\frac{1}{2 l+2}\left(\begin{array}{cc}
(l+m+1)(l-n) \tilde{H}_{l+\frac{1}{2}, m-\frac{1}{2}, n-\frac{1}{2}}^{\prime}(Z) & -(l-m)(l-n) \tilde{H}_{l+\frac{1}{2}, m+\frac{1}{2}, n-\frac{1}{2}}^{\prime}(Z) \\
(l+m+1)(l+n+1) \tilde{H}_{l+\frac{1}{2}, m-\frac{1}{2}, n+\frac{1}{2}}^{\prime} & (Z)-(l-m)(l+n+1) \tilde{H}_{l+\frac{1}{2}, m+\frac{1}{2}, n+\frac{1}{2}}^{\prime}(Z)
\end{array}\right), \\
& +\frac{1}{2 l}\left(\begin{array}{cc}
\tilde{H}_{l-\frac{1}{2}, m+\frac{1}{2}, n+\frac{1}{2}}(Z) & \tilde{H}_{l-\frac{1}{2}, m+\frac{1}{2}, n-\frac{1}{2}}(Z) \\
-\tilde{H}_{l-\frac{1}{2}, m-\frac{1}{2}, n+\frac{1}{2}}(Z) & -\tilde{H}_{l-\frac{1}{2}, m-\frac{1}{2}, n-\frac{1}{2}}(Z)
\end{array}\right), \\
& \left(\begin{array}{cc}
\partial_{11} \tilde{G}_{l, m, n}^{\prime}(Z) & \partial_{21} \tilde{G}_{l, m, n}^{\prime}(Z) \\
\partial_{12} \tilde{G}_{l, m, n}^{\prime}(Z) & \partial_{22} \tilde{G}_{l, m, n}^{\prime}(Z)
\end{array}\right)=-\frac{2 l+1}{2 l+2}\left(\begin{array}{c}
(l-n+1) \tilde{G}_{l+\frac{1}{2}, m-\frac{1}{2}, n-\frac{1}{2}}^{\prime}(Z)(l-n+1) \tilde{G}_{l+\frac{1}{2}, m+\frac{1}{2}, n-\frac{1}{2}}^{\prime}(Z) \\
(l+n+2) \tilde{G}_{l+\frac{1}{2}, m-\frac{1}{2}, n+\frac{1}{2}}^{\prime}(Z)(l+n+2) \tilde{G}_{l+\frac{1}{2}, m+\frac{1}{2}, n+\frac{1}{2}}^{\prime}(Z)
\end{array}\right) \\
& +\frac{1}{2 l+2}\left(\begin{array}{cc}
\tilde{H}_{l+\frac{1}{2}, m-\frac{1}{2}, n-\frac{1}{2}}^{\prime}(Z) & \tilde{H}_{l+\frac{1}{2}, m+\frac{1}{2}, n-\frac{1}{2}}^{\prime}(Z) \\
-\tilde{H}_{l+\frac{1}{2}, m-\frac{1}{2}, n+\frac{1}{2}}^{\prime}(Z) & -\tilde{H}_{l+\frac{1}{2}, m+\frac{1}{2}, n+\frac{1}{2}}^{\prime}(Z)
\end{array}\right) \text {; } \\
& \left(\begin{array}{ll}
\partial_{11} F_{l, m, n}(Z) & \partial_{21} F_{l, m, n}(Z) \\
\partial_{12} F_{l, m, n}(Z) & \partial_{22} F_{l, m, n}(Z)
\end{array}\right)=\left(\begin{array}{cc}
(l-m+1) F_{l-\frac{1}{2}, m+\frac{1}{2}, n+\frac{1}{2}}(Z) & (l-m+1) F_{l-\frac{1}{2}, m+\frac{1}{2}, n-\frac{1}{2}}(Z) \\
(l+m+1) F_{l-\frac{1}{2}, m-\frac{1}{2}, n+\frac{1}{2}}(Z) & (l+m+1) F_{l-\frac{1}{2}, m-\frac{1}{2}, n-\frac{1}{2}}(Z)
\end{array}\right), \\
& \left(\begin{array}{ll}
\partial_{11} F_{l, m, n}^{\prime}(Z) & \partial_{21} F_{l, m, n}^{\prime}(Z) \\
\partial_{12} F_{l, m, n}^{\prime}(Z) & \partial_{22} F_{l, m, n}^{\prime}(Z)
\end{array}\right)=-\left(\begin{array}{ll}
(l-n+1) F_{l+\frac{1}{2}, m-\frac{1}{2}, n-\frac{1}{2}}^{\prime}(Z) & (l-n+1) F_{l+\frac{1}{2}, m+\frac{1}{2}, n-\frac{1}{2}}^{\prime}(Z) \\
(l+n+1) F_{l+\frac{1}{2}, m-\frac{1}{2}, n+\frac{1}{2}}^{\prime}(Z) & (l+n+1) F_{l+\frac{1}{2}, m+\frac{1}{2}, n+\frac{1}{2}}^{\prime}(Z)
\end{array}\right), \\
& \left(\begin{array}{cc}
\partial_{11} G_{l, m, n}(Z) & \partial_{21} G_{l, m, n}(Z) \\
\partial_{12} G_{l, m, n}(Z) & \partial_{22} G_{l, m, n}(Z)
\end{array}\right)=\left(\begin{array}{cc}
(l-n) G_{l-\frac{1}{2}, m+\frac{1}{2}, n+\frac{1}{2}}(Z) & (l-n) G_{l-\frac{1}{2}, m-\frac{1}{2}, n+\frac{1}{2}}(Z) \\
(l+n) G_{l-\frac{1}{2}, m+\frac{1}{2}, n-\frac{1}{2}}(Z) & (l+n) G_{l-\frac{1}{2}, m-\frac{1}{2}, n-\frac{1}{2}}(Z)
\end{array}\right), \\
& \left(\begin{array}{ll}
\partial_{11} G_{l, m, n}^{\prime}(Z) & \partial_{21} G_{l, m, n}^{\prime}(Z) \\
\partial_{12} G_{l, m, n}^{\prime}(Z) & \partial_{22} G_{l, m, n}^{\prime}(Z)
\end{array}\right)=-\left(\begin{array}{cc}
(l-m+2) G_{l+\frac{1}{2}, m-\frac{1}{2}, n-\frac{1}{2}}^{\prime}(Z) & (l-m+2) G_{l+\frac{1}{2}, m-\frac{1}{2}, n+\frac{1}{2}}^{\prime}(Z) \\
(l+m+2) G_{l+\frac{1}{2}, m+\frac{1}{2}, n-\frac{1}{2}}^{\prime}(Z) & (l+m+2) G_{l+\frac{1}{2}, m+\frac{1}{2}, n+\frac{1}{2}}^{\prime}(Z)
\end{array}\right) .
\end{aligned}
$$

It follows from Lemmas 4 and 26 that, if $B \in \mathbb{H}_{\mathbb{C}}$,

$$
\begin{aligned}
\tau_{a}^{+} \circ \rho_{2}^{\prime}\left(\begin{array}{ll}
0 & B \\
0 & 0
\end{array}\right) \tilde{F}_{l, m, n}(Z) & =\pi_{d l}\left(\begin{array}{ll}
0 & B \\
0 & 0
\end{array}\right) \circ \tau_{a}^{+} \tilde{F}_{l, m, n}(Z), \\
\tau_{a}^{-} \circ \rho_{2}^{\prime}\left(\begin{array}{ll}
0 & B \\
0 & 0
\end{array}\right) \tilde{F}_{l, m, n}^{\prime}(Z) & =\pi_{d l}\left(\begin{array}{ll}
0 & B \\
0 & 0
\end{array}\right) \circ \tau_{a}^{-} \tilde{F}_{l, m, n}^{\prime}(Z), \\
\tau_{s}^{+} \circ \rho_{2}^{\prime}\left(\begin{array}{ll}
0 & B \\
0 & 0
\end{array}\right) \tilde{G}_{l, m, n}(Z) & =\pi_{d r}\left(\begin{array}{ll}
0 & B \\
0 & 0
\end{array}\right) \circ \tau_{s}^{+} \tilde{G}_{l, m, n}(Z), \\
\tau_{s}^{-} \circ \rho_{2}^{\prime}\left(\begin{array}{ll}
0 & B \\
0 & 0
\end{array}\right) \tilde{G}_{l, m, n}^{\prime}(Z) & =\pi_{d r}\left(\begin{array}{ll}
0 & B \\
0 & 0
\end{array}\right) \circ \tau_{s}^{-} \tilde{G}_{l, m, n}^{\prime}(Z) .
\end{aligned}
$$

Let $\left(\begin{array}{ll}0 & 1 \\ 1 & 0\end{array}\right) \in G L\left(2, \mathbb{H}_{\mathbb{C}}\right)$, then

$$
\begin{array}{rlrl}
\rho_{2}^{\prime}\left(\begin{array}{ll}
0 & 1 \\
1 & 0
\end{array}\right) \tilde{F}_{l, m, n}(Z)=\tilde{F}_{l, m, n}^{\prime}(Z), & \rho_{2}^{\prime}\left(\begin{array}{ll}
0 & 1 \\
1 & 0
\end{array}\right) \tilde{G}_{l, m, n}(Z)=\tilde{G}_{l, m, n}^{\prime}(Z) ; \\
\pi_{d l}\left(\begin{array}{ll}
0 & 1 \\
1 & 0
\end{array}\right) F_{l, m, n}(Z)=F_{l, m, n}^{\prime}(Z), & & \pi_{d r}\left(\begin{array}{ll}
0 & 1 \\
1 & 0
\end{array}\right) G_{l, m, n}(Z)=G_{l, m, n}^{\prime}(Z) .
\end{array}
$$

This implies that the maps $\tau_{a}^{ \pm}, \tau_{s}^{ \pm}$commute with actions of $\left(\begin{array}{cc}0 & 0 \\ C & 0\end{array}\right), C \in \mathbb{H}_{\mathbb{C}}$. Since matrices of the form $\left(\begin{array}{ll}0 & B \\ 0 & 0\end{array}\right)$ and $\left(\begin{array}{ll}0 & 0 \\ C & 0\end{array}\right), B, C \in \mathbb{H}_{\mathbb{C}}$, generate $\mathfrak{s l}\left(2, \mathbb{H}_{\mathbb{C}}\right)$, the maps $\tau_{a}^{ \pm}, \tau_{s}^{ \pm}$are $\mathfrak{s l}\left(2, \mathbb{H}_{\mathbb{C}}\right)$ equivariant.

Corollary 51. The following representations

$$
\begin{aligned}
& \left(\rho_{2}^{\prime}, \mathcal{W}^{\prime} / \operatorname{ker} \tau_{a}^{+}\right)=\left(\rho_{2}^{\prime}, \mathcal{M} /\left(\operatorname{ker} \tau_{a}^{+} \cap \mathcal{M}\right)\right) \simeq\left(\pi_{d l}, \mathcal{F}^{+}\right) \\
& \left(\rho_{2}^{\prime}, \mathcal{W}^{\prime} / \operatorname{ker} \tau_{a}^{-}\right)=\left(\rho_{2}^{\prime}, \mathcal{M} /\left(\operatorname{ker} \tau_{a}^{-} \cap \mathcal{M}\right)\right) \simeq\left(\pi_{d l}, \mathcal{F}^{-}\right) \\
& \left(\rho_{2}^{\prime}, \mathcal{W}^{\prime} / \operatorname{ker} \tau_{s}^{+}\right)=\left(\rho_{2}^{\prime}, \mathcal{M} /\left(\operatorname{ker} \tau_{s}^{+} \cap \mathcal{M}\right)\right) \simeq\left(\pi_{d r}, \mathcal{G}^{+}\right) \\
& \left(\rho_{2}^{\prime}, \mathcal{W}^{\prime} / \operatorname{ker} \tau_{s}^{-}\right)=\left(\rho_{2}^{\prime}, \mathcal{M} /\left(\operatorname{ker} \tau_{s}^{-} \cap \mathcal{M}\right)\right) \simeq\left(\pi_{d r}, \mathcal{G}^{-}\right)
\end{aligned}
$$

of $\mathfrak{s l}\left(2, \mathbb{H}_{\mathbb{C}}\right)$ are irreducible. 
Proof. By Lemma 48,

$$
\mathcal{W}^{\prime} / \operatorname{ker} \tau_{a}^{+}=\bigoplus_{l=\frac{1}{2}, 1, \frac{3}{2}, 2, \ldots} V_{l-\frac{1}{2}} \otimes V_{l+\frac{1}{2}}
$$

as a representation of $S U(2) \times S U(2)$, where each direct summand $V_{l-\frac{1}{2}} \otimes V_{l+\frac{1}{2}}$ is spanned by $\tilde{F}_{l, m, n}$ 's with $-l-1 \leq m \leq l,-l \leq n \leq l-1$. And by Lemma 26 and equations (47), (48), (49) we have:

$$
\begin{gathered}
\rho_{2}^{\prime}\left(\begin{array}{ll}
0 & B \\
0 & 0
\end{array}\right) \tilde{F}_{l, m, n}(Z)=-\frac{2 l+1}{2 l} \operatorname{Tr}\left[B\left(\begin{array}{cc}
(l-m) \tilde{F}_{l-\frac{1}{2}, m+\frac{1}{2}, n+\frac{1}{2}}(Z) & (l-m) \tilde{F}_{l-\frac{1}{2}, m+\frac{1}{2}, n-\frac{1}{2}}(Z) \\
(l+m+1) \tilde{F}_{l-\frac{1}{2}, m-\frac{1}{2}, n+\frac{1}{2}}(Z) & (l+m+1) \tilde{F}_{l-\frac{1}{2}, m-\frac{1}{2}, n-\frac{1}{2}}(Z)
\end{array}\right)\right], \\
\rho_{2}^{\prime}\left(\begin{array}{ll}
0 & 0 \\
C & 0
\end{array}\right) \tilde{F}_{l, m, n}(Z)=\frac{2 l+1}{2 l+2} \operatorname{Tr}\left[C\left(\begin{array}{cc}
(l-n) \tilde{F}_{l+\frac{1}{2}, m-\frac{1}{2}, n-\frac{1}{2}}(Z) & (l-n) \tilde{F}_{l+\frac{1}{2}, m+\frac{1}{2}, n-\frac{1}{2}}(Z) \\
(l+n+1) \tilde{F}_{l+\frac{1}{2}, m-\frac{1}{2}, n+\frac{1}{2}}(Z) & (l+n+1) \tilde{F}_{l+\frac{1}{2}, m+\frac{1}{2}, n+\frac{1}{2}}(Z)
\end{array}\right)\right]
\end{gathered}
$$

in the quotient space $\mathcal{W}^{\prime} / \operatorname{ker} \tau_{a}^{+}$. It follows that any non-zero vector in $\mathcal{W}^{\prime} / \operatorname{ker} \tau_{a}^{+}$generates the $V_{0} \otimes V_{1}$ component and hence the whole space, thus proving irreducibility of $\left(\rho_{2}^{\prime}, \mathcal{W}^{\prime} / \operatorname{ker} \tau_{a}^{+}\right) \simeq$ $\left(\pi_{d l}, \mathcal{F}^{+}\right)$. The other cases are similar.

\subsection{Quaternionic Analogue of Cauchy's Integral Formula for the Second Or- der Pole}

Note that the maps $\tau_{a}^{ \pm}$and $\tau_{s}^{ \pm}$on $\mathcal{W}^{\prime}$ are given by integrating over a four-dimensional cycle $U(2)_{R}$. For example,

$$
\left(\tau_{a}^{+} F\right)(W)=-\sigma \circ \partial_{W}\left(\frac{i}{2 \pi^{3}} \int_{Z \in U(2)_{R}} F(Z) \cdot Z^{+} \cdot\left[\frac{(Z-W)^{-1}}{N(Z-W)}\right]^{+} \frac{d V}{N(Z)}\right), \quad W \in \mathbb{D}_{R}^{+} .
$$

When restricted to $\mathcal{M} \subset \mathcal{W}^{\prime}$, these maps can be rewritten so that the integral is over the sphere $S_{R}^{3}$ of radius $R$.

Theorem 52. The restrictions of $\tau_{a}^{ \pm}$and $\tau_{s}^{ \pm}$to $\mathcal{M}$ can be rewritten as

$$
\begin{array}{ll}
\left(\tau_{a}^{+} F\right)(W)=-\sigma \circ \partial_{W}\left[\frac{1}{2 \pi^{2}} \int_{Z \in S_{R}^{3}} \frac{(Z-W)^{-1}}{N(Z-W)} \cdot D z \cdot F^{+}(Z)\right]^{+}, & W \in \mathbb{D}_{R}^{+}, \\
\left(\tau_{a}^{-} F\right)(W)=-\sigma \circ \partial_{W}\left[\frac{-1}{2 \pi^{2}} \int_{Z \in S_{R}^{3}} \frac{(Z-W)^{-1}}{N(Z-W)} \cdot D z \cdot F^{+}(Z)\right]^{+}, & W \in \mathbb{D}_{R}^{-} \\
\left(\tau_{s}^{+} F\right)(W)=\sigma^{\prime}\left(\left[\frac{1}{2 \pi^{2}} \int_{Z \in S_{R}^{3}} F^{+}(Z) \cdot D z \cdot \frac{(Z-W)^{-1}}{N(Z-W)}\right]^{+} \overleftarrow{\partial}_{W}\right), & W \in \mathbb{D}_{R}^{+} \\
\left(\tau_{s}^{-} F\right)(W)=\sigma^{\prime}\left(\left[\frac{1}{2 \pi^{2}} \int_{Z \in S_{R}^{3}} F^{+}(Z) \cdot D z \cdot \frac{(Z-W)^{-1}}{N(Z-W)}\right]^{+} \overleftarrow{\partial}_{W}\right), & W \in \mathbb{D}_{R}^{-}
\end{array}
$$

where $F \in \mathcal{M}$.

Remark 53. Note that (apart from quaternionic conjugation), the maps $\tau_{a}^{ \pm}$and $\tau_{s}^{ \pm}$restricted to $\mathcal{M}$ are essentially derivatives of the classical Cauchy-Fueter formulas for left and right regular functions. Thus $\tau_{a}^{ \pm}$and $\tau_{s}^{ \pm}$can be treated as another analogue of Cauchy's integral formula for the second order pole (5). 
Proof. First, we determine the effect of these maps on the non-harmonic parts of functions of the form (38). Suppose that $F_{d}: \mathbb{H}_{\mathbb{C}}^{\times} \rightarrow \mathbb{H}_{\mathbb{C}}$ be homogeneous of degree $d$ and harmonic, then

$$
\nabla^{+}\left(\partial F_{d}(Z) \partial\right)=\left(\partial F_{d}(Z) \partial\right) \overleftarrow{\nabla}^{+}=0
$$

i.e. the function $\partial F_{d}(Z) \partial$ is both left and right regular. Thus we can apply the Cauchy-Fueter formulas. In the case of $\tau_{a}^{+}$, the integral becomes

$$
\left[\frac{1}{2 \pi^{2}} \int_{Z \in S_{R}^{3}} \frac{(Z-W)^{-1}}{N(Z-W)} \cdot D z \cdot\left(\partial F_{d}(Z) \partial\right)\right]^{+}= \begin{cases}\left(\partial F_{d}(W) \partial\right)^{+} & \text {if } d \geq 0 \\ 0 & \text { if } d<0\end{cases}
$$

Applying $\partial_{W}$ results in zero. The cases of $\tau_{a}^{-}$and $\tau_{s}^{ \pm}$are similar. Using Lemma 6 from [FL1], we replace $\left.D z\right|_{S_{R}^{3}}$ with $R^{-1} Z d S$, where $d S$ is the usual Euclidean volume element on the 3 -sphere $S_{R}^{3}$. Then the proof proceeds exactly as those of Proposition 47 and Theorem 49, except using orthogonality relations (17) from [FL3] instead of (19).

\subsection{Invariant Bilinear Pairing on $\left(\rho_{2}^{\prime}, \mathcal{M}\right)$}

We define a bilinear pairing on $\mathcal{M}$ by

$$
\left\langle F_{1}, F_{2}\right\rangle_{\mathcal{M}}=\frac{1}{2 \pi^{2}} \int_{Z \in S_{R}^{3}} \operatorname{Tr}\left(\widetilde{\operatorname{deg}}^{-1}\left(\left(F_{1} \overleftarrow{\partial}\right)(Z) \cdot Z\right) \cdot\left(Z \cdot\left(\vec{\partial}_{F_{2}}\right)(Z)\right)^{+}\right) \frac{d S}{R}
$$

Note that if $F_{1}$ happens to be $N(Z)^{-1} \cdot Z$, then by Lemma 45

$$
\left(F_{1} \overleftarrow{\partial}\right)(Z) \cdot Z=N(Z)^{-1} \cdot Z
$$

and $\widetilde{\operatorname{deg}}^{-1}\left(\left(F_{1} \overleftarrow{\partial}\right)(Z) \cdot Z\right)$ is undefined. Thus we declare the pairing (50) to be zero on $\mathcal{M}(-1)$ We will see shortly that the pairing does not depend on the choice of $R>0$.

Theorem 54. The bilinear pairing (50) on $\mathcal{M}$ is $\mathfrak{s l}\left(2, \mathbb{H}_{\mathbb{C}}\right)$-invariant and does not depend on the choice of $R>0$. We have the following orthogonality relations:

$$
\left\langle\tilde{G}_{l, m, n}(Z), \tilde{F}_{l^{\prime}, n^{\prime}, m^{\prime}}^{\prime}(Z)\right\rangle_{\mathcal{M}}=-\left\langle\tilde{G}_{l, m, n}^{\prime}(Z), \tilde{F}_{l^{\prime}, n^{\prime}, m^{\prime}}(Z)\right\rangle_{\mathcal{M}}=(2 l+1)^{2} \delta_{l l^{\prime}} \delta_{m m^{\prime}} \delta_{n n^{\prime}}
$$

all other pairing between $\tilde{F}_{l, m, n}(Z), \tilde{F}_{l, m, n}^{\prime}(Z), \tilde{G}_{l, m, n}(Z), \tilde{G}_{l, m, n}^{\prime}(Z), \tilde{H}_{l, m, n}(Z), \tilde{H}_{l, m, n}^{\prime}(Z)$, $\tilde{I}_{l, m, n}(Z), \tilde{I}_{l, m, n}^{\prime}(Z)$ are zero; in particular,

$$
\left\langle\tilde{F}_{l, m, n}(Z), \tilde{G}_{l^{\prime}, n^{\prime}, m^{\prime}}^{\prime}(Z)\right\rangle_{\mathcal{M}}=\left\langle\tilde{F}_{l, m, n}^{\prime}(Z), \tilde{G}_{l^{\prime}, n^{\prime}, m^{\prime}}(Z)\right\rangle_{\mathcal{M}}=0
$$

Proof. First, we check the orthogonality relations; they follow from Lemma 45]and orthogonality relations (17) from [FL3]. The orthogonality relations also imply that the pairing is independent of the choice of $R>0$.

Using Proposition 20 and equations (43)-(46) we can relate the bilinear pairing (50) to the pairing for doubly regular functions (25) as

$$
\left\langle F_{1}, F_{2}\right\rangle_{\mathcal{M}}=\left\langle\tau_{a}^{+}\left(F_{2}\right), \tau_{s}^{-}\left(F_{1}\right)\right\rangle_{\mathcal{D R}}+\left\langle\tau_{a}^{-}\left(F_{2}\right), \tau_{s}^{+}\left(F_{1}\right)\right\rangle_{\mathcal{D R}}
$$

Since the right hand side is $\mathfrak{s l}\left(2, \mathbb{H}_{\mathbb{C}}\right)$-invariant, the pairing $(\underline{50})$ is $\mathfrak{s l}\left(2, \mathbb{H}_{\mathbb{C}}\right)$-invariant as well. 


\section{The Quaternionic Chain Complex and Decomposition of $\left(\rho_{2}, \mathcal{W}\right)$, $\left(\rho_{2}^{\prime}, \mathcal{W}^{\prime}\right)$ into Irreducible Components}

\subsection{Decomposition of $\left(\rho_{2}, \mathcal{W}\right)$}

In this subsection we find explicit decomposition of $\left(\rho_{2}, \mathcal{W}\right)$ into irreducible components. The idea is to use the quaternionic chain complex (29) and deal separately with

$$
\operatorname{ker}\left(\operatorname{Tr} \circ \partial^{+}\right) \subset \mathcal{W} \quad \text { and } \quad \mathcal{W} / \operatorname{ker}\left(\operatorname{Tr} \circ \partial^{+}\right) \subset \amalg I .
$$

We will see that there is a total of thirteen irreducible components with $\operatorname{ker}\left(\operatorname{Tr} \circ \partial^{+}\right)$having eight components and $\mathcal{W} / \operatorname{ker}\left(\operatorname{Tr} \circ \partial^{+}\right)$having five.

Lemma 55. The image of the map $\operatorname{Tr} \circ \partial^{+}: \mathcal{W} \rightarrow$ II from (29) is $\mathcal{I}^{+}+\mathcal{I}^{-}$.

Proof. Indeed, $\operatorname{Tr} \circ \partial^{+}\left(N(Z)^{-1} \cdot Z^{+}\right)=2 N(Z)^{-1}$, which generates $\mathcal{I}^{+}$, and $\operatorname{Tr} \circ \partial^{+}\left(N(Z)^{-3}\right.$. $\left.Z^{+}\right)=-2 N(Z)^{-3}$, which generates $\mathcal{I}^{-}$. Thus the image of $\operatorname{Tr} \circ \partial^{+}$contains $\mathcal{I}^{+}+\mathcal{I}^{-}$. It remains to show that the image is $\mathcal{I}^{+}+\mathcal{I}^{-}$. Otherwise, by Theorem 31, the image of $\operatorname{Tr} \circ \partial^{+}$ is all of $\amalg$. In particular, there exists a homogeneous function $F \in \mathcal{W}$ of degree -3 such that $\operatorname{Tr} \circ \partial^{+} F=N(Z)^{-2}$. Such a function $F$ must be a linear combination of

$$
N(Z)^{-2} \cdot H_{1}(Z), \quad N(Z)^{-3} \cdot H_{3}(Z), \quad N(Z)^{-4} \cdot H_{5}(Z), \ldots
$$

with $H_{1}(Z), H_{3}(Z), H_{5}(Z), \ldots$ harmonic and homogeneous of degrees $1,3,5, \ldots$ respectively. On the other hand, the $\rho$ action of $\mathfrak{s l}(2, \mathbb{C}) \times \mathfrak{s l}(2, \mathbb{C})$ on $N(Z)^{-2}$ is trivial, hence the $\rho_{2}$ action of $\mathfrak{s l}(2, \mathbb{C}) \times \mathfrak{s l}(2, \mathbb{C})$ on $H_{1}(Z), H_{3}(Z), H_{5}(Z), \ldots$ must be trivial as well. Since each $H_{k}(Z)$ lies in $\left(V_{\frac{k}{2}} \otimes V_{\frac{1}{2}}\right) \otimes\left(V_{\frac{k}{2}} \otimes V_{\frac{1}{2}}\right)$, it follows that $H_{3}(Z)=H_{5}(Z)=\cdots=0$ and $H_{1}(Z)$ is proportional to $N(Z)^{-2} \cdot Z^{+}$. But $\operatorname{Tr} \circ \partial^{+}\left(N(Z)^{-2} \cdot Z^{+}\right)=0$, which gives us a contradiction.

Combining this with Theorem 31 we obtain:

Corollary 56. The quotient $\left(\rho_{2}, \mathcal{W} / \operatorname{ker}\left(\operatorname{Tr} \circ \partial^{+}\right)\right)$has five irreducible components that are isomorphic to

$$
\left(\rho, W^{+}\right), \quad\left(\rho, W^{-}\right), \quad(\rho, \mathcal{J}), \quad\left(\rho, \mathcal{I}^{+} /\left(H^{+} \oplus \mathcal{J}\right)\right), \quad\left(\rho, \mathcal{I}^{-} /\left(H^{-} \oplus \mathcal{J}\right)\right) .
$$

Our next task is to decompose $\operatorname{ker}\left(\operatorname{Tr} \circ \partial^{+}\right)$.

Lemma 57. We have:

$$
\begin{aligned}
& \operatorname{Tr} \circ \partial^{+}\left[N(Z)^{k} \cdot\left(\begin{array}{cc}
\alpha_{11} t_{n+1}^{l} \underline{m+1}(Z) & \alpha_{12} t_{n \underline{m+1}}^{l}(Z) \\
\alpha_{21} t_{n+1 \underline{m}}^{l}(Z) & \alpha_{22} t_{n \underline{m}}^{l}(Z)
\end{array}\right)\right] \\
& =\frac{2 l+k+1}{2 l+1}\left((l+m+1)\left(\alpha_{11}-\alpha_{12}\right)+(l-m)\left(\alpha_{22}-\alpha_{21}\right)\right) \cdot N(Z)^{k} \cdot t_{n+\frac{1}{2} \underline{m+\frac{1}{2}}}^{l-\frac{1}{2}}(Z) \\
& +\frac{k}{2 l+1}\left((l-n)\left(\alpha_{11}+\alpha_{21}\right)+(l+n+1)\left(\alpha_{12}+\alpha_{22}\right)\right) \cdot N(Z)^{k-1} \cdot t_{n+\frac{1}{2} \underline{m+\frac{1}{2}}}^{l+\frac{1}{2}}(Z) ; \\
& \operatorname{Tr} \circ \partial^{+}\left[N(Z)^{k-1} \cdot\left(\begin{array}{cc}
\beta_{11} t_{n \underline{m}}^{l}\left(Z^{-1}\right) & \beta_{12} t_{n}^{l} \underline{\underline{m+1}}\left(Z^{-1}\right) \\
\beta_{21} t_{n+1 \underline{m}}^{l}\left(Z^{-1}\right) & \beta_{22} t_{n+1}^{l} \underline{\underline{m+1}}\left(Z^{-1}\right)
\end{array}\right)\right] \\
& =-\frac{2 l-k+1}{2 l+1}\left((l+n+1)\left(\beta_{11}-\beta_{12}\right)+(l-n)\left(\beta_{22}-\beta_{21}\right)\right) \cdot N(Z)^{k-1} \cdot t_{n+\frac{1}{2} \underline{m+\frac{1}{2}}}^{l+\frac{1}{2}}\left(Z^{-1}\right) \\
& +\frac{k}{2 l+1}\left((l-m)\left(\beta_{11}+\beta_{21}\right)+(l+m+1)\left(\beta_{12}+\beta_{22}\right)\right) \cdot N(Z)^{k-2} \cdot t_{n+\frac{1}{2} \underline{m+\frac{1}{2}}}^{l-\frac{1}{2}}\left(Z^{-1}\right) .
\end{aligned}
$$


Proof. The result follows by direct computation using Lemma 22 from [FL1] and identities (34), (37).

Let

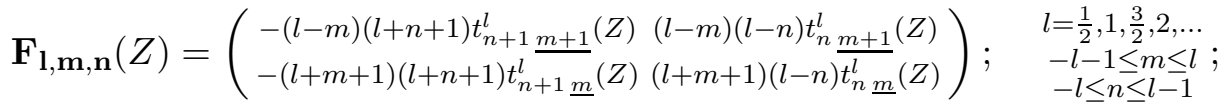

$$
\begin{aligned}
& \mathbf{G}_{\mathbf{l}, \mathbf{m}, \mathbf{n}}(Z)=\left(\begin{array}{cc}
-t_{n+1}^{l} \underline{m+1}(Z) & -t_{n}^{l} \underline{m+1}(Z) \\
t_{n+1}^{l} \underline{\underline{m}}(Z) & t_{n \underline{m}}^{l}(Z)
\end{array}\right) ; \begin{array}{c}
l=\frac{1}{2}, 1, \frac{3}{2}, 2, \ldots \\
-l \leq m \leq l-1 \\
-l-1 \leq n \leq l
\end{array}
\end{aligned}
$$

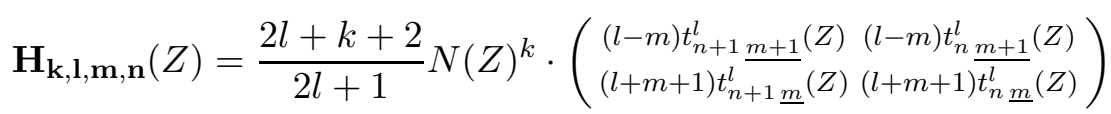

$$
\begin{aligned}
& -\frac{k}{2 l+3} N(Z)^{k-1} \cdot\left(\begin{array}{cc}
(l+n+2) t_{n+1}^{l+1} \frac{m+1}{1+1}(Z) & -(l-n+1) t_{n}^{l+1}(Z+1 \\
-(l+n+2) t_{n+1}^{l+1}(Z) & (l-n+1) t_{n \underline{m}}^{l+1}(Z)
\end{array}\right) ; \begin{array}{c}
k=0, \pm 1, \pm 2, \ldots \\
l=0, \frac{1}{2}, 1, \frac{3}{2}, \ldots \\
-l-1 \leq m, n \leq l
\end{array} .
\end{aligned}
$$

We also introduce

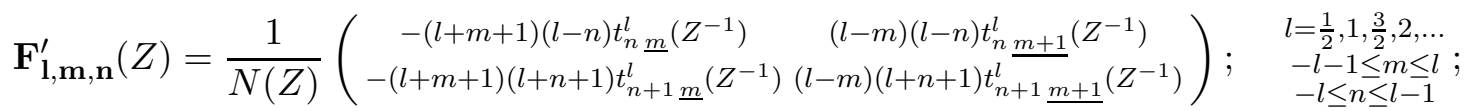

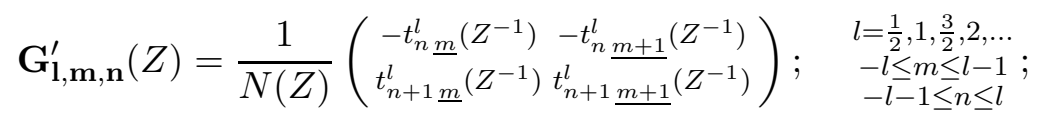

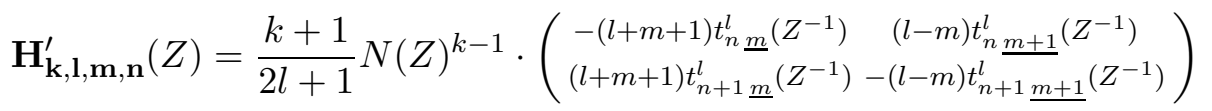

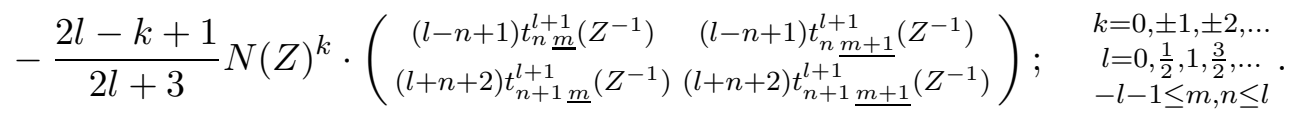

Let $K=U(2) \times U(2)$ sitting as the diagonal subgroup of $G L\left(2, \mathbb{H}_{\mathbb{C}}\right)$.

\section{Proposition 58. The functions}

$$
N(Z)^{k} \cdot \mathbf{F}_{\mathbf{l}, \mathbf{m}, \mathbf{n}}(Z), \quad N(Z)^{k} \cdot \mathbf{G}_{\mathbf{l}, \mathbf{m}, \mathbf{n}}(Z), \quad \mathbf{H}_{\mathbf{k}, \mathbf{l}, \mathbf{m}, \mathbf{n}}(Z) \quad \text { and } \quad N(Z)^{-2} \cdot Z^{+}
$$

span the kernel of $\operatorname{Tr} \circ \partial^{+}: \mathcal{W} \rightarrow \amalg$ and generate the $K$-types of the kernel. More precisely, as representations of $S U(2) \times S U(2)$,

$$
V_{0} \otimes V_{0}=\mathbb{C} \text {-span of }\left\{N(Z)^{-2} \cdot Z^{+}\right\} \text {, }
$$

and, for $k, l$ fixed,

$$
\begin{aligned}
& V_{l-\frac{1}{2}} \otimes V_{l+\frac{1}{2}}=\mathbb{C} \text {-span of }\left\{N(Z)^{k} \cdot \mathbf{F}_{\mathbf{l}, \mathbf{m}, \mathbf{n}}(Z) ; \begin{array}{l}
-l-1 \leq m \leq l \\
-l \leq n \leq l-1
\end{array}\right\}, \\
& V_{l+\frac{1}{2}} \otimes V_{l-\frac{1}{2}}=\mathbb{C} \text {-span of }\left\{N(Z)^{k} \cdot \mathbf{G}_{\mathbf{l}, \mathbf{m}, \mathbf{n}}(Z) ; \begin{array}{l}
-l \leq m \leq l-1 \\
-l-1 \leq n \leq l
\end{array}\right\}, \\
& V_{l+\frac{1}{2}} \otimes V_{l+\frac{1}{2}}=\mathbb{C} \text {-span of }\left\{\mathbf{H}_{\mathbf{k}, \mathbf{l}, \mathbf{m}, \mathbf{n}}(Z) ;-l-1 \leq m, n \leq l\right\} .
\end{aligned}
$$

Proof. Clearly, these functions are $K$-finite, linearly independent and annihilated by $\operatorname{Tr} \circ \partial^{+}$. It remains to show that these functions span all of the kernel of $\operatorname{Tr} \circ \partial^{+}$. This is done by checking, for each $d \in \mathbb{Z}$, that if one takes the $K$-types of

$$
\mathcal{W}(d)=\{F(Z) \in \mathcal{W} ; F(Z) \text { is homogeneous of degree } d\}
$$

and "subtracts" the $K$-types of

$$
\left(\mathcal{I}^{+}+\mathcal{I}^{-}\right)(d-1)=\left\{f(Z) \in \mathcal{I}^{+}+\mathcal{I}^{-} ; f(Z) \text { is homogeneous of degree } d-1\right\}
$$

then all the remaining $K$-types are accounted for in (51). 
We compute the action of $\left(\begin{array}{ll}0 & B \\ 0 & 0\end{array}\right) \in \mathfrak{g l}\left(2, \mathbb{H}_{\mathbb{C}}\right), B \in \mathbb{H}_{\mathbb{C}}$, on these generators:

$$
\begin{aligned}
& \left(\begin{array}{cc}
\partial_{11}\left(N(Z)^{k} \cdot \mathbf{F}_{\mathbf{1 , \mathbf { m } , \mathbf { n }}}(Z)\right) & \partial_{21}\left(N(Z)^{k} \cdot \mathbf{F}_{\mathbf{1 , \mathbf { m } , \mathbf { n }}}(Z)\right) \\
\partial_{12}\left(N(Z)^{k} \cdot \mathbf{F}_{\mathbf{1 , \mathbf { m } , \mathbf { n }}}(Z)\right) & \partial_{22}\left(N(Z)^{k} \cdot \mathbf{F}_{\mathbf{1}, \mathbf{m}, \mathbf{n}}(Z)\right)
\end{array}\right) \\
& =\frac{2 l+k+1}{2 l} N(Z)^{k}\left(\begin{array}{cc}
(l-m) \mathbf{F}_{\mathbf{l}-\frac{1}{2}, \mathbf{m}+\frac{1}{2}, \mathbf{n}+\frac{1}{2}}(Z) & (l-m) \mathbf{F}_{\mathbf{1}-\frac{1}{2}, \mathbf{m}+\frac{1}{2}, \mathbf{n}-\frac{1}{2}}(Z) \\
(l+m+1) \mathbf{F}_{\mathbf{l}-\frac{1}{2}, \mathbf{m}-\frac{1}{2}, \mathbf{n}+\frac{1}{2}}(Z) & (l+m+1) \mathbf{F}_{\mathbf{1}-\frac{1}{2}, \mathbf{m}-\frac{1}{2}, \mathbf{n}-\frac{1}{2}}(Z)
\end{array}\right) \\
& +\frac{k}{2 l+2} N(Z)^{k-1}\left(\begin{array}{cc}
(l+n+1) \mathbf{F}_{\mathbf{l}+\frac{1}{2}, \mathbf{m}+\frac{1}{2}, \mathbf{n}+\frac{1}{2}}(Z) & -(l-n) \mathbf{F}_{\mathbf{l}+\frac{1}{2}, \mathbf{m}+\frac{1}{2}, \mathbf{n}-\frac{1}{2}}(Z) \\
-(l+n+1) \mathbf{F}_{\mathbf{l}+\frac{1}{2}, \mathbf{m}-\frac{1}{2}, \mathbf{n}+\frac{1}{2}}(Z) & (l-n) \mathbf{F}_{\mathbf{l}+\frac{1}{2}, \mathbf{m}-\frac{1}{2}, \mathbf{n}-\frac{1}{2}}(Z)
\end{array}\right) \\
& +\frac{1}{2 l+1}\left(\begin{array}{cc}
(l-m)(l+n+1) \mathbf{H}_{\mathbf{k}, \mathbf{l}-\frac{1}{2}, \mathbf{m}+\frac{1}{2}, \mathbf{n}+\frac{1}{2}}(Z) & -(l-m)(l-n) \mathbf{H}_{\mathbf{k}, \mathbf{l}-\frac{1}{2}, \mathbf{m}+\frac{1}{2}, \mathbf{n}-\frac{1}{2}}(Z) \\
(l+m+1)(l+n+1) \mathbf{H}_{\mathbf{k}, \mathbf{l}-\frac{1}{2}, \mathbf{m}-\frac{1}{2}, \mathbf{n}+\frac{1}{2}}(Z) & -(l+m+1)(l-n) \mathbf{H}_{\mathbf{k}, \mathbf{l}-\frac{1}{2}, \mathbf{m}-\frac{1}{2}, \mathbf{n}-\frac{1}{2}}(Z)
\end{array}\right) ; \\
& \left(\begin{array}{cc}
\partial_{11}\left(N(Z)^{k} \cdot \mathbf{G}_{\mathbf{l}, \mathbf{m}, \mathbf{n}}(Z)\right) & \partial_{21}\left(N(Z)^{k} \cdot \mathbf{G}_{\mathbf{l}, \mathbf{m}, \mathbf{n}}(Z)\right) \\
\partial_{12}\left(N(Z)^{k} \cdot \mathbf{G}_{\mathbf{l}, \mathbf{m}, \mathbf{n}}(Z)\right) & \partial_{22}\left(N(Z)^{k} \cdot \mathbf{G}_{\mathbf{l}, \mathbf{m}, \mathbf{n}}(Z)\right)
\end{array}\right) \\
& =\frac{2 l+k+1}{2 l} N(Z)^{k}\left(\begin{array}{cc}
(l-m-1) \mathbf{G}_{\mathbf{l}-\frac{1}{2}, \mathbf{m}+\frac{1}{2}, \mathbf{n}+\frac{1}{2}}(Z) & (l-m-1) \mathbf{G}_{\mathbf{1}-\frac{1}{2}, \mathbf{m}+\frac{1}{2}, \mathbf{n}-\frac{1}{2}}(Z) \\
(l+m) \mathbf{G}_{\mathbf{1}-\frac{1}{2}, \mathbf{m}-\frac{1}{2}, \mathbf{n}+\frac{1}{2}}(Z) & (l+m) \mathbf{G}_{\mathbf{1}-\frac{1}{2}, \mathbf{m}-\frac{1}{2}, \mathbf{n}-\frac{1}{2}}(Z)
\end{array}\right) \\
& +\frac{k}{2 l+2} N(Z)^{k-1}\left(\begin{array}{cc}
(l+n+2) \mathbf{G}_{\mathbf{l}+\frac{1}{2}, \mathbf{m}+\frac{1}{2}, \mathbf{n}+\frac{1}{2}}(Z) & -(l-n+1) \mathbf{G}_{\mathbf{l}+\frac{1}{2}, \mathbf{m}+\frac{1}{2}, \mathbf{n}-\frac{1}{2}}(Z) \\
-(l+n+2) \mathbf{G}_{\mathbf{l}+\frac{1}{2}, \mathbf{m}-\frac{1}{2}, \mathbf{n}+\frac{1}{2}}(Z) & (l-n+1) \mathbf{G}_{\mathbf{l}+\frac{1}{2}, \mathbf{m}-\frac{1}{2}, \mathbf{n}-\frac{1}{2}}(Z)
\end{array}\right) \\
& +\frac{1}{2 l+1}\left(\begin{array}{cc}
\mathbf{H}_{\mathbf{k}, \mathbf{l}-\frac{1}{2}, \mathbf{m}+\frac{1}{2}, \mathbf{n}+\frac{1}{2}}(Z) & \mathbf{H}_{\mathbf{k}, \mathbf{l}-\frac{1}{2}, \mathbf{m}+\frac{1}{2}, \mathbf{n}-\frac{1}{2}}(Z) \\
-\mathbf{H}_{\mathbf{k}, \mathbf{l}-\frac{1}{2}, \mathbf{m}-\frac{1}{2}, \mathbf{n}+\frac{1}{2}}(Z) & -\mathbf{H}_{\mathbf{k}, \mathbf{l}-\frac{1}{2}, \mathbf{m}-\frac{1}{2}, \mathbf{n}-\frac{1}{2}}(Z)
\end{array}\right) ;
\end{aligned}
$$

$$
\begin{aligned}
& \left(\begin{array}{ll}
\partial_{11} \mathbf{H}_{\mathbf{k}, \mathbf{l}, \mathbf{m}, \mathbf{n}}(Z) & \partial_{21} \mathbf{H}_{\mathbf{k}, \mathbf{l}, \mathbf{m}, \mathbf{n}}(Z) \\
\partial_{12} \mathbf{H}_{\mathbf{k}, \mathbf{l}, \mathbf{m}, \mathbf{n}}(Z) & \partial_{22} \mathbf{H}_{\mathbf{k}, \mathbf{l}, \mathbf{m}, \mathbf{n}}(Z)
\end{array}\right) \\
& =\frac{k(2 l+k+2) N(Z)^{k-1}}{(l+1)(2 l+1)(2 l+3)}\left(\begin{array}{cc}
-\mathbf{F}_{\mathbf{1}+\frac{1}{2}, \mathbf{m}+\frac{1}{2}, \mathbf{n}+\frac{1}{2}}(Z) & -\mathbf{F}_{\mathbf{1}+\frac{1}{2}, \mathbf{m}+\frac{1}{2}, \mathbf{n}-\frac{1}{2}}(Z) \\
\mathbf{F}_{\mathbf{1 + \frac { 1 } { 2 }}, \mathbf{m}-\frac{1}{2}, \mathbf{n}+\frac{1}{2}}(Z) & \mathbf{F}_{\mathbf{1 + \frac { 1 } { 2 }}, \mathbf{m}-\frac{1}{2}, \mathbf{n}-\frac{1}{2}}(Z)
\end{array}\right) \\
& +\frac{k(2 l+k+2) N(Z)^{k-1}}{(l+1)(2 l+1)(2 l+3)} \\
& \times\left(\begin{array}{cc}
-(l-m)(l+n+2) \mathbf{G}_{\mathbf{l}+\frac{1}{2}, \mathbf{m}+\frac{1}{2}, \mathbf{n}+\frac{1}{2}}(Z) & (l-m)(l-n+1) \mathbf{G}_{\mathbf{l}+\frac{1}{2}, \mathbf{m}+\frac{1}{2}, \mathbf{n}-\frac{1}{2}}(Z) \\
-(l+m+1)(l+n+2) \mathbf{G}_{\mathbf{l}+\frac{1}{2}, \mathbf{m}-\frac{1}{2}, \mathbf{n}+\frac{1}{2}}(Z) & (l+m+1)(l-n+1) \mathbf{G}_{\mathbf{l}+\frac{1}{2}, \mathbf{m}-\frac{1}{2}, \mathbf{n}-\frac{1}{2}}(Z)
\end{array}\right) \\
& +\frac{2 l(2 l+k+2)}{(2 l+1)^{2}}\left(\begin{array}{cc}
(l-m) \mathbf{H}_{\mathbf{k}, \mathbf{l}-\frac{1}{2}, \mathbf{m}+\frac{1}{2}, \mathbf{n}+\frac{1}{2}}(Z) & (l-m) \mathbf{H}_{\mathbf{k}, \mathbf{l}-\frac{1}{2}, \mathbf{m}+\frac{1}{2}, \mathbf{n}-\frac{1}{2}}(Z) \\
(l+m+1) \mathbf{H}_{\mathbf{k}, \mathbf{l}-\frac{1}{2}, \mathbf{m}-\frac{1}{2}, \mathbf{n}+\frac{1}{2}}(Z) & (l+m+1) \mathbf{H}_{\mathbf{k}, \mathbf{l}-\frac{1}{2}, \mathbf{m}-\frac{1}{2}, \mathbf{n}-\frac{1}{2}}(Z)
\end{array}\right) \\
& +\frac{k(2 l+4)}{(2 l+3)^{2}}\left(\begin{array}{cc}
(l+n+2) \mathbf{H}_{\mathbf{k}-\mathbf{1}, \mathbf{1}+\frac{1}{2}, \mathbf{m}+\frac{1}{2}, \mathbf{n}+\frac{1}{2}}(Z) & -(l-n+1) \mathbf{H}_{\mathbf{k}-\mathbf{1}, \mathbf{1}+\frac{1}{2}, \mathbf{m}+\frac{1}{2}, \mathbf{n}-\frac{1}{2}}(Z) \\
-(l+n+2) \mathbf{H}_{\mathbf{k}-\mathbf{1}, \mathbf{1}+\frac{1}{2}, \mathbf{m}-\frac{1}{2}, \mathbf{n}+\frac{1}{2}}(Z) & (l-n+1) \mathbf{H}_{\mathbf{k}-\mathbf{1}, \mathbf{1}+\frac{1}{2}, \mathbf{m}-\frac{1}{2}, \mathbf{n}-\frac{1}{2}}(Z)
\end{array}\right) ; \\
& \left(\begin{array}{cc}
\partial_{11}\left(N(Z)^{-2} \cdot Z^{+}\right) & \partial_{21}\left(N(Z)^{-2} \cdot Z^{+}\right) \\
\partial_{12}\left(N(Z)^{-2} \cdot Z^{+}\right) & \partial_{22}\left(N(Z)^{-2} \cdot Z^{+}\right)
\end{array}\right)=\frac{3}{2}\left(\begin{array}{cc}
-\mathbf{H}_{-\mathbf{2}, \mathbf{0}, \mathbf{0}, \mathbf{0}}(Z) & \mathbf{H}_{-\mathbf{2}, \mathbf{0}, \mathbf{0},-\mathbf{1}}(Z) \\
\mathbf{H}_{-\mathbf{2}, \mathbf{0},-\mathbf{1}, \mathbf{0}}(Z) & -\mathbf{H}_{-\mathbf{2 , 0 , - 1 , - 1}}(Z)
\end{array}\right) ; \\
& \left(\begin{array}{ll}
\partial_{11}\left(N(Z)^{k} \cdot \mathbf{F}_{1, \mathbf{m}, \mathbf{n}}^{\prime}(Z)\right) & \partial_{21}\left(N(Z)^{k} \cdot \mathbf{F}_{1, \mathbf{m}, \mathbf{n}}^{\prime}(Z)\right) \\
\partial_{12}\left(N(Z)^{k} \cdot \mathbf{F}_{\mathbf{1}, \mathbf{m}, \mathbf{n}}^{\prime}(Z)\right) & \partial_{22}\left(N(Z)^{k} \cdot \mathbf{F}_{\mathbf{1}, \mathbf{m}, \mathbf{n}}^{\prime}(Z)\right)
\end{array}\right) \\
& =\frac{k}{2 l} N(Z)^{k-1}\left(\begin{array}{cc}
(l+m+1) \mathbf{F}_{1-\frac{1}{2}, \mathbf{m}-\frac{1}{2}, \mathbf{n}-\frac{1}{2}}^{\prime}(Z) & -(l-m) \mathbf{F}_{1-\frac{1}{2}, \mathbf{m}+\frac{1}{2}, \mathbf{n}-\frac{1}{2}}^{\prime}(Z) \\
-(l+m+1) \mathbf{F}_{\mathbf{1}-\frac{1}{2}, \mathbf{m}-\frac{1}{2}, \mathbf{n}+\frac{1}{2}}^{\prime}(Z) & (l-m) \mathbf{F}_{\mathbf{1}-\frac{1}{2}, \mathbf{m}+\frac{1}{2}, \mathbf{n}+\frac{1}{2}}^{\prime}(Z)
\end{array}\right) \\
& -\frac{2 l-k+1}{2 l+2} N(Z)^{k}\left(\begin{array}{cc}
(l-n) \mathbf{F}_{\mathbf{1}+\frac{1}{2}, \mathbf{m}-\frac{1}{2}, \mathbf{n}-\frac{1}{2}}^{\prime}(Z) & (l-n) \mathbf{F}_{\mathbf{1}+\frac{1}{2}, \mathbf{m}+\frac{1}{2}, \mathbf{n}-\frac{1}{2}}^{\prime}(Z) \\
(l+n+1) \mathbf{F}_{\mathbf{1}+\frac{1}{2}, \mathbf{m}-\frac{1}{2}, \mathbf{n}+\frac{1}{2}}^{\prime}(Z) & (l+n+1) \mathbf{F}_{\mathbf{1}+\frac{1}{2}, \mathbf{m}+\frac{1}{2}, \mathbf{n}+\frac{1}{2}}^{\prime}(Z)
\end{array}\right) \\
& +\frac{1}{2 l+1}\left(\begin{array}{cc}
-(l+m+1)(l-n) \mathbf{H}_{\mathbf{k}-\mathbf{1}, \mathbf{1}-\frac{1}{2}, \mathbf{m}-\frac{1}{2}, \mathbf{n}-\frac{1}{2}}^{\prime}(Z) & (l-m)(l-n) \mathbf{H}_{\mathbf{k}-\mathbf{1}, \mathbf{l}-\frac{1}{2}, \mathbf{m}+\frac{1}{2}, \mathbf{n}-\frac{1}{2}}^{\prime}(Z) \\
-(l+m+1)(l+n+1) \mathbf{H}_{\mathbf{k}-\mathbf{1}, \mathbf{l}-\frac{1}{2}, \mathbf{m}-\frac{1}{2}, \mathbf{n}+\frac{1}{2}}^{\prime}(Z) & (l-m)(l+n+1) \mathbf{H}_{\mathbf{k}-\mathbf{1}, \mathbf{l}-\frac{1}{2}, \mathbf{m}+\frac{1}{2}, \mathbf{n}+\frac{1}{2}}^{\prime}(Z)
\end{array}\right) ;
\end{aligned}
$$




$$
\begin{aligned}
& \left(\begin{array}{ll}
\partial_{11}\left(N(Z)^{k} \cdot \mathbf{G}_{1, \mathbf{m}, \mathbf{n}}^{\prime}(Z)\right) & \partial_{21}\left(N(Z)^{k} \cdot \mathbf{G}_{1, \mathbf{m}, \mathbf{n}}^{\prime}(Z)\right) \\
\partial_{12}\left(N(Z)^{k} \cdot \mathbf{G}_{\mathbf{1}, \mathbf{m}, \mathbf{n}}^{\prime}(Z)\right) & \partial_{22}\left(N(Z)^{k} \cdot \mathbf{G}_{\mathbf{1}, \mathbf{m}, \mathbf{n}}^{\prime}(Z)\right)
\end{array}\right) \\
& =\frac{k}{2 l} N(Z)^{k-1}\left(\begin{array}{cc}
(l+m) \mathbf{G}_{\mathbf{1}-\frac{1}{2}, \mathbf{m}-\frac{1}{2}, \mathbf{n}-\frac{1}{2}}^{\prime}(Z) & -(l-m-1) \mathbf{G}_{1-\frac{1}{2}, \mathbf{m}+\frac{1}{2}, \mathbf{n}-\frac{1}{2}}^{\prime}(Z) \\
-(l+m) \mathbf{G}_{\mathbf{1}-\frac{1}{2}, \mathbf{m}-\frac{1}{2}, \mathbf{n}+\frac{1}{2}}^{\prime}(Z) & (l-m-1) \mathbf{G}_{\mathbf{1}-\frac{1}{2}, \mathbf{m}+\frac{1}{2}, \mathbf{n}+\frac{1}{2}}^{\prime}(Z)
\end{array}\right) \\
& -\frac{2 l-k+1}{2 l+2} N(Z)^{k}\left(\begin{array}{cc}
(l-n+1) \mathbf{G}_{1+\frac{1}{2}, \mathbf{m}-\frac{1}{2}, \mathbf{n}-\frac{1}{2}}^{\prime}(Z) & (l-n+1) \mathbf{G}_{\mathbf{1}+\frac{1}{2}, \mathbf{m}+\frac{1}{2}, \mathbf{n}-\frac{1}{2}}^{\prime}(Z) \\
(l+n+2) \mathbf{G}_{\mathbf{1}+\frac{1}{2}, \mathbf{m}-\frac{1}{2}, \mathbf{n}+\frac{1}{2}}^{\prime}(Z) & (l+n+2) \mathbf{G}_{\mathbf{1}+\frac{1}{2}, \mathbf{m}+\frac{1}{2}, \mathbf{n}+\frac{1}{2}}^{\prime}(Z)
\end{array}\right) \\
& +\frac{1}{2 l+1}\left(\begin{array}{cc}
-\mathbf{H}_{\mathbf{k}-\mathbf{1}, \mathbf{l}-\frac{1}{2}, \mathbf{m}-\frac{1}{2}, \mathbf{n}-\frac{1}{2}}^{\prime}(Z) & -\mathbf{H}_{\mathbf{k}-\mathbf{1}, \mathbf{l}-\frac{1}{2}, \mathbf{m}+\frac{1}{2}, \mathbf{n}-\frac{1}{2}}^{\prime}(Z) \\
\mathbf{H}_{\mathbf{k}-\mathbf{1}, \mathbf{l}-\frac{1}{2}, \mathbf{m}-\frac{1}{2}, \mathbf{n}+\frac{1}{2}}^{\prime}(Z) & \mathbf{H}_{\mathbf{k}-\mathbf{1}, \mathbf{l}-\frac{1}{2}, \mathbf{m}+\frac{1}{2}, \mathbf{n}+\frac{1}{2}}^{\prime}(Z)
\end{array}\right)
\end{aligned}
$$

$$
\begin{aligned}
& \left(\begin{array}{ll}
\partial_{11} \mathbf{H}_{\mathbf{k}, \mathbf{l}, \mathbf{m}, \mathbf{n}}^{\prime}(Z) & \partial_{21} \mathbf{H}_{\mathbf{k}, \mathbf{l}, \mathbf{m}, \mathbf{n}}^{\prime}(Z) \\
\partial_{12} \mathbf{H}_{\mathbf{k}, \mathbf{l}, \mathbf{m}, \mathbf{n}}^{\prime}(Z) & \partial_{22} \mathbf{H}_{\mathbf{k}, \mathbf{l}, \mathbf{m}, \mathbf{n}}^{\prime}(Z)
\end{array}\right) \\
& =\frac{(k+1)(2 l-k+1) N(Z)^{k}}{(l+1)(2 l+1)(2 l+3)}\left(\begin{array}{cc}
-\mathbf{F}_{1+\frac{1}{2}, \mathbf{m}-\frac{1}{2}, \mathbf{n}-\frac{1}{2}}^{\prime}(Z) & -\mathbf{F}_{1+\frac{1}{2}, \mathbf{m}+\frac{1}{2}, \mathbf{n}-\frac{1}{2}}^{\prime}(Z) \\
\mathbf{F}_{\mathbf{l}+\frac{1}{2}, \mathbf{m}-\frac{1}{2}, \mathbf{n}+\frac{1}{2}}^{\prime}(Z) & \mathbf{F}_{\mathbf{1}+\frac{1}{2}, \mathbf{m}+\frac{1}{2}, \mathbf{n}+\frac{1}{2}}^{\prime}(Z)
\end{array}\right) \\
& +\frac{(k+1)(2 l-k+1) N(Z)^{k}}{(l+1)(2 l+1)(2 l+3)} \\
& \times\left(\begin{array}{ll}
-(l+m+1)(l-n+1) \mathbf{G}_{1+\frac{1}{2}, \mathbf{m}-\frac{1}{2}, \mathbf{n}-\frac{1}{2}}^{\prime}(Z) & (l-m)(l-n+1) \mathbf{G}_{1+\frac{1}{2}, \mathbf{m}+\frac{1}{2}, \mathbf{n}-\frac{1}{2}}^{\prime}(Z) \\
-(l+m+1)(l+n+2) \mathbf{G}_{\mathbf{1}+\frac{1}{2}, \mathbf{m}-\frac{1}{2}, \mathbf{n}+\frac{1}{2}}^{\prime}(Z) & (l-m)(l+n+2) \mathbf{G}_{\mathbf{1 + \frac { 1 } { 2 }}, \mathbf{m}+\frac{1}{2}, \mathbf{n}+\frac{1}{2}}^{\prime}(Z)
\end{array}\right) \\
& +\frac{2 l(k+1)}{(2 l+1)^{2}}\left(\begin{array}{cc}
(l+m+1) \mathbf{H}_{\mathbf{k}-\mathbf{1}, \mathbf{l}-\frac{1}{2}, \mathbf{m}-\frac{1}{2}, \mathbf{n}-\frac{1}{2}}^{\prime}(Z) & -(l-m) \mathbf{H}_{\mathbf{k}-\mathbf{1}, \mathbf{1}-\frac{1}{2}, \mathbf{m}+\frac{1}{2}, \mathbf{n}-\frac{1}{2}}^{\prime}(Z) \\
-(l+m+1) \mathbf{H}_{\mathbf{k}-\mathbf{1}, \mathbf{l}-\frac{1}{2}, \mathbf{m}-\frac{1}{2}, \mathbf{n}+\frac{1}{2}}^{\prime}(Z) & (l-m) \mathbf{H}_{\mathbf{k}-\mathbf{1}, \mathbf{1}-\frac{1}{2}, \mathbf{m}+\frac{1}{2}, \mathbf{n}+\frac{1}{2}}^{\prime}(Z)
\end{array}\right) \\
& -\frac{(2 l+4)(2 l-k+1)}{(2 l+3)^{2}}\left(\begin{array}{cc}
(l-n+1) \mathbf{H}_{\mathbf{k}, \mathbf{l}+\frac{1}{2}, \mathbf{m}+\frac{1}{2}, \mathbf{n}+\frac{1}{2}}^{\prime}(Z) & (l-n+1) \mathbf{H}_{\mathbf{k}, \mathbf{l}+\frac{1}{2}, \mathbf{m}+\frac{1}{2}, \mathbf{n}-\frac{1}{2}}^{\prime}(Z) \\
(l+n+2) \mathbf{H}_{\mathbf{k}, \mathbf{l}+\frac{1}{2}, \mathbf{m}-\frac{1}{2}, \mathbf{n}+\frac{1}{2}}^{\prime}(Z) & (l+n+2) \mathbf{H}_{\mathbf{k}, \mathbf{l}+\frac{1}{2}, \mathbf{m}+\frac{1}{2}, \mathbf{n}+\frac{1}{2}}^{\prime}(Z)
\end{array}\right) .
\end{aligned}
$$

Let $\left(\begin{array}{ll}0 & 1 \\ 1 & 0\end{array}\right) \in G L\left(2, \mathbb{H}_{\mathbb{C}}\right)$, then

$$
\begin{aligned}
\rho_{2}\left(\begin{array}{ll}
0 & 1 \\
1 & 0
\end{array}\right)\left(N(Z)^{k} \cdot \mathbf{F}_{\mathbf{l}, \mathbf{m}, \mathbf{n}}(Z)\right) & =N(Z)^{-k-2} \cdot \mathbf{F}_{\mathbf{l}, \mathbf{m}, \mathbf{n}}^{\prime}(Z), \\
\rho_{2}\left(\begin{array}{ll}
0 & 1 \\
1 & 0
\end{array}\right)\left(N(Z)^{k} \cdot \mathbf{G}_{\mathbf{l}, \mathbf{m}, \mathbf{n}}(Z)\right) & =N(Z)^{-k-2} \cdot \mathbf{G}_{\mathbf{l}, \mathbf{m}, \mathbf{n}}^{\prime}(Z), \\
\rho_{2}\left(\begin{array}{ll}
0 & 1 \\
1 & 0
\end{array}\right)\left(\mathbf{H}_{\mathbf{k}, \mathbf{l}, \mathbf{m}, \mathbf{n}}(Z)\right) & =\mathbf{H}_{-\mathbf{k}-\mathbf{2}, \mathbf{l}, \mathbf{m}, \mathbf{n}}^{\prime}(Z), \\
\rho_{2}\left(\begin{array}{ll}
0 & 1 \\
1 & 0
\end{array}\right)\left(N(Z)^{-2} \cdot Z^{+}\right) & =-N(Z)^{-2} \cdot Z^{+} .
\end{aligned}
$$

Now we can compute the action of $\left(\begin{array}{cc}0 & 0 \\ C & 0\end{array}\right) \in \mathfrak{g l}\left(2, \mathbb{H}_{\mathbb{C}}\right), C \in \mathbb{H}_{\mathbb{C}}$, on these generators:

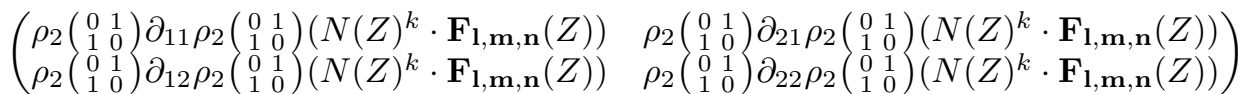

$$
\begin{aligned}
& =-\frac{k+2}{2 l} N(Z)^{k+1}\left(\begin{array}{cc}
(l+m+1) \mathbf{F}_{\mathbf{1}-\frac{1}{2}, \mathbf{m}-\frac{1}{2}, \mathbf{n}-\frac{1}{2}}(Z) & -(l-m) \mathbf{F}_{\mathbf{1}-\frac{1}{2}, \mathbf{m}+\frac{1}{2}, \mathbf{n}-\frac{1}{2}}(Z) \\
-(l+m+1) \mathbf{F}_{\mathbf{l}-\frac{1}{2}, \mathbf{m}-\frac{1}{2}, \mathbf{n}+\frac{1}{2}}(Z) & (l-m) \mathbf{F}_{\mathbf{1}-\frac{1}{2}, \mathbf{m}+\frac{1}{2}, \mathbf{n}+\frac{1}{2}}(Z)
\end{array}\right) \\
& -\frac{2 l+k+3}{2 l+2} N(Z)^{k}\left(\begin{array}{cc}
(l-n) \mathbf{F}_{\mathbf{l}+\frac{1}{2}, \mathbf{m}-\frac{1}{2}, \mathbf{n}-\frac{1}{2}}(Z) & (l-n) \mathbf{F}_{\mathbf{l}+\frac{1}{2}, \mathbf{m}+\frac{1}{2}, \mathbf{n}-\frac{1}{2}}(Z) \\
(l+n+1) \mathbf{F}_{\mathbf{1}+\frac{1}{2}, \mathbf{m}-\frac{1}{2}, \mathbf{n}+\frac{1}{2}}(Z) & (l+n+1) \mathbf{F}_{\mathbf{1}+\frac{1}{2}, \mathbf{m}+\frac{1}{2}, \mathbf{n}+\frac{1}{2}}(Z)
\end{array}\right) \\
& +\frac{1}{2 l+1}\left(\begin{array}{cc}
-(l+m+1)(l-n) \mathbf{H}_{\mathbf{k}+\mathbf{1}, \mathbf{l}-\frac{1}{2}, \mathbf{m}-\frac{1}{2}, \mathbf{n}-\frac{1}{2}}(Z) & (l-m)(l-n) \mathbf{H}_{\mathbf{k}+\mathbf{1}, \mathbf{1}-\frac{1}{2}, \mathbf{m}+\frac{1}{2}, \mathbf{n}-\frac{1}{2}}(Z) \\
-(l+m+1)(l+n+1) \mathbf{H}_{\mathbf{k}+\mathbf{1}, \mathbf{1}-\frac{1}{2}, \mathbf{m}-\frac{1}{2}, \mathbf{n}+\frac{1}{2}}(Z) & (l-m)(l+n+1) \mathbf{H}_{\mathbf{k}+\mathbf{1}, \mathbf{1}-\frac{1}{2}, \mathbf{m}+\frac{1}{2}, \mathbf{n}+\frac{1}{2}}(Z)
\end{array}\right) ;
\end{aligned}
$$




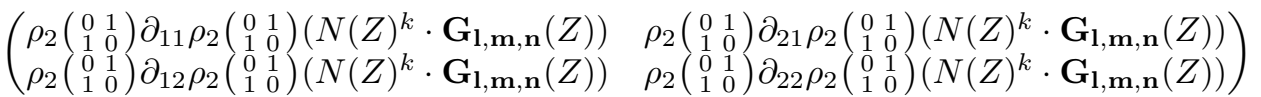

$$
\begin{aligned}
& =-\frac{k+2}{2 l} N(Z)^{k+1}\left(\begin{array}{cc}
(l+m) \mathbf{G}_{\mathbf{l}-\frac{1}{2}, \mathbf{m}-\frac{1}{2}, \mathbf{n}-\frac{1}{2}}(Z) & -(l-m-1) \mathbf{G}_{\mathbf{l}-\frac{1}{2}, \mathbf{m}+\frac{1}{2}, \mathbf{n}-\frac{1}{2}}(Z) \\
-(l+m) \mathbf{G}_{\mathbf{l}-\frac{1}{2}, \mathbf{m}-\frac{1}{2}, \mathbf{n}+\frac{1}{2}}(Z) & (l-m-1) \mathbf{G}_{\mathbf{l}-\frac{1}{2}, \mathbf{m}+\frac{1}{2}, \mathbf{n}+\frac{1}{2}}(Z)
\end{array}\right) \\
& -\frac{2 l+k+3}{2 l+2} N(Z)^{k}\left(\begin{array}{cc}
(l-n+1) \mathbf{G}_{\mathbf{l}+\frac{1}{2}, \mathbf{m}-\frac{1}{2}, \mathbf{n}-\frac{1}{2}}(Z) & (l-n+1) \mathbf{G}_{\mathbf{l}+\frac{1}{2}, \mathbf{m}+\frac{1}{2}, \mathbf{n}-\frac{1}{2}}(Z) \\
(l+n+2) \mathbf{G}_{\mathbf{l} \frac{1}{2}, \mathbf{m}-\frac{1}{2}, \mathbf{n}+\frac{1}{2}}(Z) & (l+n+2) \mathbf{G}_{\mathbf{l} \frac{1}{2}, \mathbf{m}+\frac{1}{2}, \mathbf{n}+\frac{1}{2}}(Z)
\end{array}\right) \\
& +\frac{1}{2 l+1}\left(\begin{array}{cc}
-\mathbf{H}_{\mathbf{k}+\mathbf{1}, \mathbf{l}-\frac{1}{2}, \mathbf{m}-\frac{1}{2}, \mathbf{n}-\frac{1}{2}}(Z) & -\mathbf{H}_{\mathbf{k}+\mathbf{1}, \mathbf{l}-\frac{1}{2}, \mathbf{m}+\frac{1}{2}, \mathbf{n}-\frac{1}{2}}(Z) \\
\mathbf{H}_{\mathbf{k}+\mathbf{1}, \mathbf{l}-\frac{1}{2}, \mathbf{m}-\frac{1}{2}, \mathbf{n}+\frac{1}{2}}(Z) & \mathbf{H}_{\mathbf{k}+\mathbf{1}, \mathbf{1}-\frac{1}{2}, \mathbf{m}+\frac{1}{2}, \mathbf{n}+\frac{1}{2}}(Z)
\end{array}\right) ;
\end{aligned}
$$

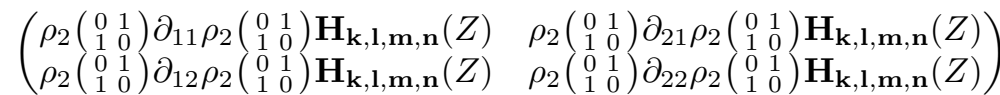

$$
\begin{aligned}
& =-\frac{(k+1)(2 l+k+3) N(Z)^{k}}{(l+1)(2 l+1)(2 l+3)}\left(\begin{array}{cc}
-\mathbf{F}_{\mathbf{1}+\frac{1}{2}, \mathbf{m}-\frac{1}{2}, \mathbf{n}-\frac{1}{2}}(Z) & -\mathbf{F}_{\mathbf{l}+\frac{1}{2}, \mathbf{m}+\frac{1}{2}, \mathbf{n}-\frac{1}{2}}(Z) \\
\mathbf{F}_{\mathbf{l}+\frac{1}{2}, \mathbf{m}-\frac{1}{2}, \mathbf{n}+\frac{1}{2}}(Z) & \mathbf{F}_{\mathbf{l}+\frac{1}{2}, \mathbf{m}+\frac{1}{2}, \mathbf{n}+\frac{1}{2}}(Z)
\end{array}\right) \\
& +\frac{(k+1)(2 l+k+3) N(Z)^{k}}{(l+1)(2 l+1)(2 l+3)} \\
& \times\left(\begin{array}{ll}
(l+m+1)(l-n+1) \mathbf{G}_{\mathbf{1}+\frac{1}{2}, \mathbf{m}-\frac{1}{2}, \mathbf{n}-\frac{1}{2}}(Z) & -(l-m)(l-n+1) \mathbf{G}_{\mathbf{1 + \frac { 1 } { 2 }}, \mathbf{m}+\frac{1}{2}, \mathbf{n}-\frac{1}{2}}(Z) \\
(l+m+1)(l+n+2) \mathbf{G}_{\mathbf{1} \frac{1}{2}, \mathbf{m}-\frac{1}{2}, \mathbf{n}+\frac{1}{2}}(Z) & -(l-m)(l+n+2) \mathbf{G}_{\mathbf{1}+\frac{1}{2}, \mathbf{m}+\frac{1}{2}, \mathbf{n}+\frac{1}{2}}(Z)
\end{array}\right) \\
& -\frac{2 l(k+1)}{(2 l+1)^{2}}\left(\begin{array}{cc}
(l+m+1) \mathbf{H}_{\mathbf{k}+\mathbf{1}, \mathbf{l}-\frac{1}{2}, \mathbf{m}-\frac{1}{2}, \mathbf{n}-\frac{1}{2}}(Z) & -(l-m) \mathbf{H}_{\mathbf{k}+\mathbf{1}, \mathbf{l}-\frac{1}{2}, \mathbf{m}+\frac{1}{2}, \mathbf{n}-\frac{1}{2}}(Z) \\
-(l+m+1) \mathbf{H}_{\mathbf{k}+\mathbf{1}, \mathbf{l}-\frac{1}{2}, \mathbf{m}-\frac{1}{2}, \mathbf{n}+\frac{1}{2}}(Z) & (l-m) \mathbf{H}_{\mathbf{k}+\mathbf{1}, \mathbf{l}-\frac{1}{2}, \mathbf{m}+\frac{1}{2}, \mathbf{n}+\frac{1}{2}}(Z)
\end{array}\right) \\
& -\frac{(2 l+4)(2 l+k+3)}{(2 l+3)^{2}}\left(\begin{array}{cc}
(l-n+1) \mathbf{H}_{\mathbf{k}, \mathbf{l}+\frac{1}{2}, \mathbf{m}+\frac{1}{2}, \mathbf{n}+\frac{1}{2}}(Z) & (l-n+1) \mathbf{H}_{\mathbf{k}, \mathbf{l}+\frac{1}{2}, \mathbf{m}+\frac{1}{2}, \mathbf{n}-\frac{1}{2}}(Z) \\
(l+n+2) \mathbf{H}_{\mathbf{k}, \mathbf{l}+\frac{1}{2}, \mathbf{m}-\frac{1}{2}, \mathbf{n}+\frac{1}{2}}(Z) & (l+n+2) \mathbf{H}_{\mathbf{k}, \mathbf{l}+\frac{1}{2}, \mathbf{m}+\frac{1}{2}, \mathbf{n}+\frac{1}{2}}(Z)
\end{array}\right) ;
\end{aligned}
$$

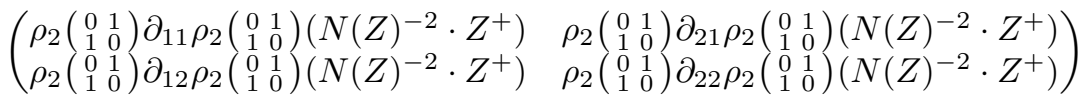

$$
\begin{aligned}
& =\frac{3}{2}\left(\begin{array}{cc}
\mathbf{H}_{\mathbf{0}, \mathbf{0}, \mathbf{0}, \mathbf{0}}^{\prime}(Z) & -\mathbf{H}_{\mathbf{0}, \mathbf{0}, \mathbf{0},-\mathbf{1}}^{\prime}(Z) \\
-\mathbf{H}_{\mathbf{0}, \mathbf{0},-\mathbf{1}, \mathbf{0}}^{\prime}(Z) & \mathbf{H}_{\mathbf{0}, \mathbf{0},-\mathbf{1},-\mathbf{1}}(Z)
\end{array}\right)=-\frac{3}{2}\left(\begin{array}{cc}
\mathbf{H}_{-\mathbf{1}, \mathbf{0},-\mathbf{1},-\mathbf{1}}(Z) & \mathbf{H}_{-\mathbf{1 , 0 , 0},-\mathbf{1}}(Z) \\
\mathbf{H}_{-\mathbf{1 , 0},-\mathbf{1}, \mathbf{0}}(Z) & \mathbf{H}_{-\mathbf{1 , 0 , 0}, \mathbf{0}}(Z)
\end{array}\right),
\end{aligned}
$$

From (52)-(54) we see that any $\rho_{2}$-invariant subspace of the kernel of $\operatorname{Tr} \circ \partial^{+}: \mathcal{W} \rightarrow \amalg$ contains at least one function $\mathbf{H}_{\mathbf{k}, \mathbf{l}, \mathbf{m}, \mathbf{n}}(Z)$. We introduce a $U(2) \times U(2)$-invariant subspace of $\mathcal{W}$

$$
\mathbf{H}=\mathbb{C} \text {-span of }\left\{\mathbf{H}_{\mathbf{k}, \mathbf{l}, \mathbf{m}, \mathbf{n}}(Z) ; k=0, \pm 1, \pm 2, \ldots, l=0, \frac{1}{2}, 1, \frac{3}{2}, \ldots,-l-1 \leq m, n \leq l\right\}
$$

- note that $\mathbf{H}$ is not $\mathfrak{g l}\left(2, \mathbb{H}_{\mathbb{C}}\right)$-invariant - and a projection

$$
\varpi_{\mathbf{H}}: \operatorname{ker}\left(\operatorname{Tr} \circ \partial^{+}: \mathcal{W} \rightarrow \amalg I\right) \rightarrow \mathbf{H}
$$

that maps each $\mathbf{H}_{\mathbf{k}, \mathbf{l}, \mathbf{m}, \mathbf{n}}(Z)$ into itself and annihilates $Z^{+} \cdot N(Z)^{2}$, all $N(Z)^{k} \cdot \mathbf{F}_{\mathbf{l}, \mathbf{m}, \mathbf{n}}(Z)^{\text {'s }}$ and all $N(Z)^{k} \cdot \mathbf{G}_{\mathbf{l}, \mathbf{m}, \mathbf{n}}(Z)$ 's. Then

$$
\begin{aligned}
& \left(\begin{array}{ll}
\varpi_{\mathbf{H}} \circ \partial_{11} \mathbf{H}_{\mathbf{k}, \mathbf{l}, \mathbf{m}, \mathbf{n}}(Z) & \varpi_{\mathbf{H}} \circ \partial_{21} \mathbf{H}_{\mathbf{k}, \mathbf{l}, \mathbf{m}, \mathbf{n}}(Z) \\
\varpi_{\mathbf{H}} \circ \partial_{12} \mathbf{H}_{\mathbf{k}, \mathbf{l}, \mathbf{m}, \mathbf{n}}(Z) & \varpi_{\mathbf{H}} \circ \partial_{22} \mathbf{H}_{\mathbf{k}, \mathbf{l}, \mathbf{m}, \mathbf{n}}(Z)
\end{array}\right) \\
& =\frac{2 l(2 l+k+2)}{(2 l+1)^{2}}\left(\begin{array}{cc}
(l-m) \mathbf{H}_{\mathbf{k}, \mathbf{l}-\frac{1}{2}, \mathbf{m}+\frac{1}{2}, \mathbf{n}+\frac{1}{2}}(Z) & (l-m) \mathbf{H}_{\mathbf{k}, \mathbf{l}-\frac{1}{2}, \mathbf{m}+\frac{1}{2}, \mathbf{n}-\frac{1}{2}}(Z) \\
(l+m+1) \mathbf{H}_{\mathbf{k}, \mathbf{l}-\frac{1}{2}, \mathbf{m}-\frac{1}{2}, \mathbf{n}+\frac{1}{2}}(Z) & (l+m+1) \mathbf{H}_{\mathbf{k}, \mathbf{l}-\frac{1}{2}, \mathbf{m}-\frac{1}{2}, \mathbf{n}-\frac{1}{2}}(Z)
\end{array}\right) \\
& +\frac{k(2 l+4)}{(2 l+3)^{2}}\left(\begin{array}{cc}
(l+n+2) \mathbf{H}_{\mathbf{k}-\mathbf{1}, \mathbf{l}+\frac{1}{2}, \mathbf{m}+\frac{1}{2}, \mathbf{n}+\frac{1}{2}}(Z) & -(l-n+1) \mathbf{H}_{\mathbf{k}-\mathbf{1}, \mathbf{l}+\frac{1}{2}, \mathbf{m}+\frac{1}{2}, \mathbf{n}-\frac{1}{2}}(Z) \\
-(l+n+2) \mathbf{H}_{\mathbf{k}-\mathbf{1}, \mathbf{l}+\frac{1}{2}, \mathbf{m}-\frac{1}{2}, \mathbf{n}+\frac{1}{2}}(Z) & (l-n+1) \mathbf{H}_{\mathbf{k}-\mathbf{1}, \mathbf{l}+\frac{1}{2}, \mathbf{m}-\frac{1}{2}, \mathbf{n}-\frac{1}{2}}(Z)
\end{array}\right)
\end{aligned}
$$




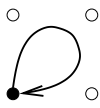

(a) Actions of $\rho_{2}\left(\begin{array}{cc}A & 0 \\ 0 & 0\end{array}\right)$ and $\rho_{2}\left(\begin{array}{ll}0 & 0 \\ 0 & D\end{array}\right)$

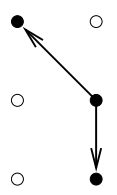

(b) Action of $\varpi_{\mathbf{H}} \circ \rho_{2}\left(\begin{array}{ll}0 & B \\ 0 & 0\end{array}\right)$

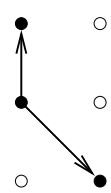

(c) Action of $\varpi_{\mathbf{H}} \circ \rho_{2}\left(\begin{array}{cc}0 & 0 \\ C & 0\end{array}\right)$

Figure 6

and

$$
\begin{aligned}
& \left(\begin{array}{ll}
\varpi_{\mathbf{H}} \circ \rho_{2}\left(\begin{array}{ll}
0 & 1 \\
1 & 0
\end{array}\right) \partial_{11} \rho_{2}\left(\begin{array}{ll}
0 & 1 \\
1 & 0
\end{array}\right) \mathbf{H}_{\mathbf{k}, \mathbf{l}, \mathbf{m}, \mathbf{n}}(Z) & \varpi_{\mathbf{H}} \circ \rho_{2}\left(\begin{array}{ll}
0 & 1 \\
1 & 0
\end{array}\right) \partial_{21} \rho_{2}\left(\begin{array}{ll}
0 & 1 \\
1 & 0
\end{array}\right) \mathbf{H}_{\mathbf{k}, \mathbf{l}, \mathbf{m}, \mathbf{n}}(Z) \\
\varpi_{\mathbf{H}} \circ \rho_{2}\left(\begin{array}{ll}
0 & 1 \\
1 & 0
\end{array}\right) \partial_{12} \rho_{2}\left(\begin{array}{ll}
0 & 1 \\
1 & 0
\end{array}\right) \mathbf{H}_{\mathbf{k}, \mathbf{l}, \mathbf{m}, \mathbf{n}}(Z) & \varpi_{\mathbf{H}} \circ \rho_{2}\left(\begin{array}{ll}
0 & 1 \\
1 & 0
\end{array}\right) \partial_{22} \rho_{2}\left(\begin{array}{ll}
0 & 1 \\
1 & 0
\end{array}\right) \mathbf{H}_{\mathbf{k}, \mathbf{l}, \mathbf{m}, \mathbf{n}}(Z)
\end{array}\right) \\
& =-\frac{2 l(k+1)}{(2 l+1)^{2}}\left(\begin{array}{cc}
(l+m+1) \mathbf{H}_{\mathbf{k}+\mathbf{1}, \mathbf{l}-\frac{1}{2}, \mathbf{m}-\frac{1}{2}, \mathbf{n}-\frac{1}{2}}(Z) & -(l-m) \mathbf{H}_{\mathbf{k}+\mathbf{1}, \mathbf{l}-\frac{1}{2}, \mathbf{m}+\frac{1}{2}, \mathbf{n}-\frac{1}{2}}(Z) \\
-(l+m+1) \mathbf{H}_{\mathbf{k}+\mathbf{1}, \mathbf{l}-\frac{1}{2}, \mathbf{m}-\frac{1}{2}, \mathbf{n}+\frac{1}{2}}(Z) & (l-m) \mathbf{H}_{\mathbf{k}+\mathbf{1}, \mathbf{l}-\frac{1}{2}, \mathbf{m}+\frac{1}{2}, \mathbf{n}+\frac{1}{2}}(Z)
\end{array}\right) \\
& -\frac{(2 l+4)(2 l+k+3)}{(2 l+3)^{2}}\left(\begin{array}{ll}
(l-n+1) \mathbf{H}_{\mathbf{k}, \mathbf{l}+\frac{1}{2}, \mathbf{m}+\frac{1}{2}, \mathbf{n}+\frac{1}{2}}(Z) & (l-n+1) \mathbf{H}_{\mathbf{k}, \mathbf{l}+\frac{1}{2}, \mathbf{m}+\frac{1}{2}, \mathbf{n}-\frac{1}{2}}(Z) \\
(l+n+2) \mathbf{H}_{\mathbf{k}, \mathbf{l}+\frac{1}{2}, \mathbf{m}-\frac{1}{2}, \mathbf{n}+\frac{1}{2}}(Z) & (l+n+2) \mathbf{H}_{\mathbf{k}, \mathbf{l}+\frac{1}{2}, \mathbf{m}+\frac{1}{2}, \mathbf{n}+\frac{1}{2}}(Z)
\end{array}\right)
\end{aligned}
$$

The actions of $\left(\begin{array}{ll}A & 0 \\ 0 & 0\end{array}\right),\left(\begin{array}{ll}0 & B \\ 0 & 0\end{array}\right),\left(\begin{array}{ll}0 & 0 \\ C & 0\end{array}\right)$ and $\left(\begin{array}{ll}0 & 0 \\ 0 & D\end{array}\right)$ are illustrated in Figure 6 . In the diagram describing $\varpi_{\mathbf{H}} \circ \rho_{2}\left(\begin{array}{ll}0 & B \\ 0 & 0\end{array}\right)$ the vertical arrow disappears if $l=0$ or $2 l+k+2=0$ and the diagonal arrow disappears if $k=0$. Similarly, in the diagram describing $\varpi_{\mathbf{H}} \circ \rho_{2}\left(\begin{array}{ll}0 & 0 \\ C & 0\end{array}\right)$ the vertical arrow disappears if $2 l+k+3=0$ and the diagonal arrow disappears if $k=-1$ or $l=0$. Let

$$
\begin{aligned}
\mathbf{H}^{+} & =\mathbb{C} \text {-span of }\left\{\mathbf{H}_{\mathbf{k}, \mathbf{l}, \mathbf{m}, \mathbf{n}}(Z) ; k \geq 0\right\}, \\
\mathbf{H}^{-} & =\mathbb{C} \text {-span of }\left\{\mathbf{H}_{\mathbf{k}, \mathbf{l}, \mathbf{m}, \mathbf{n}}(Z) ; k \leq-(2 l+3)\right\}, \\
\mathbf{H}^{0} & =\mathbb{C} \text {-span of }\left\{\mathbf{H}_{\mathbf{k}, \mathbf{l}, \mathbf{m}, \mathbf{n}}(Z) ;-(2 l+2) \leq k \leq-1\right\}
\end{aligned}
$$

(see Figure 7 and compare with Figure 2 in [FL3]). It follows that if $\mathcal{U}$ is a $\rho_{2}$-invariant subspace of $\operatorname{ker}\left(\operatorname{Tr} \circ \partial^{+}: \mathcal{W} \rightarrow W\right)$, then $\varpi_{\mathbf{H}}(\mathcal{U})$ must be $\mathbf{H}^{-}, \mathbf{H}^{0}, \mathbf{H}^{+}$, a direct sum of two of these spaces or all of $\mathbf{H}$.

Now let

$$
\begin{aligned}
& \mathcal{Q}^{+}=\mathbb{C} \text {-span of }\left\{N(Z)^{k} \cdot \mathbf{F}_{\mathbf{l}, \mathbf{m}, \mathbf{n}}(Z), N(Z)^{k} \cdot \mathbf{G}_{\mathbf{l}, \mathbf{m}, \mathbf{n}}(Z), \mathbf{H}_{\mathbf{k}, \mathbf{l}, \mathbf{m}, \mathbf{n}}(Z) ; k \geq 0\right\}, \\
& \mathcal{Q}^{-}=\mathbb{C} \text {-span of }\left\{N(Z)^{k} \cdot \mathbf{F}_{\mathbf{l}, \mathbf{m}, \mathbf{n}}(Z), N(Z)^{k} \cdot \mathbf{G}_{\mathbf{l}, \mathbf{m}, \mathbf{n}}(Z), \mathbf{H}_{\mathbf{k}, \mathbf{l}, \mathbf{m}, \mathbf{n}}(Z) ; k \leq-(2 l+3)\right\}, \\
& \mathcal{Q}^{0}=\mathbb{C} \text {-span of }\left\{\begin{array}{c}
N(Z)^{k} \cdot \mathbf{F}_{\mathbf{l}, \mathbf{m}, \mathbf{n}}(Z), N(Z)^{k} \cdot \mathbf{G}_{\mathbf{l}, \mathbf{m}, \mathbf{n}}(Z) \text { with }-(2 l+1) \leq k \leq-2, \\
\mathbf{H}_{\mathbf{k}, \mathbf{l}, \mathbf{m}, \mathbf{n}}(Z) \text { with }-(2 l+2) \leq k \leq-1
\end{array}\right\} .
\end{aligned}
$$

It is easy to see that

$$
\varpi_{\mathbf{H}}\left(\mathcal{Q}^{+}\right)=\mathbf{H}^{+}, \quad \varpi_{\mathbf{H}}\left(\mathcal{Q}^{-}\right)=\mathbf{H}^{-}, \quad \varpi_{\mathbf{H}}\left(\mathcal{Q}^{0}\right)=\mathbf{H}^{0},
$$

$\mathcal{Q}^{+}, \mathcal{Q}^{-}, \mathcal{Q}^{0}$ are invariant under the $\rho_{2}$-action of $\mathfrak{g l}\left(2, \mathbb{H}_{\mathbb{C}}\right)$ and irreducible. Moreover, these are the only irreducible subrepresentations of $\left(\rho_{2}, \operatorname{ker}\left(\operatorname{Tr} \circ \partial^{+}\right)\right)$. Furthermore, the quotient

$$
\frac{\operatorname{ker}\left(\operatorname{Tr} \circ \partial^{+}: \mathcal{W} \rightarrow H\right)}{\mathcal{Q}^{-} \oplus \mathcal{Q}^{0} \oplus \mathcal{Q}^{+}}
$$




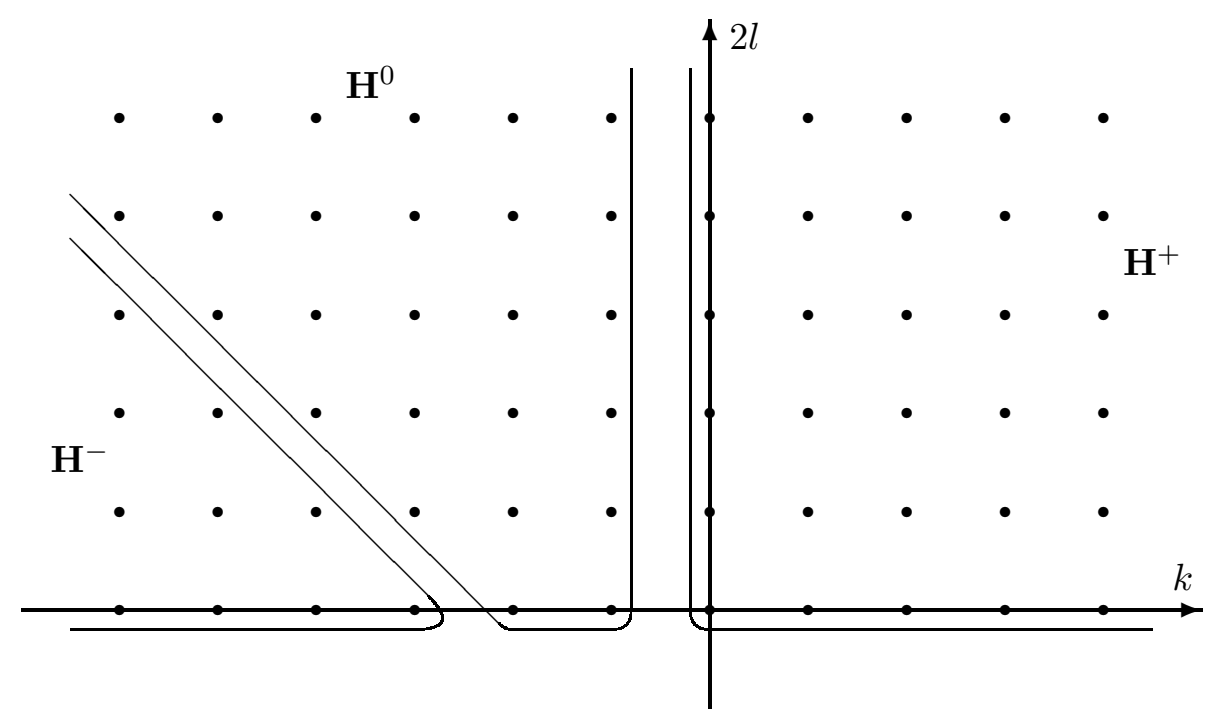

Figure 7: Decomposition of $\left(\varpi_{\mathbf{H}} \circ \rho_{2}, \mathbf{H}\right)$ into invariant components.

decomposes into five irreducible subrepresentations; they are the images of

$$
\begin{aligned}
& \mathbb{C} \text {-span of }\left\{N(Z)^{-1} \cdot \mathbf{F}_{\mathbf{l}, \mathbf{m}, \mathbf{n}}(Z) ; l \geq 1 / 2\right\}, \\
& \mathbb{C} \text {-span of }\{\left\{N(Z)^{-(2 l+2)} \cdot \mathbf{G}_{\mathbf{l}, \mathbf{m}, \mathbf{n}}(Z) ; l \geq 1 / 2\right\} \\
&=\mathbb{C} \text {-span of }\left\{N(Z)^{-1} \cdot \mathbf{F}_{1, \mathbf{m}, \mathbf{n}}^{\prime}(Z) ; l \geq 1 / 2\right\}, \\
& \mathbb{C} \text {-span of }\{\left\{N(Z)^{-1} \cdot \mathbf{G}_{\mathbf{l}, \mathbf{m}, \mathbf{n}}(Z) ; l \geq 1 / 2\right\}, \\
& \mathbb{C} \text {-span of }\{\left\{N(Z)^{-(2 l+2)} \cdot \mathbf{F}_{\mathbf{l}, \mathbf{m}, \mathbf{n}}(Z) ; l \geq 1 / 2\right\} \\
&=\mathbb{C} \text {-span of }\left\{N(Z)^{-1} \cdot \mathbf{G}_{\mathbf{l}, \mathbf{m}, \mathbf{n}}^{\prime}(Z) ; l \geq 1 / 2\right\}, \\
& \mathbb{C} \text {-span of }\left\{N(Z)^{-2} \cdot Z^{+}\right\} .
\end{aligned}
$$

These subrepresentations are isomorphic to

$$
\left(\rho_{2}^{\prime}, \mathcal{W}^{\prime} / \operatorname{ker} \tau_{a}^{+}\right), \quad\left(\rho_{2}^{\prime}, \mathcal{W}^{\prime} / \operatorname{ker} \tau_{a}^{-}\right), \quad\left(\rho_{2}^{\prime}, \mathcal{W}^{\prime} / \operatorname{ker} \tau_{s}^{+}\right), \quad\left(\rho_{2}^{\prime}, \mathcal{W}^{\prime} / \operatorname{ker} \tau_{s}^{-}\right)
$$

(which appeared in Corollary [51) and the trivial one-dimensional representation respectively. Combining this with Corollary 56, we obtain a description of all thirteen irreducible components of $\left(\rho_{2}, \mathcal{W}\right)$.

For future reference we make the following observation:

Lemma 59. The element $N(Z)^{-2} \cdot Z^{+} \in \mathcal{W}$ generates a subrepresentation $\mathcal{U}$ of $\mathcal{W}$ that has exactly two irreducible components: $\mathcal{Q}^{0}$ and the trivial one-dimensional representation. Moreover, the trivial one-dimensional representation does not appear as a subrepresentation of $\mathcal{U}$ it is isomorphic to the quotient $\mathcal{U} / \mathcal{Q}^{0}$.

Proof. The result follows from equations (54), (55).

\subsection{Decomposition of $\left(\rho_{2}^{\prime}, \mathcal{W}^{\prime}\right)$}

By Proposition 80 in [FL1, the representations $\left(\rho_{2}, \mathcal{W}\right)$ and $\left(\rho_{2}^{\prime}, \mathcal{W}^{\prime}\right)$ of $\mathfrak{g l}\left(2, \mathbb{H}_{\mathbb{C}}\right)$ are dual to each other. Thus the irreducible components of $\left(\rho_{2}^{\prime}, \mathcal{W}^{\prime}\right)$ are dual to those of $\left(\rho_{2}, \mathcal{W}\right)$, and, in 
particular, these two representations have the same number of irreducible components - thirteen. We would like to describe the irreducible components of $\left(\rho_{2}^{\prime}, \mathcal{W}^{\prime}\right)$ more explicitly.

The idea is to use the quaternionic chain complex (29) and deal separately with ker $\mathrm{Mx} \subset \mathcal{W}^{\prime}$ and $\mathcal{W}^{\prime} /$ ker Mx. Since ker Mx contains the image $\partial^{+}\left(W^{\prime}\right) \simeq W^{\prime} / \operatorname{ker} \partial^{+}=W^{\prime} / \mathcal{I}_{0}^{\prime}$, the following five irreducible components listed in Theorem 32 reappear in $\left(\rho_{2}^{\prime}, \mathcal{W}^{\prime}\right)$ :

$$
\left(\rho^{\prime}, \mathcal{B H}^{+} / \mathcal{I}_{0}^{\prime}\right), \quad\left(\rho^{\prime}, \mathcal{B H}^{-} / \mathcal{I}_{0}^{\prime}\right), \quad\left(\rho^{\prime}, H^{+} / \mathcal{B H}^{+}\right), \quad\left(\rho^{\prime}, H^{-} / \mathcal{B H}^{-}\right), \quad\left(\rho^{\prime}, \mathcal{J}^{\prime} /\left(\mathcal{B H}^{+}+\mathcal{B H}^{-}\right)\right) .
$$

The $\rho_{2}^{\prime}$-invariant subspace $\mathcal{M} \subset$ ker $\mathrm{Mx}$ contains all of the above components plus five more: four that appeared in Corollary 51 .

$$
\left(\rho_{2}^{\prime}, \mathcal{W}^{\prime} / \operatorname{ker} \tau_{a}^{+}\right), \quad\left(\rho_{2}^{\prime}, \mathcal{W}^{\prime} / \operatorname{ker} \tau_{a}^{-}\right), \quad\left(\rho_{2}^{\prime}, \mathcal{W}^{\prime} / \operatorname{ker} \tau_{s}^{+}\right), \quad\left(\rho_{2}^{\prime}, \mathcal{W}^{\prime} / \operatorname{ker} \tau_{s}^{-}\right)
$$

as well as the trivial one-dimensional representation spanned by $N(Z)^{-1} \cdot Z$. By Proposition 37 , these are the ten irreducible components of ker $\mathrm{Mx}$. It remains to describe the three irreducible components of $\mathcal{W}^{\prime} /$ ker $\mathrm{Mx}$ that are dual to $\left(\rho_{2}, \mathcal{Q}^{+}\right),\left(\rho_{2}, \mathcal{Q}^{-}\right)$and $\left(\rho_{2}, \mathcal{Q}^{0}\right)$.

Recall the functions $\tilde{F}_{l, m, n}(Z), \tilde{G}_{l, m, n}(Z), \tilde{H}_{l, m, n}(Z)$ from Proposition 42, We also introduce functions

$$
\begin{aligned}
& \tilde{I}_{k, l, m, n}(Z)=(2 l+k+2) N(Z)^{k} \cdot\left(\begin{array}{cc}
(l+m+1) t_{n \underline{m}}^{l}(Z) & -(l-m) t_{n \underline{m+1}}^{l}(Z) \\
-(l+m+1) t_{n+1}^{l} \underline{m}(Z) & (l-m) t_{n+1}^{l} \underline{m+1}(Z)
\end{array}\right) \\
& +k N(Z)^{k-1} \cdot\left(\begin{array}{cc}
(l-n+1) t_{n \underline{m}}^{l+1}(Z) & (l-n+1) t_{n}^{l+1}(Z+1) \\
(l+n+2) t_{n+1}^{l+1}(Z) & k= \pm 1, \pm 2, \pm 3, \ldots \\
l=-\frac{1}{2}, 0, \frac{1}{2}, 1, \frac{3}{2}, \ldots \\
-l-1 \leq m, n \leq l
\end{array}\right.
\end{aligned}
$$

(when $l=-\frac{1}{2}$, the function $\tilde{I}_{k,-\frac{1}{2},-\frac{1}{2},-\frac{1}{2}}(Z)$ reduces to $k N(Z)^{k-1} \cdot Z$ ) and

$$
\tilde{J}_{l, m, n}(Z)=N(Z)^{-1} \cdot\left(\begin{array}{cc}
(l-n) t_{n \underline{m}}^{l}(Z) & (l-n) t_{n+\underline{m+1}}^{l}(Z) \\
(l+n+1) t_{n+1 \underline{m}}^{l}(Z) & (l+n+1) t_{n+1}^{l \underline{m+1}}(Z)
\end{array}\right), \quad \begin{gathered}
l=\frac{1}{2}, 1, \frac{3}{2}, 2, \ldots \\
-l \leq m, n \leq l-1
\end{gathered} .
$$

Note that:

when $k=1$, the function $\tilde{I}_{1, l, m, n}(Z)$ is $\tilde{I}_{l+1, m, n}(Z)$ introduced after Lemma 44 ,

when $2 l+k+2=0$, the function $\tilde{I}_{k, l, m, n}(Z)$ is proportional to $\tilde{H}_{l+1,-n-1,-m-1}^{\prime}(Z)$,

when $2 l+k+1=0$, the function $\tilde{I}_{k, l, m, n}(Z)$ is proportional to $\tilde{I}_{l,-n-1,-m-1}^{\prime}(Z)$,

$$
\tilde{J}_{\frac{1}{2},-\frac{1}{2},-\frac{1}{2}}(Z)=N(Z)^{-1} \cdot Z \text {. }
$$

Recall that $K=U(2) \times U(2)$ sitting as the diagonal subgroup of $G L\left(2, \mathbb{H}_{\mathbb{C}}\right)$.

Proposition 60. The functions

$$
N(Z)^{k} \cdot \tilde{F}_{l, m, n}(Z), \quad N(Z)^{k} \cdot \tilde{G}_{l, m, n}(Z), \quad N(Z)^{k} \cdot \tilde{H}_{l, m, n}(Z), \quad \tilde{I}_{k, l, m, n}(Z), \quad \tilde{J}_{l, m, n}(Z)
$$

span $\mathcal{W}^{\prime}$ and generate the $K$-types of $\left(\rho_{2}^{\prime}, \mathcal{W}^{\prime}\right)$. More precisely, as representations of $S U(2) \times$ $S U(2)$,

$$
\begin{aligned}
& V_{l-\frac{1}{2}} \otimes V_{l+\frac{1}{2}}=\mathbb{C} \text {-span of }\left\{N(Z)^{k} \cdot \tilde{F}_{l, m, n}(Z) ; \begin{array}{l}
-l-1 \leq m \leq l \\
-l \leq n \leq l-1
\end{array}\right\}, \\
& V_{l+\frac{1}{2}} \otimes V_{l-\frac{1}{2}}=\mathbb{C} \text {-span of }\left\{N(Z)^{k} \cdot \tilde{G}_{l, m, n}(Z) ; \begin{array}{l}
-l \leq m \leq l-1 \\
-l-1 \leq n \leq l
\end{array}\right\}, \\
& V_{l+\frac{1}{2}} \otimes V_{l+\frac{1}{2}}=\mathbb{C} \text {-span of }\left\{N(Z)^{k} \cdot \tilde{H}_{l, m, n}(Z) ;-l-1 \leq m, n \leq l\right\}, \\
& V_{l+\frac{1}{2}} \otimes V_{l+\frac{1}{2}}=\mathbb{C} \text {-span of }\left\{\tilde{I}_{k, l, m, n}(Z) ;-l-1 \leq m, n \leq l\right\}, \\
& V_{l-\frac{1}{2}} \otimes V_{l-\frac{1}{2}}=\mathbb{C} \text {-span of }\left\{\tilde{J}_{l, m, n}(Z) ;-l \leq m, n \leq l-1\right\},
\end{aligned}
$$

for $k$ and $l$ fixed. 
Proof. Clearly, these functions are $K$-finite and linearly independent. One checks that these functions span all of $\mathcal{W}^{\prime}$ by checking, for each $d \in \mathbb{Z}$, that these functions generate all the $K$-types of

$$
\mathcal{W}^{\prime}(d)=\left\{F(Z) \in \mathcal{W}^{\prime} ; F(Z) \text { is homogeneous of degree } d\right\}
$$

Lemma 61. We have:

$$
\partial^{+}(W)=\mathbb{C} \text {-span of }\left\{\tilde{H}_{l, m, n}(Z) ; \begin{array}{l}
l=0, \frac{1}{2}, 1, \frac{3}{2}, \ldots \\
-l-1 \leq m, n \leq l
\end{array}\right\} \bigoplus \mathbb{C} \text {-span of }\left\{\begin{array}{l}
\tilde{I}_{k, l, m, n}(Z) ; \begin{array}{r}
k= \pm 1, \pm 2, \pm 3, \ldots \\
l=-\frac{1}{2}, 0, \frac{1}{2}, 1, \frac{3}{2}, \ldots \\
-l-1 \leq m, n \leq l
\end{array}
\end{array}\right\} \text {. }
$$

Proof. By Corollary 6 from [FL3],

$$
W=\mathbb{C} \text {-span of }\left\{N(Z)^{k} \cdot t_{n \underline{m}}^{l}(Z) ; \begin{array}{c}
k=0, \pm 1, \pm 2, \ldots \\
l=0, \frac{1}{2}, 1, \frac{3}{2}, \ldots \\
-l \leq m, n \leq l
\end{array}\right\} .
$$

Using Lemma 21 in [FL1 and identity (34), we obtain:

$$
\begin{aligned}
\partial^{+}\left(t_{0 \underline{0}}^{0}(Z)\right) & =0, \\
\partial^{+}\left(t_{n \underline{m}}^{l}(Z)\right) & =\tilde{H}_{l-\frac{1}{2}, m-\frac{1}{2}, n-\frac{1}{2}}(Z) \quad \text { if } l>0, \\
\partial^{+}\left(N(Z)^{k} \cdot t_{n \underline{m}}^{l}(Z)\right) & =\frac{1}{2 l+1} \tilde{I}_{k, l-\frac{1}{2}, m-\frac{1}{2}, n-\frac{1}{2}}(Z) \quad \text { if } k \neq 0 .
\end{aligned}
$$

Now let

$$
\begin{aligned}
& \mathcal{Q}^{++}=\mathbb{C} \text {-span of }\left\{N(Z)^{k} \cdot \tilde{F}_{l, m, n}(Z), N(Z)^{k} \cdot \tilde{G}_{l, m, n}(Z), N(Z)^{k} \cdot \tilde{H}_{l, m, n}(Z) ; k \geq 1\right\}, \\
& \mathcal{Q}^{\prime-}=\mathbb{C} \text {-span of }\left\{N(Z)^{k} \cdot \tilde{F}_{l, m, n}(Z), N(Z)^{k} \cdot \tilde{G}_{l, m, n}(Z), N(Z)^{k} \cdot \tilde{H}_{l, m, n}(Z) ; k \leq-(2 l+2)\right\}, \\
& \mathcal{Q}^{\prime 0}=\mathbb{C} \text {-span of }\left\{\begin{array}{c}
N(Z)^{k} \cdot \tilde{F}_{l, m, n}(Z), N(Z)^{k} \cdot \tilde{G}_{l, m, n}(Z) \text { with }-2 l \leq k \leq-1, \\
N(Z)^{k} \cdot \tilde{H}_{l, m, n}(Z) \text { with }-(2 l+1) \leq k \leq-1, \\
\tilde{J}_{l, m, n}(Z) \text { with } l=1, \frac{3}{2}, 2, \frac{5}{2}, \ldots
\end{array}\right\} ;
\end{aligned}
$$

we treat $\mathcal{Q}^{\prime+}, \mathcal{Q}^{\prime-}$ and $\mathcal{Q}^{\prime 0}$ as subspaces of the quotient space $\mathcal{W}^{\prime} /$ ker $\mathrm{Mx}$.

Theorem 62. The quotient representation $\left(\rho_{2}^{\prime}, \mathcal{W}^{\prime} / \operatorname{ker} \mathrm{Mx}\right)$ is the direct sum of three irreducible components $\left(\rho_{2}^{\prime}, \mathcal{Q}^{\prime+}\right),\left(\rho_{2}^{\prime}, \mathcal{Q}^{\prime-}\right)$ and $\left(\rho_{2}^{\prime}, \mathcal{Q}^{\prime 0}\right)$. These irreducible components are dual to $\left(\rho_{2}, \mathcal{Q}^{-}\right),\left(\rho_{2}, \mathcal{Q}^{+}\right)$and $\left(\rho_{2}, \mathcal{Q}^{0}\right)$ respectively via the invariant bilinear pairing given in Proposition 80 in [FL1].

Proof. The result follows by identifying the functions listed in Proposition 60 that are dual to the $K$-types of $\left(\rho_{2}, \mathcal{Q}^{-}\right),\left(\rho_{2}, \mathcal{Q}^{+}\right)$and $\left(\rho_{2}, \mathcal{Q}^{0}\right)$ respectively via the bilinear pairing given in Proposition 80 in [FL1].

Combining this with our description of irreducible components of $\left(\rho_{2}^{\prime}\right.$, ker Mx $)$, we obtain a complete list of all thirteen irreducible components of $\left(\rho_{2}^{\prime}, \mathcal{W}^{\prime}\right)$. Now that we know the irreducible components of $\left(\rho_{2}, \mathcal{W}\right)$ and $\left(\rho_{2}^{\prime}, \mathcal{W}^{\prime}\right)$, it is easy to identify the indecomposable subrepresentations of $\left(\rho_{2}, \mathcal{W}\right)$ and $\left(\rho_{2}^{\prime}, \mathcal{W}^{\prime}\right)$.

Collecting the $K$-types of each irreducible components $\left(\rho_{2}^{\prime}, \mathcal{W}^{\prime}\right)$, we obtain a decomposition of $\mathcal{W}^{\prime}$ into a direct sum of thirteen $K$-invariant vector subspaces. This decomposition is compatible with the decomposition of $\left(\rho_{1}, \mathscr{W}\right)$ into irreducible components

$$
\left(\rho_{1}, \mathscr{W}\right)=\left(\rho_{1}, \mathscr{K}^{+}\right) \oplus\left(\rho_{1}, \mathscr{K}^{0}\right) \oplus\left(\rho_{1}, \mathscr{K}^{-}\right)
$$

given in Section 4 of [FL3] in the following sense. 
Lemma 63. As vector spaces, $\mathbb{H}_{\mathbb{C}} \otimes \mathscr{W}^{\prime}=\mathcal{W}^{\prime}=\left(\mathbb{H}_{\mathbb{C}} \otimes \mathscr{K}^{+}\right) \oplus\left(\mathbb{H}_{\mathbb{C}} \otimes \mathscr{W}^{0}\right) \oplus\left(\mathbb{H}_{\mathbb{C}} \otimes \mathscr{T}^{-}\right)$, and $\mathbb{H}_{\mathbb{C}} \otimes \mathcal{K}^{+}=\partial^{+}\left(\mathcal{B H}^{+} / \mathcal{I}_{0}\right) \oplus \partial^{+}\left(H^{+} / \mathcal{B H}^{+}\right) \oplus\left(\mathcal{W}^{\prime} / \operatorname{ker} \tau_{a}^{+}\right) \oplus\left(\mathcal{W}^{\prime} / \operatorname{ker} \tau_{s}^{+}\right) \oplus \mathcal{Q}^{\prime+}$, $\mathbb{H}_{\mathbb{C}} \otimes \mathcal{K}^{0}=\partial^{+}\left(\mathcal{B H}^{-} / \mathcal{I}_{0}\right) \oplus \partial^{+}\left(\mathcal{J}^{\prime} /\left(\mathcal{B H}^{+}+\mathcal{B H} \mathcal{H}^{-}\right)\right) \oplus \mathbb{C}$-span of $\left\{N(Z)^{-1} \cdot Z\right\}$

$$
\oplus\left(\mathcal{W}^{\prime} / \operatorname{ker} \tau_{a}^{-}\right) \oplus\left(\mathcal{W}^{\prime} / \operatorname{ker} \tau_{s}^{-}\right) \oplus \mathcal{Q}^{\prime 0}
$$

$\mathbb{H}_{\mathbb{C}} \otimes \mathscr{W}^{-}=\partial^{+}\left(H^{\prime-} / \mathcal{B H}^{-}\right) \oplus \mathcal{Q}^{\prime-}$.

On the other hand, the image of $\mathrm{Mx}: \mathcal{W}^{\prime} \rightarrow \mathcal{W}$ has three irreducible components $\left(\rho_{2}, \mathcal{Q}^{+}\right)$, $\left(\rho_{2}, \mathcal{Q}^{0}\right),\left(\rho_{2}, \mathcal{Q}^{-}\right)$, and

$$
\mathcal{Q}^{+} \subset \mathbb{H}_{\mathbb{C}} \otimes \mathscr{K}^{+}, \quad \mathcal{Q}^{0} \subset \mathbb{H}_{\mathbb{C}} \otimes \mathscr{K}^{0}, \quad \mathcal{Q}^{-} \subset \mathbb{H}_{\mathbb{C}} \otimes \mathscr{K}^{-} .
$$

\section{Polarization of Vacuum and Equivariant Maps $\left(\rho_{2}^{\prime}, \mathcal{W}^{\prime}\right) \rightarrow\left(\pi_{l} \otimes\right.$} $\pi_{r}, \widehat{\mathcal{V} \otimes \mathcal{V}^{\prime}}$

\subsection{Equivariant $\operatorname{Maps}\left(\rho_{2}^{\prime}, \mathcal{W}^{\prime}\right) \rightarrow\left(\pi_{l} \otimes \pi_{r}, \widetilde{\mathcal{V} \otimes \mathcal{V}^{\prime}}\right)$}

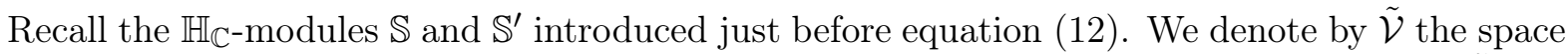
of $\mathbb{S}$-valued holomorphic left regular functions on $\mathbb{H}_{\mathbb{C}}$ (possibly with singularities) and by $\tilde{\mathcal{V}}^{\prime}$ the space of $\mathbb{S}^{\prime}$-valued holomorphic right regular functions on $\mathbb{H}_{\mathbb{C}}$ (possibly with singularities). The group $G L\left(2, \mathbb{H}_{\mathbb{C}}\right)$ acts on these spaces via

$$
\begin{aligned}
& \pi_{l}(h): f(Z) \quad \mapsto \quad\left(\pi_{l}(h) f\right)(Z)=\frac{(c Z+d)^{-1}}{N(c Z+d)} \cdot f\left((a Z+b)(c Z+d)^{-1}\right), \\
& \pi_{r}(h): g(Z) \quad \mapsto \quad\left(\pi_{r}(h) g\right)(Z)=g\left(\left(a^{\prime}-Z c^{\prime}\right)^{-1}\left(-b^{\prime}+Z d^{\prime}\right)\right) \cdot \frac{\left(a^{\prime}-Z c^{\prime}\right)^{-1}}{N\left(a^{\prime}-Z c^{\prime}\right)}
\end{aligned}
$$

respectively, where $f \in \tilde{\mathcal{V}}, g \in \tilde{\mathcal{V}}^{\prime}, h=\left(\begin{array}{ll}a^{\prime} & b^{\prime} \\ c^{\prime} & d^{\prime}\end{array}\right) \in G L\left(2, \mathbb{H}_{\mathbb{C}}\right)$ and $h^{-1}=\left(\begin{array}{ll}a & b \\ c & d\end{array}\right)$. Inside $\tilde{\mathcal{V}}$ and $\tilde{\mathcal{V}}^{\prime}$, we have subspaces

$$
\begin{aligned}
\mathcal{V}^{+} & =\left\{f \in \tilde{\mathcal{V}} ; f: \mathbb{H}_{\mathbb{C}} \rightarrow \mathbb{S} \text { is a polynomial map }\right\}, \\
\mathcal{V}^{-} & =\left\{f \in \tilde{\mathcal{V}} ; \pi_{l}\left(\begin{array}{ll}
0 & 1 \\
1 & 0
\end{array}\right) f=N(Z)^{-1} \cdot Z^{-1} \cdot f\left(Z^{-1}\right) \in \mathcal{V}^{+}\right\}, \\
\mathcal{V}^{\prime+} & =\left\{g \in \tilde{\mathcal{V}}^{\prime} ; g: \mathbb{H}_{\mathbb{C}} \rightarrow \mathbb{S}^{\prime} \text { is a polynomial map }\right\}, \\
\mathcal{V}^{\prime-} & =\left\{g \in \tilde{\mathcal{V}}^{\prime} ; \pi_{r}\left(\begin{array}{ll}
0 & 1 \\
1 & 0
\end{array}\right) g=-N(Z)^{-1} \cdot g\left(Z^{-1}\right) \cdot Z^{-1} \in \mathcal{V}^{\prime+}\right\} .
\end{aligned}
$$

We can form a tensor product representation $\left(\pi_{l} \otimes \pi_{r}, \tilde{\mathcal{V}} \otimes \tilde{\mathcal{V}}^{\prime}\right)$ and consider a larger space

$$
\widetilde{\mathcal{V} \otimes \mathcal{V}^{\prime}}=\left\{\begin{array}{c}
\text { holomorphic } \mathbb{H}_{\mathbb{C}^{-} \text {valued functions in two variables }} \\
Z_{1}, Z_{2} \in \mathbb{H}_{\mathbb{C}}(\text { possibly with singularities) that are left regular } \\
\text { with respect to } Z_{1} \text { and right regular with respect to } Z_{2}
\end{array}\right\} .
$$

The action of $G L\left(2, \mathbb{H}_{\mathbb{C}}\right)$ on these functions is given by

$$
\begin{aligned}
& \left(\pi_{l} \otimes \pi_{r}\right)(h): F\left(Z_{1}, Z_{2}\right) \quad \mapsto \quad\left(\left(\pi_{l} \otimes \pi_{r}\right)(h) F\right)\left(Z_{1}, Z_{2}\right) \\
& \quad=\frac{\left(c Z_{1}+d\right)^{-1}}{N\left(c Z_{1}+d\right)} \cdot F\left(\left(a Z_{1}+b\right)\left(c Z_{1}+d\right)^{-1},\left(a^{\prime}-Z_{2} c^{\prime}\right)\left(-b^{\prime}+Z_{2} d^{\prime}\right)^{-1}\right) \cdot \frac{\left(a^{\prime}-Z_{2} c^{\prime}\right)^{-1}}{N\left(a^{\prime}-Z_{2} c^{\prime}\right)}
\end{aligned}
$$

Differentiating, we obtain the corresponding action of the Lie algebra $\mathfrak{g l}\left(2, \mathbb{H}_{\mathbb{C}}\right)$ also denoted by $\pi_{l} \otimes \pi_{r}$. 
We denote by Diag the restriction to the diagonal map

$$
\text { Diag : } F\left(Z_{1}, Z_{2}\right) \mapsto F(Z, Z) \text {. }
$$

Clearly, Diag intertwines the actions of $\pi_{l} \otimes \pi_{r}$ and $\rho_{2}$.

We consider maps $\mathcal{W}^{\prime} \rightarrow \widehat{\mathcal{V} \otimes \mathcal{V}^{\prime}}$

$$
\left(J_{R} F\right)\left(Z_{1}, Z_{2}\right)=\frac{12 i}{\pi^{3}} \int_{W \in U(2)_{R}} \frac{\left(W-Z_{1}\right)^{-1}}{N\left(W-Z_{1}\right)} \cdot F(W) \cdot \frac{\left(W-Z_{2}\right)^{-1}}{N\left(W-Z_{2}\right)} d V .
$$

If $Z_{1}, Z_{2} \in \mathbb{D}_{R}^{-} \sqcup \mathbb{D}_{R}^{+}$, the integrand has no singularities and the result is a holomorphic function in two variables $Z_{1}, Z_{2}$ which is harmonic in each variable separately. We will see soon that the result depends on whether $Z_{1}$ and $Z_{2}$ are both in $\mathbb{D}_{R}^{+}$, both in $\mathbb{D}_{R}^{-}$or one is in $\mathbb{D}_{R}^{+}$and the other is in $\mathbb{D}_{R}^{-}$. Thus the expression (57) determines four different maps. We use notations $J_{R}^{++}$and $J_{R}^{--}$to signify $Z_{1}, Z_{2} \in \mathbb{D}_{R}^{+}$and $Z_{1}, Z_{2} \in \mathbb{D}_{R}^{-}$respectively. (Notations $J_{R}^{+-}$and $J_{R}^{-+}$will be introduced in the next subsection.) These maps $J_{R}$ are closely related to the maps $I_{R}$ given by equation (34) in Chapter 6 of [FL3]

$$
\mathscr{W} \ni f \quad \mapsto \quad\left(I_{R} f\right)\left(Z_{1}, Z_{2}\right)=\frac{i}{2 \pi^{3}} \int_{W \in U(2)_{R}} \frac{f(W) d V}{N\left(W-Z_{1}\right) \cdot N\left(W-Z_{2}\right)} \in \widetilde{\mathcal{H} \otimes \mathcal{H}}
$$

where $\widetilde{\mathcal{H} \otimes \mathcal{H}}$ denotes the space of holomorphic $\mathbb{C}$-valued functions in two variables $Z_{1}, Z_{2} \in \mathbb{H}_{\mathbb{C}}$ (possibly with singularities) that are harmonic in each variable separately. Indeed, $I_{R}$ extends to a map on $\mathbb{H}_{\mathbb{C}} \otimes \mathscr{T}=\mathcal{W}^{\prime}$, and

$$
J_{R} F\left(Z_{1}, Z_{2}\right)=24 \vec{\partial}_{Z_{1}}\left(I_{R} F\left(Z_{1}, Z_{2}\right)\right) \overleftarrow{\partial}_{Z_{2}}
$$

Recall from Section 2 of [FL3] that the group $U(2,2)_{R}$ is a conjugate of $U(2,2)$, which is a real form of $G L\left(2, \mathbb{H}_{\mathbb{C}}\right)$ preserving $U(2)_{R}, \mathbb{D}_{R}^{+}$and $\mathbb{D}_{R}^{-}$.

Proposition 64. The maps $F \mapsto\left(J_{R} F\right)\left(Z_{1}, Z_{2}\right)$ are $U(2,2)_{R}$ and $\mathfrak{g l}\left(2, \mathbb{H}_{\mathbb{C}}\right)$-equivariant maps from $\left(\rho_{2}^{\prime}, \mathcal{W}^{\prime}\right)$ to $\left(\pi_{l} \otimes \pi_{r}, \widehat{\mathcal{V} \otimes \mathcal{V}^{\prime}}\right)$.

Proof. We need to show that, for all $h \in U(2,2)_{R}$, the maps (57) commute with the action of $h$. Writing $h=\left(\begin{array}{ll}a^{\prime} & b^{\prime} \\ c^{\prime} & d^{\prime}\end{array}\right), h^{-1}=\left(\begin{array}{ll}a & b \\ c & d\end{array}\right)$,

$$
\tilde{Z}_{1}=\left(a Z_{1}+b\right)\left(c Z_{1}+d\right)^{-1}, \quad \tilde{Z}_{2}=\left(a Z_{2}+b\right)\left(c Z_{2}+d\right)^{-1}, \quad \tilde{W}=(a W+b)(c W+d)^{-1}
$$

and using Lemmas 10 and 61 from [FL1] we obtain:

$$
\begin{gathered}
\int_{W \in U(2)_{R}} \frac{\left(W-Z_{1}\right)^{-1}}{N\left(W-Z_{1}\right)} \cdot\left(\rho_{2}^{\prime}(h) F\right)(W) \cdot \frac{\left(W-Z_{2}\right)^{-1}}{N\left(W-Z_{2}\right)} d V \\
=\int_{W \in U(2)_{R}} \frac{\left(W-Z_{1}\right)^{-1}}{N\left(W-Z_{1}\right)} \cdot \frac{\left(a^{\prime}-W c^{\prime}\right)}{N\left(a^{\prime}-W c^{\prime}\right)} \cdot F(\tilde{W}) \cdot \frac{(c W+d)}{N(c W+d)} \cdot \frac{\left(W-Z_{2}\right)^{-1}}{N\left(W-Z_{2}\right)} d V \\
=\int_{W \in U(2)_{R}} \frac{\left(c Z_{1}+d\right)^{-1} \cdot\left(W-\tilde{Z}_{1}\right)^{-1} \cdot F(\tilde{W}) \cdot\left(\tilde{W}-\tilde{Z}_{2}\right)^{-1} \cdot\left(a^{\prime}-Z_{2} c^{\prime}\right)^{-1} d V}{N\left(c Z_{1}+d\right) \cdot N\left(a^{\prime}-W c^{\prime}\right)^{2} \cdot N\left(\tilde{W}-\tilde{Z}_{1}\right) \cdot N\left(\tilde{W}-\tilde{Z}_{2}\right) \cdot N(c W+d)^{2} \cdot N\left(a^{\prime}-Z_{2} c^{\prime}\right)} \\
=\frac{\left(c Z_{1}+d\right)^{-1}}{N\left(c Z_{1}+d\right)} \cdot \int_{\tilde{W} \in U(2)_{R}} \frac{\left(\tilde{W}-\tilde{Z}_{1}\right)^{-1}}{N\left(\tilde{W}-\tilde{Z}_{1}\right)} \cdot F(\tilde{W}) \cdot \frac{\left(\tilde{W}_{2}-\tilde{Z}_{2}\right)^{-1}}{N\left(\tilde{W}-\tilde{Z}_{2}\right)} d V \cdot \frac{\left(a^{\prime}-Z_{2} c^{\prime}\right)^{-1}}{N\left(a^{\prime}-Z_{2} c^{\prime}\right)} .
\end{gathered}
$$

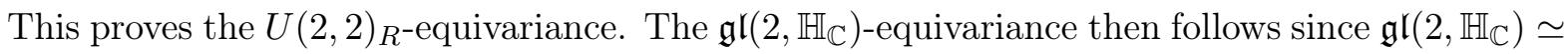
$\mathbb{C} \otimes \mathfrak{u}(2,2)_{R}$. 
We compose the maps $J_{R}$ with the restriction onto the diagonal map Diag defined by (56). Note that the subspace $\mathcal{V}^{+} \otimes \mathcal{V}^{\prime+} \subset \widetilde{\mathcal{V} \otimes \mathcal{V}^{\prime}}$ can be described as the $\mathbb{H}_{\mathbb{C}}$-valued polynomials in $\widehat{\mathcal{V} \otimes \mathcal{V}^{\prime}}$.

Theorem 65. The maps $F \mapsto\left(J_{R} F\right)\left(Z_{1}, Z_{2}\right)$ have the following properties:

1. If $Z_{1}, Z_{2} \in \mathbb{D}_{R}^{+}$, then $J_{R}^{++}$maps $\mathcal{W}^{\prime}$ into $\mathcal{V}^{+} \otimes \mathcal{V}^{++} \subset \widetilde{\mathcal{V} \otimes \mathcal{V}^{\prime}}$, annihilates all irreducible components of $\left(\rho_{2}^{\prime}, \mathcal{W}^{\prime}\right)$, except for $\mathcal{Q}^{\prime+}$, and

$$
\operatorname{Diag} \circ\left(J_{R}^{++} F\right)\left(Z_{1}, Z_{2}\right)=\mathrm{Mx} F, \quad \text { if } F \in \mathbb{H}_{\mathbb{C}} \otimes \mathscr{K}^{+} .
$$

2. If $Z_{1}, Z_{2} \in \mathbb{D}_{R}^{-}$, then $J_{R}^{--}$maps $\mathcal{W}^{\prime}$ into $\mathcal{V}^{-} \otimes \mathcal{V}^{-} \subset \widetilde{\mathcal{V} \otimes \mathcal{V}^{\prime}}$, annihilates all irreducible components of $\left(\rho_{2}^{\prime}, \mathcal{W}^{\prime}\right)$, except for $\mathcal{Q}^{\prime-}$, and

$$
\operatorname{Diag} \circ\left(J_{R}^{--} F\right)\left(Z_{1}, Z_{2}\right)=\mathrm{Mx} F, \quad \text { if } F \in \mathbb{H}_{\mathbb{C}} \otimes \mathscr{K}^{-} .
$$

Proof. We prove part 1 only, the other part can be proven in the same way. So, suppose that $Z_{1}, Z_{2} \in \mathbb{D}_{R}^{+}$. It follows immediately from Theorem 12 in [FL3], Lemma 63] and equation (59) that the image of $J_{R}^{++}$lies in $\mathcal{V}^{+} \otimes \mathcal{V}^{\prime+}$ and that $J_{R}^{++}$annihilates the irreducible components of $\left(\rho_{2}^{\prime}, \mathcal{W}^{\prime}\right)$ that lie in $\mathbb{H}_{\mathbb{C}} \otimes\left(\mathscr{K}^{0} \oplus \mathscr{K}^{-}\right)$(as described in Lemma 63). Then we check the effect of $J_{R}^{++}$on a suitable generator of each of the remaining irreducible component of $\left(\rho_{2}^{\prime}, \mathcal{W}^{\prime}\right)$. For the irreducible components from Corollary 51

$$
\left(\rho_{2}^{\prime}, \mathcal{W}^{\prime} / \operatorname{ker} \tau_{a}^{+}\right) \quad \text { and } \quad\left(\rho_{2}^{\prime}, \mathcal{W}^{\prime} / \operatorname{ker} \tau_{s}^{+}\right),
$$

choose generators $\tilde{F}_{\frac{1}{2}, \frac{1}{2},-\frac{1}{2}}(W)$ and $\tilde{G}_{\frac{1}{2},-\frac{1}{2}, \frac{1}{2}}(W)$ respectively. The image of $\mathrm{HI}^{+}$under $\partial^{+}$is generated by $\partial^{+}\left(N(Z)^{2}\right)=2 N(Z) \cdot Z$ and contains two irreducible components. We show the calculations for $N(Z) \cdot Z$ only, the other cases are similar. Using Lemma 23 and the matrix coefficient expansion of $\frac{\left(W-Z_{1}\right)^{-1}}{N\left(W-Z_{1}\right)}$ given by Proposition 26 in [FL1] (see also Proposition 113), we compute:

$$
\begin{aligned}
& J_{R}^{++}(N(W) \cdot W)\left(Z_{1}, Z_{2}\right)=\frac{24 i}{\pi^{3}} \int_{W \in U(2)_{R}} \frac{\left(W-Z_{1}\right)^{-1}}{N\left(W-Z_{1}\right)} \cdot N(W) \cdot W \cdot \frac{\left(W-Z_{2}\right)^{-1}}{N\left(W-Z_{2}\right)} d V \\
& =\frac{24 i}{\pi^{3}} \sum_{l, m, n, l^{\prime}, m^{\prime}, n^{\prime}}\left(\begin{array}{c}
\left(l-m+\frac{1}{2}\right) t^{l}{ }_{n \rightarrow+\frac{1}{2}}\left(Z_{1}\right) \\
\left(l+m+\frac{1}{2}\right) t_{n \underline{m-\frac{1}{2}}}^{l}\left(Z_{1}\right)
\end{array}\right) \\
& \times \int_{W \in U(2)_{R}}\left(t_{m \underline{n-\frac{1}{2}}}^{l+\frac{1}{2}}\left(W^{-1}\right), t_{m \underline{n+\frac{1}{2}}}^{l+\frac{1}{2}}\left(W^{-1}\right)\right) \cdot \frac{W}{N(W)} \cdot\left(\begin{array}{c}
\left(l^{\prime}-n^{\prime}+\frac{1}{2}\right) t_{n^{\prime}-\frac{1}{2} \underline{m^{\prime}}} \\
\left(l^{\prime}+n^{\prime}+\frac{1}{2}\right) t_{n^{\prime}+\frac{1}{2} \underline{\underline{m}^{\prime}}}
\end{array}\right. \\
& \times\left(t_{m^{\prime}+\frac{1}{2} \underline{\underline{n}^{\prime}}}^{l^{\prime}-\frac{1}{2}}\left(Z_{2}\right), t_{m^{\prime}-\frac{1}{2} \underline{\underline{h}^{\prime}}}^{l^{\prime}-\frac{1}{2}}\left(Z_{2}\right)\right)
\end{aligned}
$$

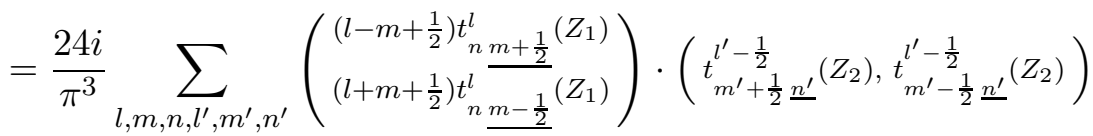

$$
\begin{aligned}
& \times \int_{W \in U(2)_{R}} \frac{1}{N(W)}\left(t_{m+\frac{1}{2} \underline{\underline{n}}}^{l}\left(W^{-1}\right), t_{m-\frac{1}{2} \underline{\underline{n}}}^{l}\left(W^{-1}\right)\right) \cdot\left(\begin{array}{c}
\left(l^{\prime}-n^{\prime}+\frac{1}{2}\right) t_{n^{\prime}-\frac{1}{2} \underline{\underline{m}}^{\prime}} \\
\left(l^{\prime}+n^{\prime}+\frac{1}{2}\right) t_{n^{\prime}+\frac{1}{2} \underline{\underline{m}}^{\prime}}\left(W^{-1}\right)
\end{array}\right) d V=0
\end{aligned}
$$

by the orthogonality relations (19) in [FL3], since $l^{\prime} \geq 1 / 2$. We conclude from Proposition 64 that $J_{R}^{++}$annihilates all of $\left(\rho_{2}^{\prime}, \partial^{+}\left(H^{+}\right)\right)$.

Finally, the statement about the composition Diag $\circ J_{R}^{++}$follows from Theorem 77 in [FL1]. (Note that the differential form $d V$ that appears in (57) differs from $d Z^{4}$ that appears in Theorem 77 in [FL1 by a factor of $1 / 4$.) 


\subsection{Polarization of Vacuum}

Now we suppose $Z_{1} \in \mathbb{D}_{R}^{+}$and $Z_{2} \in \mathbb{D}_{R}^{-}$(or the other way around), this case is much more subtle. We reduce the spinor case of $J_{R}$ to the already known scalar case of $I_{R}$ as much as possible via the relation (59).

Proposition 66. Let $Z_{1} \in \mathbb{D}_{R}^{+}$and $Z_{2} \in \mathbb{D}_{R}^{-}$, then the map $J_{R}$ annihilates all irreducible components of $\left(\rho_{2}^{\prime}, \mathcal{W}^{\prime}\right)$, except for $\mathcal{Q}^{\prime 0}$ and the trivial one-dimensional representation spanned by $N(W)^{-1} \cdot W$. Moreover,

$$
J_{R}\left(N(W)^{-1} \cdot W\right)\left(Z_{1}, Z_{2}\right)=24 \frac{\left(Z_{1}-Z_{2}\right)^{-1}}{N\left(Z_{1}-Z_{2}\right)} .
$$

Similarly, if $Z_{1} \in \mathbb{D}_{R}^{-}$and $Z_{2} \in \mathbb{D}_{R}^{+}$, then the map $J_{R}$ annihilates all irreducible components of $\left(\rho_{2}^{\prime}, \mathcal{W}^{\prime}\right)$, except for $\mathcal{Q}^{\prime 0}$ and the trivial one-dimensional representation spanned by $N(W)^{-1} \cdot W$. Moreover,

$$
J_{R}\left(N(W)^{-1} \cdot W\right)\left(Z_{1}, Z_{2}\right)=-24 \frac{\left(Z_{1}-Z_{2}\right)^{-1}}{N\left(Z_{1}-Z_{2}\right)} .
$$

Proof. Since $I_{R}$ annihilates $\mathscr{K}^{+} \oplus \mathscr{K}^{-}$, it follows from equation (59) that $J_{R}$ annihilates the irreducible components of $\left(\rho_{2}^{\prime}, \mathcal{W}^{\prime}\right)$ that lie in $\mathbb{H}_{\mathbb{C}} \otimes\left(\mathscr{K}^{+} \oplus \mathscr{K}^{-}\right)$(as described in Lemma 63). Then we check the effect of $J_{R}$ on a suitable generator of each of the remaining irreducible component of $\left(\rho_{2}^{\prime}, \mathcal{W}^{\prime}\right)$.

The image of $\amalg^{\prime}$ under $\partial^{+}$is generated by two generators

$$
\partial^{+}\left(N(Z)^{2}\right)=2 N(Z) \cdot Z \quad \text { and } \quad \partial^{+}\left(N(Z)^{-2}\right)=-2 N(Z)^{-3} \cdot Z
$$

these take care of irreducible components contained in $\partial^{+}\left(H^{\prime}\right)$. For the irreducible components $\left(\rho_{2}^{\prime}, \mathcal{W}^{\prime} / \operatorname{ker} \tau_{a}^{-}\right)$and $\left(\rho_{2}^{\prime}, \mathcal{W}^{\prime} / \operatorname{ker} \tau_{s}^{-}\right)$make a choice of generators such as

$$
\tilde{F}_{\frac{1}{2}, \frac{1}{2},-\frac{1}{2}}^{\prime}(W)=\frac{2}{N(W)^{2}}\left(\begin{array}{cc}
0 & 0 \\
w_{11} & w_{12}
\end{array}\right) \quad \text { and } \quad \tilde{G}_{\frac{1}{2},-\frac{1}{2}, \frac{1}{2}}^{\prime}(W)=\frac{1}{N(W)^{2}}\left(\begin{array}{cc}
0 & w_{11} \\
0 & w_{21}
\end{array}\right)
$$

respectively. We show the calculations for $\tilde{F}_{\frac{1}{2}, \frac{1}{2},-\frac{1}{2}}^{\prime}(W)$ with $Z_{1} \in \mathbb{D}_{R}^{+}, Z_{2} \in \mathbb{D}_{R}^{-}$only; the other cases are similar and easier. Using Lemma 23 and the matrix coefficient expansion of $\frac{\left(W-Z_{1}\right)^{-1}}{N\left(W-Z_{1}\right)}$ given by Proposition 26 in [FL1] (see also Proposition 113), we compute:

$$
\begin{aligned}
& J_{R}\left(\tilde{F}_{\frac{1}{2}, \frac{1}{2},-\frac{1}{2}}^{\prime}(W)\right)\left(Z_{1}, Z_{2}\right) \\
& =\frac{24 i}{\pi^{3}} \int_{W \in U(2)_{R}} \frac{\left(W-Z_{1}\right)^{-1}}{N\left(W-Z_{1}\right)} \cdot \frac{1}{N(W)^{2}} \cdot\left(\begin{array}{cc}
0 & 0 \\
w_{11} & w_{12}
\end{array}\right) \cdot \frac{\left(W-Z_{2}\right)^{-1}}{N\left(W-Z_{2}\right)} d V \\
& =-\frac{24 i}{\pi^{3}} \sum_{l, m, n, l^{\prime}, m^{\prime}, n^{\prime}}\left(\begin{array}{c}
\left(l-m+\frac{1}{2}\right) t_{n}^{l}{ }_{n+\frac{1}{2}}\left(Z_{1}\right) \\
\left(l+m+\frac{1}{2}\right) t_{n \underline{n-\frac{1}{2}}}^{l} \\
\left(Z_{1}\right)
\end{array}\right)
\end{aligned}
$$

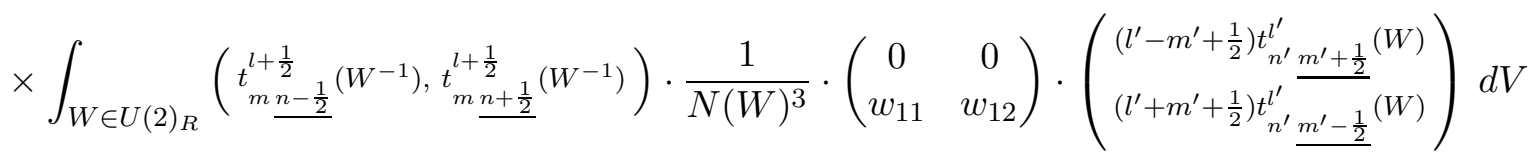

$$
\begin{aligned}
& \times N\left(Z_{2}\right)^{-1} \cdot\left(t_{m^{\prime} \underline{n^{\prime}-\frac{1}{2}}}^{l^{\prime}+\frac{1}{2}}\left(Z_{2}^{-1}\right), t_{m^{\prime} \underline{n^{\prime}+\frac{1}{2}}}^{l^{\prime}+\frac{1}{2}}\left(Z_{2}^{-1}\right)\right)
\end{aligned}
$$

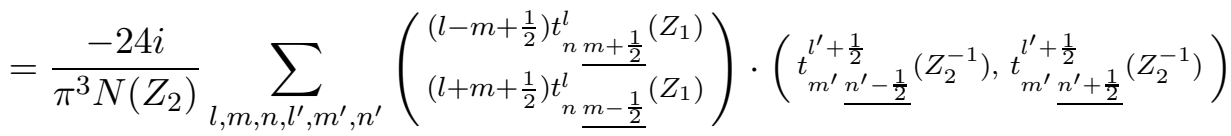

$$
\begin{aligned}
& \times \int_{W \in U(2)_{R}}\left(t_{m \underline{n-\frac{1}{2}}}^{l+\frac{1}{2}}\left(W^{-1}\right), t_{m \underline{n+\frac{1}{2}}}^{l+\frac{1}{2}}\left(W^{-1}\right)\right) \cdot \frac{1}{N(W)^{3}} \cdot\left(\begin{array}{c}
0 \\
\left(l^{\prime}-n^{\prime}+1\right) t_{n^{\prime}-\frac{1}{2} \underline{m}^{\prime}}^{l^{\prime}+\frac{1}{2}}
\end{array}\right) d V=0
\end{aligned}
$$


by the orthogonality relations (19) in [FL3], since the power of $N(W)$ is not -2 . We conclude from Proposition 64 that $J_{R}$ annihilates all of $\left(\rho_{2}^{\prime}, \mathcal{W}^{\prime} / \operatorname{ker} \tau_{a}^{-}\right)$.

For $Z_{1}, Z_{2} \in \mathbb{H}_{\mathbb{C}}^{\times}$, let $\lambda_{1}$ and $\lambda_{2}$ denote the eigenvalues of $Z_{1} Z_{2}^{-1}$, and introduce an open subset of $\mathbb{H}_{\mathbb{C}}^{\times} \times \mathbb{H}_{\mathbb{C}}^{\times}$

$$
\Omega_{0}=\left\{\left(Z_{1}, Z_{2}\right) \in \mathbb{H}_{\mathbb{C}}^{\times} \times \mathbb{H}_{\mathbb{C}}^{\times} ; \begin{array}{c}
\lambda_{1} \neq 1, \lambda_{2} \neq 1, \text { neither } \frac{1-\lambda_{1}}{1-\lambda_{2}} \text { nor } \\
\frac{1-\lambda_{1}^{-1}}{1-\lambda_{2}^{-1}} \text { is a negative real number }
\end{array}\right\} .
$$

Let $Z_{1} \in \mathbb{D}_{R}^{+}$and $Z_{2} \in \mathbb{D}_{R}^{-}$and recall the relation (59) between $J_{R}$ and $I_{R}$. From Section 6 of [FL3], we see that, for any $F \in \mathcal{W}^{\prime},\left(J_{R} F\right)\left(Z_{1}, Z_{2}\right)$ extends analytically across $\Omega_{0}$, and we have a well defined operator $J_{R}^{+-}$on $\mathcal{W}^{\prime}$ :

$$
\left(J_{R}^{+-} F\right)\left(Z_{1}, Z_{2}\right)=\text { analytic extension of }\left(J_{R} F\right)\left(Z_{1}, Z_{2}\right) \text { from } \mathbb{D}_{R}^{+} \times \mathbb{D}_{R}^{-} \text {to } \Omega_{0} \text {. }
$$

The operator $J_{R}^{+-}$is $U(2) \times U(2)$ and $\mathfrak{g l}\left(2, \mathbb{H}_{\mathbb{C}}\right.$ )-equivariant (which follows from Proposition 64) and annihilates all irreducible components of $\left(\rho_{2}^{\prime}, \mathcal{W}^{\prime}\right)$, except for $\mathcal{Q}^{\prime 0}$ and the trivial onedimensional representation spanned by $N(W)^{-1} \cdot W$ (which follows from Proposition 66). While $J_{R}^{+-} F$ is independent of the choice of $R>0$, we keep the subscript $R$ to distinguish this analytic function from a formal series $J^{+-} F$ that will be defined in Subsection 7.3 .

Similarly, we can switch the domains of $Z_{1}$ and $Z_{2}$ and define another operator $J_{R}^{-+}$on $\mathcal{W}^{\prime}$ :

$$
\left(J_{R}^{-+} F\right)\left(Z_{1}, Z_{2}\right)=\text { analytic extension of }\left(J_{R} F\right)\left(Z_{1}, Z_{2}\right) \text { from } \mathbb{D}_{R}^{-} \times \mathbb{D}_{R}^{+} \text {to } \Omega_{0} \text {. }
$$

The operator $J_{R}^{-+}$is also $U(2) \times U(2)$ and $\mathfrak{g l}\left(2, \mathbb{H}_{\mathbb{C}}\right)$-equivariant and annihilates all irreducible components of $\left(\rho_{2}^{\prime}, \mathcal{W}^{\prime}\right)$, except for $\mathcal{Q}^{\prime 0}$ and the trivial one-dimensional representation spanned by $N(W)^{-1} \cdot W$.

We introduce the following notation: if $\lambda \in \mathbb{C}$, let

$$
\operatorname{sign}(\operatorname{Im} \lambda)= \begin{cases}1 & \text { if } \lambda \text { lies in the upper half plane of } \mathbb{C} \\ -1 & \text { if } \lambda \text { lies in the lower half plane of } \mathbb{C} \\ \text { undefined } & \text { if } \lambda \in \mathbb{R} .\end{cases}
$$

Theorem 67. We have a well defined operator on $\mathcal{W}^{\prime}$

$$
\left(\mathrm{Mx}^{0} F\right)(Z)=\lim _{\substack{Z_{1}, Z_{2} \rightarrow Z, N\left(Z_{1}-Z_{2}\right) \neq 0 \\ \operatorname{sign}\left(\operatorname{Im} \lambda_{1}\right)=\operatorname{sign}\left(\operatorname{Im} \lambda_{2}\right)}}-\left(\left(J_{R}^{+-}+J_{R}^{-+}\right) F\right)\left(Z_{1}, Z_{2}\right), \quad Z \in U(2)_{R}
$$

where $\lambda_{1}$ and $\lambda_{2}$ are the eigenvalues of $Z_{1} Z_{2}^{-1}$. The operator $\mathrm{Mx}^{0}$ has values in $\mathcal{W}$, is $\mathfrak{g l}\left(2, \mathbb{H}_{\mathbb{C}}\right)$ equivariant, annihilates all irreducible components of $\left(\rho_{2}^{\prime}, \mathcal{W}^{\prime}\right)$, except for $\mathcal{Q}^{\prime 0}$, and equals $\mathrm{Mx}$ on $\mathcal{Q}^{\prime 0}$.

Furthermore, the operator $\mathrm{Mx}^{0}$ on $\mathcal{W}^{\prime}$ can be computed as follows:

$$
\begin{aligned}
\left(\mathrm{Mx}^{0} F\right)(Z) & =-\frac{12 i}{\pi^{3}} \lim _{\theta \rightarrow 0} \lim _{s \rightarrow 1}\left(\int_{W \in U(2)_{R}} \frac{\left(W-s e^{i \theta} Z\right)^{-1}}{N\left(W-s e^{i \theta} Z\right)} \cdot F(W) \cdot \frac{\left(W-s^{-1} e^{-i \theta} Z\right)^{-1}}{N\left(W-s^{-1} e^{-i \theta} Z\right)} d V\right. \\
& \left.+\int_{W \in U(2)_{R}} \frac{\left(W-s^{-1} e^{i \theta} Z\right)^{-1}}{N\left(W-s^{-1} e^{i \theta} Z\right)} \cdot F(W) \cdot \frac{\left(W-s e^{-i \theta} Z\right)^{-1}}{N\left(W-s e^{-i \theta} Z\right)} d V\right), \quad Z \in U(2)_{R} .
\end{aligned}
$$

Note that the space $\mathcal{W}^{\prime}$ consists of rational functions, and rational functions on $\mathbb{H}_{\mathbb{C}}$ as well as analytic ones are completely determined by their values on $U(2)_{R}$. 
Proof. First, we show that the limit (60) exists. The map $\left(J_{R}^{+-}+J_{R}^{-+}\right)$is related to the map $\left(I_{R}^{+-}+I_{R}^{-+}\right)$from Theorem 15 in [FL3] via

$$
\left(\left(J_{R}^{+-}+J_{R}^{-+}\right) F\right)\left(Z_{1}, Z_{2}\right)=24 \vec{\partial}_{Z_{1}}\left(\left(I_{R}^{+-}+I_{R}^{-+}\right) F\left(Z_{1}, Z_{2}\right)\right) \overleftarrow{\partial}_{Z_{2}}
$$

which is essentially equation (59). We saw in the course of proof of Theorem 15 in [FL3] (see also Theorem 116) that the image of $\left(I_{R}^{+-}+I_{R}^{-+}\right)$is generated by

$$
\left(\left(I_{R}^{+-}+I_{R}^{-+}\right) N(W)^{-1}\right)\left(Z_{1}, Z_{2}\right)=-\frac{1}{N\left(Z_{2}\right)} \cdot \begin{cases}\frac{\log \lambda_{2}-\log \lambda_{1}}{\lambda_{2}-\lambda_{1}} & \text { if } \lambda_{1} \neq \lambda_{2} \\ \lambda^{-1} & \text { if } \lambda_{1}=\lambda_{2}=\lambda, \lambda \neq 1\end{cases}
$$

where log denotes the branch of logarithm with a cut along the positive real axis. If we restrict $\left(\left(I_{R}^{+-}+I_{R}^{-+}\right) N(W)^{-1}\right)\left(Z_{1}, Z_{2}\right)$ to the open set where $\operatorname{sign}\left(\operatorname{Im} \lambda_{1}\right)=\operatorname{sign}\left(\operatorname{Im} \lambda_{2}\right)$, we see that this restriction is

$$
\frac{1}{N\left(Z_{2}\right)} \cdot\left(\text { function of } \lambda_{1}, \lambda_{2} \in \mathbb{C} \text { that is holomorphic near }\left(\lambda_{1}, \lambda_{2}\right)=(1,1)\right) \text {. }
$$

Since the map $\left(I_{R}^{+-}+I_{R}^{-+}\right)$is $\mathfrak{g l}\left(2, \mathbb{H}_{\mathbb{C}}\right)$-equivariant, it follows that the same is true for $\left(\left(I_{R}^{+-}+\right.\right.$ $\left.\left.I_{R}^{-+}\right) F\right)\left(Z_{1}, Z_{2}\right)$ with any $F \in \mathcal{W}^{\prime}$. Therefore, the limit (60) exists.

Clearly, the operator $\mathrm{Mx}^{0}$ on $\mathcal{W}^{\prime}$ is $\mathfrak{g l}\left(2, \mathbb{H}_{\mathbb{C}}\right)$-equivariant and, by Proposition 66, annihilates all irreducible components of $\left(\rho_{2}^{\prime}, \mathcal{W}^{\prime}\right)$, except for $\mathcal{Q}^{\prime 0}$. It remains to show that $\mathrm{Mx}^{0}$ equals $\mathrm{Mx}$ on $\mathcal{Q}^{\prime 0}$ on one particular generator, then the other statements of the theorem follow immediately, including the part that the values of $\mathrm{Mx}^{0}$ lie in $\mathcal{W}$. For this purpose we choose a generator

$$
N(Z)^{-1}=N(Z)^{-1} \cdot \tilde{H}_{0,0,0}(Z)+N(Z)^{-1} \cdot \tilde{H}_{0,-1,-1}(Z) \quad \in \mathcal{Q}^{\prime 0} .
$$

Moreover, since $\mathrm{Mx}^{0}$ is $(U(2) \times U(2))$-equivariant, it is sufficient to show that $\mathrm{Mx}^{0} N(Z)^{-1}=$ $\operatorname{Mx} N(Z)^{-1}$ when $Z$ is diagonal. And since the limit (60) is known to exist, we can assume that $Z_{1}, Z_{2}$ are diagonal as well. We have:

$$
\operatorname{Mx}\left(N(Z)^{-1}\right)=4 N(Z)^{-2}+8 N(Z)^{-3} \cdot\left(Z^{+}\right)^{2}
$$

and

$$
\operatorname{Mx}\left(\frac{1}{N(Z)}\right)=\frac{4}{a^{2} d^{2}}\left(\begin{array}{cc}
1+2 d / a & 0 \\
0 & 1+2 a / d
\end{array}\right), \quad \text { if } Z=\left(\begin{array}{ll}
a & 0 \\
0 & d
\end{array}\right) .
$$

Suppose first that $Z_{1} \in \mathbb{D}_{R}^{+}$and $Z_{2} \in \mathbb{D}_{R}^{-}$. Recall from Section 6 of [FL3] that

$$
\left(I_{R} N(W)^{-1}\right)\left(Z_{1}, Z_{2}\right)=\sum_{l, m, n} \frac{1}{2 l+1} t_{n \underline{m}}^{l}\left(Z_{1}\right) \cdot N\left(Z_{2}\right)^{-1} \cdot t_{m \underline{n}}^{l}\left(Z_{2}^{-1}\right) .
$$

By (59),

$$
\begin{aligned}
& \left(J_{R} N(W)^{-1}\right)\left(Z_{1}, Z_{2}\right)=24 \vec{\partial}_{Z_{1}}\left(\left(I_{R} N(W)^{-1}\right)\left(Z_{1}, Z_{2}\right)\right) \overleftarrow{\partial}_{Z_{2}} \\
& =-\sum_{l, m, n} \frac{24}{2 l+1}\left(\begin{array}{ll}
(l-m) t_{n+\frac{1}{2}}^{l-\frac{1}{2}}\left(Z_{1}\right) & (l-m) t_{n-\frac{1}{2}}^{l-\frac{1}{2}}\left(Z_{1+\frac{1}{2}}\right. \\
(l+m) t_{n+\frac{1}{2} \underline{m-\frac{1}{2}}}^{l-\frac{1}{2}}\left(Z_{1}\right) & \left.(l+m) t_{n-\frac{1}{2} \underline{m-\frac{1}{2}}}^{l-Z_{1}}\right)
\end{array}\right) \\
& \times \frac{1}{N\left(Z_{2}\right)}\left(\begin{array}{cc}
(l-m+1) t_{m-\frac{1}{2}}^{l+\frac{1}{2}}\left(Z_{2}^{-1}\right) & (l-m+1) t_{m-\frac{1}{2}}^{l+\frac{1}{2}}\left(Z_{\frac{1}{2}}^{-1}\right) \\
(l+m+1) t_{m+\frac{1}{2} \underline{n-\frac{1}{2}}}^{l+\frac{1}{2}}\left(Z_{2}^{-1}\right) & (l+m+1) t_{m+\frac{1}{2} \underline{n+\frac{1}{2}}}^{l+\frac{1}{2}}\left(Z_{2}^{-1}\right)
\end{array}\right) .
\end{aligned}
$$


Additionally, assume that $Z_{1}$ and $Z_{2}$ are diagonal: $Z_{1}=\left(\begin{array}{cc}a_{1} & 0 \\ 0 & d_{1}\end{array}\right)$ with $\left|a_{1}\right|,\left|d_{1}\right|<R$ and $Z_{2}=$ $\left(\begin{array}{cc}a_{2} & 0 \\ 0 & d_{2}\end{array}\right)$ with $\left|a_{2}\right|,\left|d_{2}\right|>R$, then

$$
t_{n \underline{m}}^{l}\left(\begin{array}{ll}
a & 0 \\
0 & d
\end{array}\right)= \begin{cases}a^{l-m} d^{l+m} & \text { if } m=n ; \\
0 & \text { otherwise, }\end{cases}
$$

and only the terms with $n=m-1, m, m+1$ are non-zero, and it is easy to see that

$$
\begin{aligned}
& \left(J_{R} N(W)^{-1}\right)\left(Z_{1}, Z_{2}\right) \\
& =\sum_{l, m} \frac{24}{2 l+1}\left(\begin{array}{cc}
\frac{\partial^{2}}{\partial a_{1} \partial a_{2}}+\frac{\partial^{2}}{\partial a_{1} \partial d_{2}} & 0 \\
0 & \frac{\partial^{2}}{\partial d_{1} \partial d_{2}}+\frac{\partial^{2}}{\partial a_{2} \partial d_{1}}
\end{array}\right) a_{1}^{l-m} a_{2}^{-l+m-1} d_{1}^{l+m} d_{2}^{-l-m-1} \\
& \quad=24\left(\begin{array}{cc}
\frac{\partial^{2}}{\partial a_{1} \partial a_{2}}+\frac{\partial^{2}}{\partial a_{1} \partial d_{2}} & 0 \\
0 & \frac{\partial^{2}}{\partial d_{1} \partial d_{2}}+\frac{\partial^{2}}{\partial a_{2} \partial d_{1}}
\end{array}\right) \frac{\log \left(1-d_{1} / d_{2}\right)-\log \left(1-a_{1} / a_{2}\right)}{a_{2} d_{2}\left(a_{1} / a_{2}-d_{1} / d_{2}\right)} .
\end{aligned}
$$

Now suppose that $Z_{1} \in \mathbb{D}_{R}^{-}$and $Z_{2} \in \mathbb{D}_{R}^{+}$, then

$$
\begin{aligned}
& \left(J_{R} N(W)^{-1}\right)\left(Z_{1}, Z_{2}\right)=24 \vec{\partial}_{Z_{1}}\left(\left(I_{R} N(W)^{-1}\right)\left(Z_{1}, Z_{2}\right)\right) \overleftarrow{\partial}_{Z_{2}} \\
& =-\sum_{l, m, n} \frac{24}{2 l+1} \frac{1}{N\left(Z_{1}\right)}\left(\begin{array}{ll}
(l-m+1) t_{m-\frac{1}{2} n-\frac{1}{2}}^{l+\frac{1}{2}}\left(Z_{1}^{-1}\right) & (l-m+1) t_{m-\frac{1}{2} \frac{n+\frac{1}{2}}{2}}^{l+\frac{1}{2}}\left(Z_{1}^{-1}\right) \\
(l+m+1) t_{m+\frac{1}{2} \underline{n-\frac{1}{2}}}^{l+\frac{1}{2}}\left(Z_{1}^{-1}\right) & (l+m+1) t_{m+\frac{1}{2} \underline{n+\frac{1}{2}}}^{l+\frac{1}{2}}\left(Z_{1}^{-1}\right)
\end{array}\right)
\end{aligned}
$$

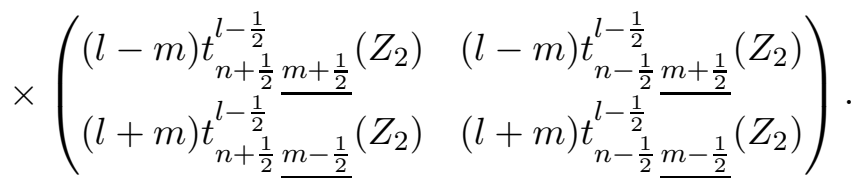

If $Z_{1}$ and $Z_{2}$ are diagonal: $Z_{1}=\left(\begin{array}{cc}a_{1} & 0 \\ 0 & d_{1}\end{array}\right)$ with $\left|a_{1}\right|,\left|d_{1}\right|>R$ and $Z_{2}=\left(\begin{array}{cc}a_{2} & 0 \\ 0 & d_{2}\end{array}\right)$ with $\left|a_{2}\right|,\left|d_{2}\right|<R$, then, by (64), only the terms with $n=m-1, m, m+1$ are non-zero, and it is easy to see that

$$
\begin{aligned}
& \left(J_{R} N(W)^{-1}\right)\left(Z_{1}, Z_{2}\right) \\
& =\sum_{l, m} \frac{24}{2 l+1}\left(\begin{array}{cc}
\frac{\partial^{2}}{\partial a_{1} \partial a_{2}}+\frac{\partial^{2}}{\partial a_{1} \partial d_{2}} & 0 \\
0 & \frac{\partial^{2}}{\partial d_{1} \partial d_{2}}+\frac{\partial^{2}}{\partial a_{2} \partial d_{1}}
\end{array}\right) a_{1}^{-l+m-1} a_{2}^{l-m} d_{1}^{-l-m-1} d_{2}^{l+m} \\
& \quad=24\left(\begin{array}{cc}
\frac{\partial^{2}}{\partial a_{1} \partial a_{2}}+\frac{\partial^{2}}{\partial a_{1} \partial d_{2}} & 0 \\
0 & \frac{\partial^{2}}{\partial d_{1} \partial d_{2}}+\frac{\partial^{2}}{\partial a_{2} \partial d_{1}}
\end{array}\right) \frac{\log \left(1-d_{2} / d_{1}\right)-\log \left(1-a_{2} / a_{1}\right)}{a_{1} d_{1}\left(a_{2} / a_{1}-d_{2} / d_{1}\right)}
\end{aligned}
$$

Adding (65) and (66), we obtain

$$
\begin{aligned}
\left(\left(J_{R}^{+-}+J_{R}^{-+}\right) N(W)^{-1}\right)\left(Z_{1}, Z_{2}\right) & \\
& =24\left(\begin{array}{cc}
\frac{\partial^{2}}{\partial a_{1} \partial a_{2}}+\frac{\partial^{2}}{\partial a_{1} \partial d_{2}} & 0 \\
0 & \frac{\partial^{2}}{\partial d_{1} \partial d_{2}}+\frac{\partial^{2}}{\partial a_{2} \partial d_{1}}
\end{array}\right) \frac{\log \left(a_{1} / a_{2}\right)-\log \left(d_{1} / d_{2}\right)}{a_{2} d_{2}\left(a_{1} / a_{2}-d_{1} / d_{2}\right)}
\end{aligned}
$$

where $Z_{1}=\left(\begin{array}{cc}a_{1} & 0 \\ 0 & d_{1}\end{array}\right), Z_{2}=\left(\begin{array}{cc}a_{2} & 0 \\ 0 & d_{2}\end{array}\right) \in U(2)_{R}$. Note that in this equation log denotes the branch of logarithm with a cut along the positive real axis. Finally, we take a limit of (67) as $Z_{1}, Z_{2} \rightarrow$ $Z=\left(\begin{array}{ll}a & 0 \\ 0 & d\end{array}\right) \in U(2)_{R}$. Since we know that the limit exists, to find its value, we can, for example, set $a_{1}=a, d_{1}=d_{2}=d$ and let $a_{1} / a_{2} \rightarrow 1$. We obtain

$$
\left(\left(J_{R}^{+-}+J_{R}^{-+}\right) N(W)^{-1}\right)\left(Z_{1}, Z_{2}\right) \rightarrow-\frac{4}{a^{2} d^{2}}\left(\begin{array}{cc}
1+2 d / a & 0 \\
0 & 1+2 a / d
\end{array}\right)=-\operatorname{Mx}\left(\frac{1}{N(Z)}\right)
$$




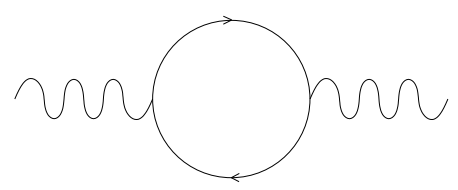

(a) Spinor case

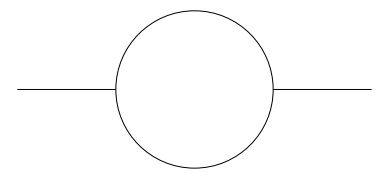

(b) Scalar case

Figure 8: Vacuum polarization Feynman diagrams.

as $Z_{1}, Z_{2} \rightarrow Z=\left(\begin{array}{ll}a & 0 \\ 0 & d\end{array}\right) \in U(2)_{R}$ (recall our earlier computation (63)). This completes our proof that $\mathrm{Mx}^{0} N(Z)^{-1}=\mathrm{Mx} N(Z)^{-1}$.

We note that Theorem 67 in this paper and Theorem 15 in [FL3] can be viewed as mathematical versions of the regularization of vacuum polarization in QED and scalar QED, as it was discussed in Subsection 4.5 of [FL1]. One just has to add, respectively, operators $J_{R}^{++}+J_{R}^{--}$ and $I_{R}^{++}+I_{R}^{--}$, which do not contain any singularities and are also related by the same identity (61). Note, however, that the non-scalar case (Theorem 67) contains an additional subtraction of the one-dimensional representation component from $F \in \mathcal{W}^{\prime}$ (after factorization by the intersection of the kernels of the maps $J_{R}^{ \pm \pm}$). Thus, one can say that the trivial one-dimensional component of $\mathcal{W}^{\prime}$ that appears in the decomposition of $\left(\rho_{2}^{\prime}, \mathcal{W}^{\prime}\right)$ into irreducible components in Subsection 5.2 lies at the heart of the regularization of the vacuum polarization. The subtraction of this component in the Minkowski picture is a subtle procedure that is a part of the art of renormalization in four-dimensional QED.

\section{Algebras of Quaternionic Functions}

In this section we construct $\mathfrak{g l}\left(2, \mathbb{H}_{\mathbb{C}}\right)$-invariant algebra structures on $\left(\rho_{1}, \mathscr{T}\right)$ and $\left(\rho_{2}^{\prime}, \mathcal{W}^{\prime} /\right.$ ker $\left.\mathrm{Mx}\right)$. The first two subsections deal with the scalar case $\left(\rho_{1}, \mathscr{W}\right)$ and the following two subsections deal with the spinor case $\left(\rho_{2}^{\prime}, \mathcal{W}^{\prime} /\right.$ ker $\left.\mathrm{Mx}\right)$. In the last subsection we conjecture that these algebras have the structures of weak cyclic $A_{\infty}$-algebras.

\subsection{Scalar Version of the Convolution Algebra}

Recall harmonic polynomial functions on $\mathbb{H}_{\mathbb{C}}^{\times}$:

$$
\begin{aligned}
\mathcal{H}^{+} & =\left\{\varphi \in \mathbb{C}\left[z_{11}, z_{12}, z_{21}, z_{22}\right] ; \square \varphi=0\right\} \\
& =\mathbb{C} \text {-span of }\left\{t_{n \underline{m}}^{l}(Z)\right\}, \\
\mathcal{H}^{-} & =\left\{\varphi \in \mathbb{C}\left[z_{11}, z_{12}, z_{21}, z_{22}, N(Z)^{-1}\right] ; N(Z)^{-1} \cdot \varphi\left(Z^{-1}\right) \in \mathcal{H}^{+}\right\} \\
& =\mathbb{C} \text {-span of }\left\{N(Z)^{-1} \cdot t_{m \underline{n}}^{l}\left(Z^{-1}\right)\right\}, \\
\mathcal{H} & =\left\{\varphi \in \mathbb{C}\left[z_{11}, z_{12}, z_{21}, z_{22}, N(Z)^{-1}\right] ; \square \varphi=0\right\} \\
& =\mathcal{H}^{+} \oplus \mathcal{H}^{-},
\end{aligned}
$$

where $l=0, \frac{1}{2}, 1, \frac{3}{2}, \ldots, m, n \in \mathbb{Z}+l,-l \leq m, n \leq l$. The Lie algebra $\mathfrak{g l}\left(2, \mathbb{H}_{\mathbb{C}}\right)$ acts on $\mathcal{H}^{+}$, $\mathcal{H}^{-}$and $\mathcal{H}$ via two slightly different actions $\pi_{l}^{0}$ and $\pi_{r}^{0}$ (Subsections 2.4-2.5 in [FL1]). There is a non-degenerate $\mathfrak{g l}\left(2, \mathbb{H}_{\mathbb{C}}\right)$-invariant bilinear pairing between $\left(\pi_{l}^{0}, \mathcal{H}^{+}\right)$and $\left(\pi_{r}^{0}, \mathcal{H}^{-}\right)$given by the integral formula

$$
\left\langle\varphi_{1}, \varphi_{2}\right\rangle_{\mathcal{H}}=\frac{1}{2 \pi^{2}} \int_{Z \in S U(2)}\left(\widetilde{\operatorname{deg}}_{Z} \varphi_{1}\right)(Z) \cdot \varphi_{2}(Z) d S, \quad \varphi_{1} \in \mathcal{H}^{+}, \varphi_{2} \in \mathcal{H}^{-},
$$


(equation (32) in [FL1]). We extend this pairing to $\mathcal{H}^{-} \times \mathcal{H}^{+}$by the same formula (or antisymmetry), and then declare pairings on $\mathcal{H}^{+} \times \mathcal{H}^{+}$and $\mathcal{H}^{-} \times \mathcal{H}^{-}$to be zero (even though the integral need not be zero in these cases). Thus we obtain a non-degenerate antisymmetric $\mathfrak{g l}\left(2, \mathbb{H}_{\mathbb{C}}\right)$-invariant bilinear pairing between $\left(\pi_{l}^{0}, \mathcal{H}\right)$ and $\left(\pi_{r}^{0}, \mathcal{H}\right)$.

We consider $\mathcal{H} \otimes \mathcal{H}$. This space consists of polynomial $\mathbb{C}$-valued functions in two variables $Z_{1}, Z_{2} \in \mathbb{H}_{\mathbb{C}}^{\times}$that are harmonic with respect to $Z_{1}$ and $Z_{2}$. We define a convolution operation on $\mathcal{H} \otimes \mathcal{H}$ as

$$
(f * g)\left(Z_{1}, Z_{2}\right)=\left\langle\varphi_{2}, \varphi_{1}^{\prime}\right\rangle_{\mathcal{H}} \cdot \varphi_{1}\left(Z_{1}\right) \otimes \varphi_{2}^{\prime}\left(Z_{2}\right)
$$

where $f=\varphi_{1}\left(Z_{1}\right) \otimes \varphi_{2}\left(Z_{2}\right), g=\varphi_{1}^{\prime}\left(Z_{1}\right) \otimes \varphi_{2}^{\prime}\left(Z_{2}\right) \in \mathcal{H} \otimes \mathcal{H}$. This operation gives $\mathcal{H} \otimes \mathcal{H}$ the structure of an associative algebra. Since the bilinear pairing is $\mathfrak{g l}\left(2, \mathbb{H}_{\mathbb{C}}\right)$-invariant, the above convolution product is $\mathfrak{g l}\left(2, \mathbb{H}_{\mathbb{C}}\right)$-equivariant with respect to the $\pi_{l}^{0} \otimes \pi_{r}^{0}$ action on $\mathcal{H} \otimes \mathcal{H}$.

Next, we consider a space $\mathcal{H} \mathcal{H}$ consisting of infinite sums $\sum_{i} \varphi_{i}\left(Z_{1}\right) \otimes \varphi_{i}^{\prime}\left(Z_{2}\right)$, where $\varphi_{i}, \varphi_{i}^{\prime} \in$ $\mathcal{H}$, such that

$$
\mid \text { degree of } \varphi_{i}+\text { degree of } \varphi_{i}^{\prime} \mid
$$

is bounded. More precisely, $\mathcal{H} \mathcal{H}$ consists of formal series of the form

$$
\begin{aligned}
\sum_{\substack{l, m, n \\
l^{\prime}, m^{\prime}, n^{\prime}}} a_{\substack{l, m, n, n \\
l^{\prime}, m^{\prime}, n^{\prime}}} t_{n \underline{m}}^{l}\left(Z_{1}\right) \cdot t_{n^{\prime} \underline{m^{\prime}}}^{l^{\prime}}\left(Z_{2}\right)+\sum_{\substack{l, m, n \\
l^{\prime}, m^{\prime}, n^{\prime}}} b_{\substack{l, m, n \\
l^{\prime}, m^{\prime}, n^{\prime}}} t_{n \underline{m}}^{l}\left(Z_{1}\right) \cdot N\left(Z_{2}\right)^{-1} \cdot t_{m^{\prime} \underline{n^{\prime}}}^{l^{\prime}}\left(Z_{2}^{-1}\right) \\
+\sum_{\substack{l, m, n \\
l^{\prime}, m^{\prime}, n^{\prime}}} c_{\substack{l, l, m, n \\
l^{\prime}, m^{\prime}, n^{\prime}}} N\left(Z_{1}\right)^{-1} \cdot t_{m \underline{n}}^{l}\left(Z_{1}^{-1}\right) \cdot t_{n^{\prime} \underline{m^{\prime}}}^{l^{\prime}}\left(Z_{2}\right) \\
+\sum_{\substack{l, m, n \\
l^{\prime}, m^{\prime}, n^{\prime}}} d_{\substack{l, m, n, n \\
l^{\prime}, m^{\prime}, n^{\prime}}} N\left(Z_{1}\right)^{-1} \cdot t_{m \underline{n}}^{l}\left(Z_{1}^{-1}\right) \cdot N\left(Z_{2}\right)^{-1} \cdot t_{m^{\prime} \underline{n^{\prime}}}^{l^{\prime}}\left(Z_{2}^{-1}\right)
\end{aligned}
$$

such that only finitely many coefficients $a_{\substack{l, m, n \\ l^{\prime}, m^{\prime}, n^{\prime}}}$ 's, $d_{l^{\prime}, m^{\prime}, n^{\prime}}^{l, m, n}$, $\mathrm{s}$ are non-zero and non-zero coefficients $b_{l^{\prime}, m^{\prime}, n^{\prime}}^{l, m, n}$ 's, $c_{l^{\prime}, m^{\prime}, n^{\prime}}^{l, m, n}$ 's have bounded difference of indices $\left|l-l^{\prime}\right|$.

Lemma 68. The convolution operation (68) extends to $\mathcal{H} \mathcal{H}$ and gives it the structure of a $\mathfrak{g l}\left(2, \mathbb{H}_{\mathbb{C}}\right)$-invariant associative algebra. Moreover, if $f \in \mathcal{H} \otimes \mathcal{H}$ and $g \in \mathcal{H} \mathcal{H}$, then both $f * g$ and $g * f$ lie in $\mathcal{H} \otimes \mathcal{H}$.

Proof. The result follows from an observation that, for a fixed index $l_{0}$, there are only finitely many non-zero coefficients

$$
\underset{l^{\prime}, m^{\prime}, n^{\prime}}{a}, \quad b_{l^{\prime}, m^{\prime}, n^{\prime}}, \quad c_{l^{\prime}, m^{\prime}, n^{\prime}}^{l_{0}, m, n}, \quad d_{l^{\prime}, m^{\prime}, n^{\prime}}
$$

with that particular index, and similarly for index $l_{0}^{\prime}$.

Unlike $\mathcal{H} \otimes \mathcal{H}, \mathcal{H} \mathcal{H}$ has a unit. The expression for the unit is obtained by formally combining two copies of matrix coefficient expansions of $N\left(Z_{1}-Z_{2}\right)^{-1}$ given in Proposition 25 from [FL1] (see also Proposition 112):

$$
1_{\mathcal{H} \mathcal{H}}=-\sum_{l, m, n} t_{n \underline{m}}^{l}\left(Z_{1}\right) \cdot N\left(Z_{2}\right)^{-1} \cdot t_{m \underline{n}}^{l}\left(Z_{2}^{-1}\right)+\sum_{l, m, n} N\left(Z_{1}\right)^{-1} \cdot t_{m \underline{n}}^{l}\left(Z_{1}^{-1}\right) \cdot t_{n \underline{m}}^{l}\left(Z_{2}\right) \quad \in \mathcal{H} \mathcal{H}
$$

The fact that $1_{\mathcal{H H}}$ is indeed a unit follows from the definition of the convolution operation and orthogonality relations (17) in [FL3].

The Lie algebra $\mathfrak{g l}\left(2, \mathbb{H}_{\mathbb{C}}\right)$ can act on $\mathcal{H} \otimes \mathcal{H}$ by at least three different actions: $\pi_{l}^{0} \otimes 1,1 \otimes \pi_{r}^{0}$ and $\pi_{l}^{0} \otimes \pi_{r}^{0}$. Clearly, all three actions extend to $\mathcal{H} \mathcal{H}$. Then the convolution operation on $\mathcal{H} \mathcal{H}$ is $\left(\pi_{l}^{0} \otimes \pi_{r}^{0}\right)$-equivariant. 
For each $d \in \mathbb{Z}$, we define operators $(\operatorname{deg}+d)_{Z_{1}}^{-1}$ and $(\operatorname{deg}+d)_{Z_{2}}^{-1}$ on $\mathcal{H} \mathcal{H}$ as follows. If $f \in \mathcal{H} \mathcal{H}$ is expressed as a series (69), then

$$
\begin{aligned}
& (\operatorname{deg}+d)_{Z_{1}}^{-1} f\left(Z_{1}, Z_{2}\right)=\sum_{\substack{l>-d / 2 \\
m, n, l^{\prime}, m^{\prime}, n^{\prime}}} \frac{a_{l^{l, m, m^{\prime}, n^{\prime}}}^{l, n}}{2 l+d} t_{n \underline{m}}^{l}\left(Z_{1}\right) \cdot t_{n^{\prime} \underline{m^{\prime}}}^{l^{\prime}}\left(Z_{2}\right)
\end{aligned}
$$

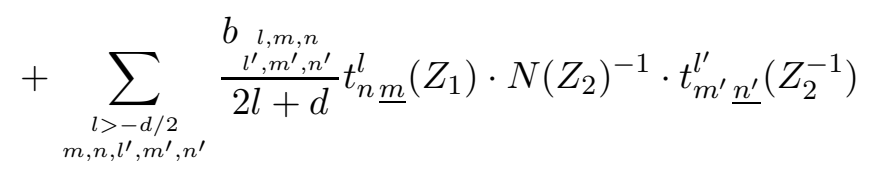

$$
\begin{aligned}
& -\sum_{\substack{l>d / 2-1 \\
m, n, l^{\prime}, m^{\prime}, n^{\prime}}} \frac{c_{\substack{l, m, n \\
l^{\prime}, m^{\prime}, n^{\prime}}}}{2 l+2-d} N\left(Z_{1}\right)^{-1} \cdot t_{m \underline{n}}^{l}\left(Z_{1}^{-1}\right) \cdot t_{n^{\prime}}^{l^{\prime}}{\underline{m^{\prime}}}^{\prime}\left(Z_{2}\right) \\
& -\sum_{\substack{l>d / 2-1 \\
m, n, l^{\prime}, m^{\prime}, n^{\prime}}} \frac{d_{\substack{l, m, n \\
l^{\prime}, m^{\prime}, n^{\prime}}}}{2 l+2-d} N\left(Z_{1}\right)^{-1} \cdot t_{m \underline{n}}^{l}\left(Z_{1}^{-1}\right) \cdot N\left(Z_{2}\right)^{-1} \cdot t_{m^{\prime} \underline{\prime}^{\prime}}^{l^{\prime}}\left(Z_{2}^{-1}\right) .
\end{aligned}
$$

Note that if $d \neq 1$, certain terms get discarded: if $d>1$, terms

$$
N\left(Z_{1}\right)^{-1} \cdot t_{m \underline{n}}^{l}\left(Z_{1}^{-1}\right) \cdot t_{n^{\prime} \underline{m^{\prime}}}^{l^{\prime}}\left(Z_{2}\right) \quad \text { and } \quad N\left(Z_{1}\right)^{-1} \cdot t_{m \underline{n}}^{l}\left(Z_{1}^{-1}\right) \cdot N\left(Z_{2}\right)^{-1} \cdot t_{m^{\prime}{\underline{n^{\prime}}}^{\prime}}^{l^{\prime}}\left(Z_{2}^{-1}\right)
$$

with $0 \leq l \leq d / 2-1$ are discarded; and if $d<1$, terms

$$
t_{n \underline{m}}^{l}\left(Z_{1}\right) \cdot t_{n^{\prime} \underline{m^{\prime}}}^{l^{\prime}}\left(Z_{2}\right) \quad \text { and } \quad t_{n \underline{m}}^{l}\left(Z_{1}\right) \cdot N\left(Z_{2}\right)^{-1} \cdot t_{m^{\prime} \underline{n^{\prime}}}^{l^{\prime}}\left(Z_{2}^{-1}\right)
$$

with $0 \leq l \leq-d / 2$ are discarded. Then $(\operatorname{deg}+d)_{Z_{2}}^{-1} f\left(Z_{1}, Z_{2}\right)$ is defined similarly.

Lemma 69. Let $f\left(Z_{1}, Z_{2}\right) \in \mathcal{H} \mathcal{H}, d \in \mathbb{Z}$ and $A, B, C, D \in \mathbb{H}_{\mathbb{C}}$. We have the following commutation relations:

$$
\begin{aligned}
& (\operatorname{deg}+d)_{Z_{1}}^{-1}\left(\pi_{l}^{0} \otimes 1\right)\left(\begin{array}{cc}
A & 0 \\
0 & D
\end{array}\right) f\left(Z_{1}, Z_{2}\right)=\left(\pi_{l}^{0} \otimes 1\right)\left(\begin{array}{cc}
A & 0 \\
0 & D
\end{array}\right)(\operatorname{deg}+d)_{Z_{1}}^{-1} f\left(Z_{1}, Z_{2}\right), \\
& (\operatorname{deg}+d)_{Z_{1}}^{-1}\left(\pi_{l}^{0} \otimes 1\right)\left(\begin{array}{cc}
0 & B \\
0 & 0
\end{array}\right) f\left(Z_{1}, Z_{2}\right)=\left(\pi_{l}^{0} \otimes 1\right)\left(\begin{array}{cc}
0 & B \\
0 & 0
\end{array}\right)(\operatorname{deg}+d-1)_{Z_{1}}^{-1} f\left(Z_{1}, Z_{2}\right), \\
& (\operatorname{deg}+d)_{Z_{1}}^{-1}\left(\pi_{l}^{0} \otimes 1\right)\left(\begin{array}{cc}
0 & 0 \\
C & 0
\end{array}\right) f\left(Z_{1}, Z_{2}\right)=\left(\pi_{l}^{0} \otimes 1\right)\left(\begin{array}{cc}
0 & 0 \\
C & 0
\end{array}\right)(\operatorname{deg}+d+1)_{Z_{1}}^{-1} f\left(Z_{1}, Z_{2}\right),
\end{aligned}
$$

and similarly for $(\operatorname{deg}+d)_{Z_{2}}^{-1}$ and $1 \otimes \pi_{r}^{0}$.

Lemma 70. Let $f, g \in \mathcal{H} \mathcal{H}$ and $d \in \mathbb{Z}$. We have the following relations:

$$
\begin{aligned}
& (\operatorname{deg}+d)_{Z_{1}}^{-1}(f * g)=\left((\operatorname{deg}+d)_{Z_{1}}^{-1} f\right) * g, \\
& (\operatorname{deg}+d)_{Z_{2}}^{-1}(f * g)=f *\left((\operatorname{deg}+d)_{Z_{2}}^{-1} g\right), \\
& \left((\operatorname{deg}+d)_{Z_{2}}^{-1} f\right) * g=-f *\left((\operatorname{deg}+2-d)_{Z_{1}}^{-1} g\right) .
\end{aligned}
$$

We define an equivariant map

$$
I=I^{++}-\left(I^{+-}+I^{-+}\right)+I^{--}:\left(\rho_{1}, \mathcal{W}\right) \rightarrow\left(\pi_{l}^{0} \otimes \pi_{r}^{0}, \mathcal{H} \mathcal{H}\right)
$$

as follows. Recall the maps $I_{R}$ given by equation (34) in Chapter 6 of [FL3, their definition is copied here in (58). By Lemma 11 and Theorem 12 in [FL3], if $Z_{1}, Z_{2} \in \mathbb{D}_{R}^{+}$, $I_{R}$ is a $\mathfrak{g l}\left(2, \mathbb{H}_{\mathbb{C}}\right)$-equivariant map $\left(\rho_{1}, \mathscr{K}\right) \rightarrow\left(\pi_{l}^{0} \otimes \pi_{r}^{0}, \mathcal{H}^{+} \otimes \mathcal{H}^{+}\right)$independent of the choice of $R>0$; 
we call this map $I^{++}$. Similarly, if $Z_{1}, Z_{2} \in \mathbb{D}_{R}^{-}, I_{R}$ is a $\mathfrak{g l}\left(2, \mathbb{H}_{\mathbb{C}}\right)$-equivariant map $\left(\rho_{1}, \mathscr{T}\right) \rightarrow$ $\left(\pi_{l}^{0} \otimes \pi_{r}^{0}, \mathcal{H}^{-} \otimes \mathcal{H}^{-}\right)$also independent of the choice of $R>0$; we call this map $I^{--}$. If $f \in \mathcal{W}$,

$$
I^{+-}(f)=\sum_{\substack{l, m, n \\ l^{\prime}, m^{\prime}, n^{\prime}}} b(f)_{\substack{l, m, n \\ l^{\prime}, m^{\prime}, n^{\prime}}} t_{n \underline{m}}^{l}\left(Z_{1}\right) \cdot N\left(Z_{2}\right)^{-1} \cdot t_{m^{\prime} \underline{n^{\prime}}}^{l^{\prime}}\left(Z_{2}^{-1}\right)
$$

where

$$
b(f)_{\substack{l, m, m, n \\ l^{\prime}, m^{\prime}, n^{\prime}}}^{l,}=\frac{i}{2 \pi^{3}} \int_{U(2)_{R}} f(W) \cdot N(W)^{-1} \cdot t_{m \underline{n}}^{l}\left(W^{-1}\right) \cdot t_{n^{\prime} \underline{m}^{\prime}}^{l^{\prime}}(W) d V .
$$

On the one hand, this integral does not depend on $R>0$. On the other hand, by the matrix coefficient expansion of $N(Z-W)^{-1}$ given in Proposition 25 from [FL1] (see also Proposition 112), for each $R>0$, the series $I^{+-}(f)$ converges to $\left(I_{R} f\right)\left(Z_{1}, Z_{2}\right)$ whenever $Z_{1} \in \mathbb{D}_{R}^{+}$and $Z_{2} \in \mathbb{D}_{R}^{-}$. Similarly,

$$
I^{-+}(f)=\sum_{\substack{l, m, n \\ l^{\prime}, m^{\prime}, n^{\prime}}} c(f) \underset{\substack{l, m, n \\ l^{\prime}, m^{\prime}, n^{\prime}}}{l} N\left(Z_{1}\right)^{-1} \cdot t_{m \underline{n}}^{l}\left(Z_{1}^{-1}\right) \cdot t_{n^{\prime}}^{l^{\prime}} \underline{m^{\prime}}\left(Z_{2}\right)
$$

where

$$
c(f)_{\substack{l, m, n \\ l^{\prime}, m^{\prime}, n^{\prime}}}=b(f)_{\substack{l^{\prime}, m^{\prime}, n^{\prime} \\ l, m, n}}=\frac{i}{2 \pi^{3}} \int_{U(2)_{R}} f(W) \cdot t_{n \underline{m}}^{l}(W) \cdot N(W)^{-1} \cdot t_{m^{\prime}{\underline{n^{\prime}}}^{l^{\prime}}}^{{ }_{l}}\left(W^{-1}\right) d V .
$$

This integral is independent of $R>0$ and, for each $R>0$, the series $I^{-+}(f)$ converges to $\left(I_{R} f\right)\left(Z_{1}, Z_{2}\right)$ whenever $Z_{1} \in \mathbb{D}_{R}^{-}$and $Z_{2} \in \mathbb{D}_{R}^{+}$.

Proposition 71. We have:

$$
I\left(N(Z)^{-1}\right)=(\operatorname{deg}+1)_{Z_{1}}^{-1} 1_{\mathcal{H} H}=-(\operatorname{deg}+1)_{Z_{2}}^{-1} 1_{\mathcal{H} H} .
$$

In particular, for any $g \in \mathcal{H} \mathcal{H}$,

$$
I\left(N(Z)^{-1}\right) * g=(\operatorname{deg}+1)_{Z_{1}}^{-1} g, \quad g * I\left(N(Z)^{-1}\right)=-(\operatorname{deg}+1)_{Z_{2}}^{-1} g .
$$

Proof. From Section 6 in [FL3], we have:

$$
\begin{aligned}
& I\left(N(Z)^{-1}\right)\left(Z_{1}, Z_{2}\right) \\
& \quad=-\sum_{l, m, n} \frac{N\left(Z_{2}\right)^{-1}}{2 l+1} t_{n \underline{m}}^{l}\left(Z_{1}\right) \cdot t_{m \underline{n}}^{l}\left(Z_{2}^{-1}\right)-\sum_{l, m, n} \frac{N\left(Z_{1}\right)^{-1}}{2 l+1} t_{m \underline{n}}^{l}\left(Z_{1}^{-1}\right) \cdot t_{n \underline{m}}^{l}\left(Z_{2}\right) .
\end{aligned}
$$

Then the result follows from (70) and Lemma 70 .

\subsection{Scalar Version of the Algebra of Quaternionic Functions}

In this subsection we give $\left(\rho_{1}, \mathscr{W}\right)$ the structure of a $\mathfrak{g l}\left(2, \mathbb{H}_{\mathbb{C}}\right)$-invariant algebra.

Definition 72. Let $\mathcal{H} \mathcal{H}^{\omega}$ denote the subspace of $\mathcal{H} \mathcal{H}$ generated by $\mathcal{H} \otimes \mathcal{H}, I(\mathscr{W})$, application of operators $(\operatorname{deg}+d)_{Z_{1}}^{-1}$ and $(\operatorname{deg}+d)_{Z_{2}}^{-1}, d \in \mathbb{Z}$, as well as actions $\pi_{l}^{0} \otimes 1$ and $1 \otimes \pi_{r}^{0}$ of $\mathfrak{g l}\left(2, \mathbb{H}_{\mathbb{C}}\right)$.

Thus, by definition, $\mathcal{H H}^{\omega}$ is invariant under the $\pi_{l}^{0} \otimes \pi_{r}^{0}$ action of $\mathfrak{g l}\left(2, \mathbb{H}_{\mathbb{C}}\right)$. We want to reduce the number of generators of $\mathcal{H H}^{\omega}$. 
Lemma 73. The space $\mathcal{H} \mathcal{H}^{\omega}$ is generated by $\mathcal{H} \otimes \mathcal{H}$, elements of the type

$$
\begin{gathered}
I\left(N(Z)^{-1}\right), \quad\left(\operatorname{deg}+d_{1}\right)_{Z_{1}}^{-1} I\left(N(Z)^{-1}\right), \quad\left(\operatorname{deg}+d_{1}\right)_{Z_{2}}^{-1} I\left(N(Z)^{-1}\right), \\
\left(\operatorname{deg}+d_{2}\right)_{Z_{1}}^{-1}\left(\operatorname{deg}+d_{1}\right)_{Z_{1}}^{-1} I\left(N(Z)^{-1}\right), \quad\left(\operatorname{deg}+d_{2}\right)_{Z_{1}}^{-1}\left(\operatorname{deg}+d_{1}\right)_{Z_{2}}^{-1} I\left(N(Z)^{-1}\right), \\
\left(\operatorname{deg}+d_{2}\right)_{Z_{2}}^{-1}\left(\operatorname{deg}+d_{1}\right)_{Z_{2}}^{-1} I\left(N(Z)^{-1}\right), \quad\left(\operatorname{deg}+d_{3}\right)_{Z_{1}}^{-1}\left(\operatorname{deg}+d_{2}\right)_{Z_{1}}^{-1}\left(\operatorname{deg}+d_{1}\right)_{Z_{1}}^{-1} I\left(N(Z)^{-1}\right), \ldots
\end{gathered}
$$

as well as actions $\pi_{l}^{0} \otimes 1$ and $1 \otimes \pi_{r}^{0}$ of $\mathfrak{g l}\left(2, \mathbb{H}_{\mathbb{C}}\right)$.

Proof. Since $\mathscr{K}=\mathscr{K}^{-} \oplus \mathscr{K}^{0} \oplus \mathscr{K}^{+}, I\left(\mathscr{K}^{-} \oplus \mathscr{K}^{+}\right) \subset \mathcal{H} \otimes \mathcal{H}$ and $\mathscr{K}^{0}$ is generated by $N(Z)^{-1}$, $\mathcal{H} \mathcal{H}^{\omega}$ can be generated by $\mathcal{H} \otimes \mathcal{H}$ and $I\left(N(Z)^{-1}\right)$ instead of $\mathcal{H} \otimes \mathcal{H}$ and $I(\mathscr{W})$. Notice that applying $(\operatorname{deg}+d)_{Z_{1}}^{-1}$ or $(\operatorname{deg}+d)_{Z_{2}}^{-1}$ to an element of $\mathcal{H} \otimes \mathcal{H}$ results in another element of $\mathcal{H} \otimes \mathcal{H}$. Then the result follows from Lemma 69.

Proposition 74. The space $\mathcal{H H}^{\omega}$ is closed under the convolution operation: if $f, g \in \mathcal{H H}^{\omega}$, then $f * g$ also lies in $\mathcal{H H}^{\omega}$.

Proof. First, observe that if $f$ or $g \in \mathcal{H} \otimes \mathcal{H}$, then, by Lemma 68,

$$
f * g \in \mathcal{H} \otimes \mathcal{H} \subset \mathcal{H} \mathcal{H}^{\omega} .
$$

Since the convolution operation is $\left(\pi_{l}^{0} \otimes \pi_{r}^{0}\right)$-equivariant,

$$
\left(\left(\pi_{l}^{0} \otimes \pi_{r}^{0}\right)(X) f\right) * g=\left(\pi_{l}^{0} \otimes \pi_{r}^{0}\right)(X)(f * g)-f *\left(\left(\pi_{l}^{0} \otimes \pi_{r}^{0}\right)(X) g\right),
$$

for all $X \in \mathfrak{g l}\left(2, \mathbb{H}_{\mathbb{C}}\right)$. And by Lemmas 70 and 73 , it is sufficient to prove the proposition for the case $f$ is one of the generators of $\mathcal{H} \mathcal{H}^{\omega}$ of the form $I\left(N(Z)^{-1}\right)$ with operators $\left(\operatorname{deg}+d_{i}\right)_{Z_{1}}^{-1}$ and $\left(\operatorname{deg}+d_{j}\right)_{Z_{2}}^{-1}$ applied several times. Then Lemma 70 reduces this further to the case $f=$ $I\left(N(Z)^{-1}\right)$, and the result follows from Proposition 71 .

Next, we realize elements of $\mathcal{H} \mathcal{H}^{\omega}$ as analytic functions as opposed to formal series. For $Z_{1}, Z_{2} \in \mathbb{H}_{\mathbb{C}}^{\times}$, let $\lambda_{1}$ and $\lambda_{2}$ denote the eigenvalues of $Z_{1} Z_{2}^{-1}$, and introduce open subsets of $\mathbb{H}_{\mathbb{C}}^{\times} \times \mathbb{H}_{\mathbb{C}}^{\times}$

$$
\begin{aligned}
\Omega & =\left\{\left(Z_{1}, Z_{2}\right) \in \mathbb{H}_{\mathbb{C}}^{\times} \times \mathbb{H}_{\mathbb{C}}^{\times} ; \text {neither } \lambda_{1} \text { nor } \lambda_{2} \text { is a positive real number }\right\}, \\
\Lambda^{+} & =\left\{\left(Z_{1}, Z_{2}\right) \in \mathbb{H}_{\mathbb{C}}^{\times} \times \mathbb{H}_{\mathbb{C}}^{\times} ; \operatorname{sign}\left(\operatorname{Im} \lambda_{1}\right)>0, \operatorname{sign}\left(\operatorname{Im} \lambda_{2}\right)>0\right\}, \\
\Lambda^{-} & =\left\{\left(Z_{1}, Z_{2}\right) \in \mathbb{H}_{\mathbb{C}}^{\times} \times \mathbb{H}_{\mathbb{C}}^{\times} ; \operatorname{sign}\left(\operatorname{Im} \lambda_{1}\right)<0, \operatorname{sign}\left(\operatorname{Im} \lambda_{2}\right)<0\right\} .
\end{aligned}
$$

Then $\Lambda^{+}, \Lambda^{-} \subset \Omega$.

Lemma 75. The elements of $\mathcal{H} \mathcal{H}^{\omega}$ can be realized as analytic functions on $\Omega$.

Proof. Elements of $\mathcal{H} \otimes \mathcal{H}$ are polynomials, hence can be treated as analytic functions on $\Omega$. On the other hand,

$$
I\left(N(Z)^{-1}\right)=-I^{+-}\left(N(Z)^{-1}\right)-I^{-+}\left(N(Z)^{-1}\right) .
$$

As was shown in Section 6 of [FL3], the series $I^{+-}\left(N(Z)^{-1}\right)$ converges on $\mathbb{D}_{R}^{+} \times \mathbb{D}_{R}^{-}$and extends analytically to $\Omega$. Similarly, the series $I^{-+}\left(N(Z)^{-1}\right)$ converges on $\mathbb{D}_{R}^{-} \times \mathbb{D}_{R}^{+}$and also extends analytically to $\Omega$. Just as we expressed $(\operatorname{deg}+2)^{-1}$ as an integral operator in Subsection 2.4 . integral expressions can be found for $(\operatorname{deg}+d)_{Z_{1}}^{-1}$ and $(\operatorname{deg}+d)_{Z_{2}}^{-1}, d \in \mathbb{Z}$. This shows that the result of application of such operators to $I^{+-}\left(N(Z)^{-1}\right)$ and $I^{-+}\left(N(Z)^{-1}\right)$ is analytic on $\Omega$ as well. Finally, the actions $\pi_{l}^{0} \otimes 1$ and $1 \otimes \pi_{r}^{0}$ of $\mathfrak{g l}\left(2, \mathbb{H}_{\mathbb{C}}\right)$ preserve analyticity of functions. 
Each $f \in \mathcal{H} \mathcal{H}^{\omega}$ is harmonic with respect to $Z_{1}$ and $Z_{2}$ :

$$
\square_{Z_{1}} f\left(Z_{1}, Z_{2}\right)=0=\square_{Z_{2}} f\left(Z_{1}, Z_{2}\right) .
$$

We have the following inclusions:

$$
\mathcal{H} \otimes \mathcal{H} \subset \mathcal{H} \mathcal{H}^{\omega} \subset \mathcal{H} \mathcal{H}
$$

Thus, we can think of $\mathcal{H} \mathcal{H}^{\omega}$ as a completion of $\mathcal{H} \otimes \mathcal{H}$.

Definition 76. We call a function $f \in \mathcal{H H}^{\omega}$ extendable if, for each $Z_{0} \in \mathbb{H}_{\mathbb{C}}^{\times}$, there exists an open neighborhood $V \subset \mathbb{H}_{\mathbb{C}}^{\times} \times \mathbb{H}_{\mathbb{C}}^{\times}$of $\left(Z_{0}, Z_{0}\right)$ and two functions $f^{+}$and $f^{-}$analytic on $V$ such that $f=f^{+}$for all points in $\Lambda^{+} \cap V$ and $f=f^{-}$for all points in $\Lambda^{-} \cap V$.

Informally, extendable functions are functions $f$ whose restrictions $\left.f\right|_{\Lambda^{+}}$and $\left.f\right|_{\Lambda^{-}}$extend analytically across the diagonal in $\mathbb{H}_{\mathbb{C}}^{\times} \times \mathbb{H}_{\mathbb{C}}^{\times}$. Clearly, elements of $\mathcal{H} \otimes \mathcal{H}$ are extendable. Also, the following observation is obvious, but will be used in the future.

Lemma 77. The extendable functions in $\mathcal{H H}^{\omega}$ form a subspace that is invariant under the actions $\pi_{l}^{0} \otimes 1,1 \otimes \pi_{r}^{0}$ and $\pi_{l}^{0} \otimes \pi_{r}^{0}$ of $\mathfrak{g l}\left(2, \mathbb{H}_{\mathbb{C}}\right)$.

Remark 78. We expect all functions in $\mathcal{H H}^{\omega}$ to be extendable.

Lemma 79. For each $f \in \mathcal{W}, I(f)$ is extendable and can be written as a finite linear combination of analytic functions on $\Omega$ that are homogeneous in $Z_{1}$ and $Z_{2}$.

Proof. Recall that $\mathscr{W}=\mathscr{K}^{-} \oplus \mathscr{K}^{0} \oplus \mathscr{K}^{+}$. If $f \in I\left(\mathscr{K}^{-} \oplus \mathcal{K}^{+}\right)$, then $I(f) \in \mathcal{H} \otimes \mathcal{H}$ is extendable and can be written as a finite linear combination of homogeneous functions. On the other hand, expression (62) shows that $I\left(N(Z)^{-1}\right.$ ) is extendable and homogeneous in $Z_{1}$ and $Z_{2}$ (of degree -1 in each variable). Since $N(Z)^{-1}$ generates $\mathcal{K}^{0}$, it follows from Lemma 77 that the result is true for all $f \in \mathscr{K}^{0}$.

Let $f$ be an extendable function. Even though it may be singular along the diagonal, we still can construct two analytic functions on $\mathbb{H}_{\mathbb{C}}^{\times}$:

$$
\begin{aligned}
& \left(\operatorname{Diag}^{+} f\right)(Z)=\lim _{\substack{Z_{1}, Z_{2} \rightarrow Z \\
\left(Z_{1}, Z_{2}\right) \in \Lambda^{+}}} f\left(Z_{1}, Z_{2}\right) \quad \text { and } \\
& \left(\operatorname{Diag}^{-} f\right)(Z)=\lim _{\substack{Z_{1}, Z_{2} \rightarrow Z \\
\left(Z_{1}, Z_{2}\right) \in \Lambda^{-}}} f\left(Z_{1}, Z_{2}\right) .
\end{aligned}
$$

In some cases, applying $\mathrm{Diag}^{+}$and $\mathrm{Diag}^{-}$may yield different results, we will see a concrete example in Proposition 87. Note also that functions $\mathrm{Diag}^{+} f$ and $\mathrm{Diag}^{-} f$ need not be elements of $\mathscr{\nVdash}$ because they may not be polynomials on $\mathbb{H}_{\mathbb{C}}^{\times}$. Nevertheless, the operators $\mathrm{Diag}^{+}$and $\mathrm{Diag}^{-}$ intertwine the $\mathfrak{g l}\left(2, \mathbb{H}_{\mathbb{C}}\right)$-actions $\pi_{l}^{0} \otimes \pi_{r}^{0}$ on extendable functions in $\mathcal{H} \mathcal{H}^{\omega}$ and $\rho_{1}$ on $\mathbb{C}$-valued analytic functions on $\mathbb{H}_{\mathbb{C}}^{\times}$.

Lemma 80. If $f\left(Z_{1}, Z_{2}\right) \in \mathcal{H} \otimes \mathcal{H}$, then

$$
\operatorname{Diag}^{+} f=\operatorname{Diag}^{-} f \quad \in \mathscr{K} .
$$

Proof. Note that, when restricted to $\mathcal{H} \otimes \mathcal{H}$, both $\operatorname{Diag}^{+}$and $\operatorname{Diag}^{-}$reduce to the multiplication $\operatorname{map} \mathcal{H} \otimes \mathcal{H} \rightarrow \mathscr{K}$.

Theorem 81. Let $f, g \in \mathscr{K}$, then $I(f) * I(g)$ is extendable and

$$
\operatorname{Diag}^{+}(I(f) * I(g)), \quad \operatorname{Diag}^{-}(I(f) * I(g)) \quad \in \mathscr{W} .
$$


Proof. Recall that $\mathscr{T}=\mathscr{K}^{-} \oplus \mathcal{K}^{0} \oplus \mathcal{K}^{+}$. If $f$ or $g \in \mathscr{K}^{-} \oplus \mathcal{K}^{+}$, then $I(f)$ or $I(g) \in \mathcal{H} \otimes \mathcal{H}$. Then, by Lemma 68, $I(f) * I(g) \in \mathcal{H} \otimes \mathcal{H}$, hence $I(f) * I(g)$ is extendable and (71) is true.

It remains to consider the case $f, g \in \mathscr{K}^{0}$. Since $\mathscr{K}^{0}$ is irreducible and generated by $N(Z)^{-1}$, the result follows from Proposition 71 and Lemmas 69, 77 and 82 .

Lemma 82. For each $d \in \mathbb{Z},(\operatorname{deg}+d)_{Z_{1}}^{-1} I\left(N(Z)^{-1}\right)$ and $(\operatorname{deg}+d)_{Z_{2}}^{-1} I\left(N(Z)^{-1}\right)$ are extendable. Furhermore,

$$
\begin{array}{cc}
\operatorname{Diag}^{+}\left((\operatorname{deg}+d)_{Z_{1}}^{-1} I\left(N(Z)^{-1}\right)\right), & \operatorname{Diag}^{-}\left((\operatorname{deg}+d)_{Z_{1}}^{-1} I\left(N(Z)^{-1}\right)\right), \\
\operatorname{Diag}^{+}\left((\operatorname{deg}+d)_{Z_{2}}^{-1} I\left(N(Z)^{-1}\right)\right), & \operatorname{Diag}^{-}\left((\operatorname{deg}+d)_{Z_{2}}^{-1} I\left(N(Z)^{-1}\right)\right)
\end{array}
$$

are proportional to $N(Z)^{-1}$, and hence elements of $\mathcal{X}$.

Proof. First, we consider the case $d=1$. Recall the dilogarithm function:

$$
\operatorname{Li}_{2}(z)=\sum_{k=1}^{\infty} \frac{z^{k}}{k^{2}},
$$

it extends to an analytic function on $\mathbb{C}$ with a cut along $[1, \infty)$. It also satisfies a well known identity

$$
\mathrm{Li}_{2}(z)+\mathrm{Li}_{2}(1 / z)=-\frac{\pi^{2}}{6}-\frac{1}{2} \log ^{2}(-z)
$$

(see, for example, [Z]). As we saw in the proof of Proposition 71

$$
\begin{aligned}
& I^{+-}\left(N(Z)^{-1}\right)\left(Z_{1}, Z_{2}\right)=\sum_{l, m, n} \frac{N\left(Z_{2}\right)^{-1}}{2 l+1} t_{n \underline{m}}^{l}\left(Z_{1}\right) \cdot t_{m \underline{n}}^{l}\left(Z_{2}^{-1}\right), \\
& I^{-+}\left(N(Z)^{-1}\right)\left(Z_{1}, Z_{2}\right)=\sum_{l, m, n} \frac{N\left(Z_{1}\right)^{-1}}{(2 l+1)^{2}} t_{m \underline{n}}^{l}\left(Z_{1}^{-1}\right) \cdot t_{n \underline{m}}^{l}\left(Z_{2}\right) .
\end{aligned}
$$

We have:

$$
\begin{aligned}
(\operatorname{deg}+1)_{Z_{1}}^{-1} I^{+-}\left(N(Z)^{-1}\right)\left(Z_{1}, Z_{2}\right)=-(\operatorname{deg}+1)_{Z_{2}}^{-1} I^{+-}\left(N(Z)^{-1}\right)\left(Z_{1}, Z_{2}\right) & \\
& =\sum_{l, m, n} \frac{N\left(Z_{2}\right)^{-1}}{(2 l+1)^{2}} t_{n \underline{m}}^{l}\left(Z_{1}\right) \cdot t_{m \underline{n}}^{l}\left(Z_{2}^{-1}\right)
\end{aligned}
$$

Recall that $\lambda_{1}$ and $\lambda_{2}$ are the eigenvalues of $Z_{1} Z_{2}^{-1}$. Then, following calculations (36) from [FL3],

$$
\begin{aligned}
(\operatorname{deg}+1)_{Z_{1}}^{-1} I^{+-}\left(N(Z)^{-1}\right)\left(Z_{1}, Z_{2}\right) & =-(\operatorname{deg}+1)_{Z_{2}}^{-1} I^{+-}\left(N(Z)^{-1}\right)\left(Z_{1}, Z_{2}\right) \\
& =\sum_{l} \frac{N\left(Z_{2}\right)^{-1}}{(2 l+1)^{2}} \frac{\lambda_{1}^{2 l+1}-\lambda_{2}^{2 l+1}}{\lambda_{1}-\lambda_{2}}=\frac{1}{N\left(Z_{2}\right)} \frac{\operatorname{Li}_{2}\left(\lambda_{1}\right)-\operatorname{Li}_{2}\left(\lambda_{2}\right)}{\lambda_{1}-\lambda_{2}} .
\end{aligned}
$$

Similarly,

$$
\begin{aligned}
&(\operatorname{deg}+1)_{Z_{1}}^{-1} I^{-+}\left(N(Z)^{-1}\right)\left(Z_{1}, Z_{2}\right)=-(\operatorname{deg}+1)_{Z_{2}}^{-1} I^{-+}\left(N(Z)^{-1}\right)\left(Z_{1}, Z_{2}\right) \\
&=-\sum_{l, m, n} \frac{N\left(Z_{1}\right)^{-1}}{(2 l+1)^{2}} t_{m}^{l}{ }_{m}\left(Z_{1}^{-1}\right) \cdot t_{n \underline{m}}^{l}\left(Z_{2}\right)=-\sum_{l} \frac{N\left(Z_{1}\right)^{-1}}{(2 l+1)^{2}} \frac{\lambda_{1}^{-(2 l+1)}-\lambda_{2}^{-(2 l+1)}}{\lambda_{1}^{-1}-\lambda_{2}^{-1}} \\
&=-\frac{1}{N\left(Z_{1}\right)} \frac{\operatorname{Li}_{2}\left(\lambda_{1}^{-1}\right)-\operatorname{Li}_{2}\left(\lambda_{2}^{-1}\right)}{\lambda_{1}^{-1}-\lambda_{2}^{-1}}=\frac{1}{N\left(Z_{2}\right)} \frac{\operatorname{Li}_{2}\left(\lambda_{1}^{-1}\right)-\mathrm{Li}_{2}\left(\lambda_{2}^{-1}\right)}{\lambda_{1}-\lambda_{2}}
\end{aligned}
$$


(recall that $\left.N\left(Z_{1}\right)=\lambda_{1} \lambda_{2} \cdot N\left(Z_{2}\right)\right)$. Thus,

$$
\begin{gathered}
(\operatorname{deg}+1)_{Z_{1}}^{-1} I\left(N(Z)^{-1}\right)\left(Z_{1}, Z_{2}\right)=-(\operatorname{deg}+1)_{Z_{2}}^{-1} I\left(N(Z)^{-1}\right)\left(Z_{1}, Z_{2}\right) \\
=-\frac{1}{N\left(Z_{2}\right)} \frac{\operatorname{Li}_{2}\left(\lambda_{1}\right)+\operatorname{Li}_{2}\left(\lambda_{1}^{-1}\right)-\operatorname{Li}_{2}\left(\lambda_{2}\right)-\operatorname{Li}_{2}\left(\lambda_{2}^{-1}\right)}{\lambda_{1}-\lambda_{2}} \\
=\frac{1}{N\left(Z_{2}\right)} \frac{\log ^{2}\left(-\lambda_{1}\right)-\log ^{2}\left(-\lambda_{2}\right)}{2\left(\lambda_{1}-\lambda_{2}\right)} .
\end{gathered}
$$

If $\lambda_{1}=\lambda_{2}=\lambda \neq 1$, we get

$$
\frac{1}{N\left(Z_{2}\right)} \frac{\log (-\lambda)}{\lambda}
$$

This shows that $(\operatorname{deg}+1)_{Z_{1}}^{-1} I\left(N(Z)^{-1}\right)$ and $(\operatorname{deg}+1)_{Z_{2}}^{-1} I\left(N(Z)^{-1}\right)$ are extendable and applying Diag $^{+}$or Diag ${ }^{-}$results in scalar multiples of $N(Z)^{-1}$.

Now, suppose that $d>1$. Consider a power series

$$
p(z)=\sum_{k=1}^{\infty} \frac{z^{k}}{k(k+d-1)} .
$$

Then

$$
\left(z^{d-1} p(z)\right)^{\prime}=\sum_{k=1}^{\infty} \frac{z^{k+d-2}}{k}=-z^{d-2} \log (1-z),
$$

and

$$
\begin{array}{r}
p(z)=-z^{1-d} \int_{0}^{z} t^{d-2} \log (1-t) d t=-\frac{z^{1-d}}{d-1}\left(z^{d-1} \log (1-z)-\int_{0}^{z} \frac{t^{d-1}}{t-1} d t\right) \\
=\frac{1}{d-1}\left(z^{1-d}-1\right) \log (1-z)+\frac{1}{d-1} \sum_{k=1}^{d-1} \frac{z^{k-d+1}}{k} .
\end{array}
$$

Similarly, consider another power series

$$
q(z)=\sum_{k=d}^{\infty} \frac{z^{k}}{k(k+1-d)}
$$

Then

$$
q^{\prime}(z)=\sum_{k=d}^{\infty} \frac{z^{k-1}}{k+1-d}=-z^{d-2} \log (1-z),
$$

and

$$
q(z)=\frac{1}{d-1}\left(1-z^{d-1}\right) \log (1-z)+\frac{1}{d-1} \sum_{k=1}^{d-1} \frac{z^{k}}{k} .
$$

We have:

$$
\begin{aligned}
(\operatorname{deg}+d)_{Z_{1}}^{-1} I^{+-}\left(N(Z)^{-1}\right)\left(Z_{1}, Z_{2}\right) & =\sum_{l, m, n} \frac{N\left(Z_{2}\right)^{-1}}{(2 l+1)(2 l+d)} t_{n \underline{m}}^{l}\left(Z_{1}\right) \cdot t_{m \underline{n}}^{l}\left(Z_{2}^{-1}\right) \\
= & \sum_{l} \frac{N\left(Z_{2}\right)^{-1}}{(2 l+1)(2 l+d)} \frac{\lambda_{1}^{2 l+1}-\lambda_{2}^{2 l+1}}{\lambda_{1}-\lambda_{2}}=\frac{1}{N\left(Z_{2}\right)} \frac{p\left(\lambda_{1}\right)-p\left(\lambda_{2}\right)}{\lambda_{1}-\lambda_{2}} .
\end{aligned}
$$


Similarly,

$$
\begin{aligned}
(\operatorname{deg}+d)_{Z_{1}}^{-1} I^{-+}\left(N(Z)^{-1}\right)\left(Z_{1}, Z_{2}\right) & =-\sum_{l, m, n} \frac{N\left(Z_{1}\right)^{-1}}{(2 l+1)(2 l+2-d)} t_{m \underline{n}}^{l}\left(Z_{1}^{-1}\right) \cdot t_{n \underline{m}}^{l}\left(Z_{2}\right) \\
=-\sum_{l>d / 2-1} \frac{N\left(Z_{1}\right)^{-1}}{(2 l+1)(2 l+2-d)} \frac{\lambda_{1}^{-(2 l+1)}-\lambda_{2}^{-(2 l+1)}}{\lambda_{1}^{-1}-\lambda_{2}^{-1}} & \\
= & -\frac{1}{N\left(Z_{1}\right)} \frac{q\left(\lambda_{1}^{-1}\right)-q\left(\lambda_{2}^{-1}\right)}{\lambda_{1}^{-1}-\lambda_{2}^{-1}}=\frac{1}{N\left(Z_{2}\right)} \frac{q\left(\lambda_{1}^{-1}\right)-q\left(\lambda_{2}^{-1}\right)}{\lambda_{1}-\lambda_{2}}
\end{aligned}
$$

(recall that $\left.N\left(Z_{1}\right)=\lambda_{1} \lambda_{2} \cdot N\left(Z_{2}\right)\right)$. Using

$$
\log (1-\lambda)-\log \left(1-\lambda^{-1}\right)=\log (-\lambda),
$$

we obtain

$$
\begin{gathered}
(\operatorname{deg}+d)_{Z_{1}}^{-1} I\left(N(Z)^{-1}\right)\left(Z_{1}, Z_{2}\right)=-\frac{1}{N\left(Z_{2}\right)} \frac{p\left(\lambda_{1}\right)+q\left(\lambda_{1}^{-1}\right)-p\left(\lambda_{2}\right)-q\left(\lambda_{2}^{-1}\right)}{\lambda_{1}-\lambda_{2}} \\
=\frac{1}{N\left(Z_{2}\right)} \frac{\left(1-\lambda_{1}^{1-d}\right) \log \left(-\lambda_{1}\right)-\left(1-\lambda_{2}^{1-d}\right) \log \left(-\lambda_{2}\right)}{(d-1)\left(\lambda_{1}-\lambda_{2}\right)} \\
-\frac{N\left(Z_{2}\right)^{-1}}{d-1} \sum_{k=1}^{d-1} \frac{\lambda_{1}^{k-d+1}+\lambda_{1}^{-k}-\lambda_{2}^{k-d+1}-\lambda_{2}^{-k}}{k\left(\lambda_{1}-\lambda_{2}\right)} .
\end{gathered}
$$

In the same fashion we also obtain:

$$
\begin{gathered}
(\operatorname{deg}+d)_{Z_{2}}^{-1} I\left(N(Z)^{-1}\right)\left(Z_{1}, Z_{2}\right)=-\frac{1}{N\left(Z_{2}\right)} \frac{q\left(\lambda_{1}\right)+p\left(\lambda_{1}^{-1}\right)-q\left(\lambda_{2}\right)-p\left(\lambda_{2}^{-1}\right)}{\lambda_{1}-\lambda_{2}} \\
=\frac{1}{N\left(Z_{2}\right)} \frac{\left(\lambda_{1}^{d-1}-1\right) \log \left(-\lambda_{1}\right)-\left(\lambda_{2}^{d-1}-1\right) \log \left(-\lambda_{2}\right)}{(d-1)\left(\lambda_{1}-\lambda_{2}\right)} \\
-\frac{N\left(Z_{2}\right)^{-1}}{d-1} \sum_{k=1}^{d-1} \frac{\lambda_{1}^{k}+\lambda_{1}^{d-k-1}-\lambda_{2}^{k}-\lambda_{2}^{d-k-1}}{k\left(\lambda_{1}-\lambda_{2}\right)} .
\end{gathered}
$$

Then equations (73) and (174) show that $(\operatorname{deg}+d)_{Z_{1}}^{-1} I\left(N(Z)^{-1}\right)$ and $(\operatorname{deg}+d)_{Z_{2}}^{-1} I\left(N(Z)^{-1}\right)\left(Z_{1}, Z_{2}\right)$ are extendable and applying $\operatorname{Diag}^{+}$or Diag $^{-}$results in scalar multiples of $N(Z)^{-1}$. The case $d<1$ is similar. follows.

Theorem 81 allows us to define two $\mathfrak{g l}\left(2, \mathbb{H}_{\mathbb{C}}\right)$-invariant multiplication operations on $\nVdash$ as

Definition 83. Let $f, g \in \mathscr{T}$, define

$$
\begin{aligned}
& f *^{+} g=\operatorname{Diag}^{+}(I(f) * I(g)), \\
& f *^{-} g=\operatorname{Diag}^{-}(I(f) * I(g)) .
\end{aligned}
$$

Lemma 84. The two multiplication operations are related to each other as follows:

$$
\begin{aligned}
& f *^{+} g=f *^{-} g \quad \text { if } f \text { or } g \text { is in } \mathscr{K}^{-} \oplus \mathcal{K}^{+}, \\
& N(Z)^{-1} *^{+} N(Z)^{-1}=-N(Z)^{-1} *^{-} N(Z)^{-1}=-\pi i \cdot N(Z)^{-1} .
\end{aligned}
$$


Proof. If $f$ or $g \in \mathscr{K}^{-} \oplus \mathscr{K}^{+}$, then $I(f)$ or $I(g) \in \mathcal{H} \otimes \mathcal{H}$. Then, by Lemma 68, $I(f) * I(g) \in \mathcal{H} \otimes \mathcal{H}$ and, by Lemma 80 .

$$
\operatorname{Diag}^{+}(I(f) * I(g))=\operatorname{Diag}^{-}(I(f) * I(g)) .
$$

On the other hand, Proposition 71 and our previous calculations (172) show that

$$
\begin{aligned}
& \operatorname{Diag}^{+}\left(I N(Z)^{-1} * I N(Z)^{-1}\right)=\lim _{\substack{Z_{1}, Z_{2} \rightarrow Z \\
\left(Z_{1}, Z_{2}\right) \in \Lambda^{+}}}(\operatorname{deg}+1)_{Z_{1}}^{-1} I\left(N(Z)^{-1}\right)\left(Z_{1}, Z_{2}\right)=-\frac{\pi i}{N(Z)}, \\
& \operatorname{Diag}^{-}\left(I N(Z)^{-1} * I N(Z)^{-1}\right)=\lim _{\substack{Z_{1}, Z_{2} \rightarrow Z \\
\left(Z_{1}, Z_{2}\right) \in \Lambda^{-}}}(\operatorname{deg}+1)_{Z_{1}}^{-1} I\left(N(Z)^{-1}\right)\left(Z_{1}, Z_{2}\right)=\frac{\pi i}{N(Z)} .
\end{aligned}
$$

Proposition 85. One can also consider multiplications obtained by taking linear combinations of $f *^{+} g$ and $f *^{-} g$. Thus we obtain a one-parameter family of $\mathfrak{g l}\left(2, \mathbb{H}_{\mathbb{C}}\right)$-invariant multiplications on $\nVdash$.

Example 86. In this example we show that the multiplication operations $*^{+}$and $*^{-}$on $\mathscr{\nVdash}$ are not associative.

Proof. Consider elements $f_{1}(Z)=N(Z)^{-1}, f_{2}(Z)=1$ and $f_{3}(Z)=N(Z)^{-2}$ of $\mathscr{K}$. Then

$$
I\left(f_{2}\right)=1, \quad I\left(f_{3}\right)=N\left(Z_{1}\right)^{-1} \cdot N\left(Z_{2}\right)^{-1} .
$$

By Proposition 71 ,

$$
I\left(f_{1}\right) * I\left(f_{2}\right)=1, \quad I\left(f_{2}\right) * I\left(f_{3}\right)=N\left(Z_{2}\right)^{-1}, \quad I\left(f_{1}\right) * I\left(f_{2}\right) * I\left(f_{3}\right)=N\left(Z_{2}\right)^{-1} .
$$

Hence

$$
f_{1} *^{ \pm} f_{2}=1, \quad f_{2} *^{ \pm} f_{3}=N(Z)^{-1}, \quad\left(f_{1} *^{ \pm} f_{2}\right) *^{ \pm} f_{3}=N(Z)^{-1} .
$$

On the other hand, by Lemma 84 ,

$$
f_{1} *^{+}\left(f_{2} *^{ \pm} f_{3}\right)=-\pi i \cdot N(Z)^{-1}, \quad f_{1} *^{-}\left(f_{2} *^{ \pm} f_{3}\right)=\pi i \cdot N(Z)^{-1} .
$$

We also have

$$
\operatorname{Diag}^{+}\left(I\left(f_{1}\right) * I\left(f_{2}\right) * I\left(f_{3}\right)\right)=\operatorname{Diag}^{-}\left(I\left(f_{1}\right) * I\left(f_{2}\right) * I\left(f_{3}\right)\right)=N(Z)^{-1} .
$$

Proposition 87. For each positive integer n,

$$
\underbrace{I\left(N(Z)^{-1}\right) * \cdots * I\left(N(Z)^{-1}\right)}_{n \text { times }} \in \mathcal{H H}^{\omega}
$$

is extendable and

$$
\begin{aligned}
& \operatorname{Diag}^{+}(\underbrace{I\left(N(Z)^{-1}\right) * \cdots * I\left(N(Z)^{-1}\right)}_{n \text { times }})=\kappa_{n}^{+} \cdot N(Z)^{-1}, \\
& \operatorname{Diag}^{-}(\underbrace{I\left(N(Z)^{-1}\right) * \cdots * I\left(N(Z)^{-1}\right)}_{n \text { times }})=\kappa_{n}^{-} \cdot N(Z)^{-1},
\end{aligned}
$$

where

$$
\kappa_{n}^{+}=-(2 \pi i)^{n-1} \frac{B_{n-1}(1)}{(n-1) !}, \quad \kappa_{n}^{-}=-(2 \pi i)^{n-1} \frac{B_{n-1}(0)}{(n-1) !} .
$$


Proof. Recall the polylogarithm function:

$$
\operatorname{Li}_{n}(z)=\sum_{k=1}^{\infty} \frac{z^{k}}{k^{n}}
$$

it extends to an analytic function on $\mathbb{C}$ with a cut along $[1, \infty)$. It also satisfies an identity

$$
\operatorname{Li}_{n}(z)+(-1)^{n} \operatorname{Li}_{n}(1 / z)=-\frac{(2 \pi i)^{n}}{n !} B_{n}\left(\frac{1}{2}-\frac{\log (-z)}{2 \pi i}\right), \quad z \notin(0, \infty),
$$

where $B_{n}$ are the Bernoulli polynomials (equation 1.11(18) in Er as well as its correction on Wikipedia's Polylogarithm page).

By Proposition 71, we have:

$$
\begin{aligned}
& \underbrace{I\left(N(Z)^{-1}\right) * \cdots * I\left(N(Z)^{-1}\right)}_{n-1 \text { times }} * I^{+-}\left(N(Z)^{-1}\right) \\
& =\underbrace{(\operatorname{deg}+1)_{Z_{1}}^{-1} \cdots(\operatorname{deg}+1)_{Z_{1}}^{-1}}_{n-1 \text { times }} \sum_{l, m, n} \frac{N\left(Z_{2}\right)^{-1}}{2 l+1} t_{n \underline{m}}^{l}\left(Z_{1}\right) \cdot t_{m \underline{n}}^{l}\left(Z_{2}^{-1}\right) \\
& =\sum_{l} \frac{N\left(Z_{2}\right)^{-1}}{(2 l+1)^{n}} \frac{\lambda_{1}^{2 l+1}-\lambda_{2}^{2 l+1}}{\lambda_{1}-\lambda_{2}}=\frac{1}{N\left(Z_{2}\right)} \frac{\operatorname{Li}_{n}\left(\lambda_{1}\right)-\operatorname{Li}_{n}\left(\lambda_{2}\right)}{\lambda_{1}-\lambda_{2}}
\end{aligned}
$$

Similarly,

$$
\begin{aligned}
& \underbrace{I\left(N(Z)^{-1}\right) * \cdots * I\left(N(Z)^{-1}\right)}_{n-1 \text { times }} * I^{+-}\left(N(Z)^{-1}\right) \\
& =\underbrace{(\operatorname{deg}+1)_{Z_{1}}^{-1} \cdots(\operatorname{deg}+1)_{Z_{1}}^{-1}}_{n-1 \text { times }} \sum_{l, m, n} \frac{N\left(Z_{1}\right)^{-1}}{2 l+1} t_{m \underline{n}}^{l}\left(Z_{1}^{-1}\right) \cdot t_{n \underline{m}}^{l}\left(Z_{2}\right) \\
& =(-1)^{n-1} \sum_{l} \frac{N\left(Z_{1}\right)^{-1}}{(2 l+1)^{n}} \frac{\lambda_{1}^{-(2 l+1)}-\lambda_{2}^{-(2 l+1)}}{\lambda_{1}^{-1}-\lambda_{2}^{-1}}=\frac{(-1)^{n-1}}{N\left(Z_{1}\right)} \frac{\operatorname{Li}_{n}\left(\lambda_{1}^{-1}\right)-\operatorname{Li}_{n}\left(\lambda_{2}^{-1}\right)}{\lambda_{1}^{-1}-\lambda_{2}^{-1}} \\
& =\frac{(-1)^{n}}{N\left(Z_{2}\right)} \frac{\operatorname{Li}_{n}\left(\lambda_{1}^{-1}\right)-\operatorname{Li}_{n}\left(\lambda_{2}^{-1}\right)}{\lambda_{1}-\lambda_{2}}
\end{aligned}
$$

(recall that $\left.N\left(Z_{1}\right)=\lambda_{1} \lambda_{2} \cdot N\left(Z_{2}\right)\right)$. Thus,

$$
\begin{aligned}
& \underbrace{I\left(N(Z)^{-1}\right) * \cdots * I\left(N(Z)^{-1}\right)}_{n \text { times }} \\
& =-\frac{1}{N\left(Z_{2}\right)} \frac{\operatorname{Li}_{n}\left(\lambda_{1}\right)+(-1)^{n} \operatorname{Li}_{n}\left(\lambda_{1}^{-1}\right)-\operatorname{Li}_{n}\left(\lambda_{2}\right)-(-1)^{n} \operatorname{Li}_{n}\left(\lambda_{2}^{-1}\right)}{\lambda_{1}-\lambda_{2}} \\
& =\frac{(2 \pi i)^{n}}{n ! N\left(Z_{2}\right)} \frac{B_{n}\left(\frac{1}{2}-\frac{\log \left(-\lambda_{1}\right)}{2 \pi i}\right)-B_{n}\left(\frac{1}{2}-\frac{\log \left(-\lambda_{2}\right)}{2 \pi i}\right)}{\lambda_{1}-\lambda_{2}}
\end{aligned}
$$

If $\lambda_{1}, \lambda_{2} \rightarrow 1$ with $\operatorname{sign}\left(\operatorname{Im} \lambda_{1}\right), \operatorname{sign}\left(\operatorname{Im} \lambda_{2}\right)>0$,

$$
\frac{(2 \pi i)^{n}}{n ! N\left(Z_{2}\right)} \frac{B_{n}\left(\frac{1}{2}-\frac{\log \left(-\lambda_{1}\right)}{2 \pi i}\right)-B_{n}\left(\frac{1}{2}-\frac{\log \left(-\lambda_{2}\right)}{2 \pi i}\right)}{\lambda_{1}-\lambda_{2}} \rightarrow-(2 \pi i)^{n-1} \frac{B_{n}^{\prime}(1)}{n !} \frac{1}{N\left(Z_{2}\right)} ;
$$


and if $\lambda_{1}, \lambda_{2} \rightarrow 1$ with $\operatorname{sign}\left(\operatorname{Im} \lambda_{1}\right), \operatorname{sign}\left(\operatorname{Im} \lambda_{2}\right)<0$,

$$
\frac{(2 \pi i)^{n}}{n ! N\left(Z_{2}\right)} \frac{B_{n}\left(\frac{1}{2}-\frac{\log \left(-\lambda_{1}\right)}{2 \pi i}\right)-B_{n}\left(\frac{1}{2}-\frac{\log \left(-\lambda_{2}\right)}{2 \pi i}\right)}{\lambda_{1}-\lambda_{2}} \rightarrow-(2 \pi i)^{n-1} \frac{B_{n}^{\prime}(0)}{n !} \frac{1}{N\left(Z_{2}\right)} .
$$

Since the Bernoulli polynomials satisfy

$$
B_{n}^{\prime}(z)=n B_{n-1}(z)
$$

(see, for example, [Er]), the result follows.

Remark 88. It is well known (see, for example, [Er]) that $B_{n}(0)$ is the $n$-th Bernoulli number $B_{n}$ and that

$$
\begin{gathered}
B_{n}(1)=B_{n}(0), \quad n \geq 2, \\
B_{0}(0)=B_{0}(1)=1, \quad B_{1}(0)=-1 / 2, \quad B_{1}(1)=1 / 2, \\
B_{2 n+1}=0, \quad n=1,2,3, \ldots
\end{gathered}
$$

\subsection{Convolution Algebra}

Recall the bases of left and right regular polynomial functions on $\mathbb{H}_{\mathbb{C}}^{\times}$with values in $\mathbb{S}$ and $\mathbb{S}^{\prime}$ respectively that were introduced in Proposition 24 of [FL1]:

$$
\begin{aligned}
& v_{l, m, n}^{+}(Z)=\left(\begin{array}{c}
\left(l-m+\frac{1}{2}\right) t_{n}^{l} \frac{m+\frac{1}{2}}{2}(Z) \\
\left(l+m+\frac{1}{2}\right) t_{n \frac{m-\frac{1}{2}}{l}}(Z)
\end{array}\right), \\
& v_{l, m, n}^{-}(Z)=\left(\begin{array}{c}
\left(l-m+\frac{1}{2}\right) N \overline{(Z)^{-1}} \cdot t_{m-\frac{1}{2} \underline{n}}^{l}\left(Z^{-1}\right) \\
\left(l+m+\frac{1}{2}\right) N(Z)^{-1} \cdot t_{m+\frac{1}{2} \underline{n}}^{l}\left(Z^{-1}\right)
\end{array}\right), \\
& v_{l, m, n}^{\prime+}(Z)=\left(t_{n+\frac{1}{2} \underline{m}}^{l-\frac{1}{2}}(Z), t_{n-\frac{1}{2} \underline{m}}^{l-\frac{1}{2}}(Z)\right), \\
& v_{l, m, n}^{\prime-}(Z)=\left(N(Z)^{-1} \cdot t_{m \underline{n-\frac{1}{2}}}^{l+\frac{1}{2}}\left(Z^{-1}\right), N(Z)^{-1} \cdot t_{m \underline{m+\frac{1}{2}}}^{l+\frac{1}{2}}\left(Z^{-1}\right)\right) \\
& l=0, \frac{1}{2}, 1, \frac{3}{2}, \ldots, \\
& \begin{array}{c}
m=-l-\frac{1}{2},-l+\frac{3}{2}, \ldots, l+\frac{1}{2}, \\
\quad n=-l,-l+1, \ldots, l ;
\end{array} \\
& l=\frac{1}{2}, 1, \frac{3}{2}, 2, \ldots, \\
& m=-l+\frac{1}{2},-l+\frac{3}{2}, \ldots, l-\frac{1}{2}, \\
& n=-l,-l+1, \ldots, l \text {; } \\
& l=\frac{1}{2}, 1, \frac{3}{2}, 2, \ldots, \\
& \begin{array}{c}
m=-l+\frac{1}{2},-l+\frac{3}{2}, \ldots, l-\frac{1}{2}, \\
n=-l,-l+1, \ldots, l ;
\end{array} \\
& l=0, \frac{1}{2}, 1, \frac{3}{2}, \ldots, \\
& \begin{array}{c}
m=-l-\frac{1}{2},-l+\frac{3}{2}, \ldots, l+\frac{1}{2}, \\
n=-l,-l+1, \ldots, l .
\end{array}
\end{aligned}
$$

Then

$$
\begin{aligned}
& \mathcal{V}^{+}=\left\{f \in \mathbb{S} \otimes \mathbb{C}\left[z_{11}, z_{12}, z_{21}, z_{22}\right] ; \nabla^{+} f=0\right\} \\
& =\mathbb{C} \text {-span of }\left\{v_{l, m, n}^{+}(Z) ; \begin{array}{c}
l=0, \frac{1}{2}, 1, \frac{3}{2}, \ldots, \\
m=-l-\frac{1}{2},-l+\frac{3}{2}, \ldots, l+\frac{1}{2}, \\
n=-l,-l+1, \ldots, l
\end{array}\right\} \text {, } \\
& \mathcal{V}^{-}=\left\{f \in \mathbb{S} \otimes \mathbb{C}\left[z_{11}, z_{12}, z_{21}, z_{22}, N(Z)^{-1}\right] ; N(Z)^{-1} \cdot Z^{-1} \cdot f\left(Z^{-1}\right) \in \mathcal{V}^{+}\right\} \\
& =\mathbb{C} \text {-span of }\left\{v_{l, m, n}^{-}(Z) ; \begin{array}{c}
l=\frac{1}{2}, 1, \frac{3}{2}, 2, \ldots, \\
m=-l+\frac{1}{2},-l+\frac{3}{2}, \ldots, l-\frac{1}{2}, \\
n=-l,-l+1, \ldots, l
\end{array}\right\}, \\
& \mathcal{V}=\left\{f \in \mathbb{S} \otimes \mathbb{C}\left[z_{11}, z_{12}, z_{21}, z_{22}, N(Z)^{-1}\right] ; \nabla^{+} f=0\right\} \\
& =\mathcal{V}^{+} \oplus \mathcal{V}^{-}
\end{aligned}
$$




$$
\begin{aligned}
\mathcal{V}^{\prime+} & =\left\{g \in \mathbb{S}^{\prime} \otimes \mathbb{C}\left[z_{11}, z_{12}, z_{21}, z_{22}\right] ; g \nabla^{+}=0\right\} \\
& =\mathbb{C} \text {-span of }\left\{v_{l, m, n}^{\prime+}(Z) ; \begin{array}{c}
l=\frac{1}{2}, 1, \frac{3}{2}, 2, \ldots, \\
m=-l+\frac{1}{2},-l+\frac{3}{2}, \ldots, l-\frac{1}{2}, \\
n=-l,-l+1, \ldots, l
\end{array}\right\}, \\
\mathcal{V}^{\prime-} & =\left\{g \in \mathbb{S}^{\prime} \otimes \mathbb{C}\left[z_{11}, z_{12}, z_{21}, z_{22}, N(Z)^{-1}\right] ; N(Z)^{-1} \cdot g\left(Z^{-1}\right) \cdot Z^{-1} \in \mathcal{V}^{\prime+}\right\} \\
& =\mathbb{C} \text {-span of }\left\{v_{l, m, n}^{\prime-}(Z) ; \begin{array}{c}
l=0, \frac{1}{2}, 1, \frac{3}{2}, \ldots, \\
m=-l-\frac{1}{2},-l+\frac{3}{2}, \ldots, l+\frac{1}{2}, \\
n=-l,-l+1, \ldots, l
\end{array}\right\}, \\
\mathcal{V}^{\prime} & =\left\{g \in \mathbb{S}^{\prime} \otimes \mathbb{C}\left[z_{11}, z_{12}, z_{21}, z_{22}, N(Z)^{-1}\right] ; g \nabla^{+}=0\right\} \\
& =\mathcal{V}^{\prime+} \oplus \mathcal{V}^{\prime-} .
\end{aligned}
$$

The Lie algebra $\mathfrak{g l}\left(2, \mathbb{H}_{\mathbb{C}}\right)$ acts on $\mathcal{V}$ and $\mathcal{V}^{\prime}$ via $\pi_{l}$ and $\pi_{r}$ respectively. There is a non-degenerate $\mathfrak{g l}\left(2, \mathbb{H}_{\mathbb{C}}\right)$-invariant bilinear pairing between $\left(\pi_{r}, \mathcal{V}^{\prime}\right)$ and $\left(\pi_{l}, \mathcal{V}\right)$ given by the integral formula

$$
\langle g, f\rangle_{\mathcal{V}}=\frac{1}{2 \pi^{2}} \int_{Z \in S U(2)} g(Z) \cdot D z \cdot f(Z), \quad g \in \mathcal{V}^{\prime}, f \in \mathcal{V},
$$

(equation (29) in [FL1]). The above basis functions satisfy the following orthogonality relations:

$$
\begin{aligned}
& \left\langle v_{l, m, n}^{--}, v_{l^{\prime}, m^{\prime}, n^{\prime}}^{+}\right\rangle_{\mathcal{V}}=\delta_{l l^{\prime}} \delta_{m m^{\prime}} \delta_{n n^{\prime}} \\
& \left\langle v_{l, m, n}^{+}, v_{l^{\prime}, m^{\prime}, n^{\prime}}^{-}\right\rangle_{\mathcal{V}}=\delta_{l l^{\prime}} \delta_{m m^{\prime}} \delta_{n n^{\prime}} \\
& \left\langle v_{l, m, n}^{\prime+}, v_{l^{\prime}, m^{\prime}, n^{\prime}}^{+}\right\rangle_{\mathcal{V}}=\left\langle v_{l, m, n}^{\prime-}, v_{l^{\prime}, m^{\prime}, n^{\prime}}^{-}\right\rangle_{\mathcal{V}}=0
\end{aligned}
$$

(Proposition 24 in [FL1]).

We consider $\mathcal{V} \otimes \mathcal{V}^{\prime}$. This space consists of polynomial $\mathbb{H}_{\mathbb{C}^{-v} \text {-valued functions in two variables }}$ $Z_{1}, Z_{2} \in \mathbb{H}_{\mathbb{C}}^{\times}$that are left regular with respect to $Z_{1}$ and right regular with respect to $Z_{2}$. We have a multiplication (or restriction to the diagonal) operation

$$
\text { Mult }: \mathcal{V} \otimes \mathcal{V}^{\prime} \rightarrow \mathcal{W}, \quad v\left(Z_{1}\right) \otimes v^{\prime}\left(Z_{2}\right) \mapsto v(Z) \cdot v^{\prime}(Z)
$$

It is clearly $\mathfrak{g l}\left(2, \mathbb{H}_{\mathbb{C}}\right)$-equivariant with respect to the $\pi_{l} \otimes \pi_{r}$ action on $\mathcal{V} \otimes \mathcal{V}^{\prime}$ and $\rho_{2}$ action on $\mathcal{W}$.

Proposition 89. The image under the multiplication map Mult $: \mathcal{V} \otimes \mathcal{V}^{\prime} \rightarrow \mathcal{W}$ is precisely $\mathcal{Q}^{+} \oplus \mathcal{Q}^{0} \oplus \mathcal{Q}^{-}$.

Proof. Multiplying generators of $\mathcal{V}$ and $\mathcal{V}^{\prime}$, one checks that the image of $\mathcal{V} \otimes \mathcal{V}^{\prime}$ contains $\mathcal{Q}^{+}, \mathcal{Q}^{0}$ and $\mathcal{Q}^{-}$. Thus it remains to show the other inclusion. It is easy to see that $\operatorname{Tr} \circ \partial^{+}$annihilates the image, hence $\operatorname{Mult}\left(\mathcal{V} \otimes \mathcal{V}^{\prime}\right) \subset \operatorname{ker}\left(\operatorname{Tr} \circ \partial^{+}\right)$. Recall that the decomposition of $\operatorname{ker}\left(\operatorname{Tr} \circ \partial^{+}\right)$ into irreducible components was obtained in Subsection [5.1, By Theorem 28 from [FL1], $\mathcal{V}, \mathcal{V}^{\prime}$ and hence $\mathcal{V} \otimes \mathcal{V}^{\prime}$ have inner products such that the real form $\mathfrak{u}(2,2)$ of $\mathfrak{g l}\left(2, \mathbb{H}_{\mathbb{C}}\right)$ acts unitarily. In particular, $\left(\pi_{l} \otimes \pi_{r}, \mathcal{V} \otimes \mathcal{V}^{\prime}\right)$ is semisimple. It follows that the other irreducible components of $\operatorname{ker}\left(\operatorname{Tr} \circ \partial^{+}\right)$cannot appear in the image of the multiplication map because they are not semisimple.

We define convolution operation on $\mathcal{V} \otimes \mathcal{V}^{\prime}$ as

$$
(F * G)\left(Z_{1}, Z_{2}\right)=\frac{1}{2 \pi^{2}} \int_{W \in S U(2)} F\left(Z_{1}, W\right) \cdot D w \cdot G\left(W, Z_{2}\right), \quad F, G \in \mathcal{V} \otimes \mathcal{V}^{\prime}
$$

Alternatively, this operation can be defined on pure tensors as follows. If $F\left(Z_{1}, Z_{2}\right)=v\left(Z_{1}\right) \otimes$ $v^{\prime}\left(Z_{2}\right), G\left(Z_{1}, Z_{2}\right)=w\left(Z_{1}\right) \otimes w^{\prime}\left(Z_{2}\right) \in \mathcal{V} \otimes \mathcal{V}^{\prime}$, then

$$
(F * G)\left(Z_{1}, Z_{2}\right)=\left\langle v^{\prime}, w\right\rangle_{\mathcal{V}} \cdot v\left(Z_{1}\right) \otimes w^{\prime}\left(Z_{2}\right) .
$$


This operation gives $\mathcal{V} \otimes \mathcal{V}^{\prime}$ the structure of an associative algebra. Since the bilinear pairing is $\mathfrak{g l}\left(2, \mathbb{H}_{\mathbb{C}}\right)$-invariant, the above convolution product is $\mathfrak{g l}\left(2, \mathbb{H}_{\mathbb{C}}\right)$-equivariant with respect to the $\pi_{l} \otimes \pi_{r}$ action on $\mathcal{V} \otimes \mathcal{V}^{\prime}$.

Next, we consider a space $\mathcal{A}$ consisting of infinite sums $\sum_{i} v_{i}\left(Z_{1}\right) \otimes v_{i}^{\prime}\left(Z_{2}\right)$, where $v_{i} \in \mathcal{V}$, $v_{i}^{\prime} \in \mathcal{V}^{\prime}$, such that

$$
\text { | degree of } v_{i}+\text { degree of } v_{i}^{\prime} \mid
$$

is bounded. More precisely, $\mathcal{A}$ consists of formal series of the form

$$
\begin{aligned}
& \sum_{\substack{l, m, n \\
l^{\prime}, m^{\prime}, n^{\prime}}} a_{\substack{l, m, n \\
l^{\prime}, m^{\prime}, n^{\prime}}} v_{l, m, n}^{+}\left(Z_{1}\right) \cdot v_{l^{\prime}, m^{\prime}, n^{\prime}}^{\prime+}\left(Z_{2}\right)+\sum_{\substack{l, m, n \\
l^{\prime}, m^{\prime}, n^{\prime}}} b_{\substack{l, m, n \\
l^{\prime}, m^{\prime}, n^{\prime}}} v_{l, m, n}^{+}\left(Z_{1}\right) \cdot v_{l^{\prime}, m^{\prime}, n^{\prime}}^{\prime-}\left(Z_{2}\right) \\
& +\sum_{\substack{l, m, n \\
l^{\prime}, m^{\prime}, n^{\prime}}} c_{\substack{l, m, n \\
l^{\prime}, m^{\prime}, n^{\prime}}}^{l, m, n}\left(Z_{1}\right) \cdot v_{l^{\prime}, m^{\prime}, n^{\prime}}^{+}\left(Z_{2}\right)+\sum_{\substack{l, m, n \\
l^{\prime}, m^{\prime}, n^{\prime}}} d_{\substack{l, m, n \\
l^{\prime}, m^{\prime}, n^{\prime}}}^{-} v_{l, m, n}^{-}\left(Z_{1}\right) \cdot v_{l^{\prime}, m^{\prime}, n^{\prime}}^{\prime-}\left(Z_{2}\right)
\end{aligned}
$$

such that only finitely many coefficients $a_{\substack{l, m, n \\ l^{\prime}, m^{\prime}, n^{\prime}}}$ 's, $d_{\substack{l, m, n \\ l^{\prime}, m^{\prime}, n^{\prime}}}$ 's are non-zero and non-zero coefficients $b_{\substack{l, m, n \\ l^{\prime}, m^{\prime}, n^{\prime}}}$ 's, $c_{\substack{l, m, n \\ l^{\prime}, m^{\prime}, n^{\prime}}}$ 's have bounded difference of indices $\left|l-l^{\prime}\right|$. We have an analogue of Lemma 68, its proof is exactly the same.

Lemma 90. The convolution operation (78) extends to $\mathcal{A}$ and gives it the structure of a $\mathfrak{g l}\left(2, \mathbb{H}_{\mathbb{C}}\right)$-invariant associative algebra. Moreover, if $F \in \mathcal{V} \otimes \mathcal{V}^{\prime}$ and $G \in \mathcal{A}$, then both $F * G$ and $G * F$ lie in $\mathcal{V} \otimes \mathcal{V}^{\prime}$

Unlike $\mathcal{V} \otimes \mathcal{V}^{\prime}, \mathcal{A}$ has a unit. The expression for the unit is obtained by formally combining the two matrix coefficient expansions of $\frac{\left(Z_{1}-Z_{2}\right)^{-1}}{N\left(Z_{1}-Z_{2}\right)}$ given in Proposition 26 from [FL1] (see also Proposition 113):

$$
1_{\mathcal{A}}=\sum_{l, m, n} v_{l, m, n}^{+}\left(Z_{1}\right) \cdot v_{l, m, n}^{\prime-}\left(Z_{2}\right)+\sum_{l, m, n} v_{l, m, n}^{-}\left(Z_{1}\right) \cdot v_{l, m, n}^{++}\left(Z_{2}\right) \quad \in \mathcal{A} .
$$

The fact that $1_{\mathcal{A}}$ is indeed a unit follows from the definition of the convolution operation and orthogonality relations (75)-(177).

The Lie algebra $\mathfrak{g l}\left(2, \mathbb{H}_{\mathbb{C}}\right)$ can act on $\mathcal{V} \otimes \mathcal{V}^{\prime}$ by at least three different actions: $\pi_{l} \otimes 1,1 \otimes \pi_{r}$ and $\pi_{l} \otimes \pi_{r}$. Clearly, all three actions extend to $\mathcal{A}$. Then the convolution operation on $\mathcal{A}$ is $\left(\pi_{l} \otimes \pi_{r}\right)$-equivariant.

Similarly to how we did in the case of $\mathcal{H} \mathcal{H}$, we define operators $(\operatorname{deg}+d)_{Z_{1}}^{-1}$ and $(\operatorname{deg}+d)_{Z_{2}}^{-1}$ on $\mathcal{A}$, where $d \in \mathbb{Z}$. If $F \in \mathcal{A}$ is expressed as a series (79), then

$$
\begin{aligned}
& (\operatorname{deg}+d)_{Z_{1}}^{-1} F\left(Z_{1}, Z_{2}\right)=\sum_{\substack{l>-d / 2 \\
m, n, l^{\prime}, m^{\prime}, n^{\prime}}} \frac{a_{l^{\prime}, m, n}^{l, m}, n^{\prime}}{2 l+d} v_{l, m, n}^{+}\left(Z_{1}\right) \cdot v_{l^{\prime}, m^{\prime}, n^{\prime}}^{+}\left(Z_{2}\right) \\
& +\sum_{\substack{l>-d / 2 \\
m, n, l^{\prime}, m^{\prime}, n^{\prime}}} \frac{b_{\substack{l, m, n \\
l^{\prime}, m^{\prime}, n^{\prime}}}}{2 l+d} v_{l, m, n}^{+}\left(Z_{1}\right) \cdot v_{l^{\prime}, m^{\prime}, n^{\prime}}^{\prime-}\left(Z_{2}\right) \\
& -\sum_{\substack{l>d / 2-1 \\
m, n, l^{\prime}, m^{\prime}, n^{\prime}}} \frac{c_{l^{\prime}, m^{\prime}, n^{\prime}}^{l, m, n}}{2 l+2-d} v_{l, m, n}^{-}\left(Z_{1}\right) \cdot v_{l^{\prime}, m^{\prime}, n^{\prime}}^{+}\left(Z_{2}\right) \\
& -\sum_{\substack{l>d / 2-1 \\
m, n, l^{\prime}, m^{\prime}, n^{\prime}}} \frac{d_{l, m, n}^{l, m, n}}{2 l+2-d} v_{l, m, n}^{-}\left(Z_{1}\right) \cdot v_{l^{\prime}, m^{\prime}, n^{\prime}}^{\prime-}\left(Z_{2}\right)
\end{aligned}
$$


Note that if $d \neq 1$ or 2 , certain terms get discarded. Then $(\operatorname{deg}+d)_{Z_{2}}^{-1} F\left(Z_{1}, Z_{2}\right)$ is defined similarly. We have analogues of Lemmas 69 and 70.

Lemma 91. Let $F\left(Z_{1}, Z_{2}\right) \in \mathcal{A}, d \in \mathbb{Z}$ and $A, B, C, D \in \mathbb{H}_{\mathbb{C}}$. We have the following commutation relations:

$$
\begin{aligned}
& (\operatorname{deg}+d)_{Z_{1}}^{-1}\left(\pi_{l} \otimes 1\right)\left(\begin{array}{cc}
A & 0 \\
0 & D
\end{array}\right) F\left(Z_{1}, Z_{2}\right)=\left(\pi_{l} \otimes 1\right)\left(\begin{array}{cc}
A & 0 \\
0 & D
\end{array}\right)(\operatorname{deg}+d)_{Z_{1}}^{-1} F\left(Z_{1}, Z_{2}\right), \\
& (\operatorname{deg}+d)_{Z_{1}}^{-1}\left(\pi_{l} \otimes 1\right)\left(\begin{array}{cc}
0 & B \\
0 & 0
\end{array}\right) F\left(Z_{1}, Z_{2}\right)=\left(\pi_{l} \otimes 1\right)\left(\begin{array}{cc}
0 & B \\
0 & 0
\end{array}\right)(\operatorname{deg}+d-1)_{Z_{1}}^{-1} F\left(Z_{1}, Z_{2}\right), \\
& (\operatorname{deg}+d)_{Z_{1}}^{-1}\left(\pi_{l} \otimes 1\right)\left(\begin{array}{cc}
0 & 0 \\
C & 0
\end{array}\right) F\left(Z_{1}, Z_{2}\right)=\left(\pi_{l} \otimes 1\right)\left(\begin{array}{cc}
0 & 0 \\
C & 0
\end{array}\right)(\operatorname{deg}+d+1)_{Z_{1}}^{-1} F\left(Z_{1}, Z_{2}\right),
\end{aligned}
$$

and similarly for $(\operatorname{deg}+d)_{Z_{2}}^{-1}$ and $1 \otimes \pi_{r}$.

Lemma 92. Let $F, G \in \mathcal{A}$ and $d \in \mathbb{Z}$. We have the following relations:

$$
\begin{aligned}
& (\operatorname{deg}+d)_{Z_{1}}^{-1}(F * G)=\left((\operatorname{deg}+d)_{Z_{1}}^{-1} F\right) * G, \\
& (\operatorname{deg}+d)_{Z_{2}}^{-1}(F * G)=F *\left((\operatorname{deg}+d)_{Z_{2}}^{-1} G\right), \\
& \left((\operatorname{deg}+d)_{Z_{2}}^{-1} F\right) * G=-F *\left((\operatorname{deg}+3-d)_{Z_{1}}^{-1} G\right) .
\end{aligned}
$$

We define an equivariant map

$$
J=J^{++}-\left(J^{+-}+J^{-+}\right)+J^{--}:\left(\rho_{2}^{\prime}, \mathcal{W}^{\prime}\right) \rightarrow\left(\pi_{l} \otimes \pi_{r}, \mathcal{A}\right)
$$

as follows. Recall the maps $J_{R}$ given by equation (57). By Theorem 65, if $Z_{1}, Z_{2} \in \mathbb{D}_{R}^{+}, J_{R}$ is a $\mathfrak{g l}\left(2, \mathbb{H}_{\mathbb{C}}\right)$-equivariant map $\left(\rho_{2}^{\prime}, \mathcal{W}^{\prime}\right) \rightarrow\left(\pi_{l} \otimes \pi_{r}, \mathcal{V}^{+} \otimes \mathcal{V}^{\prime+}\right)$ independent of the choice of $R>0$; we call this map $J^{++}$. Similarly, if $Z_{1}, Z_{2} \in \mathbb{D}_{R}^{-}, J_{R}$ is a $\mathfrak{g l}\left(2, \mathbb{H}_{\mathbb{C}}\right)$-equivariant map $\left(\rho_{2}^{\prime}, \mathcal{W}^{\prime}\right) \rightarrow\left(\pi_{l} \otimes \pi_{r}, \mathcal{V}^{-} \otimes \mathcal{V}^{\prime-}\right)$ also independent of $R>0$; we call this map $J^{--}$. If $F \in \mathcal{W}^{\prime}$,

$$
J^{+-}(F)=\sum_{\substack{l, m, n \\ l^{\prime}, m^{\prime}, n^{\prime}}} b(F)_{\substack{l, m, n \\ l^{\prime}, m^{\prime}, n^{\prime}}} v_{l, m, n}^{+}\left(Z_{1}\right) \cdot v_{l^{\prime}, m^{\prime}, n^{\prime}}^{-}\left(Z_{2}\right)
$$

where

$$
b(F)_{\substack{l^{\prime}, m^{\prime}, n^{\prime} \\ l, m}}=\frac{12}{\pi^{3} i} \int_{U(2)_{R}} v_{l, m, n}^{\prime-}(W) \cdot F(W) \cdot v_{l^{\prime}, m^{\prime}, n^{\prime}}^{+}(W) d V .
$$

On the one hand, this integral does not depend on $R>0$. On the other hand, by the matrix coefficient expansions of $\frac{(Z-W)^{-1}}{N(Z-W)}$ given in Proposition 26 from [FL1] (see also Proposition 113), for each $R>0$, the series $J^{+-}(F)$ converges to $\left(J_{R} F\right)\left(Z_{1}, Z_{2}\right)$ whenever $Z_{1} \in \mathbb{D}_{R}^{+}$and $Z_{2} \in \mathbb{D}_{R}^{-}$. Similarly,

$$
J^{-+}(F)=\sum_{\substack{l, m, n \\ l, m, m^{\prime}, n^{\prime}}} c(F)_{\substack{l, m, n \\ l^{\prime}, m^{\prime}, n^{\prime}}} v_{l, m, n}^{-}\left(Z_{1}\right) \cdot v_{l^{\prime}, m^{\prime}, n^{\prime}}^{+}\left(Z_{2}\right),
$$

where

$$
c(F)_{\substack{l, m, n \\ l^{\prime}, m^{\prime}, n^{\prime}}}^{l,}=\frac{12}{\pi^{3} i} \int_{U(2)_{R}} v_{l, m, n}^{\prime+}(W) \cdot F(W) \cdot v_{l^{\prime}, m^{\prime}, n^{\prime}}^{-}(W) d V .
$$

This integral is independent of $R>0$ and, for each $R>0$, the series $J^{-+}(F)$ converges to $\left(J_{R} F\right)\left(Z_{1}, Z_{2}\right)$ whenever $Z_{1} \in \mathbb{D}_{R}^{-}$and $Z_{2} \in \mathbb{D}_{R}^{+}$.

Lemma 93. We have:

$$
J\left(N(Z)^{-1} \cdot Z\right)=24 \cdot 1_{\mathcal{A}}
$$

Proof. The result follows immediately from Proposition [66 and expression (80). 
Choose a generator

$$
N(Z)^{-1} \cdot \tilde{H}_{0,0,0}(Z)=N(Z)^{-1} \cdot\left(\begin{array}{ll}
1 & 0 \\
0 & 0
\end{array}\right) \quad \in \mathcal{Q}^{\prime 0} .
$$

We conclude this subsection with an analogue of Proposition 71 ;

Proposition 94. We have:

$$
J\left(N(W)^{-1} \cdot \tilde{H}_{0,0,0}(W)\right)=24\left(\partial_{11}\right)_{Z_{1}}(\operatorname{deg}+1)_{Z_{1}}^{-1} 1_{\mathcal{A}}=24\left(\partial_{11}\right)_{Z_{2}}(\operatorname{deg}+1)_{Z_{2}}^{-1} 1_{\mathcal{A}} .
$$

In particular, for any $G \in \mathcal{A}$,

$$
\begin{aligned}
& J\left(N(W)^{-1} \cdot \tilde{H}_{0,0,0}(W)\right) * G=24\left(\partial_{11}\right)_{Z_{1}}(\operatorname{deg}+1)_{Z_{1}}^{-1} G\left(Z_{1}, Z_{2}\right), \\
& G * J\left(N(W)^{-1} \cdot \tilde{H}_{0,0,0}(W)\right)=24\left(\partial_{11}\right)_{Z_{2}}(\operatorname{deg}+1)_{Z_{2}}^{-1} G\left(Z_{1}, Z_{2}\right) .
\end{aligned}
$$

Proof. We start by computing $J^{+-}$of $N(W)^{-1} \cdot \tilde{H}_{0,0,0}(W)$ :

$$
J^{+-}\left(N(W)^{-1} \cdot \tilde{H}_{0,0,0}(W)\right)\left(Z_{1}, Z_{2}\right)=\sum_{\substack{l, m, n \\ l^{\prime}, m^{\prime}, n^{\prime}}} b(F)_{\substack{l, m, n \\ l^{\prime}, m^{\prime}, n^{\prime}}} v_{l, m, n}^{+}\left(Z_{1}\right) \cdot v_{l^{\prime}, m^{\prime}, n^{\prime}}^{\prime-}\left(Z_{2}\right),
$$

where

$$
\begin{aligned}
& \frac{\pi^{3} i}{12} \cdot b(F)_{\substack{l, m, n \\
l^{\prime}, m^{\prime}, n^{\prime}}}=\int_{U(2)_{R}} v_{l, m, n}^{\prime-}(W) \cdot N(W)^{-1} \cdot\left(\begin{array}{cc}
1 & 0 \\
0 & 0
\end{array}\right) \cdot v_{l^{\prime}, m^{\prime}, n^{\prime}}^{+}(W) d V \\
& =\int_{U(2)_{R}}\left(t_{t_{\underline{n-\frac{1}{2}}}^{l+\frac{1}{2}}}\left(W^{-1}\right), t_{m \underline{n+\frac{1}{2}}}^{l+\frac{1}{2}}\left(W^{-1}\right)\right) \cdot N(W)^{-2} \cdot\left(\begin{array}{ll}
1 & 0 \\
0 & 0
\end{array}\right) \cdot\left(\begin{array}{c}
\left(l^{\prime}-m^{\prime}+\frac{1}{2}\right) t_{n^{\prime}}^{l^{\prime}} \\
\left(l^{\prime}+m^{\prime}+\frac{1}{2}\right) t_{n^{\prime}+\frac{1}{2}}^{l^{\prime}}
\end{array}\right. \\
& =\left(l^{\prime}-m^{\prime}+\frac{1}{2}\right) \int_{U(2)_{R}} N(W)^{-2} \cdot t_{m \underline{m-\frac{1}{2}}}^{l+\frac{1}{2}}\left(W^{-1}\right) \cdot t_{n^{\prime} \underline{m^{\prime}+\frac{1}{2}}}^{l^{\prime}}(W) d V .
\end{aligned}
$$

By the orthogonality relations (19) in [FL3] this coefficient is zero unless $l+1 / 2=l^{\prime}, m=$ $m^{\prime}+1 / 2$ and $n-1 / 2=n^{\prime}$. So, let us assume that this is the case. Using Lemma 22 from [FL1], we obtain:

$$
\begin{aligned}
& J^{+-}\left(N(W)^{-1} \cdot \tilde{H}_{0,0,0}(W)\right)\left(Z_{1}, Z_{2}\right) \\
& =\frac{-24}{N\left(Z_{2}\right)} \sum_{l, m, n} \frac{l-m+\frac{3}{2}}{2 l+2} \cdot\left(\begin{array}{c}
\left(l-m+\frac{1}{2}\right) t_{n+\frac{m}{2}}^{l}\left(Z_{1}\right) \\
\left(l+m+\frac{1}{2}\right) t_{n \underline{m-\frac{1}{2}}}^{l}\left(Z_{1}\right)
\end{array}\right) \cdot\left(t_{m-\frac{1}{2} \underline{n-1}}^{l+1}\left(Z_{2}^{-1}\right), t_{m-\frac{1}{2} \underline{\underline{n}}}^{l+1}\left(Z_{2}^{-1}\right)\right) \\
& =-24 \sum_{l, m, n} \frac{\left(\partial_{11}\right)_{Z_{1}}}{2 l+2} \cdot\left(\begin{array}{c}
\left(l-m+\frac{3}{2}\right) t_{n-\frac{1}{2} \underline{m}}^{l+\frac{1}{2}}\left(Z_{1}\right) \\
\left(l+m+\frac{1}{2}\right) t_{n-\frac{1}{2} \underline{m-1}}^{l+\frac{1}{2}}\left(Z_{1}\right)
\end{array}\right) \cdot N\left(Z_{2}\right)^{-1} \cdot\left(t_{m-\frac{1}{2} \underline{n-1}}^{l+1}\left(Z_{2}^{-1}\right), t_{m-\frac{1}{2} \underline{\underline{n}}}^{l+1}\left(Z_{2}^{-1}\right)\right) .
\end{aligned}
$$

Alternatively, using (37), we can rewrite $J^{+-}\left(N(W)^{-1} \cdot \tilde{H}_{0,0,0}(W)\right)$ as

$$
\begin{aligned}
J^{+-}\left(N(W)^{-1} \cdot \tilde{H}_{0,0,0}(W)\right)\left(Z_{1}, Z_{2}\right) & \\
& =24 \sum_{l, m, n} \frac{\left(\partial_{11}\right) Z_{2}}{2 l+2} \cdot\left(\begin{array}{c}
\left(l-m+\frac{1}{2}\right) t_{n+\frac{1}{2}}^{l}\left(Z_{1}\right) \\
\left(l+m+\frac{1}{2}\right) t_{n \underline{m-\frac{1}{2}}}^{l}\left(Z_{1}\right)
\end{array}\right) \cdot N\left(Z_{2}\right)^{-1} \cdot\left(t_{m_{\underline{n-\frac{1}{2}}}^{l+\frac{1}{2}}}\left(Z_{2}^{-1}\right), t_{m \underline{m+\frac{1}{2}}}^{l+\frac{1}{2}}\left(Z_{2}^{-1}\right)\right) .
\end{aligned}
$$

Next, we find $J^{-+}$of $N(W)^{-1} \cdot \tilde{H}_{0,0,0}(W)$ :

$$
J_{R}\left(N(W)^{-1} \cdot \tilde{H}_{0,0,0}(W)\right)\left(Z_{1}, Z_{2}\right)=\sum_{\substack{l, m, n \\ l^{\prime}, m^{\prime}, n^{\prime}}} c(F)_{\substack{l, m, n \\ l^{\prime}, m^{\prime}, n^{\prime}}} v_{l, m, n}^{-}\left(Z_{1}\right) \cdot v_{l^{\prime}, m^{\prime}, n^{\prime}}^{++}\left(Z_{2}\right),
$$


where

$$
\begin{aligned}
& \frac{\pi^{3} i}{12} \cdot c(F)_{\substack{l, m, n \\
l^{\prime}, m^{\prime}, n^{\prime}}}=\int_{U(2)_{R}} v_{l, m, n}^{++}(W) \cdot N(W)^{-1} \cdot\left(\begin{array}{cc}
1 & 0 \\
0 & 0
\end{array}\right) \cdot v_{l^{\prime}, m^{\prime}, n^{\prime}}^{-}(W) d V \\
& =\int_{U(2)_{R}}\left(t_{m+\frac{1}{2} \underline{n}}^{l-\frac{1}{2}}(W), t_{m-\frac{1}{2} \underline{n}}^{l-\frac{1}{2}}(W)\right) \cdot N(W)^{-2} \cdot\left(\begin{array}{cc}
1 & 0 \\
0 & 0
\end{array}\right) \cdot\left(\begin{array}{c}
\left(l^{\prime}-n^{\prime}+\frac{1}{2}\right) t_{n^{\prime}-\frac{1}{2} \underline{\underline{m}}^{\prime}}^{l^{\prime}} \\
\left(l^{\prime}+n^{\prime}+\frac{1}{2}\right) t_{n^{\prime}+\frac{1}{2} \underline{\underline{m}^{\prime}}}\left(W^{-1}\right)
\end{array}\right) d V \\
& =\left(l^{\prime}-n^{\prime}+\frac{1}{2}\right) \int_{U(2)_{R}} N(W)^{-2} \cdot t_{m+\frac{1}{2} \underline{n}}^{l-\frac{1}{2}}(W) \cdot t_{n^{\prime}-\frac{1}{2}{\underline{m^{\prime}}}^{\prime}}^{l^{\prime}}\left(W^{-1}\right) d V .
\end{aligned}
$$

By the orthogonality relations (19) in [FL3] this coefficient is zero unless $l-1 / 2=l^{\prime}, m+1 / 2=$ $m^{\prime}$ and $n=n^{\prime}-1 / 2$. So, let us assume that this is the case. Using (37), we obtain:

$$
\begin{aligned}
& J^{-+}\left(N(W)^{-1} \cdot \tilde{H}_{0,0,0}(W)\right)\left(Z_{1}, Z_{2}\right) \\
& =-24 \sum_{\substack{l, m, n \\
l \geq 1}} \frac{l-n-\frac{1}{2}}{2 l \cdot N\left(Z_{1}\right)}\left(\begin{array}{c}
\left(l-n+\frac{1}{2}\right) t_{n-\frac{1}{2} \underline{m}}^{l} \\
\left(l+n+\frac{1}{2}\right) t_{n+\frac{1}{2} \underline{m}}^{l}\left(Z_{1}^{-1}\right)
\end{array}\right) \cdot\left(t_{m+1}^{l-1} \underline{n+\frac{1}{2}}\left(Z_{2}\right), t_{{ }_{m}^{l-1} \underline{n+\frac{1}{2}}}^{l-1}\left(Z_{2}\right)\right) \\
& =\frac{24}{N\left(Z_{1}\right)} \sum_{\substack{l, m, n \\
l \geq 1}} \frac{\left(\partial_{11}\right) Z_{1}}{2 l}\left(\begin{array}{c}
\left(l-n-\frac{1}{2}\right) t_{n}^{l-\frac{1}{2}}\left(\begin{array}{c}
n+\frac{1}{2} \\
\left(l+n+\frac{1}{2}\right) t_{n+1}^{l-\frac{1}{2}}\left(Z_{1+\frac{1}{2}}^{-1}\right.
\end{array}\left(Z_{1}^{-1}\right)\right.
\end{array}\right) \cdot\left(t_{m+1 \underline{n+\frac{1}{2}}}^{l-1}\left(Z_{2}\right), t_{m \underline{m+\frac{1}{2}}}^{l-1}\left(Z_{2}\right)\right) .
\end{aligned}
$$

Alternatively, using Lemma 22 from [FL1], we can rewrite $J^{-+}\left(N(W)^{-1} \cdot \tilde{H}_{0,0,0}(W)\right)$ as

$$
\begin{aligned}
& J^{-+}\left(N(W)^{-1} \cdot \tilde{H}_{0,0,0}(W)\right)\left(Z_{1}, Z_{2}\right) \\
& \quad=\frac{-24}{N\left(Z_{1}\right)} \sum_{\substack{l, m, n \\
l \geq 1}} \frac{\left(\partial_{11}\right)_{2}}{2 l}\left(\begin{array}{c}
\left(l-n+\frac{1}{2}\right) t_{n-\frac{1}{2} \underline{m}}^{l}\left(Z_{1}^{-1}\right) \\
\left(l+n+\frac{1}{2}\right) t_{n+\frac{1}{2} \underline{m}}^{l}\left(Z_{1}^{-1}\right)
\end{array}\right) \cdot\left(t_{m+\frac{1}{2} \underline{\underline{n}}}^{l-\frac{1}{2}}\left(Z_{2}\right), t_{m-\frac{1}{2} \underline{\underline{n}}}^{l-\frac{1}{2}}\left(Z_{2}\right)\right) .
\end{aligned}
$$

By Theorem 65,

$$
J^{++}\left(N(W)^{-1} \cdot \tilde{H}_{0,0,0}(W)\right)=J^{--}\left(N(W)^{-1} \cdot \tilde{H}_{0,0,0}(W)\right)=0 .
$$

Thus,

$$
\begin{gathered}
J\left(N(W)^{-1} \cdot \tilde{H}_{0,0,0}(W)\right)\left(Z_{1}, Z_{2}\right)=-\left(J^{+-}+J^{-+}\right)\left(N(W)^{-1} \cdot \tilde{H}_{0,0,0}(W)\right)\left(Z_{1}, Z_{2}\right) \\
=24\left(\partial_{11}\right)_{Z_{1}}(\operatorname{deg}+1)_{Z_{1}}^{-1}\left(\sum_{l, m, n} v_{l, m, n}^{+}\left(Z_{1}\right) \cdot v_{l, m, n}^{\prime-}\left(Z_{2}\right)+\sum_{l, m, n} v_{l, m, n}^{-}\left(Z_{1}\right) \cdot v_{l, m, n}^{\prime+}\left(Z_{2}\right)\right) \\
=24\left(\partial_{11}\right)_{Z_{2}}(\operatorname{deg}+1)_{Z_{2}}^{-1}\left(\sum_{l, m, n} v_{l, m, n}^{+}\left(Z_{1}\right) \cdot v_{l, m, n}^{\prime-}\left(Z_{2}\right)+\sum_{l, m, n} v_{l, m, n}^{-}\left(Z_{1}\right) \cdot v_{l, m, n}^{\prime+}\left(Z_{2}\right)\right) .
\end{gathered}
$$

Then the result follows from (80) and Lemmas 91, 92.

Remark 95. The same argument shows that, for $A \in \mathbb{H}_{\mathbb{C}}$,

$$
J\left(N(W)^{-1} \cdot A\right)=24 \operatorname{Tr}(A \partial)_{Z_{1}}(\operatorname{deg}+1)_{Z_{1}}^{-1} 1_{\mathcal{A}}=24 \operatorname{Tr}(A \partial)_{Z_{2}}(\operatorname{deg}+1)_{Z_{2}}^{-1} 1_{\mathcal{A}} .
$$

In particular,

$$
J\left(N(W)^{-1}\right)=24\left(\partial_{11}+\partial_{22}\right)_{Z_{1}}(\operatorname{deg}+1)_{Z_{1}}^{-1} 1_{\mathcal{A}}=24\left(\partial_{11}+\partial_{22}\right)_{Z_{2}}(\operatorname{deg}+1)_{Z_{2}}^{-1} 1_{\mathcal{A}}
$$




\subsection{Algebra of Quaternionic Functions}

In this subsection we give $\left(\rho_{2}^{\prime}, \mathcal{W}^{\prime} / \operatorname{ker} J\right)$ the structure of a $\mathfrak{g l}\left(2, \mathbb{H}_{\mathbb{C}}\right)$-invariant algebra. Many steps are proved by reduction to the already developed scalar case of $\left(\rho_{1}, \mathscr{W}\right)$.

Definition 96. Let $\mathcal{A}^{\omega}$ denote the subspace of $\mathcal{A}$ generated by $\mathcal{V} \otimes \mathcal{V}^{\prime}, J\left(\mathcal{W}^{\prime}\right)$, application of operators $(\operatorname{deg}+d)_{Z_{1}}^{-1}$ and $(\operatorname{deg}+d)_{Z_{2}}^{-1}, d \in \mathbb{Z}$, as well as actions $\pi_{l} \otimes 1$ and $1 \otimes \pi_{r}$ of $\mathfrak{g l}\left(2, \mathbb{H}_{\mathbb{C}}\right)$.

Thus, by definition, $\mathcal{A}^{\omega}$ is invariant under the $\pi_{l} \otimes \pi_{r}$ action of $\mathfrak{g l}\left(2, \mathbb{H}_{\mathbb{C}}\right)$. As was done in the case of $\mathcal{H} \mathcal{H}^{\omega}$, we want to reduce the number of generators of $\mathcal{A}^{\omega}$.

Lemma 97. The space $\mathcal{A}^{\omega}$ is generated by $\mathcal{V} \otimes \mathcal{V}^{\prime}$, elements of the type

$$
\begin{gathered}
J\left(N(Z)^{-1}\right), \quad\left(\operatorname{deg}+d_{1}\right)_{Z_{1}}^{-1} J\left(N(Z)^{-1}\right), \quad\left(\operatorname{deg}+d_{1}\right)_{Z_{2}}^{-1} J\left(N(Z)^{-1}\right), \\
\left(\operatorname{deg}+d_{2}\right)_{Z_{1}}^{-1}\left(\operatorname{deg}+d_{1}\right)_{Z_{1}}^{-1} J\left(N(Z)^{-1}\right), \quad\left(\operatorname{deg}+d_{2}\right)_{Z_{1}}^{-1}\left(\operatorname{deg}+d_{1}\right)_{Z_{2}}^{-1} J\left(N(Z)^{-1}\right), \\
\left(\operatorname{deg}+d_{2}\right)_{Z_{2}}^{-1}\left(\operatorname{deg}+d_{1}\right)_{Z_{2}}^{-1} J\left(N(Z)^{-1}\right), \quad\left(\operatorname{deg}+d_{3}\right)_{Z_{1}}^{-1}\left(\operatorname{deg}+d_{2}\right)_{Z_{1}}^{-1}\left(\operatorname{deg}+d_{1}\right)_{Z_{1}}^{-1} J\left(N(Z)^{-1}\right), \ldots
\end{gathered}
$$

as well as actions $\pi_{l} \otimes 1$ and $1 \otimes \pi_{r}$ of $\mathfrak{g l}\left(2, \mathbb{H}_{\mathbb{C}}\right)$.

Proof. By Theorem 65 and Proposition 66, $J\left(\mathcal{W}^{\prime}\right) \simeq \mathcal{W}^{\prime} /$ ker $J$ has four irreducible components:

$$
\mathcal{Q}^{\prime+}, \quad \mathcal{Q}^{\prime 0}, \quad \mathcal{Q}^{\prime-}
$$

and the trivial one-dimensional representation generated by $N(Z)^{-1} \cdot Z$. Since $\left(\rho_{2}^{\prime}, \mathcal{W}^{\prime}\right)$ and $\left(\rho_{2}, \mathcal{W}\right)$ are linear dual to each other, by Lemma 59, the one-dimensional representation appears as a subrepresentation in $J\left(\mathcal{W}^{\prime}\right)$ and the irreducible component $\mathcal{Q}^{\prime 0}$ only as a subquotient. By Theorem 65] again,

$$
J\left(\mathcal{Q}^{\prime+} \oplus \mathcal{Q}^{\prime-}\right) \subset \mathcal{V} \otimes \mathcal{V}^{\prime}
$$

On the other hand, $N(Z)^{-1}$ generates both $\mathcal{Q}^{\prime 0}$ and the one-dimensional component. Then the proof proceeds the same way as that of Lemma 73 .

Proposition 98. The space $\mathcal{A}^{\omega}$ is closed under the convolution operation: if $F, G \in \mathcal{A}^{\omega}$, then $F * G$ also lies in $\mathcal{A}^{\omega}$.

Proof. The proof is essentially the same as that of Proposition 74.

As was done in the case of $\mathcal{H} \mathcal{H}^{\omega}$, we want to realize elements of $\mathcal{A}^{\omega}$ as analytic functions. Recall open subset $\Omega$ of $\mathbb{H}_{\mathbb{C}}^{\times} \times \mathbb{H}_{\mathbb{C}}^{\times}$introduced in Subsection 7.2 . There is a natural map $\omega$ from

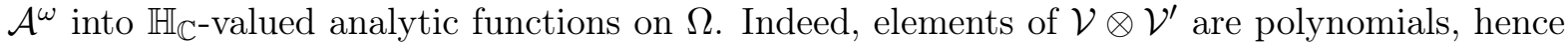
can be treated as analytic functions on $\Omega$. On the other hand, by (59),

$$
J\left(N(Z)^{-1}\right)=24 \vec{\partial}_{Z_{1}} I\left(N(Z)^{-1}\right) \overleftarrow{\partial}_{Z_{2}}
$$

is an analytic function on $\Omega$ also. Then operators $(\operatorname{deg}+d)_{Z_{1}}^{-1}$ and $(\operatorname{deg}+d)_{Z_{2}}^{-1}, d \in \mathbb{Z}$, as well as actions $\pi_{l} \otimes 1$ and $1 \otimes \pi_{r}$ of $\mathfrak{g l}\left(2, \mathbb{H}_{\mathbb{C}}\right)$ preserve analyticity of functions. Unlike the case of $\mathcal{H H}^{\omega}$, this map $\omega$ has a non-trivial kernel: $\omega\left(1_{\mathcal{A}}\right)=0$, by Proposition 66. We denote the composition $\omega \circ J$ by $\tilde{J}$ and the space of analytic functions on $\Omega$ that are in the image of $\omega$ by $\tilde{\mathcal{A}}^{\omega}$. Note that $\operatorname{ker} \tilde{J}=\operatorname{ker} \mathrm{Mx}$ and

$$
\tilde{J}(F)=24 \vec{\partial}_{Z_{1}} I(F) \overleftarrow{\partial}_{Z_{2}}, \quad F \in \mathcal{W}^{\prime}
$$

Each $F \in \tilde{\mathcal{A}}^{\omega}$ is left regular with respect to $Z_{1}$ and right regular with respect to $Z_{2}$ :

$$
\vec{\nabla}_{Z_{1}} F\left(Z_{1}, Z_{2}\right)=0=F\left(Z_{1}, Z_{2}\right) \overleftarrow{\nabla}_{Z_{2}}
$$


Since

$$
\mathcal{V} \otimes \mathcal{V}^{\prime} \subset \tilde{\mathcal{A}}^{\omega}
$$

we can think of $\tilde{\mathcal{A}}^{\omega}$ as a completion of $\mathcal{V} \otimes \mathcal{V}^{\prime}$. to $\tilde{\mathcal{A}}^{\omega}$.

Recall open subsets $\Lambda^{+}, \Lambda^{-}$of $\mathbb{H}_{\mathbb{C}}^{\times} \times \mathbb{H}_{\mathbb{C}}^{\times}$introduced in Subsection [7.2. Definition 76 extends

Definition 99. We call a function $F \in \tilde{\mathcal{A}}^{\omega}$ extendable if, for each $Z_{0} \in \mathbb{H}_{\mathbb{C}}^{\times}$, there exists an

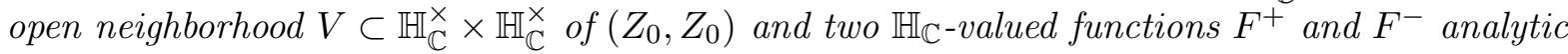
on $V$ such that $F=F^{+}$for all points in $\Lambda^{+} \cap V$ and $F=F^{-}$for all points in $\Lambda^{-} \cap V$.

Clearly, elements of $\mathcal{V} \otimes \mathcal{V}^{\prime}$ are extendable.

Lemma 100. The extendable functions in $\tilde{\mathcal{A}}^{\omega}$ form a subspace that is invariant under the actions $\pi_{l} \otimes 1,1 \otimes \pi_{r}$ and $\pi_{l} \otimes \pi_{r}$ of $\mathfrak{g l}\left(2, \mathbb{H}_{\mathbb{C}}\right)$.

Remark 101. We expect all functions in $\tilde{\mathcal{A}}^{\omega}$ to be extendable.

Let $F \in \tilde{\mathcal{A}}^{\omega}$ be an extendable function, define two analytic functions on $\mathbb{H}_{\mathbb{C}}^{\times}$:

$$
\begin{aligned}
& \left(\operatorname{Diag}^{+} F\right)(Z)=\lim _{\substack{Z_{1}, Z_{2} \rightarrow Z \\
\left(Z_{1}, Z_{2}\right) \in \Lambda^{+}}} F\left(Z_{1}, Z_{2}\right) \quad \text { and } \\
& \left(\operatorname{Diag}^{-} F\right)(Z)=\lim _{\substack{Z_{1}, Z_{2} \rightarrow Z \\
\left(Z_{1}, Z_{2}\right) \in \Lambda^{-}}} F\left(Z_{1}, Z_{2}\right) .
\end{aligned}
$$

As in the scalar case, applying $\operatorname{Diag}^{+} F$ and $\operatorname{Diag}^{-} F$ may yield different results; functions $\operatorname{Diag}^{+} F$ and $\operatorname{Diag}^{-} F$ need not be elements of $\mathcal{W}$ because they may not be polynomials on $\mathbb{H}_{\mathbb{C}}^{\times}$. Nevertheless, the operators Diag ${ }^{+}$and Diag ${ }^{-}$intertwine the $\mathfrak{g l}\left(2, \mathbb{H}_{\mathbb{C}}\right)$-actions $\pi_{l} \otimes \pi_{r}$ on

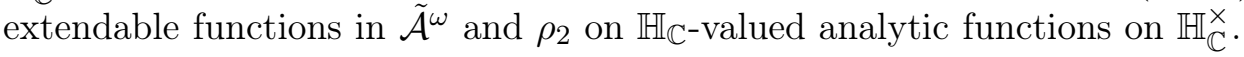

Lemma 102. If $F\left(Z_{1}, Z_{2}\right) \in \mathcal{V} \otimes \mathcal{V}^{\prime}$, then

$$
\operatorname{Diag}^{+} F=\operatorname{Diag}^{-} F \quad \in \mathcal{Q}^{+} \oplus \mathcal{Q}^{0} \oplus \mathcal{Q}^{-} .
$$

Proof. Note that, when restricted to $\mathcal{V} \otimes \mathcal{V}$, both $\mathrm{Diag}^{+}$and $\mathrm{Diag}^{-}$reduce to the multiplication map Mult $: \mathcal{V} \otimes \mathcal{V}^{\prime} \rightarrow \mathcal{W}$, then the result follows from Proposition 89.

Lemma 103. For each $F \in \mathcal{W}^{\prime}, \tilde{J}(F)$ is extendable and can be written as a finite linear

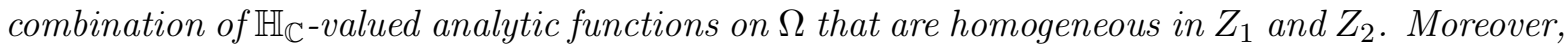

$$
\operatorname{Diag}^{+} \tilde{J}(F)=\operatorname{Diag}^{-} \tilde{J}(F)=\operatorname{Mx} F \quad \in \mathcal{Q}^{+} \oplus \mathcal{Q}^{0} \oplus \mathcal{Q}^{-} .
$$

Proof. The first part follows from Lemma 79 and equation (81). The second part follows from Theorems 65 and 67.

Lemma 104. For each $d \in \mathbb{Z},(\operatorname{deg}+d)_{Z_{1}}^{-1} \tilde{J}\left(N(Z)^{-1}\right)$ and $(\operatorname{deg}+d)_{Z_{2}}^{-1} \tilde{J}\left(N(Z)^{-1}\right)$ are extendable.

Proof. The result follows from Lemma 82 and equation (81).

Theorem 105. Let $F, G \in \mathcal{W}^{\prime}$, then $\omega(J(F) * J(G)) \in \tilde{\mathcal{A}}^{\omega}$ is extendable and

$$
\operatorname{Diag}^{+}(\omega(J(F) * J(G))), \quad \operatorname{Diag}^{-}(\omega(J(F) * J(G))) \quad \in \mathcal{Q}^{+} \oplus \mathcal{Q}^{0} \oplus \mathcal{Q}^{-} .
$$


Proof. Recall that $\mathcal{W}^{\prime} / \operatorname{ker} J$ has four irreducible components:

$$
\mathcal{Q}^{\prime+}, \quad \mathcal{Q}^{\prime 0}, \quad \mathcal{Q}^{\prime-}
$$

and the trivial one-dimensional subrepresentation generated by $N(Z)^{-1} \cdot Z$. If $F$ or $G \in \mathcal{Q}^{\prime-} \oplus$ $\mathcal{Q}^{\prime+}$, then, by Theorem 65, $J(F)$ or $J(G) \in \mathcal{V} \otimes \mathcal{V}^{\prime}$, and the result follows from Lemmas 90 , 102 ,

If $F$ or $G$ is proportional to $N(Z)^{-1} \cdot Z$, then, by Lemma 93, $J(F) * J(G)$ is proportional to $J(F)$ or $J(G)$, and the result follows from Lemma 103 .

It remains to consider the case $F, G \in \mathcal{Q}^{\prime 0}$. Since $\mathcal{Q}^{\prime 0}$ is irreducible and can be generated by either $N(Z)^{-1}$ or $N(Z)^{-1} \cdot \tilde{H}_{0,0,0}(Z)$, Proposition 94 and Lemmas 91,100 and 104 imply that $\omega(J(F) * J(G))$ is extendable. We still need to prove property (82). As usual, let $K=U(2) \times U(2)$ and observe that $F, G \in \mathcal{W}^{\prime}$ are $K$-finite. Since all operations involved intertwine the actions of $\mathfrak{g l}\left(2, \mathbb{H}_{\mathbb{C}}\right)$, analytic functions $\operatorname{Diag}^{+}(\omega(J(F) * J(G)))$ and $\operatorname{Diag}^{-}(\omega(J(F) * J(G)))$ on $\mathbb{H}_{\mathbb{C}}^{\times}$are $K$-finite as well. By Proposition $60,\left(\rho_{2}^{\prime}, \mathcal{W}^{\prime}\right)$ and its dual $\left(\rho_{2}, \mathcal{W}\right)$ are admissible $(\mathfrak{g}, K)$-modules. Since the elements of $\mathcal{W}$ are dense in the space of all analytic functions on $\mathbb{H}_{\mathbb{C}}^{\times}$, it follows that

$$
\operatorname{Diag}^{+}(\omega(J(F) * J(G))), \quad \operatorname{Diag}^{-}(\omega(J(F) * J(G))) \quad \in \mathcal{W} .
$$

On the other hand, by Proposition 89, $\operatorname{Diag}^{+}(\omega(J(F) * J(G)))$ and $\operatorname{Diag}^{-}(\omega(J(F) * J(G)))$ must be in the closure of $\mathcal{Q}^{+} \oplus \mathcal{Q}^{0} \oplus \mathcal{Q}^{-}$, then (82) follows.

Theorem 105 allows us to define two $\mathfrak{g l}\left(2, \mathbb{H}_{\mathbb{C}}\right)$-invariant multiplication-like operations

$$
\left(\mathcal{W}^{\prime} / \operatorname{ker} J\right) \otimes\left(\mathcal{W}^{\prime} / \operatorname{ker} J\right) \rightarrow \mathcal{W}^{\prime} / \operatorname{ker} \mathrm{Mx}
$$

as follows. Recall $\mathrm{Mx}: \mathcal{W}^{\prime} \rightarrow \mathcal{W}$, its image is $\mathcal{Q}^{+} \oplus \mathcal{Q}^{0} \oplus \mathcal{Q}^{-}$. Let

$$
\mathrm{Mx}^{-1}: \mathcal{Q}^{+} \oplus \mathcal{Q}^{0} \oplus \mathcal{Q}^{-} \rightarrow \mathcal{W}^{\prime} / \operatorname{ker} \mathrm{Mx}
$$

be the inverse isomorphism to $\mathrm{Mx}: \mathcal{W}^{\prime} / \operatorname{ker} \mathrm{Mx} \rightarrow \operatorname{Mx}\left(\mathcal{W}^{\prime}\right) \subset \mathcal{W}$.

Definition 106. Let $F, G \in \mathcal{W}^{\prime} /$ ker $J$, define

$$
\begin{aligned}
& F *^{+} G=\mathrm{Mx}^{-1} \circ \operatorname{Diag}^{+}(\omega(J(F) * J(G))), \\
& F *^{-} G=\mathrm{Mx}^{-1} \circ \operatorname{Diag}^{-}(\omega(J(F) * J(G))) .
\end{aligned}
$$

Remark 107. Note that $\mathcal{W}^{\prime} / \operatorname{ker} \mathrm{Mx}=\mathcal{W}^{\prime} / \operatorname{ker} \tilde{J}$ is the quotient of $\mathcal{W}^{\prime} / \operatorname{ker} J$ by the onedimensional trivial subrepresentation spanned by $N(Z)^{-1} \cdot Z$.

Proposition 108. One can also consider multiplications obtained by taking linear combinations of $F *^{+} G$ and $F *^{-} G$. Thus we obtain a one-parameter family of $\mathfrak{g l}\left(2, \mathbb{H}_{\mathbb{C}}\right)$-equivariant maps

$$
\left(\mathcal{W}^{\prime} / \operatorname{ker} J\right) \otimes\left(\mathcal{W}^{\prime} / \operatorname{ker} J\right) \rightarrow \mathcal{W}^{\prime} / \operatorname{ker} \mathrm{Mx} .
$$

If $F_{1}, \ldots, F_{n} \in \mathcal{W}^{\prime} /$ ker $J$, we expect

$$
\omega\left(J\left(F_{1}\right) * \cdots * J\left(F_{n}\right)\right) \quad \in \tilde{\mathcal{A}}^{\omega}
$$

to be extendable. Once this is established, we can define $\mathfrak{g l}\left(2, \mathbb{H}_{\mathbb{C}}\right)$-invariant $n$-multiplications:

Definition 109. Let $F_{1}, \ldots, F_{n} \in \mathcal{W}^{\prime} / \operatorname{ker} J$, define

$$
\begin{gathered}
\underbrace{\left(\mathcal{W}^{\prime} / \operatorname{ker} J\right) \times \cdots \times\left(\mathcal{W}^{\prime} / \operatorname{ker} J\right)}_{n \text { times }} \rightarrow \mathcal{W}^{\prime} / \operatorname{ker} \mathrm{Mx}: \\
F_{1} *^{+} \ldots *^{+} F_{n}=\mathrm{Mx}^{-1} \circ \operatorname{Diag}^{+}\left(\omega\left(J\left(F_{1}\right) * \cdots * J\left(F_{n}\right)\right)\right), \\
F_{1} *^{-} \cdots *^{-} F_{n}=\mathrm{Mx}^{-1} \circ \operatorname{Diag}^{-}\left(\omega\left(J\left(F_{1}\right) * \cdots * J\left(F_{n}\right)\right)\right) .
\end{gathered}
$$


Remark 110. Once again, $\mathcal{W}^{\prime} / \operatorname{ker} \mathrm{Mx}$ is the quotient of $\mathcal{W}^{\prime} / \operatorname{ker} J$ by the one-dimensional trivial subrepresentation. In a future work we intend to "lift" these operations to genuine multiplications

$$
\underbrace{\left(\mathcal{W}^{\prime} / \operatorname{ker} J\right) \times \cdots \times\left(\mathcal{W}^{\prime} / \operatorname{ker} J\right)}_{n \text { times }} \rightarrow \mathcal{W}^{\prime} / \operatorname{ker} J
$$

Furthermore, we can also consider n-multiplications obtained by taking linear combinations of $F_{1} *^{+} \ldots *^{+} F_{n}$ and $F_{1} *^{-} \cdots *^{-} F_{n}$. Thus we obtain a one-parameter family of $\mathfrak{g l}\left(2, \mathbb{H}_{\mathbb{C}}\right)$ invariant multiplications

$$
\underbrace{\left(\mathcal{W}^{\prime} / \operatorname{ker} J\right) \otimes \cdots \otimes\left(\mathcal{W}^{\prime} / \operatorname{ker} J\right)}_{n \text { times }} \rightarrow \mathcal{W}^{\prime} / \text { ker } J
$$

We conclude this subsection with some thoughts about lifting operations

$$
*^{ \pm}:\left(\mathcal{W}^{\prime} / \operatorname{ker} J\right) \otimes\left(\mathcal{W}^{\prime} / \operatorname{ker} J\right) \rightarrow \mathcal{W}^{\prime} / \operatorname{ker} \mathrm{Mx}
$$

to $\mathfrak{g l}\left(2, \mathbb{H}_{\mathbb{C}}\right)$-invariant multiplication operations on $\mathcal{W}^{\prime} / \operatorname{ker} J$ and properties of these multiplication operations. Recall that the definition of $*^{+}$and $*^{-}$involves a composition of maps factoring through a subspace of $\mathcal{W}$ (Definition 106). Our first observation is that a multiplication operation on $\mathcal{W}^{\prime} /$ ker $J$ cannot be factored through a subspace of $\mathcal{W}$ because there does not exist a $\rho_{2}$-invariant subspace of $\mathcal{W}$ isomorphic to $\mathcal{W}^{\prime} / \operatorname{ker} J$. Indeed, the only $\rho_{2}$-invariant subspace of $\mathcal{W}$ that has the same irreducible components as $\mathcal{W}^{\prime} /$ ker $J$ is the subspace of $\left(\rho_{2}, \mathcal{W}\right)$ generated by $\mathcal{Q}^{+} \oplus \mathcal{Q}^{0} \oplus \mathcal{Q}^{-}$and $N(Z)^{-2} \cdot Z^{+}$. But this subspace is still not isomorphic to $\mathcal{W}^{\prime} / \operatorname{ker} J$ because of Lemma 59.

Next, we assume that a $\mathfrak{g l}\left(2, \mathbb{H}_{\mathbb{C}}\right)$-invariant multiplication operation lifting $*^{+}$or $*^{-}$is defined and try to derive some of its properties. As usual, let $K=U(2) \times U(2)$. Recall that $\mathcal{W}^{\prime} / \operatorname{ker} J$ has four irreducible components:

$$
\mathcal{Q}^{\prime+}, \quad \mathcal{Q}^{\prime 0}, \quad \mathcal{Q}^{\prime-}
$$

and the trivial one-dimensional representation generated by $N(Z)^{-1} \cdot Z$. The one-dimensional representation appears as a subrepresentation in $\mathcal{W}^{\prime} / \operatorname{ker} J$ and the irreducible component $\mathcal{Q}^{\prime 0}$ only as a subquotient. We decompose $\mathcal{W}^{\prime} /$ ker $J$ as a direct sum of $K$-invariant subspaces:

$$
\mathcal{W}^{\prime} / \operatorname{ker} J \simeq \mathbb{C} \oplus \mathcal{Q}^{\prime+} \oplus \mathcal{Q}^{\prime 0} \oplus \mathcal{Q}^{\prime-},
$$

where $\mathbb{C}$ denotes the trivial one-dimensional representation generated by $N(Z)^{-1} \cdot Z$. We emphasize that this direct sum decomposition is not $\mathfrak{g l}\left(2, \mathbb{H}_{\mathbb{C}}\right)$-invariant, since the subspace $\mathcal{Q}^{\prime 0}$ is not $\mathfrak{g l}\left(2, \mathbb{H}_{\mathbb{C}}\right)$-invariant, it is only $K$-invariant. Since

$$
\mathcal{W}^{\prime} / \text { ker } \mathrm{Mx} \simeq \mathcal{Q}^{\prime+} \oplus \mathcal{Q}^{\prime 0} \oplus \mathcal{Q}^{\prime-},
$$

we have a direct sum decomposition of $K$-invariant subspaces

$$
\mathcal{W}^{\prime} / \operatorname{ker} J \simeq \mathbb{C} \oplus\left(\mathcal{W}^{\prime} / \operatorname{ker} \mathrm{Mx}\right),
$$

and we can write elements of $\mathcal{W}^{\prime} / \operatorname{ker} J$ as pairs

$$
(c, w), \quad c \in \mathbb{C}, w \in \mathcal{W}^{\prime} / \operatorname{ker} \mathrm{Mx} .
$$

For example, by Lemma 93, element $N(Z)^{-1} \cdot Z$ corresponds to a pair $(24,0) \in \mathbb{C} \oplus\left(\mathcal{W}^{\prime} /\right.$ ker Mx $)$. Then lifting an operation from

$$
*^{ \pm}:\left(\mathcal{W}^{\prime} / \operatorname{ker} J\right) \otimes\left(\mathcal{W}^{\prime} / \operatorname{ker} J\right) \rightarrow \mathcal{W}^{\prime} / \operatorname{ker} \mathrm{Mx}
$$


to multiplication

$$
\tilde{*}^{ \pm}:\left(\mathcal{W}^{\prime} / \operatorname{ker} J\right) \otimes\left(\mathcal{W}^{\prime} / \operatorname{ker} J\right) \rightarrow \mathcal{W}^{\prime} / \operatorname{ker} J
$$

amounts to specifying a $\mathbb{C}$-valued bilinear pairing

$$
c^{ \pm}\left(F_{1}, F_{2}\right), \quad F_{1}, F_{2} \in \mathcal{W}^{\prime} / \operatorname{ker} J
$$

so that the lift is

$$
F_{1} \tilde{*}^{ \pm} F_{2}=\left(c^{ \pm}\left(F_{1}, F_{2}\right), F_{1} *^{ \pm} F_{2}\right) \quad \in \mathbb{C} \oplus\left(\mathcal{W}^{\prime} / \text { ker Mx }\right) .
$$

If $\tilde{*}^{ \pm}$is $K$-invariant, then so is $c^{ \pm}\left(F_{1}, F_{2}\right)$.

Lemma 111. Suppose that the multiplication operation $\tilde{*}^{ \pm}$on $\mathcal{W}^{\prime} / \operatorname{ker} J$ is $\mathfrak{g l}\left(2, \mathbb{H}_{\mathbb{C}}\right)$-invariant. Then the bilinear pairing $c^{ \pm}\left(F_{1}, F_{2}\right)$ cannot be $\mathfrak{g l}\left(2, \mathbb{H}_{\mathbb{C}}\right)$-invariant.

Proof. First, we spell out the $\mathfrak{g l}\left(2, \mathbb{H}_{\mathbb{C}}\right)$-invariance of $\tilde{*}^{ \pm}$:

$$
\left(\rho_{2}^{\prime}(X) F_{1}\right) \tilde{*}^{ \pm} F_{2}+F_{1} \tilde{*}^{ \pm}\left(\rho_{2}^{\prime}(X) F_{2}\right)=\rho_{2}^{\prime}(X)\left(F_{1} \tilde{*}^{ \pm} F_{2}\right),
$$

for all $F_{1}, F_{2} \in \mathcal{W}^{\prime} / \operatorname{ker} J$ and all $X \in \mathfrak{g l}\left(2, \mathbb{H}_{\mathbb{C}}\right)$. Since $\mathfrak{g l}\left(2, \mathbb{H}_{\mathbb{C}}\right)$ acts on the $\mathbb{C}$-component of (83) trivially,

$$
\rho_{2}^{\prime}(X)\left(F_{1} \tilde{*}^{ \pm} F_{2}\right)=\rho_{2}^{\prime}(X)\left(F_{1} *^{ \pm} F_{2}\right) .
$$

If the bilinear pairing $c^{ \pm}\left(F_{1}, F_{2}\right)$ is $\mathfrak{g l}\left(2, \mathbb{H}_{\mathbb{C}}\right)$-invariant, then the $\mathbb{C}$-component of the left hand side of (84) is zero.

On the other hand, the image of

$$
*^{ \pm}:\left(\mathcal{W}^{\prime} / \operatorname{ker} J\right) \otimes\left(\mathcal{W}^{\prime} / \operatorname{ker} J\right) \rightarrow \mathcal{W}^{\prime} / \operatorname{ker} \mathrm{Mx}
$$

contains $\mathcal{Q}^{\prime 0}$. But $\mathcal{Q}^{\prime 0}$ is not a $\mathfrak{g l}\left(2, \mathbb{H}_{\mathbb{C}}\right)$-invariant subspace of $\mathcal{W}^{\prime} /$ ker $J$ - there exist an $F \in \mathcal{Q}^{\prime 0}$ and an $X \in \mathfrak{g l}\left(2, \mathbb{H}_{\mathbb{C}}\right)$ such that $\rho_{2}^{\prime}(X) F$ has non-zero $\mathbb{C}$-component. Hence there exist elements $F_{1}, F_{2} \in \mathcal{W}^{\prime} /$ ker $J$ and an $X \in \mathfrak{g l}\left(2, \mathbb{H}_{\mathbb{C}}\right)$ such that the $\mathbb{C}$-component of

$$
\rho_{2}^{\prime}(X)\left(F_{1} \tilde{*}^{ \pm} F_{2}\right)=\rho_{2}^{\prime}(X)\left(F_{1} *^{ \pm} F_{2}\right) \quad \in \mathcal{W}^{\prime} / \operatorname{ker} J
$$

is not zero. Substituting such $F_{1}, F_{2}$ and $X$ into (84) produces a contradiction.

Thus, lifting $*^{+}\left(\right.$or $\left.*^{-}\right)$to a $\mathfrak{g l}\left(2, \mathbb{H}_{\mathbb{C}}\right)$-invariant multiplication operation $\tilde{*}^{+}\left(\right.$or $\left.\tilde{*}^{-}\right)$on $\mathcal{W}^{\prime} /$ ker $J$ amounts to specifying a certain $K$-invariant bilinear pairing $c^{+}$(or $c^{-}$) on $\mathcal{W}^{\prime} / \operatorname{ker} J$. But, since this pairing cannot be $\mathfrak{g l}\left(2, \mathbb{H}_{\mathbb{C}}\right)$-invariant, finding such a pairing that would make the resulting multiplication operation $\mathfrak{g l}\left(2, \mathbb{H}_{\mathbb{C}}\right)$-invariant is not trivial.

\subsection{Properties of Multiplications on Quaternionic Algebras}

We know that the quaternionic algebra $\mathcal{W}^{\prime} /$ ker $J$ and its scalar counterpart $\mathscr{T}$ are not associative (Example 86]). On the other hand, in both cases we have indicated how to define $\mathfrak{g l}\left(2, \mathbb{H}_{\mathbb{C}}\right)$ invariant $n$-multiplications (Proposition 87 and Remark 110). It is natural to conjecture that these $n$-multiplications satisfy some sort of relaxed associativity-type relations. There is a wellknown structure of this kind known as an $A_{\infty}$-algebra. There are several types of $A_{\infty}$-algebras, and, in order to formulate our conjecture more explicitly, we recall some basic definitions (see, for example, $[\mathrm{K}]$ for details).

An $A_{\infty}$ algebra over $\mathbb{C}$ is a complex vector space $A$ endowed with maps

$$
\nu_{n}: A^{\otimes n}=\underbrace{A \otimes \cdots \otimes A}_{n \text { times }} \rightarrow A, \quad n=1,2,3, \ldots,
$$


such that, for all $n \geq 1$, we have associativity-type identities of the form

$$
\sum_{a+b+c=n} \nu_{a+1+c} \circ\left(\mathbb{1}^{\otimes a} \otimes \nu_{b} \otimes \mathbb{1}^{\otimes c}\right)=0
$$

as maps from $A^{\otimes n}$ to $A$. For $n=1,2,3$, these identities become

$$
\begin{aligned}
& \nu_{1} \circ \nu_{1}=0 \\
& \nu_{1} \circ \nu_{2}+\nu_{2}\left(\nu_{1} \otimes \mathbb{1}+\mathbb{1} \otimes \nu_{1}\right)=0, \\
& \nu_{1} \circ \nu_{3}+\nu_{2}\left(\nu_{2} \otimes \mathbb{1}+\mathbb{1} \otimes \nu_{2}\right)+\nu_{3}\left(\nu_{1} \otimes \mathbb{1} \otimes \mathbb{1}+\mathbb{1} \otimes \nu_{1} \otimes \mathbb{1}+\mathbb{1} \otimes \mathbb{1} \otimes \nu_{1}\right)=0 .
\end{aligned}
$$

The first identity (86) states that $A$ is a complex, the second (87) means that $\nu_{2}: A \otimes A \rightarrow A$ is a morphism of complexes. Neither of these two assertions seem natural for the quaternionic algebras. However, there is a more general notion of a weak $A_{\infty}$-algebra with an additional map

$$
\nu_{0}: \mathbb{C} \rightarrow A
$$

such that the identities (85) hold after one includes the additional terms with $\nu_{0}$. For example, for $n=1,2$, identities (86)- (87) become

$$
\begin{aligned}
& \nu_{1} \circ \nu_{1}+\nu_{2}\left(\nu_{0} \otimes \mathbb{1}+\mathbb{1} \otimes \nu_{0}\right)=0, \\
& \nu_{1} \circ \nu_{2}+\nu_{2}\left(\nu_{1} \otimes \mathbb{1}+\mathbb{1} \otimes \nu_{1}\right)+\nu_{3}\left(\nu_{0} \otimes \mathbb{1} \otimes \mathbb{1}+\mathbb{1} \otimes \nu_{0} \otimes \mathbb{1}+\mathbb{1} \otimes \mathbb{1} \otimes \nu_{0}\right)=0 .
\end{aligned}
$$

Identity (89) is satisfied when $\nu_{1}$ is the identity map on $A$ and

$$
\nu_{2}\left(\nu_{0}(1), a\right)+\nu_{2}\left(a, \nu_{0}(1)\right)=-a, \quad \forall a \in A .
$$

Thus we can define multiplication on $A$ by setting

$$
\mu_{2}=-\frac{1}{2} \nu_{2}
$$

then (91) will be satisfied if $\nu_{0}(1)$ is the (left and right) unit for the multiplication operation $\mu_{2}$. Similarly, choosing appropriate coefficients, we can relate $\nu_{n}$ with $n$-multiplications $\mu_{n}$ for our quaternionic algebras.

We also expect that our $n$-multiplications $\mu_{n}$ satisfy a certain cyclic symmetry. Namely, there is a $\mathfrak{g l}\left(2, \mathbb{H}_{\mathbb{C}}\right)$-invariant bilinear product $\langle\cdot, \cdot\rangle$ such that

$$
\left\langle\mu_{n}\left(a_{1}, \ldots, a_{n}\right), a_{n+1}\right\rangle=\left\langle a_{1}, \mu_{n}\left(a_{2}, \ldots, a_{n+1}\right)\right\rangle, \quad \forall a_{1}, \ldots, a_{n+1} \in A .
$$

Those $A_{\infty}$-algebras that satisfy the additional property (92) are called cyclic $A_{\infty}$-algebras, in our case weak cyclic $A_{\infty}$-algebras.

In the case of quaternionic algebras, the cyclic symmetry of $n$-multiplications should follow from the conjectural identification of the paired $n$-products as in (92) with variants of the $(n+1)$-photon diagrams (Figure 11). In fact, our map

$$
J: \mathcal{W}^{\prime} \rightarrow \mathcal{A}=\text { completion of } \mathcal{V} \otimes \mathcal{V}^{\prime}
$$

corresponds to the vertices in this diagram, with spaces $\mathcal{V}$ and $\mathcal{V}^{\prime}$ identified with the solutions of the massless Dirac equations. Note that we are considering the chiral case of $n$-photon diagrams; in the non-chiral case all the diagrams with odd number of vertices cancel out and yield a zero result. Also, the Maxwell equations - which define the classical photon space and play an important role in the quantum theory - are crucial to our definition of algebra of quaternionic 
functions. One can ask then, what might be the physical meaning of the associativity-type identities (85)-(90). It turns out that, despite the long history of four-dimensional quantum field theory, only recently there were discovered certain quadratic relations, first, in quantum YangMills theory [BCFW]. These relations were later extended to one-loop multi-photon diagrams in QED (see [BBBV] and references therein), and they might provide a source of associativity in quaternionic algebra. Note that there is also a scalar counterpart of QED, which has an analogous structure, including the quadratic identities. This scalar version of QED is expected to match our scalar quaternionic algebra, including the associativity-type identities.

To make the identification of our quaternionic constructions with physics more transparent, we can "translate" all our definitions related to quaternionic algebra from the quaternionic space into the Minkowski space using the Cayley transform, as we did in [FL1]. In [FL1], the switch to the Minkowski space via the Cayley transform was crucial for demonstrating unitarity of the spaces of (left and right) regular functions. The unitarity of $\left(\rho_{1}, \mathscr{W}\right)$ was shown in Proposition 9 of [FL3]. The same methods and motivations also apply to the spaces of (left and right) doubly regular functions and to the underlying space of quaternionic algebra $\mathcal{W}^{\prime} /$ ker $J$ factored by the one dimensional subrepresentation. On the physics side, the unitarity is fundamental in four-dimensional quantum field theory, in particular in QED. Minkowski space realization of our current results also suggests an interesting problem of finding of physical meaning of our present results in quaternionic analysis, including the decomposition of spaces $\mathcal{W}$ and $\mathcal{W}^{\prime}$ into irreducible components, the role of the one-dimensional representation in vacuum polarization and the meaning of the quaternionic algebras.

Comparing the structures of quaternionic analysis with those of four-dimensional quantum field theory will be beneficial to both disciplines. On the one hand, various techniques of calculations and regularizations of Feynman integrals should apply to different constructions of quaternionic analysis, including the quaternionic algebras. On the other hand, our clear conceptual program of quaternionic analysis developed along the lines of well-established complex analysis and carried out to a new step in this paper might eventually provide a purely mathematical foundation of the vast number of scattered calculations, curious identities and remarkable cancellations in the still mysterious subject of four-dimensional quantum physics.

\section{Appendix: Comments about [FL1] and [FL3]}

We would like to add some comments about [FL1] and [FL3] that are relevant to the present article.

\subsection{Comments about [FL1]}

Lemma 17 describing the Lie algebra actions $\pi_{l}^{0}$ and $\pi_{r}^{0}$ of $\mathfrak{g l}(2, \mathbb{H})$ on the space of harmonic functions should state

$$
\pi_{l}^{0}\left(\begin{array}{cc}
0 & 0 \\
C & 0
\end{array}\right)=\pi_{r}^{0}\left(\begin{array}{cc}
0 & 0 \\
C & 0
\end{array}\right): \varphi \mapsto \operatorname{Tr}(C \cdot(X \cdot(\partial \varphi) \cdot X+X \varphi))=\operatorname{Tr}(C \cdot(X \cdot \partial(X \varphi))-X \varphi) .
$$

The matrix coefficient expansions from Propositions 25, 26 and 27 have much larger regions of convergence than stated, the proofs remain the same. Since we use these expansions so often, we provide more precise statements.

Proposition 112. We have the following matrix coefficient expansion

$$
k_{0}(Z-W)=\frac{1}{N(Z-W)}=N(W)^{-1} \cdot \sum_{l, m, n} t_{n \underline{m}}^{l}(Z) \cdot t_{m \underline{n}}^{l}\left(W^{-1}\right),
$$


which converges uniformly on compact subsets in the region $\left\{(Z, W) \in \mathbb{H}_{\mathbb{C}} \times \mathbb{H}_{\mathbb{C}}^{\times} ; Z W^{-1} \in \mathbb{D}^{+}\right\}$. The sum is taken first over all $m, n=-l,-l+1, \ldots, l$, then over $l=0, \frac{1}{2}, 1, \frac{3}{2}, \ldots$

Proposition 113. We have the following matrix coefficient expansions

$$
k(Z-W)=\frac{(Z-W)^{-1}}{N(Z-W)}=\frac{1}{N(Z)} \sum_{l, m, n}\left(\begin{array}{c}
\left(l-m+\frac{1}{2}\right) t_{n+\frac{m+\frac{1}{2}}{l}}^{l}(W) \\
\left(l+m+\frac{1}{2}\right) t_{n \underline{n-\frac{1}{2}}}^{l}{ }^{\underline{m-1}}
\end{array}\right) \cdot\left(t_{m_{\underline{n-\frac{1}{2}}}^{l+\frac{1}{2}}}\left(Z^{-1}\right), t_{m \underline{m+\frac{1}{2}}}^{l+\frac{1}{2}}\left(Z^{-1}\right)\right),
$$

which converges uniformly on compact subsets in the region $\left\{(Z, W) \in \mathbb{H}_{\mathbb{C}}^{\times} \times \mathbb{H}_{\mathbb{C}} ; W Z^{-1} \in \mathbb{D}^{+}\right\}$. The sum is taken first over all $m=-l-\frac{1}{2},-l+\frac{3}{2}, \ldots, l+\frac{1}{2}$ and $n=-l,-l+1, \ldots, l$, then over $l=0, \frac{1}{2}, 1, \frac{3}{2}, \ldots$ Similarly,

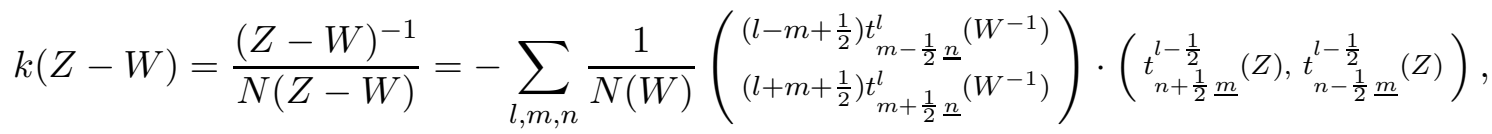

which converges uniformly on compact subsets in the region $\left\{(Z, W) \in \mathbb{H}_{\mathbb{C}} \times \mathbb{H}_{\mathbb{C}}^{\times} ; Z W^{-1} \in \mathbb{D}^{+}\right\}$. The sum is taken first over all $m=-l+\frac{1}{2},-l+\frac{3}{2}, \ldots, l-\frac{1}{2}$ and $n=-l,-l+1, \ldots, l$, then over $l=\frac{1}{2}, 1, \frac{3}{2}, 2, \ldots$.

Proposition 114. We have the following matrix coefficient expansions

$$
\frac{1}{N(Z-W)^{2}}=\sum_{k, l, m, n}(2 l+1) N(Z)^{k} \cdot t_{n \underline{m}}^{l}(Z) \cdot N(W)^{-k-2} \cdot t_{m \underline{n}}^{l}\left(W^{-1}\right),
$$

which converges uniformly on compact subsets in the region $\left\{(Z, W) \in \mathbb{H}_{\mathbb{C}} \times \mathbb{H}_{\mathbb{C}}^{\times} ; Z W^{-1} \in \mathbb{D}^{+}\right\}$. The sum is taken first over all $m, n=-l,-l+1, \ldots, l$, then over $k=0,1,2,3, \ldots$ and $l=$ $0, \frac{1}{2}, 1, \frac{3}{2}, \ldots$.

The representation $\left(\rho_{2}, \mathcal{W}^{+}\right)$introduced at the beginning of Subsection 4.2 is not irreducible. In fact, it is easy to see from Subsection 5.1 that $\left(\rho_{2}, \mathcal{W}^{+}\right)$has two irreducible components: $\left(\rho_{2}, \mathcal{Q}^{+}\right)$and $\left(\rho, H^{+}\right)$.

There are several sign errors in Subsection 4.3. In particular, $\tilde{A}$ should be defined as

$$
\tilde{A}=-A_{0} \tilde{e}_{0}+A_{1} e_{1}+A_{2} e_{2}+A_{3} e_{3}=-\tilde{e}_{0} A_{0}+\vec{A}
$$

(negative of the original $\tilde{A}$ ). Either way, the main conclusion still holds. Namely, that $\mathrm{Mx} \tilde{A}=0$ if and only if the Maxwell equations (expressed as equation (56) in [FL1]) are satisfied.

The main purpose of Subsection 5.1 was to describe the decomposition of the tensor product representation $\left(\pi_{l}^{0}, \mathcal{H}^{+}\right) \otimes\left(\pi_{r}^{0}, \mathcal{H}^{+}\right)$of $\mathfrak{g l}\left(2, \mathbb{H}_{\mathbb{C}}\right)$ into irreducible components due to [JV2. Unfortunately, the representations $\left(\rho_{n}, \mathscr{T}_{n}^{+}\right)$of $\mathfrak{g l}\left(2, \mathbb{H}_{\mathbb{C}}\right)$ are not irreducible for $n \geq 2$. Indeed, we saw in Section 5 that $\left(\rho_{2}, \mathscr{K}_{2}^{+}\right)=\left(\rho_{2}, \mathcal{W}^{+}\right)$is not irreducible. Thus, Theorem 82 in [FL1] can be corrected as

Theorem 115. The image of the intertwining map $M_{n}$ from Theorem 85 in [FL1] is an irreducible subrepresentation of $\left(\rho_{n}, \Psi_{n}^{+}\right), n=1,2,3, \ldots$

Let us denote this image by $\left(\mathcal{K}_{n}^{+}\right)_{\text {irr }}$. The irreducible representations $\left(\rho_{n},\left(\mathscr{W}_{n}^{+}\right)_{\text {irr }}\right), n=$ $1,2,3, \ldots$, of $\mathfrak{s l}\left(2, \mathbb{H}_{\mathbb{C}}\right)$ are pairwise non-isomorphic and possess inner products which make them unitary representations of the real form $\mathfrak{s u}(2,2)$ of $\mathfrak{s l}\left(2, \mathbb{H}_{\mathbb{C}}\right)$. follows

When $n=1$, we have $\left(\mathscr{K}_{1}^{+}\right)_{\text {irr }}=\mathscr{K}_{1}^{+}=\mathscr{K}^{+}$. Then equation (61) in [FL1] should read as

$$
\left(\pi_{l}^{0}, \mathcal{H}^{+}\right) \otimes\left(\pi_{r}^{0}, \mathcal{H}^{+}\right) \simeq \bigoplus_{n=1}^{\infty}\left(\rho_{n},\left(\mathscr{K}_{n}^{+}\right)_{i r r}\right)
$$


This decomposition is obtained by treating $\mathcal{H}^{+} \otimes \mathcal{H}^{+}$as functions of two variables $Z, Z^{\prime} \in \mathbb{H}_{\mathbb{C}}$ and filtering them by the degree of vanishing on the diagonal $\mathbb{H}_{\mathbb{C}} \subset \mathbb{H}_{\mathbb{C}} \times \mathbb{H}_{\mathbb{C}}$. Then

$$
\begin{gathered}
\left(\rho_{1}, \mathcal{K}^{+}\right) \text {generated by } 1 \otimes 1, \\
\left(\rho_{n},\left(\mathcal{K}_{n}^{+}\right)_{i r r}\right) \text { generated by }\left(z_{i j}-z_{i j}^{\prime}\right)^{n-1}, \quad n \geq 2 .
\end{gathered}
$$

Subsection 5.3 was written so it could be later used to give a proof of the "magic identities" for the conformal four-point integrals described by the box diagrams. Magic identities are proved in [L2] using different methods.

\subsection{Comments about [FL3]}

In the expression above Theorem 15

$$
\left(\left(I_{R}^{+-}+I_{R}^{-+}\right) N(W)^{-1}\right)\left(Z_{1}, Z_{2}\right)=-\frac{1}{N\left(Z_{2}\right)} \cdot \begin{cases}\frac{\log \lambda_{2}-\log \lambda_{1}}{\lambda_{2}-\lambda_{1}} & \text { if } \lambda_{1} \neq \lambda_{2} \\ \lambda^{-1} & \text { if } \lambda_{1}=\lambda_{2}=\lambda\end{cases}
$$

$\log$ denotes the branch of logarithm with a cut along the positive real axis and $\lambda \neq 1$. Thus when we let $Z_{1}, Z_{2} \rightarrow Z$ we need the eigenvalues $\lambda_{1}, \lambda_{2}$ of $Z_{1} Z_{2}^{-1}$ to stay on the same side of the cut:

$$
\lim _{\substack{Z_{1}, Z_{2} \rightarrow Z, N\left(Z_{1}-Z_{2}\right) \neq 0 \\ \operatorname{sign}\left(\operatorname{Im} \lambda_{1}\right)=\operatorname{sign}\left(\operatorname{Im} \lambda_{2}\right)}}\left(\left(I_{R}^{+-}+I_{R}^{-+}\right) N(W)^{-1}\right)\left(Z_{1}, Z_{2}\right)=-N(Z)^{-1}, \quad Z \in U(2)_{R} .
$$

Recall that $\widetilde{\mathcal{H} \otimes \mathcal{H}}$ denotes the space of holomorphic $\mathbb{C}$-valued functions in two variables $Z_{1}, Z_{2} \in$ $\mathbb{H}_{\mathbb{C}}$ (possibly with singularities) that are harmonic in each variable separately. Then Theorem 15 should be restated as

Theorem 116. The $\mathfrak{g l}\left(2, \mathbb{H}_{\mathbb{C}}\right)$-equivariant map

$$
f \mapsto\left(\left(I_{R}^{+-}+I_{R}^{-+}\right) f\right)\left(Z_{1}, Z_{2}\right) \quad \in \widetilde{\mathcal{H} \otimes \mathcal{H}}, \quad f \in \mathscr{W},
$$

where $Z_{1}, Z_{2} \in U(2)_{R}, N\left(Z_{1}-Z_{2}\right) \neq 0$, is well-defined and annihilates $\mathscr{T}^{-} \oplus \mathscr{T}^{+}$.

Moreover, we have a well defined operator $\mathrm{P}^{0}$ on $\mathscr{T}$

$$
f \mapsto\left(\mathrm{P}^{0} f\right)(Z)=\lim _{\substack{Z_{1}, Z_{2} \rightarrow Z, N\left(Z_{1}-Z_{2}\right) \neq 0 \\ \operatorname{sign}\left(\operatorname{Im} \lambda_{1}\right)=\operatorname{sign}\left(\operatorname{Im} \lambda_{2}\right)}}-\left(\left(I_{R}^{+-}+I_{R}^{-+}\right) f\right)\left(Z_{1}, Z_{2}\right), \quad Z \in U(2)_{R},
$$

which annihilates $\mathscr{T}^{-} \oplus \mathscr{W}^{+}$and is the identity mapping on $\mathscr{T}^{0}$.

Furthermore, the projector $\mathrm{P}^{0}$ on $\mathscr{T}$ can be computed as follows:

$$
\begin{aligned}
\left(\mathrm{P}^{0} f\right)(Z)=\frac{1}{2 \pi^{3} i} \lim _{\theta \rightarrow 0} \lim _{s \rightarrow 1} & \left(\int_{W \in U(2)_{R}} \frac{f(W) d V}{N\left(W-s e^{i \theta} Z\right) \cdot N\left(W-s^{-1} e^{-i \theta} Z\right)}\right. \\
& \left.+\int_{W \in U(2)_{R}} \frac{f(W) d V}{N\left(W-s^{-1} e^{i \theta} Z\right) \cdot N\left(W-s e^{-i \theta} Z\right)}\right), \quad Z \in U(2)_{R} .
\end{aligned}
$$

The fact that $\mathrm{P}^{0}$ is a projector onto $\mathscr{K}^{0}$ may be interpreted as

$$
M \circ\left(\left(I_{R}^{+-}+I_{R}^{-+}\right) f\right)=f \quad \text { if } f \in \mathscr{K}^{0} .
$$

Theorem 22 should be restated in a similar manner. In particular, the operator $\mathrm{P}_{\mathbb{M}}^{0}$ on $\rho_{1}(\mathscr{W})$ should be defined as

$$
\left(\mathrm{P}_{\mathbb{M}}^{0} f\right)(Z)=\lim _{\substack{Z_{1}, Z_{2} \rightarrow Z, N\left(Z_{1}-Z_{2}\right) \neq 0 \\ \operatorname{sign}\left(\operatorname{Im} \lambda_{1}\right)=\operatorname{sign}\left(\operatorname{Im} \lambda_{2}\right)}}-\left(\left(I_{\mathbb{M}}^{+-}+I_{\mathbb{M}}^{-+}\right) f\right)\left(Z_{1}, Z_{2}\right), \quad Z \in \mathbb{M},
$$

where $\lambda_{1}$ and $\lambda_{2}$ denote the eigenvalues of $\left(Z_{1}+1\right)\left(Z_{1}-1\right)^{-1}\left(Z_{2}-1\right)\left(Z_{2}+1\right)^{-1}$. 


\section{References}

[BBBV] S. Badger, N. Bjerrum-Bohr, P. Vanhove, Simplicity in the structure of QED and gravity amplitudes, JHEP 02 (2009) 038.

[BCFW] R. Britto, F. Cachazo, B. Feng, E. Witten, Direct Proof of the Tree-Level Scattering Amplitude Recursion Relation in Yang-Mills Theory, Phys. Rev. Lett. 94 (18), 181602 (2005).

[ES] M. Eastwood, M. Singer, A conformally invariant Maxwell gauge, Phys. Lett. A 107 (1985), 73-74.

[Er] A. Erdélyi et al, Higher Transcendental Functions. Based, in part, on notes left by Harry Bateman, Vol. I, McGraw-Hill, New York-Toronto-London, 1953.

[FL1] I. Frenkel, M. Libine, Quaternionic analysis, representation theory and physics, Advances in Math 218 (2008), 1806-1877; also arXiv:0711.2699.

[FL2] I. Frenkel, M. Libine, Split quaternionic analysis and the separation of the series for $S L(2, \mathbb{R})$ and $S L(2, \mathbb{C}) / S L(2, \mathbb{R})$, Advances in Math 228 (2011), 678-763; also arXiv:1009.2532.

[FL3] I. Frenkel, M. Libine, Anti de Sitter deformation of quaternionic analysis and the second-order pole, IMRN, 2015 (2015), 4840-4900; also arXiv:1404.7098.

[FL4] I. Frenkel, M. Libine, n-regular functions in quaternionic analysis, submitted.

[JV1] H. P. Jakobsen, M. Vergne, Wave and Dirac operators, and representations of the conformal group, J. Functional Analysis 24 (1977), no. 1, 52-106.

[JV2] H. P. Jakobsen, M. Vergne, Restrictions and expansions of holomorphic representations, J. Funct. Anal. 34 (1979), no. 1, 29-53.

[K] B. Keller, A-infinity algebras, modules and functor categories in Trends in Representation Theory of Algebras and Related topics, 67-93, Contemp. Math. 406, Amer. Math. Soc., Providence, RI, 2006.

[Le] S. T. Lee, On some degenerate principal series representations of $U(n, n)$, J. Funct. Anal. 126 (1994), 305-366.

[L1] M. Libine, The two-loop ladder diagram and representations of $U(2,2)$, Jour. of Lie Theory 27 (2017), 771-800; also arXiv:1309.5665.

[L2] M. Libine, The conformal four-point integrals, magic identities and representations of $U(2,2)$, Advances in Math 301 (2016), 289-321; also arXiv:1407.2507.

[P] S. Paneitz, Analysis in space-time bundles, III. Higher spin bundles, J. Functional Analysis 54 (1983), 18-112.

[V] N. Ja. Vilenkin, Special functions and the theory of group representations, translated from the Russian by V. N. Singh, Translations of Mathematical Monographs, Vol. 22 American Mathematical Society, Providence, RI 1968.

[Z] D. Zagier, The Dilogarithm Function. In: P. Cartier, P. Moussa, B. Julia, P. Vanhove (eds) Frontiers in Number Theory, Physics, and Geometry II. Springer, Berlin, Heidelberg 2007. 
Department of Mathematics, Yale University, P.O. Box 208283, New Haven, CT 06520-8283 Department of Mathematics, Indiana University, Rawles Hall, 831 East 3rd St, Bloomington, IN 47405 